\title{
Patterns of student learning in medical education : a Sri Lankan study in a traditional curriculum
}

Citation for published version (APA):

Marambe, K. N. (2007). Patterns of student learning in medical education : a Sri Lankan study in a traditional curriculum. [Doctoral Thesis, Maastricht University]. Datawyse / Universitaire Pers Maastricht. https://doi.org/10.26481/dis.20070620km

Document status and date:

Published: 01/01/2007

DOI:

10.26481/dis.20070620km

Document Version:

Publisher's PDF, also known as Version of record

\section{Please check the document version of this publication:}

- A submitted manuscript is the version of the article upon submission and before peer-review. There can be important differences between the submitted version and the official published version of record.

People interested in the research are advised to contact the author for the final version of the publication, or visit the DOI to the publisher's website.

- The final author version and the galley proof are versions of the publication after peer review.

- The final published version features the final layout of the paper including the volume, issue and page numbers.

Link to publication

\footnotetext{
General rights rights.

- You may freely distribute the URL identifying the publication in the public portal. please follow below link for the End User Agreement:

www.umlib.nl/taverne-license

Take down policy

If you believe that this document breaches copyright please contact us at:

repository@maastrichtuniversity.nl

providing details and we will investigate your claim.
}

Copyright and moral rights for the publications made accessible in the public portal are retained by the authors and/or other copyright owners and it is a condition of accessing publications that users recognise and abide by the legal requirements associated with these

- Users may download and print one copy of any publication from the public portal for the purpose of private study or research.

- You may not further distribute the material or use it for any profit-making activity or commercial gain

If the publication is distributed under the terms of Article $25 \mathrm{fa}$ of the Dutch Copyright Act, indicated by the "Taverne" license above, 


\title{
Patterns of Student Learning in Medical Education - A Sri Lankan study in a Traditional Curriculum
}

\author{
PROEFSCHRIFT
}

ter verkrijging van de graad van doctor aan de Universiteit Maastricht, op gezag van de

Rector Magnificus, Prof. mr. G.P.M.F. Mols,

volgens het besluit van het College van Decanen,

in het openbaar te verdedigen

op woensdag 20 juni 2007 om 14.00 uur

door

Kosala Nirmalani Marambe

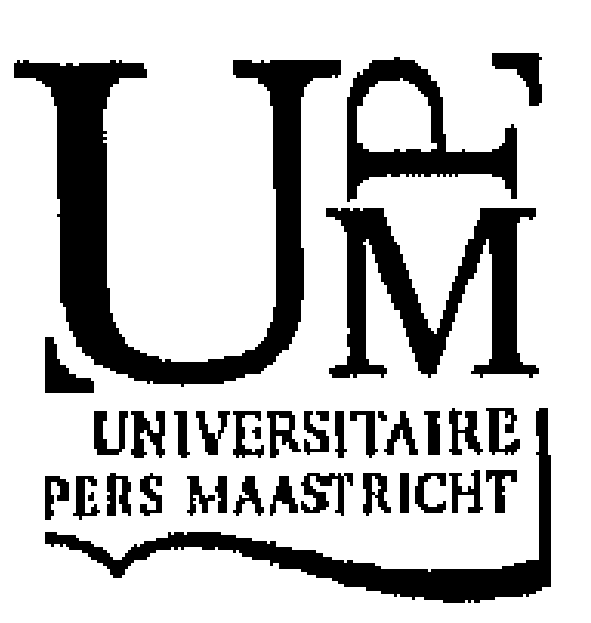




\section{Promotores:}

Prof. dr. J.D.H.M. Vermunt (Universiteit Utrecht)

Prof. dr. H.P.A. Boshuizen (Open Universiteit Nederland)

Beoordelingscommissie:

Prof. dr. C.P.M. van der Vleuten (voorzitter)

Dr. J. van Dalen

Prof. dr. A.E.M.G. Minnaert (Rijksuniversiteit Groningen)

Dr. H. Roebertsen

Prof. dr. M. Valcke (Universiteit Gent)

ISBN 978-90-5278-632-2

Datawyse, Universitaire Pers Maastricht 


\section{Acknowledgements}

The research in this dissertation aims to describe the learning patterns of Sri Lankan medical students studying in a traditional curriculum. The completion of my dissertation was made possible through the contribution of a number of people. I wish to express my sincere gratitude to those who contributed their advice, guidance and support throughout every stage of writing this dissertation.

First of all it gives me great pleasure to express ny heartfelt gratitude to my promotores, Prof. H.P.A. Boshuizen, Professor of Education and Educational Technology, Open University, the Netherlands and Prof. J.D. Vermunt, Professor of Teaching and Teacher Education, Utrecht University, the Netherlands for their inspiring and meticulous guidance, constant encouragement, constructive criticism, scholarly suggestions and benevolent and generous help rendered for the successful completion of this thesis.

Books can also be an important source of inspiration. In this respect, I would like to mention the inspiration derived from "The Experience of Learning - Implications for teaching and studying in higher education" edited by F. Marton, D. Hounsell \& N. Entwistle, which motivated me to explore the learning approaches of Sri Lankan medical students.

Furthermore, I am grateful to Dr. N.D. Kasturiaratchtchi, former Director and Dr. T.N.C. Athuraliya, present Director of the Medical Education Unit (MEU) for their encouragement and unstinted support throughout the research project. May I take the privilege of extending my sincere thanks to the staff officer's of the MEU, Mr. J.C. Udurawana and Mr. U.S.K. Ekanayake, for their timely help rendered to complete my research work. I also wish to express my sincere thanks to Mr. D.L. Wimalasena and Mr. S. Vipulasiri of the MEU for their valuable cooperation shown during my work.

I owe my sincere thanks to all the Deans of the Faculty of Medicine throughout the period of my study for their generous assistance and encouragement. A special word of thanks is extended to Dr. P.T. Jayawickramarajah of the World Health Organization for advising me to pursue my higher studies at Maastricht University, the Netherlands. I am also thankful to Dr. S. Samita, Senior lecturer in Biometry, Faculty of Agriculture, University of Peradeniya, for offering his expertise and clarifying issues related to data analysis whenever requested.

On a personal note, 1 extend my deep sense of gratitude to my husband Buddhi Marambe and my son Yoshan Marambe, who were a great source of continuous encouragement. Their incredible sacrifice, inexhaustible love, constant moral support, helped me to overcome the challenge of completing this thesis project. I am also grateful to my parents and my brother for sharing joys and frustrations throughout this period of study. I also owe my heartfelt thanks to my close associates for their constant encouragement and moral support.

A special word of appreciation and thanks is extended to the medical and dental students who participated in the different stages of the research project for their excellent cooperation. It is my duty to acknowledge the financial assistance obtained in the form of "World Health Organization Fellowships for Staff Development", which enabled me to initiate the thesis project and develop my skills during the process of investigation.

Last but not least, may I extend my sincere thanks to everyone who offered me kind and massive encouragement in the course of the research project. 


\section{Table of Contents}

1. General introduction 1

Objectives of curriculum reforms in higher education 1

Teaching and learning 1

Background and aim of the project 3

Objectives of the overall project 3

An overview of the chapters 4

2. Sri Lanka - Background to the country, its people and medical education 6

$\begin{array}{ll}\text { The country and its people } & 6\end{array}$

The educational system in Sri Lanka $\quad 7$

Current situation and formal education system in Sri Lanka 7

Evolution of general education $\quad 8$

$\begin{array}{ll}\text { Collegiate level of formal education } & 10\end{array}$

History of university education in Sri Lanka 11

Funding and admission $\quad 11$

University administration 13

Higher professional education $\quad 13$

$\begin{array}{ll}\text { Medical education in Sri Lanka } & 13\end{array}$

History of undergraduate medical education in Sri Lanka 13

Intake of students and admission procedures to medical schools $\quad 15$

$\begin{array}{ll}\text { Curricular } & 16\end{array}$

$\begin{array}{ll}\text { Staff } & 18\end{array}$

$\begin{array}{ll}\text { The accreditation process } & 19\end{array}$

The issues related to undergraduate medical education in Sri Lanka
affecting the quality of teaching

Medical education at Faculty of Medicine of the University of Peradeniya 23

The curriculum at the Faculty of Medicine of the University of Peradeniya

The existing curriculum at Faculty of Medicine, University of Peradeniya reviewed using the SPICES model 24

3. Student learning in higher education 27

$\begin{array}{ll}\text { Introduction } & 27\end{array}$

Learning as a complex phenomenon $\quad 27$

Conceptions of learning and knowledge $\quad 28$

$\begin{array}{ll}\text { Conceptualising student learning } & 29\end{array}$

Learning activities, approaches to learning, and learning strategies 29

Conceptions of learning and learning orientations 31

Cognitive styles, learning styles and learning patterns $\quad 32$

Research approaches to the study of student learning 33

$\begin{array}{ll}\text { A shift in research paradigm } & 33\end{array}$

$\begin{array}{ll}\text { Phenomenography } & 34\end{array}$

Study strategies inventories $\quad 34$

Research findings on student learning 38

Consistency and variability in students' learning strategy use $\quad 38$

Dissonance in student learning patterns $\quad 38$

Developments in student learning patterns over years of schooling $\quad 39$ 
Personal influences on student learning $\quad 40$

Relations between student learning and academic performance 41

The context of student learning and approaches to teaching 42

The context of learning and its influence on student learning 42

Student learning in medical education $\quad 44$

Student learning in higher education in Sri Lanka 46

Teacher centered vs student centered academic practice $\quad 46$

Process oriented teaching $\quad 47$

Problem based learning $\quad 48$

Implications for curriculum design $\quad 49$

The role of culture in shaping educational and learning practices $\quad 50$

Dimensions of a national culture $\quad 50$

Cross-cultural research on student learning $\quad 51$

Conclusions

4. Introduction to the empirical studies 55

Background $\quad 55$

The Sri Lankan study $\quad 55$

A conceptual model underlying this dissertation $\quad 56$

$\begin{array}{ll}\text { Research context } & 58\end{array}$

Empirical study 1 described in chapter $5 \quad 59$

Study 2 described in chapter $6 \quad 60$

Study 3 described in chapter $7 \quad 60$

Study 4 described in chapter $8 \quad 61$

5. Beliefs and views of Sri Lankan new entrant medical students on learning 62

Introduction $\quad 62$

Individual differences in learning $\quad 62$

Qualitative research methods $\quad 63$

Role of interviews and scope in the present study 63

Research questions $\quad 64$

Method

Participants $\quad 64$

Interview schedule $\quad 65$

$\begin{array}{ll}\text { Procedure } & 65\end{array}$

Data analysis $\quad 65$

Results

Attributes of an ideal student $\quad 68$

Factors facilitating learning $\quad 69$

$\begin{array}{ll}\text { Study strategies } & 70\end{array}$

Conceptions of learning and knowledge $\quad 73$

$\begin{array}{ll}\text { Attributions of academic success and failure } & 74\end{array}$

Student expectations about learning in higher education $\quad 75$

Student expectations of the role of a university teacher $\quad 76$

Study motives $\quad 77$

Associations between learning conceptions, learning strategies, regulation strategies and expected learning in the university $\quad 80$

Discussion and conclusions 
6. Development of the adapted version of the inventory of learning styles: the Adyayana Rata Prakasha Malawa

Introduction

Selection of a measuring tool

The inventory of Learning styles (ILS) and its development

Development of the Sri Lankan version of the Inventory of

Study 2 Learning Styles (ILS): the Adyayana Rata Prakasha Malawa (ARPM)

Introduction

Method $\quad 93$

Results $\quad 94$

$\begin{array}{ll}\text { Study } 3 & 95\end{array}$

Development of ARPM $2 \quad 95$

$\begin{array}{ll}\text { Method } & 96\end{array}$

$\begin{array}{lr}\text { Results } & 97\end{array}$

$\begin{array}{ll}\text { Conclusions and discussion } & 104\end{array}$

What is the reliability of the ARPM-1 scales? To what extent can the internal consistencies of scales be increased?

Which learning styles of Sri Lankan students can be identified by ARPM-2?

What is the relationship between students' ARPM scale scores and personal factors such as age, gender, attempt at entry and average amount of independent study time per day?

What are the relationships between students' ARPM scale scores and contextual variables such as level of parental education/ occupation, district from which the student has secured entry to medical faculty?

What is the association between ARPM scale scores and academic performance?

Is there a relationship between the ARPM scale scores and students' locus of control?

What is the academic locus of control of medical students? 107

7. A cross-cultural comparison of student learning patterns 109

Introduction

Cultural differences in approaches to learning $\quad 109$

$\begin{array}{ll}\text { Culture specific behaviours } & 110\end{array}$

$\begin{array}{ll}\text { Family of instruments } & 110\end{array}$

Internal structure of learning styles among first year

Dutch University students using the ILS

Internal structure of learning styles among first year Indonesian

University students using the ICB

Internal structure of Learning styles of Sri Lankan

medical students identified with the ARPM 111

$\begin{array}{ll}\text { Goals of the present study } & 112\end{array}$

Methodology

Data analyses $\quad 114$

Results 
Conclusions and discussion

Do Sri Lankan students differ from Dutch and Indonesian students in terms of the reported use of study strategies, orientations of learning and conceptions of learning?

Similarities and differences in the internal structure observed in the two Asian and the Western cultures

Theoretically incongruent learning patterns among the three cultural groups

Learning environment as an explanatory factor

Asian culture as an explanatory factor

8. Trends in the development of self-regulated learning among undergraduate medical students within the context of a traditional medical curriculum

Introduction

Aims and objectives of the present study

124

Method

Research questions

Study 3: Cross-sectional study

Study 4: Longitudinal study

Participants

Materials

Procedure

Study 5

Data analysis

Students

Materials

Procedure

Data analyses

Study 4

Changes in the learning strategies, learning orientations, and conceptions of learning among first year students and third MBBS students

Third year MBBS students

Comparison of the factor structures of the first year batch of students at time 1 and time 2

Comparison of the factor structures of the 1996/1997 batch of students at time 1 and time 2

Intercorrelations of ARPM scale scores between time 1 and time 2 for the two batches of medical students

Study 5

Internal consistencies of the Course Experience

Questionnaire (CEQ) scales

Conclusions and discussion

Do learning strategies, conceptions of learning and learning orientations differ between three groups of students who differ in the study phase they are in, i.e. new entrants, third year and fourth year medical students? 
Do reported learning strategies, conceptions of learning and learning orientations differ within two groups of students at two moments in time with an interval of 30 weeks and 50 weeks?

How does the factor structure underlying the ILS change during two stages of the MBBS course?

How consistent or variable are students' learning strategies, conceptions of learning and learning orientations?

How do first and fourth year students perceive their learning environments?

9. General conclusions and discussion

Introduction

Main findings of the overall project: summary and discussion

Sri Lankan medical students' ways of learning: the qualitative study 146

From the ILS to ARPM: reliability and validity

Learning patterns of Sri Lankan medical students

Relations between learning patterns and personal and contextual factors and academic performance

The Asian paradox

Developmental trends in learning patterns

Students' perceptions of the existing learning environment 155

Implications for educational practice

Directions for change

$\begin{array}{ll}\text { General indications for future research } & 160\end{array}$

10. References

Summary 


\section{General introduction}

\section{Objectives of curriculum reforms in higher education}

Learning in higher education can be viewed as developing a way of thinking and acting that is characteristic of an expert community. Learning thus consists of two important elements: (1) the knowledge that represents phenomena in the subject domain, and (2) the thinking activities that are used to interpret situations in that domain and to act in these situations. Similarly with regard to professional education, Lindblom-Ylänne (1999) stated, "development of expertise involves the process of developing domain specific competence in addition to epistemological development and a shift towards more selfregulated and meaning-directed learning" (p. 21). With the overwhelming mass of biomedical information and the ever-increasing development of scientific discovery, the need for medical students to become effective, self-directed learners who are able to continue learning after the formal training has ended, becomes indisputable (Davis \& Harden, 1999). Thus, the emphasis nowadays is on both what students should learn (the content) as on how students should learn (the process).

The objective of present day curriculum reforms is basically to improve the quality of learning processes that students realize. Studies have shown that the quality of knowledge gained by active knowledge construction is better (more accessible, coherent and usable) than knowledge acquired by passive means (Vermunt \& Verschaffel, 2000). Therefore, higher education institutions nowadays pay greater attention to the development of active learning among undergraduate students than before.

As Vermunt (1996) observed, "... for many senior jobs, it is seemingly ever less important what academic subject someone has qualified in (e.g. 'an academic education'), if only the candidate has these thinking skills (...): somebody with analytic skills, someone who knows how to readily discern relevant from irrelevant information, has the capability to make knowledge usable, can motivate oneself and others, can independently plan and monitor work activities, and so on" (p. 26-27). Since aspects of life are changing at a rapid speed, it has become impossible to teach people everything that is known in their subject domain during their relatively short stay at the university. Furthermore, contemporary societies have become learning societies necessitating lifelong learning for everybody (Van Hout-Wolters, Simons, \& Volet, 2000). Today's society needs people who are able to learn. This has motivated the educational institutions to change their teaching approaches and shift the emphasis from imparting factual knowledge, which might even become redundant by the time these students graduate, to developing learning and thinking skills. Thus, the society expects the higher education institutes to promote active learning by providing opportunities for undergraduates to practice how to learn and think. Against this background, learning to learn and hence teaching students how to learn and think independently to become the masters of their own advancement have become major goals of education (Boekaerts, 1997; Hounsell, 1997; Vermunt \& Verschaffel, 2000).

\section{Teaching and learning}

Trigwell, Prosser, \& Waterhouse (1999) reported that qualitatively different approaches to teaching are associated with qualitatively different approaches to learning. Today theories of teaching are grounded in constructivist theories of learning that assume learning to 
be a process of active knowledge construction. Hence, teaching is viewed as stimulating learners to employ suitable thinking activities to construct, change and utilize their knowledge. Recently, Trigwell and Prosser (2004) were able to show that there are systematic relations between the ways teachers' teach and the quality of their students' learning.

Knowledge is situated, being in part a product of the activity, context and culture in which it is developed and used. This situational nature of learning has been emphasized by many researchers (Brown, Collins, \& Duguid, 1989; Ramsden, 1997; Vermetten, Vermunt, \& Lodewijks, 1999a). On the same line, Genn (2001) argued that the climate of an educational institution is a powerful determinant of learning behaviour of both its students and teachers. However, conventional schools seem to pay less attention to the influence of school environment and culture on what is learned.

Despite intellectual homogeneity, a number of environmental and personality varjables affect an individual's behaviour, resulting in great heterogeneity among a student population within any discipline. Unfortunately, students are often taught as if individual differences do not exist. One of the important findings that has emerged from educational research is that students approach their studies in different ways. Marton and Säljö (1997) pointed out that motivation and intention drive the learning process, including the level of cognitive processing. In the literature on effective learning two qualitatively different approaches to learning have been described, i.e. a surface and a deep approach (Marton \& Säljö, 1997). Surface learners focus on memory strategies, are extrinsically motivated and reach a low level of understanding of the subject matter. On the other hand, students using the deep approach actively try to understand the meaning of the subject matter by working out relationships between concepts, relating new material to previously known information and adopting a critical attitude to information.

Coming to the issue of quality of learning in different learning contexts, the meaning oriented learning approach is considered to be the most desirable for undergraduate students. Learning processes in which pure surface or passive approaches are used are assumed to represent lower quality. Empirical studies have shown that institutions, which have incorporated certain educational innovations, record a substantial increase in deep processing strategies and self-regulation among its students (Trigwell \& Prosser, 2004; Vermunt \& Minnaert, 2003). Underlying the concepts of "learning approach", "learning style" and "learning pattern" lays the integration of components of learning such as intention, motivation and cognition. Furthermore, there is a growing body of evidence that combinations of the preferred learning style and the context produce characteristic learning approaches that are recognizable in all groups of students, including medical students (Lindblom-Ylänne, 1999; Vermetten, 1999).

Recently, Boyle, Duffy, and Dunleavy (2003) pointed out that the links between learning components and how these may change in different educational environments, demand further investigation. Hence, knowledge about students" "learning patterns", "styles" or "approaches" within a given learning environment has potential usefulness for teachers in the curriculum development process and for adjusting their way of teaching to maximize learning achievement.

In actual practice didactic teaching methods are employed fairly extensively within the Sri Lankan medical school context. This is sometimes due to reasons beyond the control of the faculty (e.g. restrictions such as a low staff student ratio and scarcity of resources). Didactic teaching methods are based on external regulation of students' learning processes. In this respect, Vermunt (1998) pointed out that the regulation power of didac- 
tic measures such as learning objectives, questions, directions for studying and assignments, is minimal with regard to the direct activation of relating and structuring, critical and concrete processing strategies. Thus, classical instructional design theories, with their high emphasis on external regulation of learning processes by instructional measures, seem to work only to a limited extent or for a limited group of students. An extra matter of concern is whether this high degree of external control leads to reproduction-directed learning (see Biggs' (1996) and Shuell's (1996) discussion of this problem). Previous research findings on student learning patterns repeatedly questioned the advantages of exerting a high degree of external control over learning processes by instructional agents.

\section{Background and aim of the project}

At present there is a trend of reforming undergraduate medical curricula all over the world. The Sri Lankan higher education system too, is discussing ways of reforming its curricula as well as carrying out staff development activities to promote desirable learning strategies among its participants (UGC, 2000). As is the case with most Asian countries, we too are somewhat cynical about change. Within this atmosphere convincing a majority of members of a medical school that such changes are beneficial and that spending time on curriculum reforms is a worthwhile exercise, is a very difficult task. Therefore, the present study was conducted to broaden the understanding of the learning process among medical students, thus paving way for more informed decision-making during the curriculum development process. It is expected that these studies, by showing relations between the institutional level contextual factors, student approaches to learning and learning outcomes, may through the weight of evidence give change agents the support they need to convince their academic and administrative colleagues.

\section{Objectives of the overall project}

This project had eight major objectives:

1. To identify and describe the learning strategies, conceptions of learning, academic locus of control, learning motives, attributes of an "ideal student", and views on teaching and learning in higher education of Sri Lankan undergraduate medical students at the commencement of their studies.

2. To develop a valid and reliable diagnostic instrument, the Adyayana Rata Prakasha Malawa (ARPM), that could be used to get an overview of the study habits and learning styles or patterns of Sri Lankan undergraduate medical students in the Faculty of Medicine, University of Peradeniya.

3. To investigate relations between ARPM scale scores and academic performance, level of parental education and occupation, district from which the student had secured entry to medical school, average reported study time, gender, attempt in which university selection is secured, and dimensions of academic locus of control.

4. To explore the attribution pattern (academic locus of control) of Sti Lankan medical students at different stages of the MBBS course.

5. To identify the similarities and differences in the reporting of learning strategies, conceptions and orientations among first year university students within a cross- cultural perspective. 
6. To identify consistency and variability of the use of learning strategies, learning orientations and conceptions of learning across time and contexis among medical students.

7. To explore the developmental trends in respect of the four leaming components (the use of processing strategies, regulation strategies, reporting of conceptions of leaming and learning orientations), among Sri Lankan medical students within the context of a traditional curriculum.

8. To identify possible contextual factors that may promote the observed changes in learning patterns.

\section{An overview of the chapters}

Chapter 2 describes the educational system in Sri Lanka with special emphasis on medical education; a developmental history with special emphasis on medical education; the present status of medical education; the strengths and weaknesses etc., thereby orientating the reader to make his/her inferences from the studies described in the next chapters.

Chapter 3 describes the theoretical background of the study, reflecting the main theoretical concepts, and their relations as applicable to this study. It discusses the theoretical notions in educational psychology such as: constructivism, teaching and learning, the role of the leaming context, research methodologies employed in exploring study strategies and learning conceptions, problem based learning and process oriented instruction, and the role of the national culture in education.

Chapter 4 is an introduction to the empirical studies. It describes the research project giving the background to the study, the research context and the conceptual model underlying the thesis.

Chapter 5 is a qualitative chapter, exploring the learning strategies during senior secondary education as well as beliefs and views in respect of the learning process of medical students at the commencement of the course. It comprises an analysis of the results of semi-structured interviews conducted with new entrant students. It gives an account of some of the learning conceptions, study habits, attributions of successes and failures among group of new entrant, medical students', and aspects such as motives behind selecting to study Medicine and their view of an "ideal student", and teaching learning processes in the university. Thus, chapter 5 is mainly directed to meeting objective one.

Chapter 6 is directed at objectives 2,3 and 4 . It reports the adaptation of the Inven tory of Learning Styles (ILS), developed by Vermunt $(1996,1998)$, to create the Sri Lankan version of the ILS, the Adyayana Rata Prakasha Malawa (ARPM). The chapter furthermore reports the reliabilities of scales and the factor structures identified anong Sri Lankan medical students. The associations between variables such as academic performance in medical school examinations, study time, district of entry, parental education and occupation were also investigated. In addition, the attributions (locus of control) of medical students at three stages of the medical course are explored in this chapter.

In chapter 7, objective 5 is investigated in detail. An attempt is made to compare the results of three studies that have used either the ILS or one of its adapted native versions, among first year higher education students from varying disciplines. Results are discussed within a cross cultural framework.

Chapter 8 is aimed at meeting objectives 6,7 , and 8 . In this chapter, developments in medical students" use of learning strategies, conceptions of learning and learning orientations within a discipline based traditional curriculum are discussed, in both a cross sectional and a longitudinal design. The chapter further points out some of the possible con- 
textual factors that may contribute to observed trends in the reported use of learning strategies and conceptions of learning.

Finally, in chapter 9 the overall conclusions that emerge from these studies are discussed in a broader perspective. Implications for medical educational practice, desirable changes in current instruction and suggestions for further research complete this chapter. 


\section{Sri Lanka - Background to the country, its people and medical education}

\section{The country and its people}

Sri Lanka, formerly known as Ceylon, is an island located $880 \mathrm{~km}$ north of the equator, off the southern tip of India. It measures $435 \mathrm{~km}$ at its longest and $225 \mathrm{~km}$ at its broadest. The total land area is approximately 62,705 square kilometres, one fifth of which consists of natural forests while another two fifths is cultivated land. The surface area has a welldefined central mountainous region with peaks as high as 2,524 meters, which is surrounded by plains stretching down to sea. The island being close to the equator has a tropical climate, with a mean temperature of 26-28 degrees centigrade in the low country and 14-24 degrees centigrade in the central mountainous region.

The present population of 19.6 million (Central Bank, 2005) is multi-ethnic and the Sinhalese majority, who are mainly Buddhists, constitute $74 \%$ of the population. Tamils who are mainly Hindus, constitute $18.2 \%$ of the population, while Moors, who follow Islam, constitute $7 \%$ and the descendants of the former colonists (Dutch, Portuguese), called Burghers, Malay and others constitute the remaining $0.8 \%$. About $5 \%$ of the total population is Christian.

The country has clear evidence of flourishing civilization over 2000 years, the remains of ancient art and sculpture and the great reservoirs and canal system, which flourished the agro-economic life style bears testimony to this effect. The recorded history of Sri Lanka goes back to the advent of Indo-Aryans from India in $543 \mathrm{BC}$. Arahath Mahinda introduced Buddhism from India in the third century $\mathrm{BC}$ and it became the established religion and the focus of nationalism. Until the sixteenth century $A D$, the country had an independent, monarchical system of governance. From 1505, the Portuguese, Dutch and British dominated the maritime areas successively. In 1815, the British became the first European power to rule the entire island and thus had a considerable impact on the socio-economic life of the country. With the dawn of the $20^{\text {th }}$ century a nationalist movement comprising Buddhists and Hindus was started demanding self-government. As a result in 1931 the recommendations of the Donoughmore Commission were implemented and a system of semi-autonomous government was formed. Finally, Sri Lanka regained political independence in 1948 and became a Democratic Socialist Republic in 1972. It is unfortunate that during the last two decades the country had to face the consequences of an on going war steered by the Liberation Tigers of Tamil Elam demanding self-government in the northern and the eastern provinces of the country.

Sri Lanka, although a developing South Asian nation, has many achievements to its credit. The trend in the overall literacy (defined as the percentage of the population aged 10 years and over who are able to read and write at least one language) has been upward and now stands at $92.5 \%$ (Central Bank, 2005). This is much higher than most countries in South Asia region and the Developing countries (the south Asian average is $49 \%$ and the Developing countries average is $72 \%$ ). It is heartening to note that higher aspirations of parents for the education of their offspring both boys and girls have largely eliminated gender disparities in school enrolment at national level. During the long period of its evolution since the 1940s, the free education system both in schools and the universities has produced scholars of high calibre. Universities have indeed been in the forefront of the 
process of human capital formation for the country's socio-economic and human development.

The average life expectancy has shown an upward trend over the years. The life expectancy as it now stands is 71.7 years for males and 76.4 years for females (Central Bank, 2005), a figure, which is comparable to the developed countries in the world. This combined with a progressive decline both in the population growth rate (1.1) and the fertility rate $(2.3 \%)$ has resulted in a gradual narrowing of the base of the population pyramid. In 2005 the proportion of the population below 15 years was $26 \%$.

The major exports of the country include garments, gems, tea, rubber, and coconut. The average per capita income is around US $\$ 1190$ (Central Bank, 2005). About 33\% of the labour force is in agriculture, $26 \%$ in industry and $41 \%$ in the services. Since 1977 the change of government has brought about a market-oriented, export-led liberalized economy, which has as its objective the reduction and ultimately the elimination of subsidies and a shift away from maintaining an expensive social welfare system.

The country has a democratic parliamentary system. The parliament, in which the people's sovereignty and legislative powers are vested, exercises authority through a cabinet of ministers presided over by an executive president. The President and Members of the Parliament are elected directly by the people. The Ministry of Higher Education and Information Technology is responsible for making policy decisions regarding university education. Hence medical education is a responsibility of the above ministry while the Ministry of Health is directly responsible for delivery of health care through the public sector and it also oversees health care delivery in the private sector.

For administrative purposes the country is divided into eight provinces viz. Western, Southern, Northeastern, Central, Northcentral, Northwestern, Sabaragamuwa and Uva, 25 districts, and 318 divisional secretary areas. The general trend in the country is towards decentralization of administrative responsibilities and authority to the provincial level.

\section{The educational system in Sri Lanka}

\section{Current situation and formal education system in Sri Lanka}

Formal education in Sri Lanka begins at the age of five years and can be categorized into four stages (see Table 2.1). They are primary, junior secondary, senior secondary and collegiate level. Primary education refers to a child's education during the first five years in school and is an important phase in human development. The majority of primary school population will be in the age range of five to nine years. The grade span is from 1 to 5 .

Junior secondary education spans from grade 6 to 9 while senior secondary education includes grade 10-11. Learners follow the following subjects; first language, mathematics, Englisir, science, social studies, religion, aesthetics and health and physical education. Students after eleven years of education sit the first public examination, which is the General Certificate of Education - Ordinary Level (GCE O/L). Those who qualify for collegiate level education study for another two years in a selected stream, i.e. biological science, mathematics, commerce, languages (and choose three subjects relevant for the given stream) and sit the university entrance examination. The latter examination is called the General Certificate of Education - Advanced Level (GCE A/L). Based on the performance at the latter examination students are selected for different university undergraduate courses. Table 2.1 indicates the formal education system, the average age at which each level is commenced up to the collegiate level. 
Chapter 2

With regard to formal education there are four types of schools satisfying the educational needs of the Sri Lankan population, namely government schools, semi-government schools, private schools and international schools. In the international schools the medium of instruction is English and the students sit for foreign examinations. Very recently a number of private schools have been established which coach students for local examinations in the English medium.

Table 2.1. Formal Education System in Sri Lanka as at present

\begin{tabular}{|c|c|c|c|c|c|c|c|c|c|c|c|c|c|c|}
\hline Age (Yrs) & 5 & 6 & 7 & 8 & 9 & 10 & 11 & 12 & 13 & 14 & 15 & 16 & 17 & 18 \\
\hline Grade & 1 & 2 & 3 & 4 & 5 & 6 & 7 & 8 & 9 & 10 & 11 & 12 & 13 & \\
\hline $\begin{array}{l}\text { Type and } \\
\text { duration of } \\
\text { Education }\end{array}$ & \multicolumn{5}{|c|}{$\begin{array}{l}\text { Primary Education } \\
\text { (5 years) }\end{array}$} & \multicolumn{4}{|c|}{$\begin{array}{c}\text { Junior Secondary } \\
\text { Education } \\
\text { (4 years) }\end{array}$} & \multicolumn{2}{|c|}{$\begin{array}{l}\text { Senior Sec- } \\
\text { ondary Edu- } \\
\text { cation ( } \\
\text { years) } \\
\text { GCE O/L }\end{array}$} & \multicolumn{3}{|c|}{$\begin{array}{c}\text { Collegiate level } \\
\text { Education (2 } \\
\text { years) } \\
\text { G.C.E. A/L }\end{array}$} \\
\hline
\end{tabular}

The total expenditure on education as a percent of GDP (Gross Domestic Product) was 2.4 in 2002, 2.2 in 2003, 2.1 in 2004 and 2.7 in 2005 (Central Bank, 2005). This is much lower than the allocation made in the immediate post independent era (which was around $5 \%$ of GDP). Statistics reveal that this decreasing trend has commenced since 1970. It is also noted that the expenditure on public education as a percentage of GDP falls below the South Asian average, which is reported to be 3.4 (Earthtrends, 2004). The most significant development in the field of education in the latter part of the last decade was the island wide implementation of education reforms in 1999. Several foreign funded projects are in progress to improve general education in the country, i.e. the World Bank funded teacher-training project, the ADB funded secondary education development Project, etc. (Perera, 2003). Objectives of these reforms were to minimize the gap between educational attainment and the labour market requirements by improving the quality of education, ensuring equity in resource allocation, and providing adequate input and infrastructure facilities. The reforms envisage a system of education, which would empower students with necessary knowledge, skills and attitudes to make them productive citizens of Sri Lanka and to create a future generation with desired values, compassion and care towards fellow citizens. Furthermore, regulations to make education compulsory from grade 1 to 9 have been enacted in 1998 as a major objective of the reform programme in order to realize the UNESCO goal of education for all.

\section{Evolution of general education}

Prior to the foreign invasions in the $16^{\text {th }}$ century the Buddhist monks handled education. However, during the colonial period there was a two-tier system of education, i.e. fee levying English schools, and non-fee levying Sinhala and Tamil schools. The latter dealt with primary education and the former dealt with secondary education (Central Bank, 1998). Moreover, Sinhala and Tamil schools taught little beyond basic English literacy skills (De Silva \& De Silva, 1990). Very briefly the key feature of general education prior to the changes in educational policy in the late 1940's (during the latter part of the colonial period) was that secondary education was provided almost exclusively in schools that taught in English medium (De Silva \& De Silva, 1990). Secondary education in English 
led students to the legal and medical professions or to other respectable positions in the government service. Thus the most glaring inequality was between opportunities available for those who studied through the English language and the students who learnt through Sinhala or Tamil. The then education system created a privileged minority and a disadvantaged majority based on the language of instruction (De Silva \& De Silva, 1990). However, the conflicts and resentments that the above situation aroused influenced the education policy in the post-independent era. Thus extending equal opportunities to all with respect to education became a national goal in the pre-independent era.

The Education ordinance No. 31 of 1939, which was enacted after a long deliberation, was aimed at extending educational opportunities and that remains the basic law of Education in Sri Lanka. Sri Lanka is proud of having been able to provide free education to every citizen of the country through legislature long before the Universal Declaration on Human Rights by the United Nations.

A major breakthrough in the education system was the introduction of free education for all students up to the university level. This decision was made in accordance with the recommendations made by the C.W. W. Kannangara report in 1944 (Central Bank, 1998). In 1943 a special committee on education worked on the premise that every individual must have equal opportunities, so that provided the person has the necessary innate ability, s/he can lift himself/herself in the social and political life of the nation. Subsequently, in the latter part of 1950's the medium of instruction in secondary education and in some fields of university education was changed to Swabhasha (Sinhala or Tamil) by a legislative enactment. Furthermore, several reforms have taken place with respect to primary and secondary education during the past three decades improving accessibility (age limit for school entry, free provisioning of school text books, school uniforms and midday meal programme are some of the changes made) and improving the curriculum (changing teaching learning methods, structure of the public examinations, number and types of subjects offered to students) (Central Bank, 1998). The net enrolment in primary education is reported to be $97.9 \%$ in the year 2005 (Central Bank, 2005). Unfortunately, policies and practices in education in Sri Lanka have been subject to changes in the political party in power. As a result some of the original, beneficial policy decisions, which would have improved the quality of education were neglected thus leading to the current state of affairs with increasing regional disparities in the quality of general education.

Over three decades since 1970 the country was faced with problems of unemployment, youth unrest and ethnic conflict, which aggravated poverty. The question whether education had fully played its role in imparting knowledge, skills and attitudes for all to face the challenges in the millennium, was given serious thought. Personality development characterized by respect and tolerance of others, creativity, cliscipline and team spirit were also found to be deficient among youth. Thus, restructuring and reforms in the education system was identified as a priority. Accordingly, the National Education Commission was established in 1991 to study the problem and make recommendations for reforms. Subsequently a presidential task force was appointed to implement the above recommendations in 1996. The year 1997 was declared the Year of Education Reforms and reforms in all aspects of education gained momentum (Ministry of Human Resource Development, Education and Cultural Affairs, 2004).

Since 1999 the general education reform programme has been implemented island wide (Central Bank, 1999). During the years, the curricula and methodology of teaching were completely revised for several grades in the primary and secondary stages, while a compulsory general English paper was introduced at the GCE A/L (Central Bank, 1999; 
2003). Furthermore, new national colleges of education and teacher training centres were established for the purpose of pre-service and in-service teacher training; it is expected to have a substantial impact on improving the quality of learning.

Measures have been taken to enhance computer literacy among school children. Furthermore, arrangements were made to establish sophisticated computer education centres on a regional basis, with the aim of promoting computer based education (Central Bank, 2005). Educationists state that the educational reforms, among other things are expected to enhance learning to learn, analytical and critical thinking and problem solving skills and foster desirable interpersonal skills, so that the future citizens would be in a better position to compete for jobs in the global market and would also develop a good team spirit which would also pave the way for much sought after national unity.

Accordingly, new syllabi were developed, textbooks re-written and teacher guides prepared in line with the reform objectives. i.e. to improve the quality of learning, development of competencies in contrast to pure content of subject matter, promote self education and ability to solve problems that may arise under unforeseen circumstances and enhance interpersonal skills, and communication skills. Students are also made aware of the importance of environmental conservation for human existence. Teachers are trained to take up the challenge of guiding the students to be independent learners. It is stated that, a comprehensive teacher re-training programme was implemented via the teacher centres that are established, while the maximum number of students per class is being reduced to 35, enabling more student- teacher interactions (Central Bank, 2003; Pieris, 2004). In keeping with the present trends in education teaching learning activities have been modified, use of information technology for teaching and learning purposes in the classroom is encouraged, more individual and group assignments have been incorporated into the school curriculum. A shift of emphasis from summative evaluation to formative evaluation is also envisaged. Teachers are also instructed to monitor the mastery in essential learning competencies of their students identified for each key stage thus, a move towards criterion based assessment rather than norm referenced assessment is obvious in the new system. The education reforms have also addressed the improvements in school administration, mechanism for monitoring learning progress in the schools and curriculum review and reform activities (Pieris, 2004).

\section{Collegiate level of formal education}

The number of subjects offered to a student at the collegiate level was reduced from four to three subjects since 1998. For example, those who are interested in pursuing a career in biosciences are required to study chemistry, physics and biology. Subject content and teaching learning methods have also been modified with the introduction of project work and independent assignments. Having identified the need to improve the English language skills of the students, a compulsory new course on "General English" was introduced for GCE A/L students in 1999. The GCE A/L examination tests the knowledge of all these subjects. In addition, a common general paper, designed to test students' awareness of current affairs, reasoning ability, problem solving ability and communication skills, was introduced as a part of the GCE A/L examination for those who seek admission to the universities (Ministry of Education, 2004). However, English marks are not added to the aggregate marks for university admission.

With regard to the education system, Sri Lanka has been having a high enrolment in primary and secondary education. Of the schooling population in the country, about $98 \%$ of the age group five to ten years old has enrolled in primary education, and $35 \%$ of the 
age group of seventeen and eighteen years has enrolled in senior secondary education (Central Bank, 2005). However, the total participation in tertiary education (that is the total enrolment in the thirteen national universities, open university and other higher educational institutes) has been far from compatible with the high enrolment in primary and secondary education. It is estimated to be less than $4 \%$ of the age cohort $20-24$, in comparison with more than $25 \%$ in UK, France and Australia. In most of the Newly Industrialized Countries (NICs) it is in the range of 6-8\% (Gunawardena, 1999). Thus by international comparison, the Sri Lankan intake into tertiary education is low as a proportion of the population in the relevant age group, creating a large excess demand for university education that cannot be fulfilled in the country.

\section{History of university education in Sri Lanka}

The history of academic education in Sri Lanka is marked by two important dates: 1870 and 1942. In 1870 Ceylon Medical School was established. In 1880 the school was raised to the status of college permitting it to award the Licentiate in Medicine and Surgery (LMS) and in 1889 the General Medical Council of the United Kingdom recognized the College when holders of its license became eligible to practice in Great Britain (Varagunam, 1996). At this time the country was a British colony, and hence educational practices in the institution followed the British pattern.

In 1942 the University of Ceylon was established. It emanated from the continuous efforts of a group of public-spirited citizens who formed the Ceylon University Association in the early years of the twentieth century. Their aim was the establishment of a university in Sri Lanka. As a result of their persistent efforts the Ceylon University College, affiliated to the University of London was founded in 1921. It prepared students for the external examinations conducted by the University of London. The founders considered it a preliminary step in the ultimate creation of a degree awarding university, which happened in 1942 (Lakshman, 1998). With the establishment of the University of Ceylon by statute, tertiary education was formalized in the country (Central Bank, 1998).

With the establishment of two new universities in 2005, the number of state universities in the country (excluding the Open University) has risen to fifteen (Central Bank, 2005). In the year 2004, apart from the enrolment in the Open University, 13,396 new students entered these universities while the number has increased to 14,250 in 2005 (Central Bank, 2005). This accounts for fourteen and thirteen percent of the students who satisfied the minimum requirements for university entrance respectively and less than four per cent of those who enter primary education at the age of five years (UGC, 2004a). In addition to the national universities, there are five higher educational institutes. The Open University of Sri Lanka caters to a student population of approximately 24,450 while several private sector institutes have also commenced higher education programmes (mostly in the fields of information technology, accountancy, law and business management) in collaboration with foreign universities (Central Bank, 2003).

\section{Funding and admission}

According to Lakshman (1998) the fundamental premises on which the system of university education in Sri Lanka has developed over the years are as follows:

Funding The university education should be a state sector activity. This public sector monopoly of university education has, however, been eroded over the recent past in an indirect way. Some private educational institutions have come up to train Sri Lankan stu- 
dents for degrees offered by foreign universities, colleges, etc. However, no institution exists in the private sector, which awards its own degrees (i.e. no fee-levying fullyfledged universities exist in Sri Lanka), in spite of extensive liberalization of many other activity areas in the country. It is noted that there is no provision to set up private universities in Sri Lanka under the existing Universities act. This situation has deprived those citizens who can afford to pursue higher education in a fee-levying institute (unlike in the neighbouring countries where there are numerous private universities). However, the current trend is that those who can afford international university education send their children abroad for higher education since the Sri Lankan system, which is described below, is extremely competitive. As a result Sri Lanka's annual national expenditure bill on overseas education is estimated to be substantial. The university education up to the Bachelor's degree level is free of tuition fees and those qualifying to enter universities for such degree programmes are provided with scholarships and bursaries. The registration, examination and such other fees charged and, when hostel facilities are provided, the rental charges for such facilities are kept at highly subsidized levels.

Admission Admission to universities is centrally controlled on the basis of a set of transparent and objective criteria, in which "merit" as determined by the results of an examination, weighted according to differential education facilities available in different districts, plays the major role. In the early stages (1942 onwards), students for university admission were selected entirely on merit. The GCE A/L, which is conducted by the Ministry of Education, is the selection test for university entrance in Sri Lanka. Due to a number of reasons, equity considerations have been added to merit in the selection of students for university admission and thus, university admission is made on dual criteria viz (a) all island merit, and (b) merit on district basis, resulting in a quota system.

In the case of courses other than arts, up to $40 \%$ of available places are filled in order of the marks compiled on an all island merit basis. Fifty five per cent of the available places in each course of study are allocated to the 25 administrative districts proportionately, based on the ratio of the population of each district to the total population of the country. The remaining $5 \%$ is allocated to the 13 educationally disadvantaged districts proportionately, based on the ratio of the population of each such district to the total population of the 13 districts. According to Lakshman (1998) following are some of the reasons for this differential allocation of available places in the national universities:

1. Wide discrepancies in the facilities available in the schools, which train students for the university entrance examination.

2. Difficulties of maintaining uniform standards across students sitting this examination in different language media.

3. Concerns of the politicians representing under-privileged regions. University admission criteria have undergone several changes over the last three decades, before arriving at the current system.

At the early stages of evolution of local university education i.e. after the establishment of the university of Ceylon in 1942 by the Ceylon University ordinance no. 20 of 1942, this central examination for university admission was conducted by the single university of that time. Subsequently, its place was given to the General Certificate of Education (GCE). Now the department of examinations under the Ministry of Education conducts the GCE A/L examination. This examination taken by school children at the end of thirteen years of schooling has become the only avenue for entry into university undergradu- 
ate study programmes as an internal student. Each internal student has three attempts to qualify for selection to an undergraduate course in a state university at present.

\section{University administration}

The University Grants Commission (UGC) under the Ministry of Higher Education, functions as the apex body in the university system. UGC which was established in 1979, functions as the central admission agency, determines admissions to universities on the basis of criteria decided in consultation with the government, ensures standards in the appointment of academic and non-academic staff to universities by laying down schemes of recruitment, fosters interaction and achieves a measure of coordination among universities through the medium of standing committees or inter-university foruns, which have been established for all major academic disciplines with representatives from each university (UGC, 2003).

Uniformity must be ensured among different universities through central control in respect of salaries and other benefits offered to the academic and other personnel, their recruitment and promotion schemes, subsidies offered to students and such other matters. Though, theoretically, the degrees offered by different universities in any given discipline are considered equal for official purposes, it is known that they differ in quality due to resource constraints (Lakshman, 1998).

\section{Higher professional education}

The Faculties of Medicine, Engineering, Dentistry, Architecture, Veterinary Sciences, Law and Management Studies offer professional courses. The medium of instruction in all the professional faculties has continued to be English and the education imparted by those faculties has retained an element of elitism. Undergraduate places in these faculties have generally been in strong demand and there has been a tendency that those students who score higher aggregates at the Advanced Level examination, who are more hard working and who are more well to do opt for courses in the above faculties. In general, education is regarded by the Sri Lankan society as a path of upward mobility. Individuals who excelled in studies could overcome social barriers (De Silva \& De Silva, 1990). Furthermore, professionals could obtain respectable jobs as well as form alliances with the elite family circles. All of these factors have made academic professional education in Sri Lanka highly respectful and thus, very competitive.

\section{Medical education in Sri Lanka}

\section{History of undergraduate medical education in Sri Lanka}

The history of medical education dates back to 1870 . It was not until the $19^{\text {th }}$ and $20^{\text {th }}$ centuries that medical education in the allopathic system began to be introduced into the South-East Asian region (World Health Organization, 1996). Thus, the Ceylon Medical College that was established in Colombo by the British rulers is known to be the third medical school in the region. At this time the country was a colony of Great Britain, and hence educational practices in all educational institutions in the country, including those in the medical school, followed the British pattern.

The decision to set up the Ceylon Medical School was part of an overall strategy for improving the general health of the population and for stopping the deaths due to malaria and other tropical diseases. It is for this reason that in the early years of its existence the 
national body responsible for health care, the Ministry of Health, was responsible for the administration of the Medical School. Later in 1942, when tertiary education was formalized in the country the medical school became part of the government funded university system and remained so until present (Varagunam, 1996). Hence the Colombo Medical College located in the western province became the first Faculty of Medicine, of the University of Colombo.

Later in 1961, the second medical school in Sri Lanka was established in the Central province. This is the Faculty of Medicine, University of Peradeniya (FMUP), where the studies reported in this thesis were conducted. Subsequently, Faculties of Medicine were established in the Southern and the Northern provinces around the same time in 1979. Over the years, there had been a rapid increase in the country's population from around 6 million in the 1940s to the current figure of over 19 million, leading to a greater demand for health care and for health personnel.

In keeping with the increased demand in the period 1979-1996, the universities have recorded an expansion in both student numbers and number of institutions. As a result the number of students admitted for a year to a particular faculty has doubled. Most recently established medical schools are the Faculty of Health Sciences of the University of Sri Jayawardenapura and the Faculty of Medicine of the University of Kelaniya, both of them being located in the western province. The latter was actually a private medical college that was started in 1985. It did not survive long due to much public criticism and opposition and was taken over by the government system. At present there are no privately funded medical schools in the island. Six of the publicly funded universities have faculties of medicine and the total annual intake is around 905 students (UGC, 2004a). The Ministry of Health so far had been the principal employer of medical graduates.

At the inception the local medical school curriculum was described as a "carbon copy of the British medical curriculum". Major changes in medical education first began in the region with the introduction of preventive and social medicine in the 1960s followed by the community medicine movement that was promoted in 1970s (World Health Organization, 1996). Along with the rest of the world Sri Lankan medical schools incorporated these developments. From that time on the undergraduate medical training that took place only within the four walls of the tertiary care hospital was extended to a field setting.

Introduction of educational science and technology into medical education had become one of the most vigorously pursued activities in the mid 1970s (World Health Organization, 1996). In keeping with the global trend the Medical Education Unit of the FMUP was established in 1972 to fulfil a regional and a national need. Thus, the FMUP had been a pioneer in initiating staff training in the Faculties of Medicine and Allied Health Sciences. As a result more than $80 \%$ of the teaching staff in the Sri Lankan medical schools at that time have been exposed to the principles and practices of educational science. Since then the unit has conducted several workshops with the participation of experts in the field and was successful in introducing some of the newer concepts, such as active learning, objective examinations, student research projects and learning assignments to the country. Thus, it has played a key role in developing a human resource pool, which is potentially capable of innovative thinking and teaching. Today almost all the medical schools have their own medical education units, which are engaged in curriculum review and staff development. 


\section{Intake of students and admission procedures to medical schools}

Since the medical schools in Sri Lanka are solely supported by public funds, financial difficulties resulted in increased government intervention in the planning and governance of the medical institutions, whose priorities are not always those of the government. The present trend is to increase the intake of students to each faculty rather than increasing the number of faculties. The total intake of medical students has been increased from 120 in 1961, when there was only one medical school, to 905 in 2003. Also the number of students admitted to the Peradeniya Medical faculty increased by about a hundred percent in the last one and a half decades. The batch presently admitted to the Peradeniya Medical faculty consisted of 178 students. As shown in Table 2.2, the Faculties of Medicine, Colombo and Peradeniya admit the highest numbers of students for a given batch.

Table 2.2. Intake of students to faculties of Medicine in the last six academic years (number per batch).

\begin{tabular}{|l|c|c|c|c|c|c|}
\hline $\begin{array}{l}\text { Admission } \\
\text { year }\end{array}$ & Colombo & Peradeniya & Sri Jayawardenapura & Kelaniya & Jaffina & Ruhuna \\
\hline $1998 / 99$ & 195 & 173 & 148 & 160 & 100 & 117 \\
\hline $1999 / 00$ & 192 & 175 & 150 & 156 & 93 & 130 \\
\hline $2000 / 01^{*}$ & 193 & 174 & 147 & 159 & 79 & 129 \\
\hline $2001 / 2002^{*}$ & 190 & 177 & 149 & 155 & 70 & 130 \\
\hline $2003 / 2004^{*}$ & 190 & 175 & 150 & 160 & 100 & 130 \\
\hline $2004 / 2005$ & 197 & 178 & 152 & 160 & 84 & 133 \\
\hline
\end{tabular}

Source: UGC $(2004 \mathrm{c}) *$ in these years double batches of stadents have been taken in.

The trend shown here has been broken several times. During the latter part of the $1980 \mathrm{~s}$ the universities have been closed for more than three years due to civil unrest in the country. This resulted in long waiting lists for accepted students. To clear that backlog the faculties admitted two batches of students per year until 2004. For a university like Peradeniya running two batches means that 350 students are admitted in two groups several months apart. Needless to say that this has led to this has led to logistical problems, cut down vacations, etc.

The admission procedure of to state universities is highly competitive. In general due to the limited number of places available in the state universities less than $15 \%$ of those who are eligible are admitted (UGC, 2004b). With the introduction of the educational reforms from the year 1999 a couple of features of the admission procedures changed. While the number of compulsory subjects for collegiate level have been reduced to three (physics, chemistry and biology), students who sat for GCE A/L examination were also required to take a general knowledge paper as well; a pass for this component of the exam was needed for university admission. Another change was the introduction of the "Z-score" standardization system (based on the national mean and standard deviation) in university selection instead of the raw aggregate system adopted earlier. This has affected students' choice of subjects since it is no longer profitable to choose those areas where many students can easily score high marks. Presently the selection of students is done on the basis of the rank order on average $\mathrm{z}$-scores obtained by candidates (UGC, 2004b). The Ministry of Education and Higher Education and the university grants commission (UGC) regulate admissions based on the rank order of the average z-scores obtained at the GCE A/L exams and a district quota system (UGC, 2003). In order to be considered for entry to the medical faculty, the student should have passed in each of the three subjects, chemistry, physics, and biology; and should also have obtained marks of 
not less than $30 \%$ for the common general paper (UGC, 2003). Only those who obtain very high z-scores in a given district will be selected for medicine. This leads to different admission scores depending on the district the candidate resides. Minimum marks for selection to medicine in 2003/2004 ranged from 2.155 for the district of Colombo to 1.0910 (lowest cut-off mark) from the Northern Mannar district.

\section{Curricula}

Description of the existing curriculum The curriculum in most medical schools is similar, strictly discipline based and to some extent community oriented, except in the Faculty of Medicine University of Colombo, which has an innovative curriculum with considerable integration (Jayasinghe, 2002). Recently Faculty of Medicine, Kelaniya has also embarked on a system based integrated curriculum. Main thrusts of the new curriculum at the Faculty of Medicine University of Colombo are to integrate subjects (and departments), use diverse teaching methods and to place greater emphasis on in-course assessments (Jayasinghe, 2002).

The undergraduate curriculum in most of the medical faculties takes roughly the same duration and the same subjects are studied in an order very much similar to the British curriculum that was introduced decades ago. The structure of the curriculum in most medical schools still reflects the recommendations made by Flexner in 1900 (Jayasinghe, 2002). Initially for about 90 weeks the basic disciplines of anatomy, biochemistry and physiology are studied. On completion of these the students sit for an examination in these subjects (second MBBS examination). Passing this examination is a prerequisite for proceeding to the next phase of the curriculum (Varagunam, 1996). The second phase runs in parallel with clinical clerkships and consists of lectures and practicals in microbiology, parasitology, pharmacology, community medicine, pathology and forensic medicine. Students carry out their clerkships in the traditional disciplines of general medicine and surgery paediatrics and obstetrics and gynaecology as well as psychiatry and specialized clinical disciplines as ENT, ophthalmology, sexually transmitted diseases, orthopaedics, etc. In addition to the compulsory clinical appointments, some medical schools offer students the opportunity of elective clinical clerkships for a specified period. This second phase lasts for about 120 weeks and the students have to sit for the third MBBS part I and part II examinations respectively, towards the end of the third and fourth years of the course. In these examinations students are tested only in the para-clinical disciplines. The third phase of the curriculum, which lasts about 40 weeks, consists of clinical practice and lectures in medicine, surgery, paediatrics and obstetrics and gynaecology. On completion, students sit for the final MBBS examination that consists of written and practical assessments. The marks of the final examination are crucial to obtain good placements for internship training and for subsequent appointments in the Ministry of Health. Therefore the examination is fiercely competitive and causes considerable stress to the students concerned. The nominal duration of the curriculum is five years. However, the medical course may be lengthened, due to factors beyond the control of the faculty, such as student unrest, the political environment, staff agitation, etc.

Trends in curricular content There appears a trend within medical schools towards a systems approach whereby student's learning experiences are clustered around the physiological systems of the body. For example, students may be exposed to the anatomical, 
physiological and biochemical aspects of the nervous system concurrently, so that they may integrate their knowledge around the systems of the body.

Jayasinghe (2002) described three models of curriculum change, i.e. intradepartmental curriculum change, interdepartmental curriculum change where a number of departments jointly implement innovating teaching learning sessions, the third being introducing new structures outside the departmental structure such as modules or course units, which are coordinated by groups of staff members drawn from the departments (the structural changes or reforms). The latter requires major changes to the organizational structure of the medical faculty; it affects larger number of stakeholders and is often met with a very high degree of resistance.

In most medical schools new methods of teaching and learning have been incorporated into the teaching programs of individual departments. The Faculty of Medicine in Colombo has incorporated a problem-based learning approach in some of the innovative modules (Jayasinghe, 2002). However, the extent of changes varies from department to department. Departmental level changes are met with minimal resistance, as they require changes within the department only and a small group of academics is involved to implement such changes. These changes are common. The situation is not the same in the case of inter-departmental curriculum change and structural reforms in medical schools like introducing integrated (horizontal and vertical) teaching sessions (Jayasinghe, 2002). Given the limitations it is admirable that the Faculties of Medicine of Colombo and Kelaniya have been bold enough to embark on a systems based integrated curriculum. Secondly, a trend towards earlier exposure of students to the actual clinical problems is observed. For example, when discussing blood sugar metabolism in the first or second year a teacher will discuss the actual clinical problems that occur in patients with diabetes mellitus. Thirdly, introducing students to the community from the commencement of the course is seen in some of the medical schools (Varagunum, 1996). Fourthly, the introduction of a behavioural sciences stream to develop the communication skills, interpersonal skills and attitudes of medical students is becoming apparent. Finally, there is an emerging trend of including electives in the undergraduate curriculum in which students are given the chance (individually or in groups of two) to develop and conduct a research project or work in a specific area of their choice and submit a report on completion of the programme under an appointed supervisor (Jayasinghe, 2002).

Other trends in medical education had been the introduction of social and behavioural sciences into the undergraduate curriculum and greater emphasis on discussing ethical issues in health care. Increasing numbers of teachers express the view that medical school curricula place too much emphasis on memorization of facts and too little stress on self-directed study skills necessary for the practice of medicine in this millennium. Some of the established medical schools are making an effort to adopt new ways of organizing curricula, i.e. an organ-system-based model or a problem-based model, to improve the quality of learning. Fostering of teamwork among students, a trend in medical education in the region, has yet to become a prominent feature of educational programmes in some of the Sri Lankan medical schools.

Assessment A move towards more objective examinations is apparent in all the medical schools. In some disciplines the examinee is required to respond to fixed choice of answers (Multiple Choice Questions - MCQs). In the present context of very large batches of students, whose capacities to express themselves in English are not as great as they were in the past, MCQs have become important as an assessment tool (Varagunum, 
1996). There appears a trend towards the development of objective structured clinical and practical assessments and formal assessment of clinical appointments. Further a shift in emphasis from end-of-course assessments to in-course assessments is apparent in some departments. The situation at Peradeniya is that assessment is discipline based and emphasis is on end-of-course evaluations. The final MBBS examination is conducted separately by each faculty but always with examiners from the other faculties of medicine in Sri Lanka.

Staff

Faculty of Medicine There is a shortage of trained academic staff particularly in the pre-clinical and para-clinical departments. Medical graduates are not motivated to join these departments for a number of reasons. The newly graduated doctors prefer to work in the health care sector as they have a greater chance of engaging in private practice after working hours and to generate more income. We also witness a striking increase in the number of female lecturers in the medical faculties. In the meantime student intake has increased several fold resulting in a high student teacher ratio. However, there are substantial differences between the faculties. The Faculty of Medicine at Sri Jayawardenapura, located very close to Colombo, seems to be better off with a relatively low student teacher ratio. Staff-student ratios are again negatively influenced by the fact that at any particular time some of the permanent staff members are on study leave to pursue higher studies or on sabbatical leave. Faculties that are located outside the western province seem to be suffering most (Table 2.3). The Faculty of Medicine of the University of Jaffna, which is in the war torn area, is the worst affected.

Table 2.3. Permanent cadre of academic staff in the six medical schools

\begin{tabular}{|l|c|c|c|c|c|c|}
\hline Year & Colombo & Peradeniya & Sri Jayawardenapura & Kelaniya & Jaffna & Ruhuna \\
\hline 2001 & 114 & 95 & 78 & 79 & 12 & 77 \\
\hline 2002 & 120 & 92 & 90 & 84 & 15 & 74 \\
\hline 2003 & 124 & 96 & 102 & 82 & 13 & 78 \\
\hline 2004 & 119 & 98 & 98 & 84 & 21 & 74 \\
\hline
\end{tabular}

Source: UGC (2003); UGC (2004a)

Extended staff of the Faculty of Medicine From the inception of medical education in Sri Lanka, health ministry consultants have been assigned teaching responsibilities on a honorary capacity. Therefore, all consultants appointed to the teaching hospitals and some of the base hospitals take part in clinical training. Students commence their clinical rotations from the third year of study and the extended faculty conducts clinical training from then onwards.

Internship training The Medical Council grants provisional registration to all medical graduates, who have obtained the MBBS degree, while the Ministry of Health provides internship training of one year (houseman training). These graduates are registered in the medical council only on successful completion of this houseman year so that they are eligible to practice in the governmental or private sector. Usually this houseman training includes two six-months internships in any two of the clinical disciplines that constitute the final MBBS examination (either surgery and medicine or paediatrics, obstetrics and gynaecology). Although to-date the Ministry of Health had provided internship training and 
permanent employment to all the medical graduates, the situation is expected to change in the near future as the ministry has already indicated that it has a full cadre of doctors. Hence medicine, once considered to be a way of secured employment, also fears the absence of a assured job market like the other professional and non-professional courses.

\section{The accreditation process}

The Sri Lanka Medical Council (SLMC) is the accreditation body for all medical schools in Sri Lanka. The SLMC arose from its predecessor, the Ceylon Medical Council, by the 1987 ordinance that was enacted after the country became a republic. It consists of 21 members: five persons nominated by the Hon. Minister of Health, eight persons elected by different organizations in the health profession and a nominee from each medical faculty, a dental surgeon, and a person representing the assistant medical practitioners. The Sri Lanka Medical Council therefore consists of personnel from every sphere of the health sector. The functions of the SLMC are to license doctors to practice western medicine in Sri Lanka and to regulate standards of medical education, which is usually done through a process of recognition of new medical schools as producing graduates of a sufficient standard for temporary registration by the SLMC. Once the school is granted full recognition, there is no formal procedure for periodic re-assessment of the quality of medical education. However, it is anticipated that the SLMC and the medical faculties will embark on constant monitoring and thereby ensure the standards of medical training.

In the recent past the social accountability aspect of medical training has been of greater concern. Unlike in the other countries of the region, Sri Lanka is unique in the area of medical training as it is solely a public funded enterprise. During the long period of its evolution, the achievements of the system of university education have been substantial, although at present most commentators point to various weaknesses of the country's education system (Lakshman, 1998). These deteriorating standards have been attributed to various reasons, mostly being political (government policies) and administrative. For instance, a questionnaire survey conducted by Rajapakse (1996) among nonuniversity consultants in different hospitals of Sri Lanka under whom the trained graduates work, had revealed that a majority of the consultants are of the opinion that the medical education as well as ethical standards are deteriorating over the years. Furthermore, Sri Lankan expatriates who return to the country for short periods to work as visiting lecturers increasingly point out the deficiencies in the undergraduate training regarding instructional practices and problem-solving skills among medical students (T. De Silva, Kandy - personal communication to the Dean, Medicine, 2002).

The issues related to undergraduate medical education in Sri Lanka affecting the quality of teaching

Medium of instruction All professional courses in Sri Lanka are conducted in English. However, unlike in the post-colonial era, primary and secondary education has been conducted in "Swabhasha" (Sinhalese or Tamil languages) during the past three decades. Although, English is taught as a second language in schools up to grade 10 (GCE O/L), learning English has not been emphasized during advanced level studies. Since English language competence of a student is not an entrance criterion for admission to professional courses in Sri Lanka, this may have resulted in the selection of students without sufficient English language competencies. This change in the medium of instruction from 
Swabhasha to English is identified as a major constraint and is thought to be exerting a negative influence on the quality of the learning process of a university student.

In the latter part of the 1980 s English Language Teaching Units (ELTU) were established in all the medical faculties for the purpose of conducting an intensive English language course of three months duration for the new entrants. The ELTU staff considers it a difficult task to train a considerable number of these students within the given time as some of them are far behind the required standard (Ellawala, C., Fernando, C., \& Jayasekera, H., ELTU, University of Peradeniya - Personal communication to Curriculum Committee of the Faculty of Medicine, 2003). If indeed the English language communication skill of a considerable proportion of the undergraduate medical students is not up to the required level (De Silva, Babapulle, Marambe, \& Edussuriya, 2001), then this has implications for teaching and learning.

Selection criterion The selection procedure adopted by the University Grants Commission is also a matter of concern among faculty. Academics of the different faculties have frequently expressed their doubts about the appropriateness of the selection procedure, based on their experience that some of the new entrants, irrespective of their score in the GCE A/L examination system do not have the desired learning skills to pursue university education (Lakshman, 1998). These students do not have any or enough of the following: suitable critical thinking skills, the desire to learn new things, a well-developed reading habit, and/or communication skills. This criticism partly regards the examination concerned, but more importantly the teaching methods adopted by the schools to train students for that examination (Lakshman, 1998).

The learning environment and resources Statistics reveal that the total expenditure on education including universities has steadily decreased over the years. It had been $3 \%$ of the GDP in 1948 at the time of independence, as high as $5 \%$ in 1950 s when the expansion of educational institutions and quality of education was given due consideration. However, records indicate that the proportion has remained below 3 percent of the GDP over the last decade (Central Bank, 2005). The expenditure on public education as percentage of GDP in the South Asian region is around 3.4\%. Furthermore, total expenditure on universities has been between 0.4 to $0.5 \%$ of GNP over the last five years (UGC, 2004a).

The universities report that they have been well equipped up to the early 1970s. Most of the resource constraints have been imposed from the mid 1980s, and may be considered a reflection of the socioeconomic situation of the country that prevailed thereafter due to the ethnic conflict and the overwhelming defence expenditure, which amounted to $3-5 \%$ of the GDP.

Insufficiency of human and material resources, in view of the increased number of students, has changed the character of medical education in the country. The increased intakes into medical schools and the opening of medical schools within a short span of time without adequate planning have resulted in shortages of trained staff, larger classes, large number of students per teacher, greater reliance on lecturing as a teaching method (since it is a very economical method of transmitting knowledge) and allocation of large numbers of students per clinician during clinical clerkships. Some of the present tutorial classes have as many as 25-40 students. Steinert (1996) remarked that the optimum number of students in a small group is six to ten, though a group of twelve may also work. A 
group of twenty is not a small group; the number of students in a group has consequences on the discussion process.

Staff Shortage of trained staff in the medical faculties (pre-clinical and para-clinical departments are the worst off) has led to a high student teacher ratio $12: 1$. In the view of some educationists the country lacks a comprehensive plan for the development of human resources (Varagunam, 1996). As a result, existing teachers are overburdened with conducting lectures and student assessments. So much so the teachers frequently complain of having to devote extra time for teaching functions, service functions and peripheral faculty affairs. Compared to the situation in the past opportunities for the younger staff members for career advancement particularly in relation to some fields of study are very limited. In some areas of study local resource personnel are scarce. High student / teacher ratio (overall ratio in universities had been 10 in 1970, and increased to 12.6 in 2000) is identified as a constraint for curriculum reform activities as well (Central Bank, 1998; 2000). The poor remuneration package offered to academics seems to further reduce their commitment of time and energy to university work and is speculated to produce undesirable ripple effects in the system (Lakshman, 1998). Some of the consequences are lack of team spirit, poor coordination of multidisciplinary courses, lack of interest in systematic curriculum development, teachers resisting to change as it is additional work, etc. However, some teachers are commended for the hard work they put in, even under the limitations pointed out in this chapter.

Staff development in educational technology (teacher training) had been a neglected area for a considerable length of time, in the sense that there had been hardly any political commitment towards these activities. Although the Medical Education Unit was established with the above objective, the commitment of the policy makers towards its activities over the last decade is debatable. Lack of educational background knowledge might be the reason why most of the criticisms of staff in respect to educational reforms are based on anecdotal experiences; they often express ignorance of educational psychology and newer educational methods (Jayasinghe, 2002). Nevertheless, it is heartening to note that the aspect of staff development has gained momentum now under the directions of the university grants commission, as a priority area in the reform agenda and is expected to have a considerable impact on teaching and learning in medical schools (UGC, 2003). Furthermore, successful completion of a teacher-training course has been made mandatory to all newly recruited teachers, in the university system and it is required for tenure. Such regulations would invariably have an impact on the teaching leaning process.

Contribution of clinicians in the teaching hospitals (non-university teachers) towards the training of medical graduates is substantial. At present about 60 health ministry consultants are engaged in teaching the medical students in the respective clinical clerkships (Faculty of Medicine, 2000). However, the Ministries of Higher Education and Health are yet to launch systematic efforts to build team spirit among teachers of both Ministries. Development of a stronger relationship between the Ministry of Health and the Ministry of Higher Education will be beneficial. Providing training in educational technology to these health ministry consultants is an area that has not received much attention.

Administrative inefficiencies Delays and shortcomings in the administration procedures have led to a slowing down of the implementation of higher education reforms (Central Bank, 2000). The administrative and financial aspects of the faculty are regulated 
by the Universities Act No 16 of 1978 (UGC, 2000). Adherence to the stated outdated university act is also identified as a hindrance to academic development. According to this University Act the university senate is the supreme body for academic decisions. The act gives the decision making power at the local level to the faculty board comprising all academic staff (90 members), two student representatives, two directors of teaching hospitals, etc. The dean, who is elected for a three-year term has minimal statutory power to make policy changes, even on academic matters. Thus it can be argued that the faculty board with more than 90 members is not the forum for policy making. A new university act has been drafted to overcome some of the drawbacks in the university system and was discussed at different forums (Central Bank, 2003), however, it is yet to be enacted. The organizational structure of the universities with a strict hierarchy, rules and regulations is also felt to be a hindrance to educational innovations such as integration. Thus it is a big challenge for the established institutions to make radical changes to the curriculum. Although educational reforms in universities has been a subject of intense debate and discussion among the academia it is moving very slowly due to lack of a thorough action plan and commitment on the part of some staff members.

Resource allocation issues It is necessary to remind us of the fundamental premise on which the university education system is developed in Sri Lanka that it is solely a publicly funded enterprise. Therefore, the resource the state is able to provide to universities would depend on (a) the degree of prosperity and the rate of economic growth of the country, which eventually determine the level and expansion of government revenues, and (b) the structure of priorities in the allocation of available government revenues.

These have had significant implications for the quantity and quality of education imparted. It is a fact that the survival and growth of universities have come to depend primarily on resources, which the state can afford to allocate for them. Over the last fifteen years or so as well as in the present context, because of overwhelming defence expenditure, resource flow into the university sector is kept at mere survival level (Lakshman, 1998). Recent catastrophes of nature have only increased the pressure on the national budget. The proportion of GDP available for universities for their recurrent and capital needs may still be less than $0.5 \%$. As a result of resource constraints and inefficient utilization of available funds medical schools as a part of the university system, remain poorly equipped in terms of necessary buildings and teaching equipment.

The problems mentioned above are interconnected and constitute a vicious cycle of events. However, the future seems to be somewhat promising as the present governments expressed commitment to educational reforms. The current university education reforms focus primarily on expansion of university education, curriculum reform, quality assurance, administrative improvements, staff development, career guidance, counselling, and finance. The presidential task force that was appointed in 1997 to make recommendations on reforming the university education in the country has studied various aspects of the university system. Their recommendations cover key areas such as expansion of university education, curriculum revision, staff development, incorporate information technology in the teaching learning activities (UGC, 2000). In accordance with these, most universities have initiated action on curriculum reforms and staff development activities (UGC, 2004b). It is also anticipated that the government would continue to address the problem of decline in real terms of resources going into the university system in the context of a several- fold increase in the size of the university system. Staff development ac- 
tivities have gained momentum though not optimum, some faculties securing funds from donor agencies for the same.

In this background all universities have been requested to take action to implement the reform proposals as a matter of high priority. In 2004, universities were requested to conduct self-evaluations of their institutions. Furthermore, with the quality assurance process taking off the ground, individual faculties were also made to evaluate their academic programs, teachers and other staff and formulate their own reform agenda to upgrade the quality of the graduates thus, enabling them to compete in the local and international markets. Universities are encouraged to embark on outreach activities and income generation projects in order to sustain the quality of the academic programs offered, as the government funds are grossly inadequate to keep pace with the developments in the rest of the world.

\section{Medical education at Faculty of Medicine of the University of Peradeniya}

As stated earlier the FMUP was established as the second medical school in Sri Lanka to meet the growing need for medical professionals in the country. The first batch of students (95 in number) had been admitted in 1962. Initially this faculty was affiliated to the Faculty of Medicine University of Colombo. Thus the Dean of the Faculty of Medicine in Colombo functioned as the Dean of this new faculty, while the various departments were considered sub-departments of the ones in Colombo. It was only in 1967 that the faculty was converted to an independent medical faculty and later became the Faculty of Medicine of the University of Peradeniya (Faculty of Medicine, 2000). At present (2005), about 1100 students comprising six batches are undergoing training at this Medical Faculty. The undergraduate curriculum at the Peradeniya Medical Faculty occupies five years) and is arranged in trimesters (Faculty of Medicine, 2000).

The mission statement of the FMUP is: "The Medical Faculty is entrusted with the holistic education of health professionals of all kinds with ethical values and mutual respect, in an environment of excellence, where their education and training, the provision of preventive curative services to individuals and the community and research are seen to be part of a continuum. The foundations of and a desire for continuing education, and a spirit of enquiry and the recognition of responsibility to improve the health of the people, must be consequences of such education" (Faculty of Medicine, 2000, p. 1). Furthermore, FMUP in its institutional objectives states that student on completion of the training should among other things be capable of Life long learning (Faculty of Medicine, 2000).

\section{The Curriculum at Faculty of Medicine of the University of Peradeniya}

The undergraduate curriculum of the FMUP, where the reported studies were conducted, can be summarized as discipline (subject) based, and somewhat community oriented, where clinical and community health is learnt after the second year. Accordingly, basic sciences are taught in the initial phase followed by clinically oriented subjects. Students receive their clinical training at tertiary care hospitals and community health centres.

During the course of studies teaching and assessment is strictly discipline based. For example the structure and function of the heart and blood vessels is taught in year 1, anat- 
omy, physiology, biochemistry, and drugs in the management of heart disease in year 3 , while its disorders are dealt with during pathology in year 3 and 4 . The issue of diagnosis and management of heart disease is dealt with at different years from year 3 till year 5 . These departments are often not aware of each other's teaching programme leading to disorganised repetition of content. Sometimes individual teachers give mixed messages to students, thus students are left to make sense of this scattered information. The main method of teaching is the large group lecture. Teaching is often didactic. However, some departments have introduced innovative teaching and learning methods such as seminars, projects, assignments, etc. Teaching of behavioural sciences and communication skills are more or less limited to the psychiatry clerkship. So far, however, the non-lecture methods of teaching have not taken root in the faculty as a major form of instruction.

Teachers often express the idea that during secondary education the students have been trained by their teachers to learn basically through listening to lectures, taking down notes, which are often dictated to them and are later used to answer the questions. Thus, the conjecture that the students at entry are unable to learn on their own. Poor English language communication skills among a considerable number of students are thought to add on to this problem. Thus, the majority of the teachers argue that these Sri Lankan students, unlike their western counterparts, are relatively immature and inexperienced to learn on their own grasp fundamental concepts in medicine. Furthermore, in keeping with the Asian tradition of respecting one's teachers and elders, teachers want to have complete control over the students. This tradition is said to be common in Middle Eastern countries as well (Tekian, 1997). Thus, the curriculum is developed, executed and assessed at the pace decided by the teachers. Lack of coordination and integration seems at odds with the mission statement.

With respect to assessment, emphasis is on end of course, summative assessments. However, some departments conduct in-course assessments; often the contribution of incourse examination marks is $30 \%$ of the total. In addition each of the pre-clinical, paraclinical and clinical subjects are assessed by essay questions and viva voce examinations, whereas three of the departments have assigned a score for project work. Furthermore, most of the disciplines have incorporated objective assessment methods such as MCQs, objective structured practical examinations (OSPE) and objective structured clinical examinations (OSCE) to their schemes of assessment. Assessment in the final year includes a "long case", a case presentation by the student and followed by a discussion with the examiners lasting for about 45 minutes, and examination of a varying number of short cases depending on the speciality.

\section{The existing curriculum at Faculty of Medicine, University of Peradeniya} reviewed using the SPICES model

The SPICES model, described by Harden, Sowden, and Dunn (1984), is increasingly used by medical educationists to evaluate, review and compare the features of a medical school curriculum (Jayasinghe, 2002; Tekian, 1997). This model comprises six issues presented on a spectrum between two extremes of a continuum. At one end is the conventional curriculum and the other end an innovative one. The mnemonic SPICES is derived from the first letters of the innovative end as shown in Figure 2.1. Table 2.4 describes the educa-
tion strategies of the SPICES model. 
leacher centred Information gathering

Discipline based

Hospital based

Standard progranme

Apprenticeship/Opportunistic

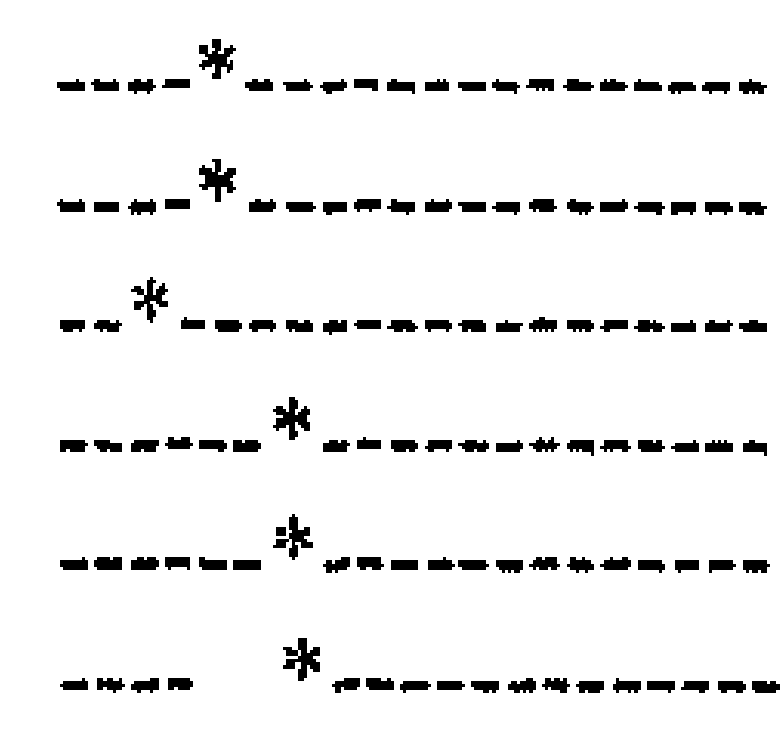

Student centred

Problem based

Integrated

Community based

Electives

Systematic

Figure 2.1. The curriculum of the Peradeniya Medical School on the SPICES spectrum (based on Harden. et al. 1984)

Table 2.4. Description of the SPICES model (derived from Harden et al., 1984)

\begin{tabular}{|c|c|}
\hline Innovative & Conventional \\
\hline $\begin{array}{l}\text { Student Centred: learner under the guidance of the } \\
\text { teacher takes more responsibility for his/her own } \\
\text { learning. Undertake to do assignments, self- } \\
\text { assessments. Selects the learning resources and de- } \\
\text { cide on the pace of learning. Learning tends to be } \\
\text { active. }\end{array}$ & $\begin{array}{l}\text { Teacher centred: Teacher is the directing agency. } \\
\text { Teachers decide on lecture topics, tutorial content, } \\
\text { organization and pace of learning. Teacher presents } \\
\text { subject matter in a logical order. Learning tends to be } \\
\text { more passive. }\end{array}$ \\
\hline $\begin{array}{l}\text { Problem based: Clinical problems are used as a } \\
\text { stimulus to learning. Basic concepts are learnt within } \\
\text { a given problem and learning is active as opposed to } \\
\text { passive gathering of in formation. }\end{array}$ & $\begin{array}{l}\text { Information gathering: The emphasis is on imparting } \\
\text { to students a large body of basic science and clinical } \\
\text { knowledge. Main objeclive is the acquisition of facts, } \\
\text { concepts and principles. A large number of lectures } \\
\text { and tutorials are conducted to transmit knowledge. }\end{array}$ \\
\hline $\begin{array}{l}\text { Integrated: Organize teaching learning in an } \\
\text { Interrelated manner to unify subjects frequently } \\
\text { taught in separate academic courses. }\end{array}$ & $\begin{array}{l}\text { Discipline based: Teaching learning on the basis of } \\
\text { subjects. Each discipline is taught at a particular phase } \\
\text { of the course allowing the student to synthesize and } \\
\text { apply to the clinical problems. }\end{array}$ \\
\hline $\begin{array}{l}\text { Community based education: providing training in a } \\
\text { community setting enabling students to experience } \\
\text { health problems in the context of the community. } \\
\text { Students work with health personnel to promote the } \\
\text { health of their patients. }\end{array}$ & $\begin{array}{l}\text { Hospital based: Students spend most of the time with } \\
\text { institutionalized patients experiencing a specialized } \\
\text { form of patient management. Hospital provides a } \\
\text { more concentrated form of experience of disease. }\end{array}$ \\
\hline $\begin{array}{l}\text { Electives: Learning opportunities to study a subject } \\
\text { in depth and gives flexibility to the learner and en- } \\
\text { courage involvement in research. }\end{array}$ & $\begin{array}{l}\text { Standard programme: All learners follow a standard } \\
\text { predetermined programme. }\end{array}$ \\
\hline $\begin{array}{l}\text { Systematic: teaching/learning sessions organized to } \\
\text { cover all the important topics, thus, students will } \\
\text { have equal opportunitics. }\end{array}$ & $\begin{array}{l}\text { Opportunistic or Apprenticeship based: } \\
\text { Depending on the availability of "good cases" in the } \\
\text { wards, those topics are covered. }\end{array}$ \\
\hline
\end{tabular}

Though the general trend in most places of the world is to re-structure the curriculum and move towards the right side of the spectrum, the curriculum of the FMUP appears to be less student-centred and integrated. It occupies a position midway between the left and the centre. Figure 2.1 depicts the existing positions of the Peradeniya Medical School within the SPICES spectrum, identified using a visual analogue scale.

In keeping with the current trend in medical education, it is desirable for the FMUP to revise its curriculum and move towards the right end of the SPICES model. It is also imperative to improve the teaching skills of the teachers in order to improve the quality of learning. In addition, the institution should introduce a quality assurance system for greater efficiency and productivity.

Although debate on need for and ways of curriculum reform has been intense at the FMUP, comparable major changes in the shape and substance of medical education have not occurred in actual practice. Most of the innovations in teaching and assessment have occurred within the context of the traditional curriculum. Hence some curriculum change 
has taken place over time at the FMUP though it has not been well coordinated and was not based on empirical evidence such as the performance of graduates or the changes in learning behaviours of undergraduates. The curriculum changes that have occurred over the years at FMUP amount to intra-departmental or if at all inter-departmental efforts. Structural changes proposed by faculty curriculum committee since 2003 are yet to be accepted by the staff members. Furthermore, the tradition of teachers' commitment to adhere to an acceptable "curriculum cycle" of quality assurance is also yet to be developed in most institutes.

Finally it is reasonable to state that the commitment towards conducting educational research in the context of Higher education in Sri Lanka is far from ideal (Lakshman, 1998). The practice of linking up educational policy decisions with research findings is yet to evolve in the country. Furthermore, no in-depth study of the learning process of undergraduate medical students in Sri Lanka has been reported so far. The situation with respect of educational research during the 1990s in the FMUP is no different to the national (Sri Lankan) scene. Unfortunately the learning process of medical students of the FMUP has hardly been the focus of research. "Has the existing learning environment promoted learning to learn skills and self-regulation of learning?" remains unanswered. At the faculty board meetings some academics express the opinion that a fair proportion of even the advanced medical students are unable to self-regulate the learning process. On the other hand some others discard those comments as anecdotes. The existing ground situation at FMUP is one of persistence of anecdotal evidence due to lack of empirical evidence. Undoubtedly the findings of study strategy research can be of immense help in the curriculum development process.

As described in Chapter 1, I have witnessed the intense debate on the need to reform the curriculum in order to facilitate problem solving, critical thinking and communication skills and encourage active learning among the graduates. However, in the face of dwindling financial and other resources leading to a reduced commitment of the staff, educational reforms were sidelined. Yet, at the very moment new pressure toward change is building up. Although the Ministry of Health employs all the medical graduates at present, from 2009 jobs are no longer guaranteed in the government sector for all, which also means that the country will have to prepare its graduates for the international market. Thus, the medical school teachers are faced with the challenge of reforming curricula and the institutional environments to produce graduates who are capable of self-regulating the learning process and pursuing professional development. In this background the present investigation was aimed at exploring different aspects of learning among medical undergraduates such as study habits, conceptions of learning, motivations, perceptions and their interrelationships. Therefore, the studies described in the empirical chapters are expected to provide scientifically valid data that could lead to a productive dialogue and informed decision-making with a view to improve the academic teaching and learning processes in the FMUP, Sri Lanka. 


\section{Student learning in higher education}

\section{Introduction}

\section{Learning as a complex phenomenon}

'Real learning' is an extremely complex phenomenon. Bandura's (1986) social cognitive theory emphasises the role of the personal, behavioural and environmental factors in learning. Learning is due to a number of interacting variables. Personality dimensions are an integral part of students' approaches to learning (Geisler-Brenstein, Schmeck, \& Hetherington, 1996). Bandura has provided the theoretical base for the development of a model of self-regulated learning in which personal, contextual and behavioural factors interact in such a way that students can exercise control over their learning and at the same time limits for self-direction are set. The notion that cognitive and personality functioning are integrated is not new. Curry's (1983) 'onion model' suggests that learning behaviour is fundamentally controlled by the central personality dimensions (inner layer of the onion) that are translated into information processing dimensions and then manifested through interaction with environmental factors (the outer layers of the onion). Hounsell (1997) argued "it is therefore misleading and unjust to attribute poor academic achievement to inherently 'weak' or 'unmotivated students' as interest, commitment and approach are products of the interaction between student and situation" (p.253).

Educational research has provided a great deal of evidence about factors associated with student learning. In the 1960's and early 1970's the focus of research in higher education was on student selection and prediction. It is also evident that a considerable number of studies on study strategies in higher education has focused on cognitive processing strategies and motivation (e.g. Biggs, 1987; Entwistle \& Ramsden, 1983; Schmeck, 1983). More recent research has examined the processes of learning, using the concepts of learning derived from qualitative studies. Brown (1987) has drawn attention to the importance of metacognition. The term metacognition refers to learners' views and beliefs about learning and to the active regulation of their learning processes (Flavell, 1987), thus emphasizing the active role of the learner. Newer inventories that were designed to assess the learning processes of students have made these dimensions explicit and emphasized their value in promoting reflection on study processes (Pintrich, 2004; Vermunt, 1998).

The development of expertise involves developing domain specific competence in addition to epistemological development and a shift towards more self-regulated and meaning directed learning. Recent research on medical education has shown that a flexibly organized and easily retrievable knowledge base is in fact a critical factor in medical diagnostic expertise (Lindblom-Ylänne, 1999). Thus, experts' diagnostic superiority was attributed to their knowledge organization, not to variables related to the problem solving process. Expertise is not seen as an end state but as something that needs to be developed continuously (Sternberg, 1998; Van der Vleuten, 1996).

As stated by Entwistle (1997), "educational research can be seen as careful, systematic attempts at achieving a better understanding of the educational process, with the aim of improving its effectiveness" (p. 3). This means our task is to describe more clearly how learning takes place in higher education, and to point out how teaching and assessment affect the quality of that learning.

With the explosive growth of the number of participants in higher education, in the recent past the attention for the quality of learning has grown as well. It is also believed 
that deep level transformation of knowledge is associated with qualitatively better learning (Lindblom-Ylänne, 1999). Therefore, the quality of learning has become a key issue for researchers (e.g. Wagner \& McCombs, 1995). A number of researchers have analyzed the qualitative changes in student learning within the context of the introduction of specific educational innovations (e.g. Nijhuis, Segers, \& Gijselaers, 2005; Vermetten, Lodewijks, \& Vermunt, 1999b).

\section{Conceptions of learning and knowledge}

In the view of most cognitive psychologists learning is a product of the interaction among what learners already know, the information they encounter and what they do as they learn. Therefore, learning can be thought of as created out of the learners' point of view, their knowledge and approaches to learning and the information they encounter. This is in line with Bednar, Cunningham, Duffy, and Perry's (1991) view that learning is not a passive, knowledge-consuming and externally directed process, but an active, constructive and self-directed process in which learners build up internal knowledge representations. It encompasses the idea that knowledge is constructed by the learner, learners consciously make decisions on what cognitive strategies to be employed and that learning involves qualitative restructuring and modification of schemata rather than just accumulation of new information in memory (Lonka, Joram \& Bryson, 1996). Learning thus becomes a matter of the individual constructing meaning and the learner is viewed as a very active participant of the learning process (Duffy \& Jonassen, 1992; Pintrich, 2004). As Schunk (1991) pointed out, students should be treated as active and creative agents in seeking and processing information rather than passive learners. A large body of research is in support of the notion of constructivism. At present it has become the dominant theory in education. According to constructivism, learners arrive at meaning by actively selecting and analyzing information to construct their own knowledge, through both individual and social activity (Biggs, 1996).

In addition, according to the student regulated learning model of Pintrich (2004), it is assumed that learners construct their own meanings, goals and strategies from the information available to them. Secondly, learners have the potential to monitor, control and regulate certain aspects of their cognitive activities, motivation, behaviour and some features of the learning environment. Thirdly, the learners set a goal or standard against which comparisons are made in order to assess progress of the learning process. Finally, it is also assumed that the self-regulatory activities play a mediator role between personal and contextual factors and actual achievement.

Teaching and learning have an interdependent relationship. Within the knowledge construction model, teaching is concerned with how to arrange the environment to enable learning to take place. Hence, Resnick (1987) indicated that the constructivist perspective is inconsistent with the traditional view of instruction as direct transfer of knowledge. Teaching is increasingly viewed as the facilitation of learning. In this context Shuell (1988) introduced the notion of 'teaching functions', which are psychological functions that have to be fulfilled for high quality learning to take place (Vermunt \& Verschaffel, 2000).

As noted by Perry (1970) and Lindblom-Ylänne (1999), college students' beliefs about knowledge change from that of a dualist (perceive knowledge as right or wrong) to a relativist (perceive knowledge as context dependent) perspective with academic progress. This was termed epistemological development among students and accounts for some of the differences in tackling academic tasks by novices and experts in a given field. 
Recent interest in process-oriented learning is based on the theoretical underpinnings of the notion of 'constructivism' and epistemological development.

Life long learning has become a necessity for every one in the present society. Therefore, in contemporary teaching greater emphasis is laid on active as opposed to passive forms of learning (Vermunt \& Verschaffel, 2000). One form of active learning is self- directed learning and this refers to the number and kinds of decisions that are taken by the learners' themselves or in co-operation with a teacher. Hence, in more active forms of learning, learners make their own time planning, they choose learning goals and activities on their own and they reflect on errors (Van Hout-Wolters, Simons, \& Volet, 2000). The second meaning of active learning is the amount of mental activity employed by the learner. Learners are supposed to be more motivated and interested when they can make decisions about their own learning and when their mental activity is challenged. It is assumed that an environment, which encourages active learning, also provides opportunities for students to develop their skills of learning.

\section{Conceptualising student learning}

Within the large body of research on 'student learning' there is much terminological and conceptual confusion. Different uses of the same concepts are common. Moreover, different terms with regard to learning are encountered in this thesis, thus, it seems appropriate to define and order them beforehand.

\section{Learning activities, approaches to learning, and learning strategies}

First, learning activities are those thinking activities that individuals employ to learn (Vermunt, 1996). Vermunt and Verloop (1999) further divide these learning activities into cognitive, regulative (metacognitive) and affective activities.

Cognitive processing activities These are the mental activities that learners engage in to process subject matter, which ultimately leads to learning outcomes. These can be internal activities (e.g. selecting main points; thinking of examples) or external activities (marking or underlining central concepts in their text books). Examples are: looking for connections between different parts of the subject matter; organizing knowledge into interconnected wholes; deriving the overall meaning of what has been studied; comparing information with personal experiences and connecting subjects with day to day phenomena; thinking of analogies; rehearsing subject matter; drawing up ones own conclusions based on facts and arguments; distinguishing between major and minor points; and reducing large amount of information by highlighting or making short notes (Vermunt \& Verloop, 1999).

Regulative activities These are the activities by which learners exert control over their learning processes. These are activities such as: planning a course of action to achieve a set goal; preparing a learning process by examining course characteristics as course content, assessment, etc.; monitoring whether the learning process proceeds in the right direction; adapting the study plan if necessary; testing oneself as to whether one understands or can apply the subject matter; and looking for gaps in knowledge, skills and attitudes by trying to answer questions or doing a practical. Activities such as evaluating and reflecting on how successful the plan was in terms of achieving learning outcomes by doing trial 
exams, thinking over the things that have happened in the learning process and thinking about learning, thinking about the learning activities that could be tried a next time, reflecting on the usefulness of cooperative studying etc., are also considered as regulative activities in any learning process (Vermunt \& Verloop, 1999).

Affective activities These are activities performed by learners to deal with the emotions that arise during learning. These activities create a mood that may have a positive, neutral or negative effect on the progress of learning processes. Examples are: motivating oneself; building up a willingness to learn; thinking of the negative consequences when one wants to quit learning; exerting effort; directing attention on task relevant work and coping with task irrelevant thoughts; attributing learning outcomes to stable versus unstable or controllable versus uncontrollable factors; deriving judgments about oneself; dealing with emotions such as fear, anger, stress, anxiety, and frustration; and building up self- confidence and commitment (Vermunt \& Verloop, 1999).

These learning activities constitute a main part of the learning process and learning processes can be understood as any series of mental actions directly responsible for learning outcomes (Vermetten, 1999). Learners spontaneously use specific patterns or combinations of learning activities to achieve their learning goals, and these combinations of learning activities are termed learning strategies (Vermetten, 1999; Vermunt \& Verloop, 1999).

Learning strategies These are defined as the specific combinations of learning activities that students employ to process subject matter to achieve their learning goals (Vermunt \& Verloop, 1999). In his empirical studies Vermunt (1998) found three cognitive processing strategies: (1) a deep processing strategy, combining learning activities as relating, structuring and critical processing; (2) a stepwise processing strategy, consisting of analyzing and memorizing learning activities; and (3) a concrete processing strategy, with concretizing and applying as dominant learning activities. With regard to regulating strategies, his findings indicated that the main distinction was based on internal versus external control. (1) The self-regulation strategy encompasses regulation activities initiated by the learner to regulate one's learning, e.g. orientation or planning of one's own learning process. (2) The external regulation strategy refers to relying on didactic measures like learning objectives, directions for studying, teacher dictated assignments, etc. (3) Lack of regulation is manifested when students notice they are unable to self-regulate their learning and also experience insufficient support of external control devices.

Entwistle (1997) and Marton and Säljö (1997) use the term approach to learning to describe the different ways of information processing adopted by students in reading an academic text, which they called deep and surface approach. The term approach encompasses the organizational aspect of learning described by Svensson (1997) and the intentional aspect of learning described by Marton and Säljö (1997). The individual student's approach to study at university has also been described in terms of surface and deep approaches (Marton \& Säljö, 1997). Research has revealed that it is the student's intention that promotes him of her to engage in a deep or surface approach. The third category, which is the "strategic approach", has been defined by Biggs (1993) and Entwistle (1997).

The defining features of an approach to learning are: the intentions of the learner (e.g. personal interest in understanding the underlying meaning or simply memorize to write at the exam); the type of learning activities one employs to comprehend subject 
matter; and the overall planning of the learning process. The two commonly reported approaches are: (a) deep approach- representing studying material for its own merit, typically out of personal interest, in search for basic meanings and structural interrelationships inherent in material and in interpreting material against personal knowledge structures and experience; and (b) surface approach - essentially studying to satisfy course demands and imposed assessment requirements, maintaining the components of studied material as discrete and unrelated both internally and personally and relying on verbatim memorization and reproduction. The third category, which is referred to as the strategic approach (Biggs, 1987), is an amalgam of the deep and surface approaches. Features of this approach are the intent to maximize performance and grades, allocating study time and effort in systematic and deliberate fashion and adopling deep and surface strategies according to what is judged optimal and efficient for attaining grades. Table 3.1 summarizes the defining features of the approaches mentioned above.

Table 3.1. Defïning features of each learning approach

\begin{tabular}{|c|c|c|c|}
\hline & Deep & Surface & Achicving \\
\hline Intention & $\begin{array}{l}\text { To understand ideas for your- } \\
\text { self }\end{array}$ & $\begin{array}{l}\text { To cope with course require- } \\
\text { ments }\end{array}$ & $\begin{array}{l}\text { To achieve highest possible } \\
\text { grades }\end{array}$ \\
\hline Process & Transforming & Reproducing & Organizing \\
\hline $\begin{array}{l}\text { Strategy } \\
\text { adopted }\end{array}$ & $\begin{array}{l}\text { Relating ideas to previous } \\
\text { knowledge and experience; } \\
\text { looking for patterns and un- } \\
\text { derlying principles; } \\
\text { checking evidence and relat- } \\
\text { ing it to conclusions; examin- } \\
\text { ing logic and argument cau- } \\
\text { tiously and critically; becom- } \\
\text { ing actively interested in the } \\
\text { course content }\end{array}$ & $\begin{array}{l}\text { Studying without reflecting on } \\
\text { either purpose or strategy; } \\
\text { treating the course as unrelated } \\
\text { bits of knowledge; memoriz- } \\
\text { ing facts and procedires rou- } \\
\text { tinely; finding difficulty in } \\
\text { making sense of new ideas } \\
\text { presented; feeling undue pres- } \\
\text { sure and worry about work }\end{array}$ & $\begin{array}{l}\text { Putting consistent effort } \\
\text { into studying; finding the } \\
\text { right conditions and mate- } \\
\text { rials for studying; manag- } \\
\text { ing time and eflort effec- } \\
\text { tively; being alert to as- } \\
\text { sessment requirements and } \\
\text { criteria; gearing work to } \\
\text { the perceived preferences } \\
\text { of lecturers }\end{array}$ \\
\hline
\end{tabular}

Source: Entwistle (1997, p. 19)

\section{Conceptions of learning and learning orientations}

Conceptions of learning are the knowledge; views, conceptions and beliefs people have about learning and thinking. Vermunt (1998) described "conceptions of learning" as consisting of students' beliefs about knowledge, thinking and learning, their conceptions of oneself as a learner, views on the task division between themselves, teachers, and fellow students in education, and conceptions of learning tasks and learning objectives. Learning conceptions form an important component of metacognition. He found that students' use of processing and regulation strategies was consistently associated with their learning conceptions and orientations. Learning conceptions and orientations together form the basis of engaging in a particular set of learning activities as well as analyzing one's own functioning as a learner.

Taylor, Morgan and Gibbs (1981), in defining the construct learning orientation, state that it encompasses all those attitudes and aims, which express the student's individual relationship with a course of study and the university. Based on interviews with Surrey University students, Taylor et al. (1981) identified a number of distinct orientations described by those students. These were: (1) an academic orientation, where intellectual curiosity is present. This orientation is further subdivided into extrinsic and intrinsic. An 
academic extrinsic orientation is where the objective is to obtain very high marks at exams and excel in studies, whereas students with an academic intrinsic orientation were studying at a higher level due to intellectual interest in the subject and were not so much syllabus bound. (2) A vocational orientation is where the primary motive is to undergo a good training to secure a job. (3) A personal orientation is where students have a high personal interest to advance themselves and their abilities. (4) A social orientation is where the objective of entering the university is to enjoy university life, to take part in many social activities so students would devote considerable amount of time for social activities. According to Beaty, Gibbs, and Morgan (1997), any particular student's orientation will be a mix of two or more of these orientation types. They state that an orientation does not assume any state or trait belonging to the student, but represents a quality of the relationship between the student and the course rather than a quality inherent in the student and so may change over time.

\section{Cognitive styles, learning styles and learning patterns}

The concept of 'style' is used in a variety of contexts such as sports, fashions, arts, media, and fairly widely in educational psychology. It is also pointed out that in these contexts the construct 'style' represents a distinct notion of coherent singularity. In most situations style is useful for identification. Similarly the term style has been used in educational contexts as a form of identity (Rayner \& Riding, 1997).

"Cognitive styles" are defined as a person's typical or habitual mode of problem solving, thinking, perceiving and remembering (Riding \& Cheema, 199l; Tenant, 1988). Cognitive styles represent ways of perceiving and not so much of learning. They are also considered as deep-seated aspects of personality and therefore as stable attributes. Since the 1970's popularity of this term showed a downward trend and was often replaced by the term "learning style". However, the two terms seem to differ in many ways. Cognitive styles are presented as bipolar constructs (e.g. 'verbalizer-visualizer', 'assimilator- accommodator') whereas learning styles usually contain several dimensions or elements each of which can characterize a student to a greater or lesser extent. Another difference is that cognitive styles refer to a theoretical academic concept, whereas learning styles are associated with a more practical educational application. In terms of stability cognitive styles are often seen as fairly fixed characteristics of an individual (Riding \& Cheema, 1991), while learning styles are not considered as unchangeable attributes but to express the effect of interaction between the learner and the learning content and context.

The literature extensively cites the work of Pask (1976) on learning styles and strategies. According to him, there are two typical ways of understanding: a comprehension learning style with a holist strategy (deductive reasoning, looking for comprehensive understanding and relations on a large scale) and an operational learning style with a serialist strategy (inductive reasoning, focussed thinking and step-by step processing from one idea to the next). Pask (1976) indicated that successful learners displayed a versatile learning style, the characteristic feature being the ability to employ comprehension and operation learning depending on the task at hand.

Among the several researches who have organized and ordered the different style constructs Curry (1983) did so by using the "onion" metaphor. According to him the inner layer of the onion represents the stable (learner) characteristics of the individual that do not interact with the environment directly. This represents the individual's cognitive personality style, a relatively permanent personality dimension as mentioned above. The middle layers of the onion accommodate the information processing style. Preferred 
learning strategies could be an example of this level. The outer layer of the onion represents the persons' instructional preference, which are the most modifiable constructs that interact directly with the environment. According to this model learning processes are basically controlled by the central personality dimensions, subsequently translated into information processing dimensions, and finally expressed through actual interaction with the environment.

In the literature the term learning style is mostly used in a narrow sense, for example in the sense of the learning activities students usually employ to learn (e.g. Moran, 1991). However, Vermunt (1996) used the concept of 'learning style' in a broader sense, and included students' learning conceptions and learning orientations in addition to processing and regulating activities. Entwistle and McCune (2004), trying to analyze the constructs discerned by factor analysis of different inventories, also stated that Vermunt (1998) used the concept learning style to describe different patterns of learning discerned by factor analysis. Thus the concept of 'learning style' is a coordinating concept, in which the interrelations among students' processing strategies, regulation strategies, conceptions of learning and learning orientations are united.

Recently, in an attempt to overcome the confusion caused by terminology, Vermunt and Vermetten (2004) suggested to use the term 'learning pattern' to describe this construct of 'learning style'. Moreover, Vermunt (1998) proposed a model in which students' conceptions of learning and learning orientations influence the way they process subject matter and regulate their learning processes. He therefore conceptualized conceptions of learning and learning orientations as more stable, individual variables, and learning strategies as more dynamic, changing aspects of learning. Finally, Vermunt (1996) states that learning style should not be conceived as an unchangeable personality attribute, but as the result of the temporal interplay between personal and contextual influences.

Research has repeatedly identified four such learning 'styles' or 'patterns' among students in a number of Dutch universities (Vermunt \& Vermetten, 2004). The first pattern is characterised by the use of relating and structuring, and critical processing strategies, self-regulation of learning processes, construction of knowledge as the conception of learning, and personal interest as learning orientation. This pattern was interpreted as meaning directed learning. The second, reproduction-directed learning pattern is typified by the use of memorising and rehearsing, and analyzing processing strategies, external regulation of learning processes, intake of knowledge as the conception of learning, and a certificate and self-test directed learning orientation. The third pattern was interpreted as an undirected learning pattern and is characterised by lack of regulation, an ambivalent learning orientation and co-operation and stimulating education as conceptions of learning. The fourth learning pattern is typified by the use of concrete processing strategies, use of knowledge as conception of learning and a vocational learning orientation and was called application directed learning.

\section{Research approaches to the study of student learning}

\section{A shift in research paradigm}

As cited by Entwistle (1997), Parlett and Hamilton (1972) criticized educational research for following a hypothetico-deductive method. They contrasted the traditional research paradigms with the procedures used by social anthropologists, who observe and question people in different cultures in a sincere attempt to understand their customs and beliefs. 
As opposed to the traditional approach, which explains student behaviour from the outside as a detached objective observer, the alternative approach seeks an empathetic understanding of what is involved in student learning derived from students' descriptions of what learning means to them and how they set about doing things. The alternative paradigm uses approaches to research rooted in phenomenology to realize its goals. Thus it involves both a shift in methodology and of perspective. As a result, the dominant research paradigm in higher education at present involves looking at the learning situation through the eyes of the learner, thereby providing insights to the teacher firmly rooted in the real-life situations in tertiary education. This approach represents the situation more fairly as an interaction between the characteristics of the student and the experiences provided by the institution. Thus, it presents a description of student learning from an unusual perspective - that of the student - and yet leads to important implications for teaching as well as for studying (Entwistle, 1997).

Recently, Richardson (2004) pointed out that since the current university has changed in many ways over the past decades and the students are also more heterogeneous in social, cultural and ethnic terms, the demand for qualitative studies has increased. In summary, the dominant research paradigm in higher education at present involves looking at the learning situation through the eyes of the learner by using interviews; open written questionnaires, observations and qualitative, interpretative analyses. This paradigm involves the European phenomenographic approach (e.g. Entwistle, 1997; Marton \& Säljö, 1976; Marton, Watkins, \& Tang, 1997; Vermunt, 1996), as well as American research on students' intellectual development (Perry, 1970; Schommer, 1990, 1998).

\section{Phenomenography}

Phenomenography is a research methodology used to identify the qualitative different ways in which people experience, conceptualize, perceive and understand phenomena in the world around them (Marton, 1981, 1986). In this method extensive interviews or written open questions are being used to obtain information on main areas of interest. As the first step a list of issues to cover the main areas of interest is prepared by the researcher in advance. Although guided by this predetermined framework, the interview is allowed to develop as a natural conversation and discussion. Sometimes the interviewer is seen to actively explore the experiences of the interviewee by probing deeply into how each subject perceives the phenomena of interest, in order to obtain a fuller description of what has happened. The interviewer has to play a neutral role and refrain from presenting opinions or ideas but at the same time conduct an interactive discussion. Then, with the permission of the interviewee, the interviews are tape-recorded and tapes transcribed in full. Analysis involves repeated reading of both the overall transcript and the comparable transcripts of all the students looking for consistencies and differences, which are later analyzed under various themes. The objective of this method is to frame and describe these qualitative differences in conceptual categories (Säljö, 1988) as well as to search for relan tionships both within and between these conceptions (Marton \& Säljö, 1997).

\section{Study strategies inventories}

An inventory is a questionnaire with statements that produce scores on a series of scales. Students rate the extent to which their learning activities, conceptions or orientations match a number of statements. It is common practice in educational research to study learning processes by administering self-report questionnaires or inventories. These questionnaires are increasingly used to obtain information about the strong and weak sides of 
students' learning patterns. At university level it is important to gather diagnostic information about the learning strategies and skills of learners as a group. This could then lead to decisions with regard to the aspects to be trained separately and/or to be emphasised during regular lessons (Van Hout-Wolters, 2000). At present, many such standardized inventories are available in English, for example, the Inventory of Learning Processes (ILP, Schmeck, Ribich, \& Ramanaiah, 1977), the Approaches to Studying Inventory (ASI; Entwistle \& Ramsdon, 1983), the Learning and Study Strategies Inventory (LASSI; Weinstein, Palmer, \& Schulter, 1987), the Motivated Strategies for Learning Questionnaire (MSLQ; Pintrich, Smith, Garcia, \& McKeachie, 1993), the Study Process Questionnaire (SPQ; Biggs, 1987), the Revised Approaches to Studying Inventory (RASI; Entwistle \& Tait, 1994), and the Inventory of Learning Styles (ILS; Vermunt, 1998). These questionnaires seem to differ in goal, subject matter, target groups, type of questions/statements, number of scales and items, fill-in time, reliability and validity. However, all of them try to gain insight into learners' cognitive and metacognitive learning activities by the leamers written retrospection (Van Hout-Wolters, 2000). There is a trend of construction of new questionnaires on the basis of items of different existing standardised questionnaires as well. In addition, some of the inventories have been translated into other languages and elaborated to be used in foreign countries (Ajisuksmo, 1996; Albaili, 1997). Ajisuksmo (1996) composed an Indonesian version of the $I L S$, called "Inventarisasi Cara Beljar" $(I C B)$ to measure learning patterns among Indonesian higher education students.

The $A S I$ contains scales on deep approach, surface approach, and achievement motivation. The $A S I$ has been used in a number of different cultural contexts. It has also being used widely to explore the learning approaches of medical students (Coles, 1985; Emilia \& Mulholland, 1991; Jayawickramarajah, 1995; Martenson, 1986; Stiernborg \& Bandaranayake, 1996). The RASI contains scales in the domain of cognitive processing (e.g. deep approach, surface approach, strategic approach and apathetic approach) and study motivation and affection (e.g. active interest fear of failure, intention to excel and lack of direction). The $L A S S I$ contains scales not only in the domains of cognitive processing and motivation but also on metacognitive regulation (e.g. 'self testing').

Among the widely used instruments to investigate university students' approaches to study, Biggs's (1991) SPQ also needs special mention. The $S P Q$ has been used in a number of western studies and in a few studies conducted in south East Asia (Biggs, 1991, 1992, 1993; Hattie \& Watkins, 1981; Hiliard, 1995; Kember \& Gow, 1990; Stokes, Balla, \& Stafford, 1989). Biggs makes a distinction between three learning strategies deep, surface and strategic, each corresponding with a particular study motive: intrinsic motivation, instrumental motivation, and achieving motivation, respectively. Biggs (1978) states that the $S P Q$ is based on motive-strategy congruence theory with three motive-strategy "packages".

Lonka and Lindblom-Ylänne (1996) combined different parts of a number of existing inventories to incorporate cognitive, regulative, metacognitive and motivational components of student learning in one study. However, there appears a scarcity of research investigating the relationships between cognitive and regulative learning strategies, conceptions of learning and learning orientations. Against this background, Vermunt (1996) aimed at increasing the integration of existing conceptualizations of student learning and at linking metacognitive aspects of student learning to students' cognitive processing strategies and study motivation. According to the model of regulation of constructive processes proposed by Vermunt (1998), the way in which students process subject matter 
is most directly influenced by the regulation strategies they employ. Conceptions of learning and learning orientations also influence the processing strategies that students use, but their influence is supposed to be mostly indirect, via regulation strategies. That is to say that the way in which students regulate their learning process is to a great extent determined by their conceptions of learning and learning orientations.

Although this kind of measurement is common practice in educational research, it is important to acknowledge its limitations. A limitation of the method is that it is based on what students recall they do when studying rather than on what they actually do. Another limitation is the possibility of providing socially desirable responses (Stiernborg \& Bandaranayake, 1996). In the past decades both the student populations and university learning environments have changed considerably, thus Richardson (2004) expressed doubt on the content validity of some of the questionnaires for current student populations studying in innovative learning contexts.

Christensen, Massey, and Isaacs (1991) described inventories of learning/study processes as arising from three kinds of research base namely;

1. A-theoretical quantitative: which is a traditional quantitative approach using psychometric techniques such as factor analysis to develop inventories of learning and study approaches (Biggs, 1987; Christensen et al. 1991; and Entwistle \& Ramsden, 1983 are cited as examples of this tradition).

2. Theoretical qualitative: that have been derived from a theoretical base, using recent work in cognitive psychology as a theoretical underpinning that is exemplified in the work of Schmeck et al. (1977) and Weinstein et al. (1987).

3. A-theoretical qualitative, which relies on the analysis of students' approaches as they undertake particular tasks (Marton \& Säljö, 1997; Svensson, 1977).

Biggs (1993), in discussing the role of theory in the development of learning process inventories, was of the view that the above categories are arguable. He claimed that the inventories developed by Biggs and Entwistle cannot reasonably be described as 'atheoretical', because each in fact has a substantial theoretical foundation. The $M S L Q$ was also based on a theoretical model, which incorporated an information processing and a social-cognitive perspective on motivation. Similarly, the $I L S$ has a rich theoretical base, a psychometrical validation and items derived from qualitative studies, and hence it is an example of qualitative quantitative mix.

Therefore, rather than contrast 'quantitative' and 'qualitative' theories of student learning research, Biggs (1993) argued for consideration of the Entwistle and Waterson's (1988) distinction between two main theoretical positions as the source of current learning /study process inventories. That is to go by the information processing (IP) position deriving from cognitive psychology, as exemplified by Schmeck et al. (1977) and Weinstein et al. (1987), and the student approaches to learning (SAL) position derived from qualitative research on student learning, underlying the $A S I$ and the $S P Q$. The model proposed by Curry (1983) can also be used to differentiate learning style inventories by the extents to which they reflect personality characteristics, information processing characteristics, or preferences for different types of instruction.

Recently, Entwistle and McCune (2004) have analyzed the conceptual bases of six of the most well documented inventories and stated that over the years succeeding generations of inventories have built on earlier ones. Several well-documented inventories are available for researchers now, to obtain data on study strategies and associated constructs of university students. It is apparent that the newer inventories were built on the 
earlier ones and there is considerable overlap among them. Several of these inventories have been translated to other languages and validated in different cultures (Boyle, Duffy, \& Dunleavy, 2003)

The $I L S$, originally developed in Dutch, is available in English. The Indonesian version of the $I L S$, the $I C B$, has been administered to university students from a number of disciplines in Indonesia (Ajisuksmo, 1996; Ajisuksmo \& Vermunt, 1999). Moreover, the $I L S$ has been administered in different cultures and the interrelationships between the four main aspects of learning namely, the cognitive processing activities, regulation activities, conceptions of learning and learning orientations have been documented (Vermunt \& Vermetten, 2004). The literature further reveals that the $I L S$ has been administered to undergraduate students (including medical students) in a number of European countries in order to explore various aspects of learning (Strømsø, Grottum, \& Lycke, 2004).

The characteristic feature of the newer inventories such as $I L S$ and the $M S L Q$ is that these have incorporated metacognitive aspects of learning and thus explicitly emphasize self-conscious reflection on studying (Entwistle \& McCune, 2004). It was seen that some items of these inventories explicitly assessed the beliefs and knowledge about learning as well as the ways of monitoring, regulating and reflecting on their learning. Entwistle and McCune also highlighted the fact that, overall, the $I L S$ has a strong focus on students' regulation of their learning, whether it is self-regulated, externally regulated or lacking regulation altogether. In addition, an advantage of the $I L S$ is that it could be used partly as a research tool and partly to allow students to reflect on and develop their ways of learning (Ajisuksmo, 1996; Entwistle \& McCune, 2004). The ILS, in particular, has recognized the value of collaborative learning where as most of the earlier inventories describe studying as a solitary activity (Entwistle \& McCune, 2004). Since the societal demand on students to be able to regulate their own learning is becoming more and more prominent, it was important to select an inventory that includes the regulatory aspects of learning. The $I L S$ was thought to be an appropriate research tool for the proposed Sri Lankan study and was selected for the purpose of developing a culturally acceptable test instrument. In Chapter 6 the $I L S$ will be described in more detail.

Different foci can be distinguished in research regarding the measurement of learning processes (see Biggs, 1993). The perspective that is taken when assessing learning processes varies from very specific contexts (such as reading a text or problem solving) to very general contexts (such as students' usual way of studying). If a specific context is considered, the assessed construct presumably represents the learning processes actually undertaken. Examples of such studies are Marton and Säljö (1997), Svensson (1977), Eley (1992), and Beishuizen, Stoutjesdijk, and Van Putten (1994). If a more general context is considered, the construct assessed is more likely to represent the learner's predisposition to learn in a certain way, which is not linked to a specific situation. Most learning inventories exemplify this latter meaning, as they ask students how they usually go about learning (e.g. Biggs, 1987; Entwistle and Ramsden, 1983; Schmeck, 1983; Vermunt, 1998; Weinstein et al. 1987). Nevertheless, with the right instructions to the respondents, these questionnaires can also be used to measure learning processes at a more specific level of context, aiming at learning processes actually undertaken. 


\section{Research findings on student learning}

Consistency and variability in students' learning strategy use

A number of researchers have argued that learning strategies and approaches are context specific and are brought into action in accordance with the specific circumstances of the teaching learning context (Entwistle \& Ramsden, 1983; Trigwell, Prosser, \& Waterhouse, 1999; Vanderstoep, Pintrich, \& Fagerlin, 1996). Others argue that learning strategies are part of a person's predisposition to learn in a consistent way like a personal style (Schmeck, 1983). However, Vermunt and Vermetten (2004), in addressing the issue whether the learning strategies are more like a consistent individual learning style or whether they are flexible and adaptive towards a specific situation, state that there is a person bound component and a context bound component in students' use of learning strategies. Empirical evidence suggests that the issue of consistency versus variability of learning strategies does not yield an "or-or answer but an and-and answer" and that learning strategies differ from each other in their degree of variability (Vermetten et al. 1999b). These authors found that the use of a memorizing processing strategy was relatively stable while concrete processing and lack of regulation showed a relatively high sensitivity for the course context. Vermetten, Lodewijks, and Vermunt (2002), in another study, provided evidence that educational reforms in a Dutch university aimed at providing a student oriented learning environment failed to influence the reported learning strategies in the direction of more deep and self-regulated learning during the first two years of undergraduate education. Explaining the results they stated that the instructional measures might not have been powerful enough to create more deep level learning strategies. In the same study they also found that the student groups with different learner characteristics used instructional measures in different ways to suit their own habits, ideas and preferences of learning. These results indicate that instruction has an indirect effect on reported learning strategies instead of a direct influence.

\section{Dissonance in student learning patterns}

In most of the studies on student learning, the learning patterns found among students comprise learning activities in line with their views on learning and their learning motives and goals. They indicate normal patterns, interpretable from a theoretical point of view. However, learning patterns identified in some other studies with the $I L S$ have shown the emergence of deviant learning patterns (Vermunt \& Verloop, 2000; Vermunt \& Minnaert 2003). Empirical studies have also reported that for some groups of students the typical, 'normal' patterns of interrelationships between learning components (processing strategies, regulation strategies, conceptions of learning and learning orientations), do not show up (Meyer, 1991; Vermunt \& Vermetten, 2004). This refers to the phenomenon of dissonance. The 'normal' pattern of factor loadings as described by Vermunt and Verloop (2000), has scales within a component mostly having loadings on different factors. For example, within learning conceptions, construction of knowledge has its highest loading on the meaning directed learning factor, while intake of knowledge loads highest on the reproduction directed factor. Stimulating education and cooperative learning load highest on the undirected factor and use of knowledge on the application directed factor. Another feature typical of the 'normal' pattern of factor loadings is that each factor is composed of elements of the four different learning components (Vermunt, 1998). In this respect, Vermunt and Verloop (2000) reviewing the evidence for dissonance in studies that used 
the ILS or one of its versions as one of the instruments identified five different patterns of dissonance, which do not conform to what is expected theoretically. These are: (1) lack of differentiation within learning strategies, conceptions and orientations; (2) lack of integration between learning strategies, conceptions and orientations; (3) lack of the application directed learning pattern; (4) incompatibility of the learning strategies, conceptions and orientations; and (5) missing elements from learning pattems.

\section{Developments in student learning patterns over years of schooling}

A development theory could be derived based on the results of studies with the ILS in several educational contexts $i . e$. junior secondary education, senior secondary education and different stages of university education. Vermunt and Vermetten (2004) reported that the $I L S$ was administered to first, second and third years of secondary education and those in the upper phase of secondary education in the Netherlands by a number of researchers. They refer to a study in which the $I L S$ was administered to 984 students in the first year of secondary education (age about 12 years). Results of factor analyses on these data have yielded four factors. A characteristic feature of these factors, as opposed to those described by Vermunt (1998) with the first year university students, was that the factors demonstrated high loadings on a number of scales within one component of learning (processing strategies, regulation strategies, learning conceptions and learning orientations). For example, the first factor had high loadings of four of the five processing strategies, factor 2 had high loadings of three of the five regulation strategies and factor 3 had high loadings of four of the five conceptions of learning and learning orientations. Thus, there was no clear differentiation within learning components.

Vermunt and Vermetten (2004) review other studies that found very comparable results with large groups students of first, second and third year of secondary education (age 12-14). When the ILS was applied to students in the upper phase of secondary education, a factor structure resembling the structure found in tertiary education could be observed. They concluded that interrelations between ILS domains were stronger than among first year secondary education students, but not as strong as in tertiary education. In addition, Vermetten et al. (1999a) found, in their study with first and second year university students that the factor structure became stronger, more coherent among the different learning aspects in the advanced students. Vermunt and Vermetten (2004) observed that the factor structures discerned by studies with junior secondary students failed to reveal more focused and stronger relations between learning components when compared with those of senior secondary students and first year undergraduate students. On the other hand, Severiens (1997) has put forward an explanation opposing the former development hypothesis, which is called a context hypothesis. Her research with the ILS in adult secondary education showed a different learning pattern and she brought up the learning context as an explanatory factor. The observation that the factor structure of the upper phase of secondary education appears to fall back into pieces during the first year in the university contradicts the developmental hypothesis. Therefore, finally Vermunt and Vermetten (2004) conclude "that the development hypothesis holds true for students progressing within one type of education, while contextual hypothesis explains the disintegration of factor pattern when there is a change in the context such as a change from higher secondary to first year in the university". Therefore, diffuse factor pattern could herald a period of a new type of education.

According to them diffuse factor patterns could point towards an interesting developmental phenomenon and they postulate that ones' development as a learner proceeds 
along this line of differentiation within learning components. Another developmental line concerns increasing associations among the learning strategies students use and their learning orientations and conceptions of learning.

Another developmental model, which predicts various stages in academic learning, was described by Alexander, Jetton and Kulikowich (1995). This was called the Model of Domain learning (MDL). According to this model, three stages of development in learning in any field of study are the acclimation, or naïve learning to a stage of competence and then to a stage of proficiency or expertise; however, relatively few students achieve the stage of expertise. This model hypothesizes that at the beginning of learning in a new domain, students show low deep-seated individual interest, and have fragmented and incohesive domain knowledge. Therefore, they rely extensively on their strategy use to compensate for gaps in domain knowledge. As the students become more and more competent it is expected that the interest as well as the organized nature of domain knowledge will grow and reinforce one another. Alexander, Murphy and Woods, (1997) examined the validity of the model in a study on changes in learning during one academic course among premedical students and noticed that students' domain knowledge as well as their interest increased. They also observed a qualitative shift in strategy use with the increase in domain knowledge. Therefore, the MDL is considered as a multidimensional model in the sense that the changes are not only predicted within each variable but also with respect to their interrelatedness. This MDL model acknowledges a continuous interplay of cognitive and non-cognitive (affective) factors in the development of expertise and states that as a result of more coherent knowledge and greater motivation, a qualitative improvement in strategy use can be expected.

\section{Personal influences on student learning}

The two domains related to the adoption of certain learning strategies are the contexual domain and the personological domain (Vermetten, Vermunt, \& Lodewijks, 1999a). Ramsden's (1988) model of learning in context and the tetrahedral framework for learning described by Brown, Bransford, Ferrara, and Campione (1983) have demonstrated the involvement of the learner and the context in the adoption of learning approaches and strategies. The personological domain consists of the cognitive, affective and metacognitive or regulative aspects of learning as well as variables such as age, gender, personality traits, and academic locus of control as a means to explain individual differences in learning among students (Ajisuksmo, 1996; Busato, Prins, Elshout, \& Hamaker (1998); Drew \& Watkins, 1998; Severiens \& Ten Dam, 1997; Stiernborg \& Badaranayake, 1996).

With respect to conceptions of learning, Schommer (1998) reported that age affects individuals' beliefs about knowledge in unique ways. Richardson (2004) reported that older people obtain lower scores than younger people on measures of surface approach. Very recently, Vermunt (2005) stated that older students show more characteristics of meaning directed learning and they are less certificate oriented in their learning orientation. Advancement in age is invariably associated with a larger amount of life experiences which learners bring with them to the learning situation and with changes in learning motivation and learning ability, finally resulting in greater inclination towards meaning oriented learning.

Associations between students' gender and learning strategies have been studied by several researchers. Stiernborg and Bandaranayake (1996) reported that two subscales underlying meaning orientation showed significantly higher scores for males among medical students in a Australian university. Severiens and Ten Dam (1997) studied the 
relation between learning styles and gender and reported that men on average scored higher than women on undirected learning, while the reverse was true for reproduction directed learning. Recently, Mattick, Dennis, and Bligh (2004) studied the relations of medical students approaches to learning with personal characteristics such as age and gender, and reported that although age had no relationship with the reported approach, male students showed a positive association with surface strategies. Vermunt (2005) showed that female students attach greater value to cooperative learning than male counterparts, although he has not found consistent relationships between learning patterns and students' gender.

It is also possible that students' attempt at entry (whether the student secured admission to medicine in the first, second or third Advanced level examination attempt) has a bearing on the learning pattern. Senanayake and Weerasinghe (1996) analysed the selection examination scores and students' performance in the second MBBS examination, of a batch of medical students at the university of Peradeniya. They reported though the selection examination score had no correlation with their performance in the medical faculty, the attempt at entry had a bearing on his/her academic performance.

The time spent on studying or the independent study time was another variable that has been studied. In previous studies, Parer and Benson (1989) as well as Svenson (1977) have not found a significant relationship between time spent on studying and an approach to learning. However, Kember, Jameison, Pomfret, and Wong (1995) found that more hours of study time combined with surface approaches to learning, are an inefficient way of learning resulting in poor grades among a group of undergraduate engineering students in Hong Kong.

Academic locus of control may be another influencing variable. Since internal locus of control is a powerful motivating factor, a positive correlation with meaning directed learning elements is anticipated. Drew and Watkins (1998) found a negative association between internal locus of control and a surface approach among a group of Chinese university students.

\section{Relations between student learning and academic performance}

Learning is seen as an active process in which the learner synthesises, modifies and utilises the conceptions in a subject domain to interpret situations and act in them. It is assumed that the quality of the learning activities that the students employ, determines to a large extent the quality of the learning results they achieve. The use of learning activities makes the individual's knowledge base or repertory of skills and attitudes change (e.g. Trigwell \& Prosser, 1991; Vanderstoep, Pintrich, \& Fagerlin, 1996).

Contrary to the former view, traditional tests are often used to measure learning outcomes in students (Vermunt, 2005). The relation between learning activities and domain specific learning outcomes has been the focus of many studies. However, one must bear in mind that the exam achievements reflect a small portion of the learning results and that it is not always a true representation of the thinking activities undertaken by the learner. Sometimes student scores well with memorising alone depending on the way learning outcomes are measured. A matter of concern has been to what extent the exams in the undergraduate courses capitalise on the various learning activities that students employ.

Academic learning outcomes have been the focus of extensive research over the years and a number of factors and variables have been found to influence the achievement outcomes. Some researchers have examined the associations between different inventory scale scores and the examination performance of students (Arnold \& Feighny, 1995; Lei- 
den, Crosby, \& Folmer, 1990; Lindblom-Ylänne \& Lonka, 1999; Stiernborg \& Bandaranayake, 1996). The results of these studies have been generally consistent, although the correlation coefficients were small. According to the literature, meaning orientation and its subscales are generally positively related to academic performance, while the reproducing orientation is mostly negatively associated with academic performance. Busato et al. (1998) computed the correlation between $I L S$ factor scores (learning style scores) and study success in terms of number of study points for Psychology students and reported that undirected learning was consistently negatively related to study success. Meaning directed learning showed a positive association with performance while the reproduction orientation and application directed learning pattern showed no relation. Boyle et al. (2003) reported the same findings with the $I L S$ in a British higher education setting. Meyer (2000) observed that 'dissonant' learning patterns (learning patterns defined by incoherent learning strategies, conceptions, and orientations) were associated with low exam performance. Recently, Vermunt (2005) has analysed the associations between learning patterns derived from the $I L S$ and academic performance of undergraduates from seven academic disciplines (psychology, law, arts, sociology, economy, econometry and management information science) and shown that most elements of meaning directed learning were positively related to academic performance. He also reported that the pattern of relations between mean examination score and elements of undirected learning remained consistently negative.

\section{The context of student learning and approaches to teaching}

\section{The context of learning and its influence on student learning}

The learning context consists of the learning tasks, the teaching, the assessment, the course organization and the wider educational context. At present, there is a substantial body of evidence on the effect of teaching methods, learning tasks, assessment demands and workload on student approaches to learning. Christopoulas, Rohwer, and Thomas (1987), Gow and Kember (1990) and Ramsden and Entwistle (1981) suggested that the learning approaches students adopt are to some extent shaped by the teaching, assessment, and course organization, which also supports the context specificity of strategy use. Recent literature reveals a strong association between teaching characteristics and the ways in which students study (Biggs, 1999; Trigwell \& Prosser, 2004). Hence, the academic departments do have a responsibility for the efficiency of learning achieved by their students.

The use of reproductive approaches to learning by students has been demonstrated to correlate with factors such as: high workloads (Dahlgren, 1997; Gow \& Kember, 1990); surface level assessment demands (Entwistle \& Ramsden, 1983; Thomas \& Bain, 1984); low levels of intrinsic interest in the course (Fransson, 1977); and lack of freedom in the learning environment (Boekaerts, 1997; Ramsden \& Entwistle, 1981). Godfrey (1995) pointed out that even in innovative and forward looking medical schools across the world, much of the good intention is neutralised by the powerful steering effect of a traditional assessment system. Fransson (1977) concluded on the basis of his study on students' text processing, that in order to promote a deep approach, one should foster the students' own interest and at the same time eliminate the factors that lead to a surface approach, namely irrelevance, threat and anxiety. Eley (1992) found variability in learning strategies employed in two different courses conducted concurrently. Students also re- 
ported on the perceived learning environment. A link between frequent use of surface strategies and high performance demands on students at examinations was shown.

Trigwell and Prosser (2004) pointed out that students' perceptions of the learning environment is a factor which influence the use of study strategies and thereby the quality of learning outcomes. In the past, several researchers have utilised questionnaires as a means to measure students' perception of various aspects of the learning context (Eley, 1992; Trigwell \& Prosser, 1991). Examples are the Course Perceptions Questionnaire (CPQ; Entwistle \& Ramsden, 1983), the Course Experience Questionnaire (CEQ; Ramsden, 1998), and the Inventory of Perceived instructional activities (IPIA; Vermetten et al. 1999a). The course experience questionnaire, developed by Ramsden (1998) in Australia was based upon the $C P Q$ developed by Entwistle and Ramsden (1983) in Britain. The $C E Q$ consist of 25 items and was designed to provide an indicator of student perceptions of their learning environment or instructional- learning activities that have been in existence over their whole academic program. For each item the respondent has to indicate the degree of agreement or disagreement on a five point likert scale. According to Ramsden (1998), factor analyses on previous survey data had repeatedly yielded five dimensions: good teaching, clear goals and standards, appropriate assessment, appropriate workload, and generic skills (see Chapter 8 for a more elaborate description of this instrument).

Several levels of context detail have been studied. Ramsden and Entwistle (1981) studied the differences between academic departments in students' approaches to studying. Groups of students in different departments completed questionnaires, which measured their study strategies and also reported on their perceptions of the teaching context. The differences between academic departments were reported. Links between study approaches and perceptions could also be confirmed. Meaning orientation was associated with perceptions of 'good teaching' and 'freedom in learning'.

Lonka and Lindblom-Ylänne (1996) have studied the disciplinary differences between learning patterns of medicine and psychology students. The former group showed more externally regulated and reproduction directed learning. Similarly, Ajisuksmo (1996) studied the learning strategies of university students from disciplines such as business administration, law, engineering, and found that scores on some strategy scales were related to the field of study. A more general level of context detail is the type of curriculum that is adopted by a medical school. Researchers have compared the changes in learning aspects within a traditional curriculum and a Problem based curriculum (Coles, 1990; Martenson, 1986).

Several researches have investigated the changes in learning strategies at intermediate level of context detail such as the introduction of innovative programs, by administering inventories (Dolmans \& Wolfhagen, 2004; Nijhuis et al., 2005;Vermunt \& Minnaert, 2003). Ajisuksmo (1996) has studied the effects of a metacognitive training program on university students learning strategies. Vermetten et al. (1999a) studied the learning strategies reported by first year law students for each of the four different courses taken in the first year using the $I L S$. In order to find out the differences in the learning contexts teachers had been interviewed about instructional methods and assessment strategies in each course while study material was inspected. They found that the same group of students showed variability in the strategy use depending on the demands of the learning context of the different courses of law. Vermetten et al. (2002) have measured the effects of educational reforms, carried out under a student oriented education project at the Til- 
burg University on student learning strategies over a period of one semester at a general semester level and course specific level.

Implications of these research findings are modification of courses, conceptual development in teachers as well as organizational restructuring, having a direct effect on developing learning to learn skills among students. Hounsell (1997) cited the work of Eisenberg (1986) in the faculty of Medicine, University of Melbourne, where an orchestrated set of interventions in teaching and assessment in Anatomy led to a qualitatively better or more desirable learning outcome. Similarly, most of the educational benefits of problem-based learning are attributed to the changes in the approach to teaching and learning, which seems to be its cardinal feature.

An important contextual variable is the students' educational experiences and the nurturing environment. Learners bring certain attitudes and habits, which they have developed over the years of schooling and at home to the university. Some hold the view that certain learning strategies are crystalised by the time they enter university education. In Asian cultures, parents influence the learning behaviour of students by either encouraging or discouraging constructive learning through their actions, which are ultimately based on parents' perceptions of learning. Often parents do not encourage discovery learning. It is also speculated that the teacher parents (particularly if mother is a teacher) exert a high degree of control over their children's learning.

Another possible contextual variable would be the level of education of parents. As stated in the current chapter one's level of education has an impact on epistemological development. Accordingly, parents who have undergone higher education are more likely to consider learning as a constructive process and direct their children to use more constructive processing and regulation strategies. However, Ajisuksmo (1996) found very little evidence in support of the influence of parental education on the changes in learning strategies.

As reported in chapter 2, schools in some districts of Sri Lanka are poorly equipped in terms of human and material resources. Thus, it is possible that students who come from such schools have limited learning opportunities although it should not be the case. Such a situation may lead to the expression of different patterns of learning by those from privileged and underprivileged districts.

\section{Student learning in medical education}

The educational (approaches to teaching and assessment methods) as well as organizational environment of the medical school (which is influenced by administrative and financial regulations of the institution) seems to influence student learning. It is said that students' perceptions of teaching, assessment and course content, as well as the natural settings of academic departments, influence student behaviour. Hence the learning environment, which is directly influenced by the overall curriculum (teacher centred or student centred), teaching skills and perceptions of teachers, is an important determinant of student learning behaviour. Addressing the issue, as to how the educational and organizational environment of educational institutes (e.g. medical school) influence medical students' and teachers' behaviour, Genn (2001) indicates that the perceptions of the environment, designated as 'climate', are related to behaviour. Therefore, in bringing about a change in a medical school, one must pay adequate attention to the significance of environmental aspects. Examples of organisational changes fostering active learning are: providing facilities for students to work at their own pace such as improving access to learning resources off faculty hours, investing on infrastructure development i.e. good library 
facilities, introducing skills laboratories, making the administration more learner and staff friendly and investing on staff development for better implementation of proposed innovations. Since the learning environment has been viewed as manifestations, operationalizations or conceptualizations of the curriculum, then "curriculuming" (Boomer, 1982) or curriculum development should consist of changes in educational and organizational structure in the medical school. Therefore, during the process of curriculum reforms it is essential that medical schools invest sufficiently on creating the desirable learning environment.

In general, a qualitative shift occurs during studying when students gradually have to give up their original ways and become more active and self-regulated learners (Busato et al. 1998; Lindblom-Ylänne, 1999; Newble \& Clarke, 1986; Vermetten et al. 1999a). In medicine however, it is reasonable to state the problem has been that the traditional curriculum appears to promote quile a different line of development. Tooth, Tong, and Mcmanus (1989) have shown that students' approaches to studying develop more towards superficial and externally regulated methods during the medical studies. Hiliard (1995) reported that students at Toronto and other traditional Medical schools have higher surface learning scores or achievement learning scores and low deep learning scores than do students in PBL schools. However, there are also studies, which show evidence of an increase in medical students' scores on the deep approach later in the curriculum, even though they score more highly on the surface approach than students in an innovative medical school (Newble \& Clarke, 1987).

Among the studies conducted in medical schools, Newble \& Gordon (1985) confirmed that first year medical students show a similar pattern of scoring to science students more than arts students with respect to their learning approach. They also found that both third and six year students had significantly higher scores for meaning orientation than the first years and that there was no difference in reproducing orientation between the first years and sixth years. A cross sectional study using the $A S I$ concluded that there were no significant differences between the four groups of students from year one to seven on deep scores (Montecinos \& Pantoja, 1991).

It is commonly stated that medical education has relied too much on memorization and has placed too little emphasis on life long learning. In this regard, Hiliard (1995) compared the surface, deep and achieving scores of senior medical students in several cross sectional studies, using either $S P Q$ or Lancaster $A S I$, in a number of different medical schools. He reported that, in general, deep scores were the highest and achieving scores were the lowest among those students. One exception was a study conducted in a traditional school, where surface scores were the highest. Similarly, Newble and Clarke (1986) compared the scores of students in a traditional school and a problem based medical school (both groups being admitted on similar criteria) in Australia and showed marked differences in scoring. PBL students scored higher on deep approach and lower on surface approach than students in a traditional school. These facts strengthen the argument of some researchers that a traditional curriculum seems to guide students towards the adoption of less desirable study approaches (Coles, 1985; Lindblom-Ylänne, 1999; Newble \& Clarke, 1986; Steirnborg \& Bandaranayake, 1996; Tan \& Thanaja, 1993), while places where a problem based curriculum has been implemented have reported evidence of been able to guide students towards an elaborative, meaning directed learning (Jayawickramarajah, 1993). In a longitudinal design, Martenson (1986) also showed that the results from a conventional medical school (Karolinska Institute, Stokholm) compared well in many ways with a problem based school. Of course their curriculum had innova- 
tive features and was characterized by earlier clinical exposure, a higher degree of integration, and examinations measuring higher levels of knowledge. Lonka and LindblomYlänne (1996) reported that reproduction oriented and externally regulated learning was typical of novice medical students and was also found more frequently among advanced students. This does not seem to be limited to Finland; researchers from Australia and United Kingdom have also found that medical students do not show an increase in scores of a deep approach to learning during their studies (Newble \& Clarke, 1985; Steirnborg \& Bandaranayake, 1996; Tooth et al. 1989). In addition, epistemological development from dualist to relativist orientation was shown to be less common among Finnish medical students than among Finnish students in other disciplines (Lonka \& Lidblom-Ylänne, 1996).

Recently Dolmans and Wolfhagen (2004) compared the learning styles as measured by the $S P Q$ of medical students during a traditional and a problem based psychiatry appointment and found no difference. They concluded that individual students made differential use of the different attachments. Similarly Strømsø et al. (2004) studied changes in medical students' approaches to learning with the introduction of computer-supported problem-based learning at the university of Oslo and found no effect on participants' use of regulating strategies or their conceptions of learning.

\section{Student learning in higher education in Sri Lanka}

Among the limited studies carried out in Sri Lanka to understand the learning styles of undergraduate students, Perera (1998) attempted to validate the Sinhala version of Kolb's Learning Style Inventory, and reported that the learning styles of university undergraduates in Medicine, Engineering, Management, Science and Agriculture were significantly unequal in distribution and directed towards a converger style. He stated the results were similar to the results observed with westèrn students. Recently, Balasuriya, Dharmawardena, Rajapkse, and Jayasinghe (2000) conducted a cross sectional study on learning approaches and the performance at the university entrance examination of new entrant medical students selected to Universities of Colombo and Peradeniya in Sri Lanka, using a questionnaire developed by University of Western Australia. They concluded that the learning approaches i.e. achievement oriented and deep approach, were significantly associated with the performance at the entrance examination.

\section{Teacher centred versus student centred academic practice}

Vermunt (1996) pointed out, "instruction does not lead to learning automatically. The learning activities that students engage in during learning determine to a large extent the quality of the learning outcomes they achieve" (p. 25). However, teachers and instructional designers have a tendency to take over as many learning and thinking activities from students as possible. This view of teaching is being increasingly criticized and is founded on the idea that teaching essentially comes down to the transmission of knowledge from an external source to the learner (e.g. Biggs, 1996). On the other hand, Gow and Kember (1993) suggested that the methods of teaching adopted, the learning tasks set, the assessment demands made and the workload specified are strongly influenced by the orientation to teaching. They further reported that in departments where a knowledge transmission orientation predominates, the curriculum design and teaching methods were likely to have undesirable effects on learning approaches of students. Departments with a greater propensity toward learning facilitation were more likely to design courses and provide a learning environment encouraging meaningful learning. 
Brown, Collins and Duguid (1989) observed that teaching often leads to isolated and inert knowledge. Inertness of knowledge refers to the problem that is often seen in working practice e.g. the problem of inability to apply the acquired knowledge to solve problems in actual practice. Dahlgren and Marton (1978) showed that although university students were able to talk about their field of study in more complicated words after one year of studies, their misconceptions about fundamental phenomena in that field had not changed. This traditional type of teaching does not foster the development of students self-regulatory skills (Boekaerts, 1997; Vermunt \& Verloop, 1999). However, it is not uncommon to find teachers who believe students learn and develop through exposure, and that the content is all-important. Having been accustomed to a traditional learning process where one that knows (the teacher) presents ideas to one who does not know (the student), it is not surprising that teacher's hold this attitude. Another characteristic of the traditional process is the emphasis laid on coverage of material through teaching by telling e.g. lectures.

Although the above approach may work for the teachers, it may not work for the majority of today's students. Besides gaining an understanding of the course content, which may be the teacher's main aim, students mention the personal affective aspects of study such as gaining confidence, changing attitudes and increasing critical awareness and scepticism (Beaty et al., 1997). Recently, Trigwell and Prosser (2004) provided empirical evidence that teacher focused approaches to teaching were associated with students' reproducing orientations. They went on to state that in situations where teachers adopted more student-focused approaches to teaching, their students adopted a deeper approach to learning. Boekaerts (1997) found that encouraging students to accept greater responsibility for controlling their studying rather than relying on teachers to direct their learning resulted in more effective learning. There appears an urgent need to bring about a change in teaching. Since students can learn how to learn by practicing how to do it, they should be provided with opportunities to learn the skills of self-regulated learning.

\section{Process-oriented teaching}

In contemporary conceptions of teaching, a central place is given to the quality of student learning (Vermunt \& Verschaffel, 2000). The aim is to develop skill in the use of learning and thinking activities that the learner is not inclined to use. Learning to learn has increasingly become a major goal in education. This calls for teaching theories and instructional design models that are specifically aimed at promoting learning-to-learn processes in students. From an epistemological point of view, an important objective of instructional measures will be to facilitate active knowledge construction among learners. From a societal point of view, it is important that education consciously promotes the ability of students to self initiate such types of learning. Process oriented teaching is based on the interplay between student regulation and external-regulation (teacher or instructional agents) of learning processes. The characteristic feature of process-oriented teaching is the gradual and systematic transfer of control over learning processes from instructional agents to learners (Vermunt, 1996; Vermunt \& Verschaffel, 2000). Similar to learning activities teaching functions too can be divided into processing (presenting and clarifying subject matter), affective (creating and maintaining a positive motivational and emotional climate) and regulation (steering students learning process) components (Vermunt \& Verloop, 1999). These teaching functions are increasingly used in student oriented learning environments as a means to steer the process of constructive learning In essence, the primary objective of process oriented instruction is to: (1) develop the use of desirable 
metacognitive strategies by learners, and (2) transfer the responsibility for learning from an expert to the novice through the activities of modelling, coaching, fading and reflective learning (Volet, McGill, \& Pears, 1995).

Students selected for university have already acquired some skill in employing learning strategies, but they have a need for further skill development. Therefore, a shared control strategy is best suited for these academic contexts where by challenging students to try new forms of learning or with teacher guidance, students are made to engage in learning activities that they are not used to doing so far. In this instructional model teachers for the most part initiate and support the thinking activities that students employ in their learning (Simons, 1997).

Very recently, Ten Cate, Snell, Mann, and Vermunt (2004) proposed a process oriented teaching model, which they called "Learning oriented Teaching model" (LOT). This model describes the transition from external guidance (teacher playing a dominant role in regulating the learning content, learning activities and the goal setting) through shared teacher guidance to internal guidance (student on his own decides on what to study, what books to read, what skills to perfect as relevant to the individual's goals). They described how the different stages of this LOT model could be realized within an existing problem based curriculum in Dutch and Canadian medical schools.

\section{Problem based learning}

Problem based learning is increasingly referred to as an important development in health profession education in the latter part of the twentieth century. Howard Barrows at McMaster University in Canada (Barrows \& Tamblyn, 1976) first developed this approach. Since then new medical schools throughout the world have adopted PBL as the educational and philosophical basis of their curricula and some traditional schools have incorporated this concept in the teaching learning process to varying degrees. Some others have very boldly converted their undergraduate programmes to PBL (Davis \& Harden, 1999). The General Medical Council (GMC) has advocated a problem-oriented approach in its recommendations for basic Medical Education (General Medical Council, 1993). Medical schools are well aware of the merits of the learner centred and problem based approach in improving the cognitive skills of learners in controlling, planning, monitoring, regulating and evaluating their learning processes.

Problem based learning is thought to have reversed the traditional approach to teaching and learning. It encompasses the constructivist notion of learning and the active role of the learner in the learning process. Therefore the role of teacher in PBL is very different to the role of a teacher in a traditional curriculum. According to Albanese and Mitchell (1993) "... PBL at its most fundamental level is an instructional method characterized by the use of patient problems as a context for students to learn problem solving skills and acquire knowledge about the basic and clinical sciences" (p. 53). Therefore, PBL could be considered as yet another instructional method, which can be included in the teacher's tool kit along with other teaching methods. As described by Boud (1985), the principle idea behind PBL is that the starting point for learning should be a query or a puzzle that the learner wishes to solve. Barrows (1998) indicated that PBL is explicitly used to get students to think. Unlike in the conventional method, in PBL the learning process is initiated and guided by a sequence of problem tasks. Subject matter is not presented to students directly but covered by tasks (which could be cases, research papers, video clips) that require working in small group settings supported by a tutor. In these small groups students organize their ideas and previous knowledge related to the problem 
that they do not understand. This thinking aloud process promotes the activation of relevant pre-knowledge and makes students aware of uncertainties and gaps in their knowledge base. In the PBL approach students analyze the problem that is extracted from the task description, discuss relevant aspects of the problem and hypothesize about possible conceptualizations and solutions. Thus, within PBL students acquire life long learning skills, which include ability to find and use appropriate learning resources (Duch, 1995).

Since learning occurs in a small group context, which is a type of "cooperative learning", this approach seems to improve students' social skills. According to Johnson and Johnson (1994), cooperation "... is the heart of interpersonal relationship, families, economic system and legal system ... Understanding the nature of interdependent systems and how to operate effectively within them is an essential qualification of future citizens" (p. 15). In contrast to the traditional instructional approach where students passively receive knowledge provided by the teacher, in cooperative learning students are given equal chances in the distribution of power and knowledge. Students are allowed as well as encouraged to freely express their ideas and feelings. Moreover, students share the responsibility of establishing an understanding of tasks and strategies. This will ultimately lead to the creation of a student who is more positive about him or her, more active and critical in receiving information.

Numerous studies have examined the process and outcomes of PBL in a variety of cognitive and non-cognitive areas (Albanese \& Mitchell, 1993; Jayawickramarajah, 1995; Kaufman \& Mann, 1997; Norman \& Schmidt, 1992; Vernon \& Blake, 1993). Norman and Schmidt (1992) reviewed the experimental evidence supporting possible differences in students' learning that can be attributed to PBL and found among other things that PBL enhances intrinsic interest in the content to be mastered and also enhances and maintains self-directed learning skills. Albanese and Mitchell (1993) noted that compared to conventional instruction, PBL is more nurturing and is enjoyed by both students and teachers. Vernon and Blake (1993) following a meta-analysis concluded that the comparative value of PBL curricula over conventional curricula is greater in the areas of students' program evaluations, staff attitudes, student mood, class attendance, academic process variables and measures of humanism. Jayawickramarajah (1995) indicated that empirical evidence derived from qualitative as well as quantitative methods have shown that the PBL strategy has been more effective in satisfying educational needs of learners and the graduates of these PBL schools are well equipped to confront health care problems in practice. Thus evaluative studies on problem based learning in medical education reveal its superiority over more traditional methods.

Comparisons between medical students' approaches to studying in two different types of medical schools, traditional and innovative, have shown that those in an innovative school scored highly on the deep approach, whereas those in a traditional school scored highly on surface approach (Newble \& Clarke, 1987; Schmidt, Dauphinee, \& Patel, 1987). Problem based learning was shown to be associated with low surface and high deep and achieving approaches, a pattern which increasingly sharpens from first to final years (Newble \& Clarke, 1986).

\section{Implications for curriculum design}

Increasingly the curriculum is seen as covering not only what is taught but also how it is taught and learned, how the learning is managed and how the overall learning environment is designed (Harden, 2001). While looking for a simplistic yet comprehensive definition for a curriculum, Gemn (2001) has adopted the following from Stenhouse (1975, 
p.2): "everything that is happening in the classroom, department, medical school or the university as a whole." Thus, the environment of the medical school is thought to be a manifestation of its curriculum (Genn, 2001).

Going back to the argument that expert knowledge cannot be transmitted, then the individual must construct it. It is very important that learners are taught to employ suitable learning and thinking activities to construct, change and utilize their knowledge. Curriculum design should be based on the philosophy 'less is more', that is on the idea that learning a few important ideas and concepts well is educationally much more powerful than learning an extensive amount of superficial facts (Resnick \& Collins, 1996). Recent research on variation in student learning offers a sound basis for instructional and environmental reform in higher education. Therefore, curriculum development in medical schools should consist of changes in educational and organizational environments. This is in line with Genn's (2001) conceptualization of the Medical school as a learning organization.

Those who are interested in bringing about educational reforms need to pay attention to the organizational arrangement. In this respect Ramsden (1988) and Biggs (1993) described several nested systems relevant to student learning; i.e. (a) the student system comprising equilibrium between cognitive and affective factors, and perceived phenomena, (b) the classroom system, comprising students, teachers and teaching context, the institutional system, which itself contains subsystems at department and faculty level (each of them having the potential for enhancing or, as Reid (1987) considers more likely, impeding enlightened practice) and (c) the community system, which has recently in many countries imposed its own constraints on higher education, that have effects on the classroom level. Each subsystem attempts a steady state of equilibrium internally between its own components, as well as with its super-ordinate system. This fact is critical for understanding whether intervention is likely to effect change, either at student level with study skill training or in staff development at the classroom level or structural innovations at the level of the institution itself.

\section{The role of culture in shaping educational and learning practices}

\section{Dimensions of a national culture}

Culture dependent differences in thinking and acting and its implications for education are extensively discussed in the literature. Interestingly, Hofstede (2001) identified five dimensions of culture. They are: (1) the extent to which the less powerful members of a society accept and expect that power is distributed unequally; (2) the extent to which the members of a society feel comfortable or uncomfortable in unstructured situations; (3) the degree to which individuals are supposed to look after themselves or remain integrated into groups; (4) dominant gender role patterns in the society i.e. the degree of expression of "toughness" among males and "tenderness" among females in handling situations; and (5) the extent to which a culture programs its members to accept delayed gratification of their material, social and emotional needs. He has classified different countries on the different dimensions of culture and described the possible influences it could have on the education system. Cross-cultural researchers can make use of these dimensions to assess the differences and similarities of the cultures being investigated. 


\section{Cross-cultural research on student learning}

Education and culture are so interrelated that it is hard to discern effects of culture on education. However, researchers have shown a great interest in investigating the effects of culture on student learning. Watkins and Regmi (1992) interviewed Nepalese students about their conceptions on learning and found no evidence for the conception of learning as memorising and reproducing that earlier research had found among European students. Tang (1991) interviewed Hong Kong students and found two categories of memorization, which she described as surface and deep memorization. Surface memorization corresponded with surface approach while the students who desired to use a deep approach adopted a deep memorization approach when they found that their courses, the assessment of them, stressed reproduction. To obtain a good mark it was found to be necessary to commit bodies of information to memory. Hence these students employed a combination of understanding and memorizing in order to succeed. "You have to memorize for a test but you still need to understand first. If you try to rote memorize you soon forget... Rote memory cannot be that long term, while a memory with understanding can be a long term one "(a literary quote cited by Kember, 1996 from Tang, 1991).

Since it is reported that Chinese and Japanese believe that understanding may come through memorization (Marton et al. 1997; Purdie, Hattie, \& Douglas, 1996), memorisation is used as an aid to understanding. Thus, it is not justified to equate that with rote learning. Kember and Gow (1990) reported another distinct entity as a "narrow orientation" in which students displayed an intention to understand and memorize. Hess and Azuma (1991), having analyzed the contents of interviews with a group of Japanese students, documented evidence for understanding being gained through repetition and memorization in Japanese schools. They noted that in Japan, students are encouraged to learn from traditional Confucian wisdom, which advocates, "read it one hundred times and understanding will follow spontaneously". However, Chinese students with advancing age seem to employ repetition and variation simultaneously as a means to deepen understanding and remember better. Recently Marton, Weng, and Wong, (2005) reported that Chinese university students spoke about memorisation and understanding as simultaneous events, two different aspects of the learning process. They disclosed the fact mature students repeat different presentations of the same thing or read the same presentation in different ways thus, use repetition and variation at the same time.

In describing the 'Asian paradox', Kember (1996) indicated that despite apparent attempts to memorize, Asian learners have proven to be high achievers. The literature also reveals that Chinese and Japanese believe that understanding may be gained through memorization (Marton et al., 1997; Purdie et al., 1996). Memorisation is used as an aid to understanding thus; it is not proper to equate memorization to 'rote learning'.

Kember (1996) summarised the findings of interview research with Chinese students and teacher educators and was of the view that the dividing line for these students does not fall between memorization and understanding but between mechanical memorization and memorization to assist development of meaning. Hence, the possibility of existence of subcategories of memorization with understanding was postulated; one being understanding, preceding memorization and the other being memorization, preceding understanding (Kember \& Gow, 1990; Marton, Dall'Alba, \& Tse, 1992).

Richardson (1994) also noted that the orientation towards comprehension was consistent between countries, probably because of the concordance of the espoused aims of higher education between systems. Nevertheless, less desirable approaches appear more variable and less coherent possibly because it is usually adopted as a consequence of stu- 
dents' perception that the learning environment is unsatisfactory. Similarly, Kember and Gow (1990) concluded that there were cultural influences on less desirable study approaches and speculated limited command of the language and the nature of schooling and upbringing as factors leading towards a narrow approach.

Volet, Renshaw, and Tietzel (1994) investigated the cross cultural differences in study approaches among Australian and Southeast Asian first- year students, following the same academic course in an Australian University, using Bigg's (1987) study process questionnaire. They found that there were significant differences between the two groups only on two of the subscales at the commencement of the semester. On the one hand, Southeast Asian students scored lower than local students on the use of a deep strategy while they scored higher on a measure of students' tendency to accept ideas of lecturers at the beginning of the academic course. When factor loadings of the $S P Q$ items were studied Volet et al. (1994) also noted some inconsistencies in factor loadings of Asian students as compared to those of local students, However, they found that at the end of one semester after studying in the same context, the patterns of change in Asian students were similar in nature and direction to those of Australian students. This study highlighted the impact of contextual influences on students' study approaches and queried the role of stable individual characteristics or cultural differences in determining the study approaches. Volet et al. (1994) also documented the higher achieving nature of Southeast Asian students. It is in line with Bigg's argument that Asian students place particularly high value on academic achievement. Therefore, it is reasonable to assume that this behaviour is partly related to their cultural practice of attributing successes and failures to effort.

Kember and Gow (1991), using the $S P Q$ with Australian and Hong Kong students attending a variety of degree programs, found that Hong Kong tertiary students scored higher on deep approach scales and lower on surface approach scales. Both studies stress the importance of the different teaching practices across cultures in shaping students approaches to study. They further argued that like those of western students Hong Kong students are responsive to the demands of the academic context and that the anecdotal claim that Asian students are inherently more inclined to be rote learners is not supported by serious empirical evidence.

Watkins, Regmi, and Astilla (1991) compared 14-16 year old Filipino and Nepalese students answers to those of Australian and Hong Kong students using $S P Q$. The factor structures they obtained were highly similar. Nepalese students reported higher levels of both deep and achieving approaches to learning than the other students. Watkins and Akande (1994) obtained a similar factor structure with $S P Q$ with Nigerian students. Remarkably similar solutions have been obtained in cultures as diverse as Britain, Nepal and Nigeria, although not in the Philippines (Richardson, 1994).

Purdie et al. (1996) examined differences between Australian and Japanese secondary school students' conceptions of learning and their use of self-regulated learning strategies. They noted that the Japanese students view learning from a much broader perspective whereas Australian students have a more 'narrow' school- based view of learning. A conception of learning as "understanding" is associated with greater total use of strategies for both Australian and Japanese students.

Marton et al. (1997) interviewed Chinese high school students about their conceptions and experiences of learning. They found that these students did not consider memorization and understanding as opposites as Western students often did. Ajisuksmo and Vermunt (1999) compared the factor structures of Dutch and Indonesian students using the $I L S$ and the $I C B$ respectively and reported among other things, that Dutch students 
experience aspects of learning patterns as separate that for Indonesian students can go well together.

Previous research with American students has found that high achievers make great use of certain self-regulated learning strategies. These achieving students have also demonstrated a greater reliance on social sources of assistance, peers, teachers and other adults (older sibs). However, Purdie et al. (1996) reported that although the Japanese students were also found to be high achieving they sought significantly less assistance than did the Australian and was explained by the cultural emphasis on personal effort among the Japanese as a major source of achievement.

Very recently, Van Mil (2005) compared the learning strategies, conceptions of learning and learning orientations of Dutch and Chinese students following the same Bachelor of Science program at Wageningen university in the Netherlands and found that the latter indicated the use of repetition and memorization and stepwise processing strategies more often, and also that they were test-oriented. However, the Chinese students scored higher on self-regulation strategies and construction of knowledge. Mil also found that despite hard work the academic performances of Chinese students were disappointing.

Cross-cultural research suggests that cultural factors influence the cognitive processes used by students (Ajisuksmo \& Vermunt, 1999). Qualitative research conducted in many countries, Western as well as Asian, shows that to a reasonable extent cultures differ in the emphasis they place on encouraging constructive learning in students and making learning events more appealing to students (Hess \& Azuma, 1991; Hofstede, 2001). Based on the evidence of their longitudinal study of socialization and education in Japan and the United states, Hess and Azuma (1991) indicated that adults in Japan, as opposed to those in USA, are particularly eager to prepare their child to be diligent and cooperative with the teacher. Moreover, mothers and children in Japan tended to see the causes of performance as internal, whereas their counterparts in the USA often included sources beyond the control of the child.

Two of the most commonly cited behaviours shared by many Asians (Japanese, Chinese, Thais, Malaysians and Indonesians) seem to relate to memorization and the relationship between student and teacher (Purdie et al. 1996). Asian cultures, in general, tend to replace the role pair parent-child by the role pair teacher-student along with the basic values and behaviour from one sphere to the other (Hofstede, 2001) Thus, the average student expects teachers to tell them everything that they know, exhibiting a high degree of academic dependence, and believes that it is not correct to challenge the views of teachers out of respect.

Hofstede (2001) referred to a 'Confucian Work Dynamism' that was identified by the Chinese Value Survey (Bond, 1988), being composed of values such as persistence and perseverance, ordering relationships by status and observing this order and having a sense of shame. He further reported that the Asian countries, e.g. China, Japan, Malaysia and Indonesia as well as India (though a non-Confucian country), uphold these values unlike their Western counterparts. These values could be making a substantial contribution towards their high achievement. Some studies show that Australian students are stereotypically viewed as more active in their approaches to learning than Asian students. Similar to American students, Australian students too are characterized by assertiveness, independence, self confidence, acceptance of diversity and willingness to question and explore alternative ways of thinking and acting (Purdie et al., 1996). On the other hand, task persistence and attribution of failure and successes to lack of effort more than lack of 
Chapter 3

ability among Asians is speculated to be a factor contributing to their high achievement. Thus, researchers sometimes explain exceptional findings in cross-cultural investigations as a consequence of its cultural context (Thomas, 1998). Differences in learning patterns among students from different countries are increasingly viewed as a consequence of cultural milieus (Ajisuksmo \& Vermunt, 1999). Therefore, it is prudent to consider consequences of culture in drawing up inferences about student learning behaviours in any reform process.

\section{Conclusions}

An upsurge in the interest of assessing study strategies was witnessed in the last decade. Learning strategies of university students and their developments have been the focus of many studies. Some of these researchers have utilized a range of learning inventories in order to assess the learning variables quantitatively. Among the available inventories, the $I L S$ with its strong focus on regulation of learning and collaborative aspects of learning is considered an appropriate research tool to assess the degree of self-regulation of the learning process. Many researchers have used the $I L S$ or one of its adapted versions to measure the learning strategies, conceptions of learning and learning orientations in a wide range of disciplines, in both Western and Asian countries.

As indicated in this chapter, researchers have repeatedly shown that various factors in the learning environment, (particularly the type of instruction, curriculum philosophy and examinations) and in the students themselves (prior knowledge, personality, attitudes to courses) affect the way students go about their learning and studying. Hofstede (2001) pointed to the interrelationship between culture and education drawing attention to the study of culture specific learning patterns among higher education students.

Although many researchers have investigated learning patterns of university students in different contexts, the learning patterns of Sri Lankan medical students at the university of Peradeniya have not been the focus of research in the past. Given the potential of the outcomes of investigating the learning patterns, changes in reported study strategies in longitudinal and cross sectional designs, and student perceptions of learning context in education decision making, a systematic study of those aspects within the specific institutional context was deemed to be a useful exercise. The fact that learning is an extremely complex phenomenon calls for psychological theories derived from the settings to which they are to be applied. Therefore, it is important to study aspects of learning in natural environments (ecologically valid) rather than in a laboratory. The outcomes of such an investigation apart from setting direction for curriculum reforms could also clarify current beliefs about learning in higher education. 


\section{Introduction to the empirical studies}

\section{Background}

In the recent past, an increased interest in describing and measuring study strategies of students in higher education was witnessed. This practice gained momentum due to the fact that all over the world convincing empirical evidence is increasingly being sought to inform policy makers and teacher trainers for effective decision-making. As was pointed out by Entwistle and McCunne (2004), this trend is partly a reflection of the higher education institutes becoming more socially accountable.

It is accepted that learning is a complex phenomenon. Personal as well as environmental factors play an important role in producing desirable learning outcomes. In addition the home and the school environment could play an important role in shaping individual learning behaviour. A large number of research studies conducted in the past have investigated the associations between the quality of learning and the learning environment. In this respect, several educational researchers have shown that the learning environment (teaching, learning activities and assessment procedures adopted by an institution) plays a crucial role in the development of qualitatively better learning (Bloomfield, Harris, \& Hughes, 2003; Fransson, 1977; Nhan, 2006; Ramsden, 1997; Ramsden \& Entwistle, 1981; Trigwell \& Prosser, 1991; Trigwell, Prosser, \& Waterhouse, 1999; Vermetten, Vermunt, \& Lodewijks, 1999a; Vermunt, 2005). However, longitudinal studies examining student learning patterns in a South Asian medical school context are scarce.

\section{The Sri Lankan study}

Although the necessity to move towards student centred education is discussed repeatedly during the curriculum committee meetings, the staff of the Faculty of Medicine University of Peradeniya (FMUP) does not appear to be convinced as to why one should change. "Can't we achieve the same outcomes with the present system?" is a question that is often posed. Is it merely for the sake of changing that we want to change or is it imperative to improve the quality of learning? Have we assessed the learning activities employed by our medical students and shown that they are not desirable? It had been a daunting task to answer the questions stated above in the absence of empirical evidence. Hence, exploration of learning patterns of medical students was identified as a priority area for educational research.

An important aim of tertiary education is that students develop more advanced, deep level learning. Thus, it is expected that novices will develop academically more desirable learning strategies and attitudes as they advance in their studies. Furthermore, development of expertise in professional education involves the process of developing domain specific competence, epistemological development and a shift towards more self regulated and meaning -directed learning (Lindblom-Ylänne, 1999). It has also been made clear that learning functions play a central role in the theory on regulation of learning processes (Vermunt, 1996, 1998). However, the manner in which undergraduate medical students in the Sri Lankan educational context carry out these functions, and the way in which this execution of learning strategies is regulated by internal and external sources, has not been studied. Insight into these processes can make an important contribution to the improvement of instructional practice in medical education. In fact, that is in line with 
the foregoing argument that learning and thinking activities of students should be taken as a starting point in designing instruction. Thus, exploring the "learning styles" or "learning patterns" of medical students was considered to be useful for educational decision making and counselling, especially at a time when there is much discussion about higher educational reforms. Towards this end, a research project has been designed to study aspects of medical students' learning within the existing medical curriculum at the Faculty of Medicine Peradeniya. The construct of learning style or learning pattern used in this thesis is a coordinated concept and refers to a coherent whole of the learning strategies, learning orientations and conceptions of learning which characterize each student's learning in a certain period of time (Vermunt, 1998).

Against this background an attempt was made to investigate the trends in the development of learning strategies and self-regulation among a group of Sri Lankan medical students within the context of a traditional curriculum. The overall aim of the research project was to explore the puzzle of student learning within an Asian medical school context. Thus, this project comprised a series of empirical studies to address the following main research questions:

1. What are Sri Lankan medical students' study strategies, conceptions of learning, values, beliefs, attribution patterns, expectations of university teaching and learning and study motives?

2. Can the ILS be adapted to form a valid and reliable diagnostic instrument?

3. What are the learning patterns of Sri Lankan medical students?

4. What are the associations between students' use of learning strategies, their learning conceptions and orientations on the one hand and contextual factors (such as parental education and occupation, and district from which the student secured university entry), personal factors (such as gender, attempt at which university admission was secured, independent study time), and academic performance on the other?

5. Does the national culture play a major role in explaining the students' study strategies and the interrelations between the different aspects of learning?

6. What are the trends in the development of learning strategies?

7. What are the students' perceptions of the existing learning environment?

Additionally the project was expected to broaden the understanding of the learning process, which included the investigation of consistency and variability of learning strategies across time and to make a cross cultural comparison of the learning patterns distinguished by the ILS, ICB and the ARPM. This all may provide a wealth of information and help in the educational decision-making and staff development programmes.

\section{A conceptual model underlying this dissertation}

Figure 4.1 depicts a model of student learning in higher education, containing the basic concepts and their interrelations central to this dissertation. The purpose of this simplified model is to visualize the theoretical basis of the thesis. The part to the left of the perceptions "box" of the model represents the personological domain of learning, which includes the student characteristics and learning processes. In this part, the role of the person or the object of the educational process is displayed. In the right part of the model, situational influences are represented under the heading learning environment. In addition to the immediate learning environment, variables such as the home environment (parental education and occupation), the secondary school environment, and the attempt at which 
university entrance was secured were hypothesized to have an impact on the learning behaviour and were included in the periphery. The middle part of Figure 4.1, connecting the two, depicts the interaction between the learner and the environment. The greater part of the model is adapted from Vermetten (1999), which in turn is based on Ramsden's (1988) model of situational influences on student learning and Entwistle's (1991) view that "... it is the student's perception of the learning environment that influences how a student learns, not necessarily the context itself" (p.202). The centrality of the learner, which is consistent with the constructivist view, is very much in focus in this model. The part to the extreme left is adopted from Hofstede (2001), where he described the influence of different aspects of culture on education and organizational arrangement (institutional arrangement for example, the hierarchical arrangement which impedes innovations).

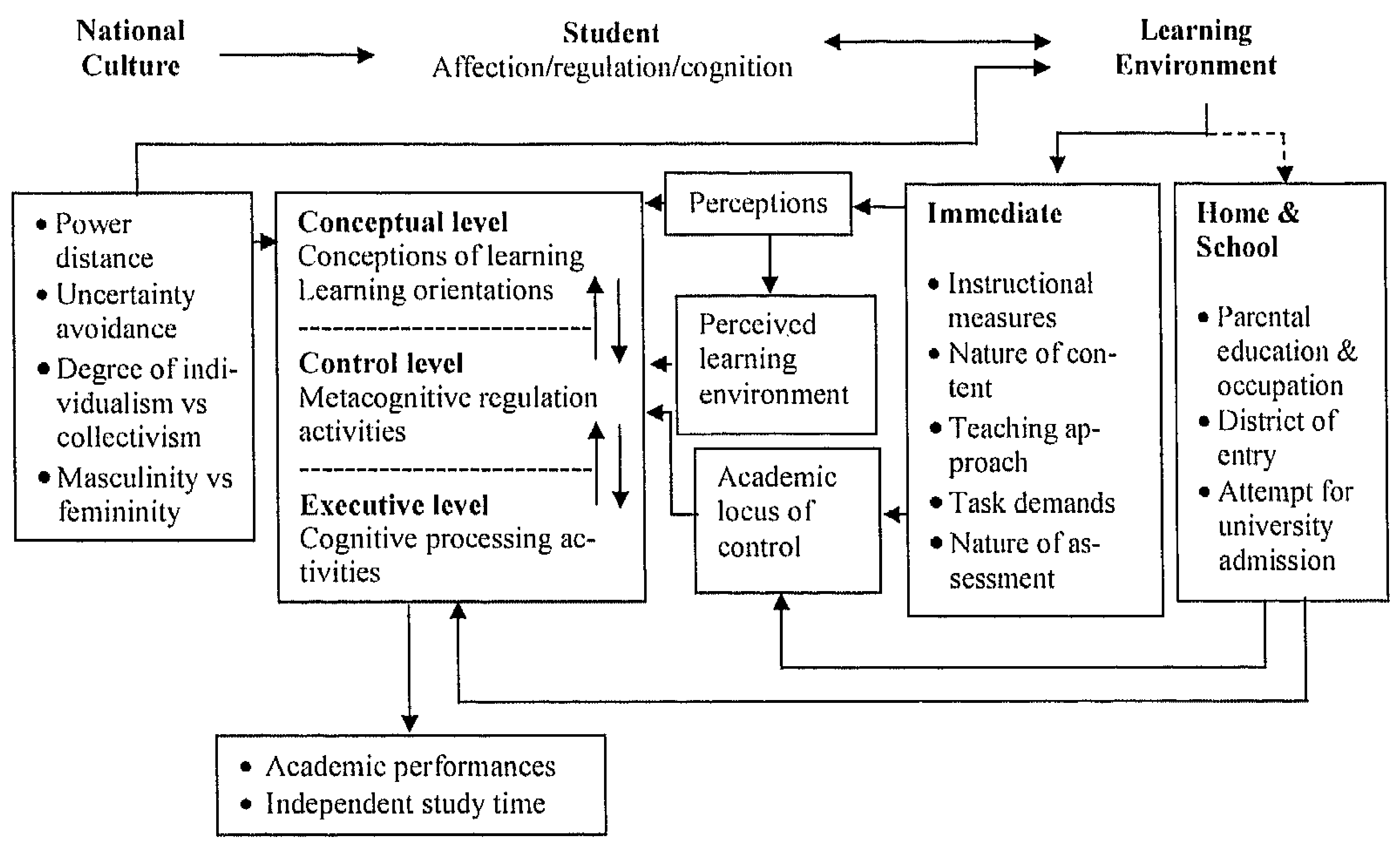

Figure 4.1. Student learning in higher education - a conceptual model underlying the dissertation.

In the left part of the model the three types of learning activities discussed in chapter 3 are shown. They operate at three levels: the conceptual level, the control level and the executive level. The four components of Vermunt's learning theory (1998), which also include the constituents of the ILS, are placed on these three behavioural levels. At the highest level conceptions of learning and learning orientations are located, according to Vermunt (1998) these are relatively static aspects of learning. At the middle level metacognitive regulation is found. The lowest level contains the cognitive processing activities, which according to Vermunt's learning theory are the least stable. In addition to these behavioural levels, a taxonomy of individual differences (Snow, Corno, \& Jackson, 1996) consisting of personality traits, values, attitudes, beliefs and goals could be added to the student part of the model. However, that is not included in the model shown below as that is beyond the scope of the present study. Nevertheless, it is acknowledged that the cultural dimensions have a strong influence on those characteristics, values, beliefs, goals, etc. 
The model further portrays the possible associations between academic performance, independent study time and the reported use of study strategies.

To the extreme left of Figure 4.1, the cultural dimensions that have an impact on learning behaviours of students and teachers are enumerated. Similarly, to the right of the perception box the important environmental elements are presented, some of which correspond to the three related contextual domains described by Ramsden (1988).

According to the model individual perceptions are formed by the interaction of student conceptions and contextual factors. The results of the perception processes are represented as the perceived learning environment. As shown in the model, it is believed that the control between the perceived learning environment, the students' conception of learning, learning orientations and other relevant individual characteristics (though not included in the model) occur through the meta-cognitive regulation activities. The cognitive processing activities are mainly 'driven' by these regulation processes.

Reciprocal arrows drawn between behavioural levels indicate that information flows are believed to go up and down both (like feed forward and feedback). The double arrow at the top indicates that there is two-way interaction between student and learning environment. National culture has its greatest impact on the student; however its effects are also manifest in institutional culture and nurturing. An attempt was made to include the effects of home environment and schooling on learning behaviour. As depicted by the model, the nurturing environment influences the development of academic locus of control. However, the immediate learning environment could also be an influential factor. Thus, academic locus of control could be considered as a function of the student and the learning environment, which in turn regulate the use of cognitive processing strategies and regulation strategies by the student concerned. The arrow from the culture to student behaviour depicts the powerful influence it could have on the individual (although not indicated in the model this effect is through the personality traits, beliefs values etc.). However, the centrality of the learner in the educational enterprise is well shown by this model. It must be emphasized that this model is not meant to be an ideal representation of the 'real' and complex processes of learning in higher education. Nevertheless, it will clarify some of the theoretical notions presented in the introductory chapter.

\section{Research context}

The research project was implemented from August 1999 to September 2001 at the Faculty of Medicine, University of Peradeniya, Sri Lanka. The participants were: (1) new entrant students, (2) third year and (3) fourth year undergraduate medical students of the Faculty of Medicine Peradeniya.

The undergraduate curriculum at the faculty of Medicine, Peradeniya, can be described as a discipline based traditional curriculum (see chapter 2). The medium of instruction is English. Up to senior secondary education the medium of instruction is Swabasha; in the professional undergraduate courses it changes to English. The duration of the MBBS course is five years. This course comprises three distinct stages: a) the preclinical course- first four terms of work where the basic sciences are taught; b) the third MBB part I and part II courses - six terms of Para clinical work; and c) the final MBBS course, one year of mainly clinical work. The selection of students to Sri Lankan medical schools is purely on merit as described in chapter 2 . The teaching programme is more of a teacher centred didactic one with great emphasis on terminal assessments. Students 
spend relatively many contact hours with teachers in either lecture sessions or tutorials. Furthermore, students are provided with objectives, introductions, summaries, rehearsal units, tasks, tests with feed back, and study materials compiled by teachers during the course.

\section{Empirical Study 1 described in chapter 5}

First and foremost it was essential to develop a culturally acceptable measuring tool to obtain data on students' learning activities, learning orientations and conceptions of learning. In order to accomplish the above task a qualitative analysis of the beliefs and views of new entrant medical students on learning, learning orientations and learning strategies was required.

As a first step it was felt prudent to conduct semi-structured interviews in the students' mother tongue language to empathetically understand their approaches to learning, views on learning and studying, and their study motives. The interviews were conducted among a randomly selected group of new entrant medical students who were attending the English course prior to the commencement of the academic course.

The main objective of the above research approach was to discern different categories of description for the four main components of learning described by Vermunt (1998): cognitive processing activities, regulation activities, study motives and conceptions of learning as well as factors which facilitate learning and attribution pattern among Sri Lankan students.

The second objective was to become familiar with aspects of learning and terminology described by these students and to include aspects that may be missing in the parent inventory (ILS, see chapter 3 ). The results of these interviews were helpful in developing the adapted Sinhalese version of the ILS, which is called the Adyayna Rata Prakasha Malawa.

The interview study described in chapter 5 attempted to answer the following research questions:

1. What are the attributes of "an Ideal Student" from the perspective of Sri Lankan entrant medical students?

2. What are factors that have facilitated learning so far among these novice medical students?

3. Which cognitive processing activities did these students employ during their senior secondary education?

4. To what degree do students report internal and external control of the learning processes at the commencement of the undergraduate studies?

5. What kind of conceptions of learning and teaching do they report at the commencement of university education?

6. How do entrant Sri Lankan students attribute academic success and failure?

7. What are their expectations of learning and teaching in the university?

8. What motives underlie their selection of the medicine course?

9. Do the students' descriptions of the components of actual learning (cognitive processing, regulation, conceptions of learning) and expected learning show any associations? 


\section{Study 2 described in chapter 6}

The development of Adyayana Rata Prakasha Malawa (ARPM) and its validation was the main focus of this quantitative study. The Inventory of Learning Styles (Vermunt, 1998) was developed in the Netherlands (in a European culture), therefore the objectives of study 2 were to: 1) adapt the inventory to Sri Lankan students, develop a Sri Lankan version of the ILS, which is called ARPM and validate it, 2) to get an overview of the learning patterns employed by Peradeniya Medical students.

The study objectives were translated into several research questions:

1. What is the reliability of the ARPM-1 scales?

2. To what extent can the internal consistencies of scales be increased?

3. Which learning styles of Sri Lankan students can be identified by the ARPM-2?

4. What is the relationship between students' ARPM scale scores and personal factors such as gender, attempt at entry and average amount of independent study time per day?

5. What is the relationship between students' ARPM scale scores and contextual variables such as level of maternal education, parental occupation, district from which the student has secured entry to medical faculty?

6. What is the association between students' ARPM scale scores and academic performance?

7. Is there a relationship between the ARPM scale scores and students' academic locus of control? Does the academic locus of control change among the different student groups?

The ARPM was administered to obtain data on different learning aspects i.e. learning strategies, learning orientations and conceptions of learning, while a separate data form was constructed to collect the personal data necessary to study the associations between variables under study ( i.e. attempt at which university admission secured, district of entry, parental education level, parental occupation, and the attributions during academic failure).

\section{Study 3 described in chapter 7}

The Inventory of Learning Styles (ILS), the Inventarisasi Cara Belajar (ICB) and the ARPM belong to a family of instruments. These three inventories have been used to assess learning styles of first year university students in three different cultural contexts. It is reported that the ILS was used in a number of western countries while ICB has been used in Indonesia and ARPM in Sri Lanka. Thus, a cross-cultural comparison of the results of the ILS and its adapted versions was attempted. The objective was to compare the learning styles identified among medical students with those obtained with the family of inventories among the Dutch students and the Indonesian students on similarities and differences, or more specifically whether an Asian learning style could be identified.

This study addressed the following research questions:

1. Do Sri Lankan students differ from Dutch and Indonesian students in terms of the reported use of study strategies, orientations of learning and conceptions of learning as assessed by the ARPM, ILS and ICB respectively? 
2. Are there similarities and differences in the factor structure of learning patterns identified by the ILS, ICB and the ARPM among first year Dutch, Indonesian and Sri Lankan University students?

3. Are learning patterns discerned within the first year of university education theoretically congruent among the first year Sri Lankan medical students and the first year Dutch and Indonesian students?

The results of the three studies were analyzed and discussed in a cross-cultural perspective.

\section{Study 4 - described in chapter 8}

This study investigated the trends in the development of learning strategies among Sri Lankan medical students as they progressed through the present, traditional curriculum and their perceptions of the learning environment. Objectives were to: (1) identify the trends in the development of learning strategies in a cross sectional design and a longitudinal design within the context of a traditional curriculum; and (2) identify the environmental factors that may be contributing towards the observed changes in aspects of learning.

The research questions were:

1. Do learning strategies, conceptions of learning and learning orientations differ between the three groups of students who differ in the study phase they are in, i.e. new entrants, $3^{\text {rd }}$ year and $4^{\text {th }}$ fourth year medical students?

2. Do reported learning strategies, conceptions of learning and learning orientations differ within two groups of students at two moments in time with an interval of 30 weeks and 50 weeks?

3. How does the factor structure underlying the ILS change during two stages of the MBBS course?

4. How consistent or variable are students' learning strategies, conceptions of learning and learning orientations?

5. How do first and fourth year students perceive their learning environments? 


\title{
5. Beliefs and views of Sri Lankan new entrant medical stu- dents on learning
}

\author{
Introduction
}

\section{Individual differences in learning}

Within the field of academic learning, over the last three decades or so, individual differences in learning have been categorized under different terms, such as learning approach (Entwistle, 1997), orientation to learning (Entwistle, 1997; Lindblom-Ylänne, 1999), learning style (Vermunt, 1998), and study orchestrations (Meyer, 2000). Recently, Vermunt and Vermetten (2004) preferred to use the term learning pattern in place of learning style (see Chapter 3). Vermunt $(1996,1998)$ in his work identified interrelationships between the four main components of learning, namely:

1. the cognitive processing activities, which are the thinking activities that students employ to learn;

2. the regulating activities, which deal with orientating and organizing oneself as a learner to achieve the set goals, developing the ability to cope with the feelings that arise during the learning process;

3. the conceptions of learning and knowledge, which govern the task division between the teacher and the learner in learning and

4. the learning orientations, which are the personal goals, intentions, motives, expectations, attitudes, worries and doubts of students.

Vermunt $(1996,1998)$ used the term "learning style" as a coordinating concept in which the interrelationships between cognitive, affective and regulative learning activities, learning conceptions and learning orientations are united. Several researchers (Busato, Prins, Elshout, \& Hamaker, 1998; Vermetten, Vermunt, \& Lodewijks, 1999a; Vermunt, 1998) who have used the ILS have identified four learning styles with the ILS, i.e. reproduction directed, meaning directed, application directed and undirected. However, Vermunt and Vermetten (2004) reported that in some instances coherent linkages between the four learning components were absent. Beishuizen, Stoutjesdijk, and Van Putten (1994) had documented that students who combined deep processing with self-regulation and those who combined stepwise processing and external regulation performed better than those who used incompatible learning strategies components. In a literature review on the subject of internal coherence among the learning components assessed by the ILS, Vermunt and Vermetten (2004) pointed out that the associations among learning strategies students use and their learning conceptions and orientations become strengthened with progression of education as well as with adaptation to a particular learning context.

This raises questions about the relation between these variables at the other end of the continuum of medical education, i.e. at the entrant level. However, the individual difference in learning among Sri Lankan new entrant medical students has not been studied in the recent past. Entrant medical students are in a transition phase having left the senior secondary education where the schools and often the tuition classes have prepared them for the extremely competitive national examination, which they have passed with high scores. They are about to enter a new phase of education, which is likely to demand new ways of studying. The university teachers as evidenced by the discussions within the fac- 
ulty, about curriculum reform, hoid low expectations about the independence and self directed learning skills of these new entrants. Gaps between actual skills and mutual expectations may work out in different ways; positive when both groups are prepared to move on to a next stage of self directed learning and negative when expectations are too high or too low (Vermunt \& Verschaffel, 2000). The research presented in this chapter seeks to clarify the learning strategies, learning conceptions, motives and expectations of new entrants. In the research presented here a qualitative approach is taken. Such qualitative approach was needed because no validated instruments exist for the given population. And most importantly, we wanted to look at the learning process and environment from the perspective of the learner.

\section{Qualitative research methods}

As explained in Chapter 3, the trend in higher education is to explore the learning context through the eyes of the learner. Thus, qualitative studies have been used extensively to gather information on various aspects of student learning (Dornan, Hadfield, Brown, Boshuizen, \& Scherpbier, 2005; Kember \& Gow, 1990; Lindblom-Ylänne \& Lonka, 1999; Marton, Dall' Alba, \& Beaty, 1993; Vermunt, 1996). Exploring what learning means to the learner, how it is experienced, understood or conceptualized by him or her is a relatively recent, but expanding area of research (Marton, Watkins, \& Tang, 1997).

In educational research, interviews are increasingly used to both validate questionnaires developed in different cultures and to gather relevant information on teaching and learning (Ajisuksmo, 1996; Gow \& Kember, 1993; Oosterheert \& Vermunt, 2001; Trigwell \& Prosser, 1996; Vermunt, 1996). According to Thomas (1998), talking with informants enables researchers to gather diverse types of information, for example, informants' patterns of thought, abililies, judgments of other people and recall of events. Advantages of interviews over printed questionnaires are many. Unlike questionnaires, interviews permit the researcher to rephrase questions that respondents do not understand and also allow respondents to elaborate their ideas at length. This method further enables the researcher to pose questions in a general form, particularly offering respondents freedom to tell what a particular word or phrase means to them (Thomas, 1998).

Phenomenography as highlighted in Chapter 3 is a research methodology that has been employed by many educational researchers to analyze the data obtained by extensive interviews or written open questions (Marton \& Säljö, 1997; Marton et al., 1997; Oosterheert \& Vermunt, 2001; Trigwell, Prosser, \& Waterhouse, 1999; Vermunt, 1996; Zanting, Verloop, \& Vermunt, 2001). The aim of this approach is to reveal the different ways in which people experience or conceptualize various phenomena in the world around them. During the analysis of the interview transcripts the researcher basically looks for consistencies and differences across the responses obtained for a specific question and later within an individual's responses. Phenomenography thus, attempts to provide a description, which not only characterizes different conceptions of learning but also searches for relationships both within and between conceptions (Marton et al., 1997).

\section{Role of interviews and scope in the present study}

The interview approach was deemed to be the most appropriate method to gain an insight into the way students experience their study context and how they think about the key features of learning and studying. As stated above interviews enable the researcher to collect diverse information, such as informants' patterns of thought, abilities, judgments of other people and recall of events. Accordingly, the interviews are capable of exploring the 
study habits of students (including whether it is the learner or the teacher/ instruction that sets the limits of study and monitor the process) at entry, their ways of attributing academic successes and failures, their ideal image of a student, their motives behind the selection to study medicine, their expectations on the teaching and learning process in the university and beliefs and views on good learning. Therefore, the data collected by means of interviews can be used to identify different categories of description for the four main components of learning described by Vermunt (1998): cognitive processing activities, regulation activities, study motives and conceptions of learning as well as factors which facilitate learning and attribution pattern among Sri Lankan students.

\section{Research questions}

The interview study described in this chapter regarded three main questions: what is good and ideal learning in the eyes of the students, what are perceived aspects of present and future learning, and do these hang together in a certain way? These questions have been worked out to the following sub-questions:

1. What are the attributes of "an Ideal Student" from the perspective of Sri Lankan entrant medical students?

2. What are factors that have facilitated learning so far among these novice medical students?

3. Which cognitive processing activities did these students employ during their senior secondary education?

4. To what degree do students report internal and external control of the learning processes at the commencement of the undergraduate studies?

5. What kind of conceptions of learning and teaching do they report at the commencement of university education?

6. How do entrant Sri Lankan students attribute academic success and failure?

7. What are their expectation of learning and teaching in the university?

8. What motives underlie their selection of the medicine course?

9. Do the students' descriptions of the components of actual learning (cognitive processing, regulation, conceptions of learning) and expected learning show any associations?

\section{Method}

\section{Participants}

A random sample of 35 new entrant medical students, following the Intensive English Course, during the period July to September 1999, at the Faculty of Medicine, University of Peradeniya (FMUP), Sri Lanka were selected from the admission list. After repeated reminders 32 (response rate of $91 \%$ ) students turned up for the scheduled interviews that were held after faculty hours. The interview sample consisted of 17 male $(53 \%)$ and 15 female (47\%) students. The mean age of the sample was 23 years. A small majority of the students $(56 \%)$ reported that they secured admission to the medical faculty in their first attempt at the entrance examination. The aggregate scores obtained by the student sample in order to be selected to the stream of medicine varied between 268-315 out of a total of 400 marks. The sample consisted of students who have secured admission to FMUP from educationally advantaged districts (75\%) as well as $25 \%$ from disadvantaged districts (see Chapter 2). The majority of the students were from middle class backgrounds (based on the parental occupation and stated monthly family income). 


\section{Interview schedule}

A broad examination of learners' views on teaching and learning issues was thought to be valuable. Hence, a semi structured interview schedule based on the topics of the ILS scales was prepared. Accordingly, the first question of the interview introduced the interviewee towards an area of interest; the following questions were broad, open-ended questions directed at finding out what seems uppermost in the respondents' mind in relation to the topic at hand. For each open question, a set of additional probes was formulated to make sure that all relevant aspects were covered by the respondents. The interview was conducted in such a way that the respondent's pattern of reasoning and motives could be explored. Critical incidents were described and analysed to elicit the students' conceptions of good learning. The interview schedule covered areas such as good learning, study strategies during senior secondary years, conception of learning and knowledge, attributions of academic successes and failures, expectations of teaching and learning in the University, the "ideal student" and study motives.

\section{Procedure}

Ethical approval for the study was obtained from the Ethical Review and Research Committee of the FMUP. The next step was to prepare the interview schedule and test its feasibility. Two pilot interviews were held before the final version was developed. The interview schedule was followed as described above and the interviews were conducted after teaching hours by the researcher in her room, prior to the commencement of the academic course. The interviewee was given the choice of selecting the language (Sinhala or English) that he or she was comfortable with, to express his or her ideas fully. According to Bennet (1977) the communication language affects the quality of the interaction. He concluded that when bilinguals are allowed to interact in their native language, they give more favourable responses and thus, he was of the view that language evokes a reference group. Each interview lasted for about 45-55 minutes. Informed consent was obtained prior to the interview. Student participation was voluntary and respondents were not rewarded for their participation in any way.

The interview was mainly directed at exploring the views of students' on learning, their study strategies, motivation and their expectations of the learning process in the University. However, the students were free to raise any issue of interest to them, while any prompting by the interviewer was designed to clarify or elicit further information. An effort was made to avoid leading the participant. All the interviews were tape-recorded.

\section{Data analysis}

All interviews were transcribed, translated into English, entered into a word processor and printed. This resulted in 225 pages of transcript and they were a rich source of data. In the analysis of most of the data phenomenography (see earlier in this chapter) was the research method adopted. The exception was the quotes covering students' attributions of successes and failures and conceptions of learning and knowledge, the analysis of which was based on predetermined categories as described later.

First, all interviews were read completely. Subsequently the interviews were studied several more times, during which phase quotes were selected that were relevant to the research questions. The quotes were next organized in a word processor under the broad areas relevant to the questions i.e. facilitating learning, learning activities etc., and for each quote an identification number was given. These quotes were used in the subsequent 
Chapter 5

analyses to derive the phenomena and the categories and subcategories in relation to the different learning components, based on the consistencies and differences among them. The researcher also examined how different learning components are related to each other within an individual learner by means of a cross table.

A different approach was taken in the analysis of the conceptions of students on learning and knowledge. Here the scale described by Lonka, Joram and Bryson (1996) was used as a guideline for the analyses (see Table 5.1).

Table 5.1. Scalcs used to analyze participants responses on learning and knowledgc.

Constructivity

1. Storing existing knowledge: Learning seen as absorption, intake of knowledge, or simply storing (4.1.1).

2. Assimilation: Learning vicwed as assimilating knowledge into a pre-existing framework or interpreting knowledge within an existing framework (4.1.2).

3. Change or reorganization takes place: Learning seen as changing thinking or reorganizing knowledge; learning is an interplay between assimilation and accommodation (4.1.3)

4. Construction of new knowledgc: Learning seen as changing or reorganizing plus constructing new knowledge, knowledge structures, or new ideas $(4.1 .4)$.

Active epistemology

1. Learner viewed as object of education: Learning is absorption of information; to learn is to be taught (4.2.1).

2. L.carner seen as implicitly passive: Leaming is acquisition of information; a change occurs (4.2.2).

3. Learner seen as implicitly active: An active verb is used to describe learning, e.g.. "learning is using mental abilities": learning is viewed as problem solving or as discovery (4.2.3).

4. Learner emphasized as being active: Learning is explicitly described as an active process $(4.2 .4)$.

Mental representations

1. No notion of represcntation: No representational terminology used (4.3.1).

2. Folk notion of representation: Beliefs, thoughts, meanings change when learning occurs (4.3.2).

3. Notion of representation implicitly applied: Using a phrase such as "change in the storehouse" idea of representation evident, but cognitive terminology not used (4.3.3).

4. Notion of representation explicit: Representational terminology used: "knowledge structures," "schemala," etc. (4.3.4)

(Source: Lonka, Joram, and Bryson, 1996, p. 246.)

Lonka et al. (1996) found that students' ideas about learning and knowledge vary over three axes: the level to which learning is seen as active construction (the Constructivity axis); the level to which the student is seen as an active agent in education (the Active epistemology axis); and level of sophistication of the opinions about cognitive effect of learning (the Mental representation axis).

Interview parts regarding how the students attribute academic success and failure they had experienced, were coded on the basis of Weiner's model of attribution (see Table 5.2). 
Table 5.2. Attribution patterns for success and failure in Weiner"s model of attribution with examples of statcments.

\begin{tabular}{|c|c|c|}
\hline \multirow{2}{*}{$\begin{array}{l}\text { Dimensions of classification of } \\
\text { attributions }\end{array}$} & \multicolumn{2}{|c|}{ Examples of reasons given for } \\
\hline & Success & Failure \\
\hline Internal stable uncontrollable & High aptitude & Low aptitude \\
\hline Internal stable controllable & Always study hard & Never studies \\
\hline Internal unstable uncontrollable & Good health & $\begin{array}{l}\text { Sick the day of the } \\
\text { exam }\end{array}$ \\
\hline Internal unstable controllable & $\begin{array}{l}\text { Worked hard this } \\
\text { time }\end{array}$ & $\begin{array}{l}\text { Did not study for this } \\
\text { particular test }\end{array}$ \\
\hline External stable uncontrollable & $\begin{array}{l}\text { Conducive school en- } \\
\text { vironment }\end{array}$ & $\begin{array}{l}\text { School has hard re- } \\
\text { quirements }\end{array}$ \\
\hline External unstable uncontrollable & Lucky & Bad luck \\
\hline External unstable controllable & Friends helped & $\begin{array}{l}\text { Friends } \\
\text { failed to help }\end{array}$ \\
\hline
\end{tabular}

So a student's explanation "I could not perform as expected in the 1st term because I had not studied all the subject areas covered in that term ... I have not made sufficient effort to write answers to model questions prior to the examination", was coded as an internal, controllable and unstable attribution.

To investigate the relation between learning conception, learning strategy, regulation strategy, study motive, and learning expected in the university an overview table (see Appendix 1) was constructed using the categories developed earlier to search for distinctive patterns. Vermunt's findings on integration between learning conceptions, motives and study strategies were taken as a heuristic to identify patterns (Vermunt \&Verloop, 2000 ). According to them theoretically congruent learning patterns are; expressing an intrinsic motivation, constructive conception of knowledge and reporting the use of deep level processing strategies and self regulating the learning process or expressing a certificate orientation along with a knowledge assimilating type of conception, the use of surface learning strategies and relying on external regulation of the learning process. Similarly expressing a conception of use of knowledge, vocation orientation and indicating use of concrete processing is yet another consonant pattern.

\section{Results}

The interview transcripts were analyzed as stated. Categories and subcategories were identified under each heading related to the separate research questions, such as attributes of the ideal student, good teaching and learning in the eyes of students, the cognitive processing strategies and regulation strategies of new entrants during high school education, as well as expectations of university teaching and learning. Conceptions of learning, the attributions of academic success and failure and learning orientations were also identified by the initial analysis. The different categories and subcategories are described with the help of relevant interview quotes. A complete overview is presented in Appendix 1 with the inclusion of examples indicating the degree of variation and depth of interview responses and a table depicting summarized interview data. 
Chapter 5

\section{Attributes of an ideal student}

The student views of an ideal student exposed the understood goals of education, which the target group may strive to achieve. Broadly speaking, the student descriptions of the "ideal student" show the following dimensions;

1.1 BROAD KNOWLEDGE BASE - Some students (9) expressed that the ideal student has a broad knowledge base, not just what is taught in class. (See appendix 1.a for relevant student quotes.)

1.2 USE OF EFFECTIVE STUDY STRATEGIES - A majority of the students (22) expressed that an ideal student engages in constructive learning strategies such as concretizing, critical processing and self-regulation of learning (1.b.1a) Such students also self regulate the learning process and manage resources efficiently (1.b.lb)

1.3 INTRINSIC MOTIVATION - Several students (11) expressed that "ideal student" shows a high degree of personal interest therefore works hard and independently search for knowledge in order to enrich oneself (1.c).

1.4 ATTITUDE- A substantial number of interviewees (24) indicated that the ideal student has some attitudinal or even personality qualities:

1.4.1 A social, outgoing, responsible, cooperative attitude is seen as important. This is contrasted with 'bookworm' or 'does only studies', which is seen as undesirable (1.d.1a).

1.4.2 Commitment to their studies. Does not postpone studies, takes responsibility for learning (1.d.1b).

1.4.3 Have the caring and helping attitudes linked to the medical profession (1.d.1c).

The overall image that permeates through the interviews is that the ideal student combines several of these qualities making him or her an energetic, multi-facetted person as is illustrated by the following interview quotes.

(s 2): "An ambitious person, who also has a broad knowledge. Somebody who knows correct facts... A responsible person too." For this student broad knowledge base, motivation and attitude are the important features.

(s 15): "A person who thinks in depth, apply the knowledge practically, carry out work to achieve a set goal, not a "book-worm" but also participate in extra curricular activities and maintains good interpersonal relationships." This description combines effective study strategies, and desirable attitudes.

(s 21): "A methodical student. Somebody who covers up the subject areas dealt on that day. A balanced person devotes time for both academic and extracurricular activities, relaxed and mentally stable. ... Good conduct, obedient to parents." Again a combination of effective study strategies and attitude. 
(s 24): “... an ideal student is somebody who does not postpone his studies, very methodical. Very inquisitive. Interested in studies, search for knowledge, reads a lot of books. Thinks a lot. Does not believe things just because somebody tells that to him but uses his knowledge and accepts what is being told". This student used three characteristics: effective study strategies, intrinsic motivation and attitude.

\section{Factors facilitating learning}

The student descriptions of a good learning experience included two main facilitators of learning: good learning (code 2.a; 26 statements) and good teaching (code 2.b; 25 statements). The new entrant students considered the following activities as examples of Good Learning:

\subsection{Good learning}

2.1.1 ACTIVE ENGAGEMENT IN LEARNING - Ten (10) students pointed out that whenever the teacher (often the teacher in tuition class) provided opportunities for them to be intellectually active that resulted in meaningful learning. Some of the students expressed that effective learning occurred when the learner was made to think or do various things than made to passively listen to other people's versions (2.a.1a).

2.1.2 COOPERATIVE LEARNING - A few students (2) attached great value to cooperative learning. Discussions with one's peers enabled one to clarify difficult subject areas and thereby promoted understanding. It was also interesting. Each time one presented his/her point of view, one's own knowledge was being clarified and broadened. They believed that arguing with friends helped to consolidate one's knowledge (2.a.1b).

2.1.3 SELF DIRECTED LEARNING - Five (5) students indicated that they preferred situations where the learner had to search for knowledge and learned without that much of teacher guidance. In such a case learning became more meaningful, as was stated by this student. (2.a.1c).

(s 15): "Whenever we learn things by searching for information or experimenting on our own that kind of thing can be easily retrieved"

2.1.4 PRACTICALS - Some students (8) indicated that effective learning occurred when the individual was given the opportunity to have hands-on experience. It was thought to be beneficial when the teacher provided an appropriate learning environment that allowed the students to have direct interaction with learning resources and where the students could be involved in learning with more senses than in the normal classroom (2.a.1d).

\subsection{Good teaching}

Also the effect on learning imposed by the learning context (teacher and instruction) was highlighted by a considerable number of students (16) during the interview. Some teachers who were capable of facilitating learning in students were much sought after 
by students. This could be either due to the way the particular teacher had planned the lesson, skillfulness of the teacher in executing teaching functions, arousing interest and ability to explain complex things in a simple manner. As for good teaching the categories emanating from the interviews are the following;

2.2.1 GIVING APPROPRIATE FEEDBACK - A few (3) students indicated that teacher feedback on their mistakes and on correct points of view helps them to develop as a learner. They use it to analyze and correct their knowledge and performance (2.b.1a).

2.2.2 MAKING COMPLEX THINGS EASIER TO UNDERSTAND - Some students (6) also saw it as good teaching when the teacher simplified the subject matter for them by connecting complex things to more simple things students know already, by schematizing the learning materials, giving illustrations or referring to examples (2.b.1b).

2.2.3 HELP CONSTRUCT KNOWLEDGE - Some teachers helped the student (8) to build up knowledge in a logical manner by clarifying the difficult areas, not by simplifying the learning materials, but by asking questions, discussion, or using examples and counter examples (2.b.1c).

2.2.4 MOTIVATION - Good teaching had several effects that led to good learning, directly through understanding and meaning construction, and indirectly through improved motivation and confidence. Four (4) students linked good teaching to motivation (2.b.1d.1). Two other students reported that the teacher by allaying anxiety and improving confidence alone motivated them to learn that particular subject and sometimes other subjects as well (2.b.1d.2 \& 2.b.1d.3).

\section{Study strategies}

As far as study strategies are concerned students describe their learning in terms of cognitive and of regulation strategies. Regarding the cognitive strategies the following categories were identified:

\subsection{Cognitive processing strategies}

3.1.1 SIMPLY MEMORIZING FACTS - Some students (4) indicated that they simply memorized facts. Often this strategy was used when the situation asked for it in their view: It was a requirement to obtain higher marks; or simple facts asked for simple processing. This strategy was never seen in isolation. Many people used it next to other strategies (3.a.1a).

3.1.2 COMMIT TO MEMORY AND UNDERSTAND - Most of the students (27) revealed that they were provided with a set of notes, which the respective teachers have prepared. Students are advised to read and re-read those as the note covers the important areas that could be asked at the examination. So the students read the notes over and over again and slowly developed an understanding. They stop this process when they do understand the material and can fully remember it (3.a.1b). 
One student indicated that this strategy does not work out for him.

(s 27): "I can remember one teacher asking me to read the note as many times as possible so that $I$ will remember better, but I could not try that, I preferred my method of listening to the teacher and reading a book, then making my own notes and flowcharts. I could understand better this way".

3.1.3 STUDYING THE SUBJECT MATTER STEP BY STEP - Two students (2) expressed the view that he/she first studied one section, finishes that, and then moves on to another section. Since the teaching and assessment are held in a subject wise manner this method is helpful for some of the students (3.a.1c).

3.1.4 ACTIVELY RELATING AND STRUCTURING OF INFORMATION Several students (10) mention that while they study they make it a point to compare different parts of subject matter and organize parts of knowledge into wholes (3.a.ld).

3.1.5 APPLYING WHAT IS LEARNED - Seven (7) students said that they applied the theory in practice and that helped them to understand and concretize the knowledge (3.a.1e).

\subsection{Regulation strategies}

3.2.1 EXTERNAL REGULATION OF LEARNING PROCESSES AND RESULTS - Half of the students (17) indicated that their learning process was to a great extent externally regulated because, as they say, that is a requirement to obtain very high marks at the selection test.

3.2.1.a The tuition class teacher

All the interviewees had attended tuition classes. They indicated that they learned considerably in these classes. The tuition teachers make them particularly aware of what is important from the point of view of the public examination. The teaching style of some teachers has motivated the students. Furthermore, the tuition teachers coach the students to obtain highest marks at the examination by teaching them how to write the answers. Thus, tuition classes can totally regulate the students' learning, but this effect can be mitigated because students themselves can choose their own tuition teacher and class based on their self-perceived needs (3.b.1a.1). On the other hand, several students (11) said that they studied everything the teachers taught without questioning. They let the learning process be controlled totally by instruction (3.b.1 a.2).

\subsection{1.b Past paper questions}

About five students reported that some examination questions were repeated; therefore they could learn and thus score higher marks by concentrating on the past paper questions. Taken to the extreme this strategy can work even without really understanding the subjects (3.b.1 a.2). 


\subsection{1.c Monitor advancement through past papers questions}

Many (14) students reported that they used their scores on past papers and model answers to monitor their progress and to inventory weaknesses (3.b.1a.3).

\subsubsection{SELF - REGULATION OF LEARNING CONTENT AND PROCESS -}

The interviews also indicated that many (22) students to some extent selfregulate their learning. Planning, executing and monitoring one's learning process was evident in their descriptions. Remarkably, external and selfregulation of learning content and process were not mutually exclusive. Several (12) students who indicated that their learning was externally controlled also showed process self-regulation, e.g. of content that was preselected by the teacher.

Many learners (22) indicated that they made their own decisions on what areas to study, what books to read and what methods to adopt during studying and that they planned their time effectively (self regulation of learning process pre-selected by self). Students, themselves choose cognitive processing activities such as selecting, relating and structuring and concrete processing. In those cases they have also derived ways of monitoring their study progress.

3.2.2.a Monitoring study progress

Contrary to monitoring progress by means of past paper questions, a substantial number of students (18) regulated their learning by internal means. They guide themselves with questions that they ask during studying (appendix 3.b.1b.1 \& 3.b.1b.2).

3.2.2.b Planning the study process, effort and organization

Several (11) students indicated that they regulated their learning by being methodical, working according to a plan, not postponing things and taking the trouble to get feedback and modify the plan accordingly (3.b.1b.2.1; 3.b.1b.2.2).

3.2.2.c Self-selecting the learning activities to deepen understanding Among the activities mentioned were:

3.2.2.c.a Reading textbooks or articles (16 statements: 3.b.1b.3.1).

3.2.2.c.b Making their own short notes or charts and diagrams, connecting the relevant areas (14 statements: 3.b.1b.3.2).

3.2.2.c.c Asking for clarification of problematic areas with teachers or students (11statements: 3.b.1b.3.3).

\subsection{Cooperative learning}

The interactions with friends were thought to be a good learning strategy by eight students. Argument was considered particularly useful. It seems to combine internal and external regulation as shown by the following quote. 
(s 20): "After answering questions from a particular area the friends got together and discussed and argued over the answers" (see appendix 3.c.1).

\section{Conceptions of learning and knowledge}

In respect of the amount of constructivism in the participant definitions of learning and knowledge (as shown in Table 5.3), the most commonly expressed views were that learning is a process of reorganizing knowledge (4.1.3; twelve interviewees) or construction of new knowledge and learning was seen as changing, reorganizing plus construction of knowledge structures or ideas (4.1.4; 9 of the interviewees).

Table 5.3. Percentages of students who expressed certain conceptions of learning and knowledge measured with Lonka et al. (1996)'s scales.

\begin{tabular}{|c|c|c|c|c|}
\hline 4.1 Constructivity & 4.1.1 Storing & 4.1.2 Assimilation & $\begin{array}{l}4.1 .3 \text { Change/ reor- } \\
\text { ganization }\end{array}$ & 4.1.4 Construction \\
\hline $\begin{array}{l}\text { 4.2 Active episte- } \\
\text { mology }\end{array}$ & $\begin{array}{l}0 \% \\
\begin{array}{l}\text { 4.2.1 Object of edu- } \\
\text { cation }\end{array}\end{array}$ & $\begin{array}{c}22 \% \\
\begin{array}{c}\text { 4.2.2 Implicitly } \\
\text { passive }\end{array}\end{array}$ & $\begin{array}{l}37.5 \% \\
4.2 .3 \text { Implicitly ac- } \\
\text { tive }\end{array}$ & $\begin{array}{c}28 \% \\
\text { 4.2.4 Pronounced } \\
\text { active }\end{array}$ \\
\hline $\begin{array}{l}4.3 \text { Mental repre- } \\
\text { sentation }\end{array}$ & $\begin{array}{l}0 \% \\
\text { 4.3.1 No representa- } \\
\text { tion }\end{array}$ & $\begin{array}{l}18.7 \% \\
4.3 .2 \text { Folk termi- } \\
\text { nology }\end{array}$ & $\begin{array}{l}59.3 \% \\
4.3 .3 \text { Implicit cogni- } \\
\text { tive }\end{array}$ & $\begin{array}{c}22 \% \\
\text { 4.3.4 Explicit cog- } \\
\text { nitive }\end{array}$ \\
\hline & $3.1 \%$ & $96.8 \%$ & $0 \%$ & $0 \%$ \\
\hline
\end{tabular}

Note: Percentages have been taken from the total number of intervicwecs. One student can have expressed no, one or more than one conception per scale. Hence totals per row do not have to count up to $100 \%$ : totals per column are meaningless.

Seven out of thirty two interviewees also felt that learning is assimilation of knowledge. However, no one voiced conceptions that learning is the same as storing knowledge (4.1.1), while some students explicitly rejected this idea. The following quotes nicely illustrate this opinion:

(s 14): " ".... developing a correct understanding of things that are necessary for the future. It is a continuous process. Learning is something that is constructed by an individual. It is not memorizing what is written in a book in its original form. It is a form of making knowledge more meaningful to oneself."

(s 29): "Not to memorise word to word but to make things meaningful to me. We learn so much in day to day life with experience."

(s 1): "... learning is not just studying something and repeating that at the exam, one must develop an understanding."

On the active epistemology scale the learner was viewed as implicitly passive by six interviewees (4.2.2) and implicitly active (4.2.3) by nineteen of the medical students while another seven students (4.2.4) emphasized that the learner is explicitly active in the learning process. For example; 
(s 16): "Learning is something that is constructed by an individual. It is not memorizing what is written in a book in its original form. It is a form of making knowledge more meaningful to oneself."

(s 11): "Knowledge consists of facts gathered from the environment. These are obtained by different means. Knowledge changes as a result of further thinking."

(s 23): "Learning by actual experience from the environment under the guidance of somebody. Think about the experience and try to understand the fundamental things in relation to actual practice. If the learner has learnt something that person will use that in practice and learn more from those experiences."

Finally, the majority of students (31) used a folk notion of representation (4.3.2). They expressed the view that beliefs, thoughts and meanings change when learning occurs. However, none of the medical students explained learning using standard terminology such as schemata or knowledge structures. Instead, the expressions were;

(s 16): "It is a continuous process, a change in the way of thinking occur."

(s 21): "Learning also results in change of attitudes and behaviour of students, the knowledge obtained depends on the status and capabilities of the individual"

(s 32): "Changing the way of thinking if that is not correct."

(s 19): "Beliefs, thoughts change as a result of learning."

\section{Attributions of academic success and failure}

\subsection{Success}

Many students expressed their pride and happiness about academic success, often their admittance to medical school. Students in general (30) attributed success to hard work, an internal, controllable and unstable cause as stated in the examples below and under category 5.a. in the appendix.

(s 14): "I was very happy. I think this is the result of studying hard".

(s 17): "I worked hard much more than the first attempt. This time I concentrated on past paper questions that helped me because some of the questions were repeated."

Implicit in this statement is the fact that this student has not faired well in the previous attempt as has not worked hard and has been successful this time due to hard work and some luck.

\subsection{Failure}

A large majority of the students (25) was found to make internal, controllable and unstable attributions of an academic failure. The results were consistent with the expectations, as most of the students have tried again and finally succeeded. 
(s 6): "This happened during the end of term examination. I thought I have not studied enough. I met the school teachers to find out my deficiencies and decided to study harder".

This quote also nicely demonstrates that controllable, unstable and internally located attributions in case of failure act as a motor to try again and do better (see appendix 5.b. for similar statements).

One student reported that he has not faced a failure so far and he could not describe such a situation. Two students thought the failure was due to an external cause such as teachers not teaching properly or having to travel a long distance from home. One student attributed the failure to bad luck, an uncontrollable cause.

\section{Student expectations about learning in higher education}

6.1. GREATER EFFORT - Many (24) students expected that they have to work hard in medical school, but some are not outspoken on this subject. Relevant interview excerpts are given below and in appendix 6.a.

(s 26): "I think we will have to work hard. Teachers will help us, but we will have to do a lot of self-study. I know this is difficult at the beginning but I feel that this method is more effective".

6.2 DIFFERENCES WITH SCHOOL - Most of the interviewees (31) assumed that studying in the university would be different from learning in school, although one student assumed that university would not be so different from school.

Expected differences concern the learning materials, regulation, level of understanding and practical application of knowledge and skills acquired, and discussion and interaction. Accordingly, the role of the teacher will be different as well (see section $7.1 \& 7.2$ ).

6.2.1 The learning materials

Many students (21) expected a change in the kind of learning materials, from teacher notes to books, articles and own notes. There are indications that despite high expectations, some (6) of them express their worries that this might be difficult, or at least 'interesting'.

(s 22): "I do not think that university teachers will provide lecture notes to us. We will have to do a lot of independent study. We will have to refer books. I do not mind referring books but when we have to write our own notes it is not going to be easy. We will have to spend a lot of time and effort to make our own notes. So it is not like the school days where the tuition teachers provide a set of notes; now onwards we will have to take the initiative." (See appendix 6.b.1a.1-6.b.1 a.3 for more excerpts).

6.2.2 Student is responsible

Several students (14 students) also expect that there will be a shift in responsibility for the regulation of the learning process from teacher to student. They expect 
that it will be their own responsibility to plan, prepare and get the work done. Some of students (8) admitted that in senior secondary education either the schoolteachers or the tuition teachers shouldered the major part of the responsibility of learning. They provided notes, questions, answers to questions etc. However in the university, the teachers will not come behind you and provide all the material, so the student will have to take the initiative and actively construct knowledge (6.b.lb).

(s 3): "It is going to be very different to school. Schoolteacher gives notes, diagrams, and everything to us. In the university the student has to be entirely responsible for his/her learning. I think it is going to be interesting".

(s 4): "Unlike in school the responsibility of learning is shifted towards student. During Advanced level days student plays a small part it is the teacher who drives the student".

6.3 LEVEL OF UNDERSTANDING AND PRACTICAL APPLICATION OF KNOWLEDGE AND SKILLS ACQUIRED

Some students (6) emphasized that for them university means that they have to acquire a deeper level of understanding and more practical application of what is learned. In their opinion less emphasis will be placed on rote learning (6.c).

(s 6): "We must attempt to understand the logic behind what is taught, practical applications of knowledge gained and get rid of memorizing facts for its own sake".

This student also thinks that active learning is good, so university education will be a welcome development.

6.4. FROM RECEPTION TO DISCUSSION - Some students (7) assumed that the education program would include many discussion sessions. Discussion classes would be a major teaching method in the medical school, while some think that the students will have to organize that by themselves (6.d).

(s 2): "We will have a lot of opportunities to discuss with students and teachers".

\section{Student expectations of the role of a university teacher}

The students' expectations are not unequivocal; they describe roles ranging from the classical task of a teacher to that of stimulating education by establishing a good rapport with the students, giving constructive feedback, and training the students to plan and organize their work leading to more independence.

7.1 ENABLE SELF-REGULATION - Teachers play a major role in ensuring learning in their students. A majority (17 statements) indicated that teachers should not take over the learning functions of students but take measures to empower the students (7.a). The excerpt below is a comparison of the situation in school and what is expected. 
(s 1): "Unlike the teachers in schools who force us to study and then dictate notes and shoulder greater part of the responsibility of learning, we must be instructed to do certain things, refer books, may be discussions encourage us asking questions for clarification purposes and make us think and work independently..."

7.2 SUPPORT LEARNING - Most students (26) uttered the idea that university teachers should support their learning by establishing a relationship with their students, and by being approachable and accommodative. A teacher should take the trouble to discuss the strengths and weaknesses of students; "function as a diagnostician" was implicit in some answers (see 7.b for more examples).

(s 1): "Unlike the teachers in schools it is expected that there will be a good relationship, ensure equality they will encourage discussions and interactive learning and be approachable, friendly and be flexible, respect views of others."

(s 6): "Point out the weaknesses of students and provide constructive feedback".

7.3 FUNCTION AS A "TEACHER" - A more school-based view of a teacher was also expressed (5). Some students also expect teachers to cover the syllabus, to explain subject matter in a simplified manner and to provide handouts to them. They prefer a more teacher-centered approach, as was explicit in the statement given below and in 7.c in the appendix.

(s 7): "Teacher must cover the syllabus".

\section{Study motives}

The students had very diverse reasons for selecting to study medicine. Motives can be differentiated into intrinsic and extrinsic. Intrinsic when the learner himself or herself make the decision, extrinsic when the decision is influenced by external factors. Each category could be further divided into those with a major emotional component and those that are related to job or study.

\subsection{Extrinsic motives}

8.1.1 EXTRINSIC MOTIVE - WITH A STRONG EMOTIONAL COMPONENT - Half the students (17) indicated that the wish to satisfy one's parents, sometimes to satisfy teachers and the other family members, had played a role. This motive was often combined with other, intrinsic motives. In this case parental support had a positive influence (student 9 below and 8.a.1a).

(s 9): "From my childhood I had this liking..., wanted to be a doctor. There was this feeling in my family that at least one member should be a doctor. My brother is an engineer and sister in the agriculture faculty. So my parents also encouraged me all the time to pursue a medical career. I also knew that I am good at studying de- 
scriptive subjects and since I could work hard, I would not have a problem with the medical course... Social status of a doctor also influenced my decision"

However, in case other motives were lacking, a choice made to please one's parents seemed counterproductive.

8.1.2 EXTRINSIC JOB RELATED MOTIVES - Several students (11) also mention motives that have to do with desirable aspects of the medical profession.

\subsubsection{Social status of a doctor}

Doctors have a relatively high status and seem to live a comfortable life. Such a future was attractive to several students (9). (See 8.a.lb for more examples.)

(s 15): "I developed a liking for this field about two to three years before I sat for my $\mathrm{O} / \mathrm{L}$ examination...I think I was influenced by the social status of a doctor. Nobody forced me but I decided to select medicine. I also think that doctors can practice till death and the only other profession that could be practiced for a very long time is law. I think this liking for medicine has developed over a period of time."

\subsubsection{Independence at work}

Two students valued the relative independence at work and thus preferred a medical career.

(s 3): "There is a lot of independence at work unlike in banks, private ....etc., One could either practice or do research as it is a vast field."

(s 20): "... I still want to do medicine because I think there is lot of independence. I don't like to work under people. I think I will have more freedom as a doctor."

\subsection{Intrinsic motives}

Other reasons for selecting medicine can be motives rooted in their past, with a strong emotional component and motives related to the medical profession, and motives related to academic satisfaction of the study.

\subsubsection{INTRINSIC WITH A STRONG EMOTIONAL COMPONENT}

\subsubsection{Childhood dream}

Fourteen respondents described motives dating back to their childhood, showing that the motivation can be very persistent: (see 8.b.la for more examples.) 
(s 17): "I like this job. It is a childhood dream. I was determined to do medicine."

(s 9): "From my childhood I had this liking. I wanted to be a doctor."

(s 5): "From childhood I wanted to be a medical officer. Public have a special respect to medical officers."

\subsubsection{INTRINSIC MOTIVES RELATED TO JOB OR STUDY}

\subsubsection{Altruistic motives}

Many (11) students report altruistic motives for their studies. They see serving the sick as very rewarding as indicated in the quote below.

(s 14): "Rather than being an engineer I felt that I would derive a lot of satisfaction by being a doctor. I think it is more than a profession. I think it is a service. A very rewarding occupation... Results of my actions could be seen then and there. By being a doctor I could render a humanitarian service."

8.2.2.2 Interest in the study or profession

Several students (18) stated that they chose the study out of interest as indicated below.

(s 4): "I am interested in forensic medicine and detective work."

(s 23): "Actually after the O/L results I first followed arts subjects in school, then I did not like that so I shifted to a commerce class. I could not develop a liking for that area too. I did O/L again and then I got better results and I changed my mind to do bioscience. ... So I entered a school at Kurunegala and worked as much as possible and got selected to do medicine. I liked bioscience subjects."

This liking for the profession was often inspired by positive experiences with the profession, sometimes due to illness of the students themselves or a close relative as indicated below and in 8.b. 1 b.

(s 25): "I developed the liking for this profession because of the doctors who treated my sister, who was diagnosed as having aplastic anaemia. Now she is cured. I have a good impression about doctors."

One student revealed a high ego-involvement in his or her motive. Interest was not stated, nor implied.

(s 26): "I want to prove that I am capable".

Most students expressed multiple motives for their choice of medicine, combining intrinsic and extrinsic motives. A student ( $\mathrm{s} 14)$ combined altruistic motive with the intent to satisfy a family wish. 
(s 14): "I had the freedom to select what I want to do for my A/Ls. My family would have liked if I did bioscience. Rather than being an engineer I felt that I would derive a lot of satisfaction by being a doctor. I think it is more than a profession. I think it is a service. A very rewarding occupation... Results of my actions could be seen then and there. By being a doctor I could render a humanitarian service."

The following student (s 19) combined intrinsic and extrinsic motives:

(s 19): "I wanted to become a medical officer from my childhood. During my childhood, I was getting ill frequently and thus I thought I should become a medical officer when I grow up. But later when I was doing my $\mathrm{A} / \mathrm{L}$ examination for the first time, I did not mind doing another job as long as I can serve the society. Nobody forced me to do medicine but the decision was taken on my own. I also thought that my parents would be happy if I become a medical officer. I think I am capable so that I decided to put in more effort and try once more."

The following example also shows close associations with doctors during an illness. The interaction has had an impact on selecting a career, in addition to the altruistic motive. The motive is mainly intrinsic with a strong emotional component and is also based on certain characteristics of the job. The liking for study was inspired by positive experiences with the profession.

(s 12): "From my childhood I wanted to be a doctor I don't know why? I like the medical profession a lot. I myself made the decision to follow the medical course. My parents did not force me to do any thing. I was asked to decide for myself. I like to help people. There was a time when I fell ill I had hepatitis I was a very difficult patient not allowing to take blood and to give injections and so on but the way my doctor tolerated me was very impressive. His approach was such that I was not frightened of those later. When people are sick irrespective of their status they become helpless and anxious. I think it is a service to treat the sick. Being a doctor I can earn a living as well as do a service."

9. Associations between learning conceptions, learning strategies, regulation strategies and expected learning in the university

Analysis of the findings represented in Table 1 of the Appendix section revealed three groups of students. The first group consisted of 13 students reporting learning strategies in balance with their learning conception. Seven (7) of them expressed a constructivist conception of learning and also reported the use of deep learning strategies and selfregulation of learning process as the prominent strategy, thus learning strategies of this group were consistent with their conception of learning (e.g., s10, s11, s13, s14, s25, s28, s30). One student whose learning conception was more of knowledge assimilation (4.2.2) 
reported surface learning strategies and external regulation of learning, which were consistent with the stated conception (s17). The remaining students indicated the use of committing to memory and understanding, which is neither a deep processing strategy, nor exactly a surface strategy along with a constructivist conception and self regulation (e.g.s5, s15, s24, s29, s32). A second group of students (12) failed to demonstrate a coherent relationship between the learning conception and learning strategies. They showed internal contradictions. Some students reported a constructivist conception of learning yet has engaged in not so deep level processing and indicated learning process was under external control $(\mathrm{s} 7, \mathrm{~s} 8)$. One student whose conception was close to assimilation of knowledge reported the use of a deep processing strategy and self-regulation of the learning process (s2). This was the inconsistent group. A third was characterized by the expression of a constructivist conception and the use of both deep and surface cognitive processing strategies and self-regulation and external regulation (e.g., s9, s12, s16, s21, s23,s26, s31). They could be categorised as the full regulation group.

This finding suggests that only a minority of the students expressed conceptions and strategies that were in balance. As a consequence it was not feasible to seek for associations of such patterns with expected learning in the university. Instead an analysis was made to see how learning strategies related to believes of knowledge and regulation and whether opinions about good learning predicted students' expectations of learning in the university. Results suggest the following: Only the study strategies Relating and structuring and Active application of knowledge showed differences between students. About half of the students talked about deeper processing strategies. Only two indicated that they used both. No clear relations between the use of these study strategies with opinions about knowledge (level of constructivity) nor with regulation strategies could be discerned. Exceptions might be that students who relied on both internal and external regulation strategies tended to use more relating and structuring (4 of 5).

Regarding future learning: A large group of students (17) expected that university teachers would support their self-directed learning, though a large majority of the students (27) did not mention self-directed learning as a feature of good learning; a third of them (9) expects that they will be more responsible for their own learning in the university. The five students who saw self-directed learning as a desirable quality were divided in their expectations: two of them expected to be granted more responsibility for their own learning.

The pattern for active learning does not show much relation between opinions expressed about good learning and expectations about active learning in the university. Half of the students (4) who mentioned active learning as a feature of 'good learning' (9) did not mention it when they described their expectations for learning in the university. Conversely, half of the students who did not mention active learning as good learning (10 vs 23 ), expected that their university learning would be more active.

\section{Discussion and conclusions}

The study reported in this chapter was meant to answer multiple questions and to serve multiple goals. One goal was related to the educational reform process in the medical school, which required to have a deeper insight in the experiences, views and opinions of new entrant students; especially the faculty teachers who hold low expectations about the independence and self directed learning skills of these new entrants, need information from different sources than the experiences they gather during lectures and through tests. 
The questions sought to answer were formulated at a rather detailed level:

1. What are the attributes of "an Ideal Student" from the perspective of Sri Lankan entrant medical students?

2. What are factors that have facilitated learning so far among these novice medical students?

3. Which cognitive processing activities did these students employ during their senior secondary education?

4. To what degree do students report internal and external control of the learning processes at the commencement of the undergraduate studies?

5. What kind of conceptions of learning and teaching do they report at the commencement of university education?

6. How do entrant Sri Lankan students attribute academic success and failure?

7. What are their expectation of learning and teaching in the university?

8. What motives underlie their selection of the medicine course?

9. Do the students' descriptions of the components of actual learning (cognitive processing, regulation, conceptions of learning) and expected learning show any associations?

Dealing with these questions in the results section happened at an even more detailed level. For educational reform these detailed answers are very important. However, we cannot say, based on these outcomes whether students' expectations are realistic, neither can we refute or confirm teachers' pessimistic opinion without more explicit expression of their expectations. What we can do is to find out whether there are inbuilt frictions and inconsistencies in the students' patterns of experiences, ideals and expectations to find out whether and where problems can be expected. As a next step we will take a step back and will try to formulate more abstract answers to these questions in terms of commonalities and differences, and in terms of consistencies with theory and findings in other countries, cultures and setting. Next we will address issues related to the methodology applied and consequences for generalisation. Finally we will formulate possible explanations and research questions to be answered in the subsequent chapters.

At a more general level the results suggest the following: Based on their experience in secondary education students have developed concepts of the ideal student and of ideal teaching and learning that seem to hang together. The students emphasise that an ideal student should do more than studying and study more than what the syllabus prescribes; the ideal student should also use effective (not ideal) study strategies, and be intrinsically motivated toward studies and profession, showing so in a caring and helping attitude. Ideal learning would involve active, meaningful and concrete forms. The role of the ideal teacher consists of support and feedback: help understand complex things, help to construct knowledge and (for some students) to give feedback.

The most important finding on processing and regulation strategies was that a large majority of the students described a study strategy that was characterised as 'committing to memory and understand', while about half the students used deep and active strategies. This finding sheds a new light on the students' descriptions of ideal learning and teaching. Students seem to see committing to memory and understand as an effective, though not ideal study strategy. Ideal teaching support or invokes this style; their ideal learning regards the deeper and more active strategies. This pattern has developed in an educational setting where competition is very high and only those with very high grades on exams that require knowledge reproduction and limited application secure medical school admission. 
Looking at the future entrant students expect that things will become more difficult and that more effort will be required. At the same time they expect a shift toward more responsibility and self-direction. They expect that their teachers will enable selfregulation and support their learning by building good rapport with their students and by being approachable. The pattern described here has been graphically represented in Figure 5.1.

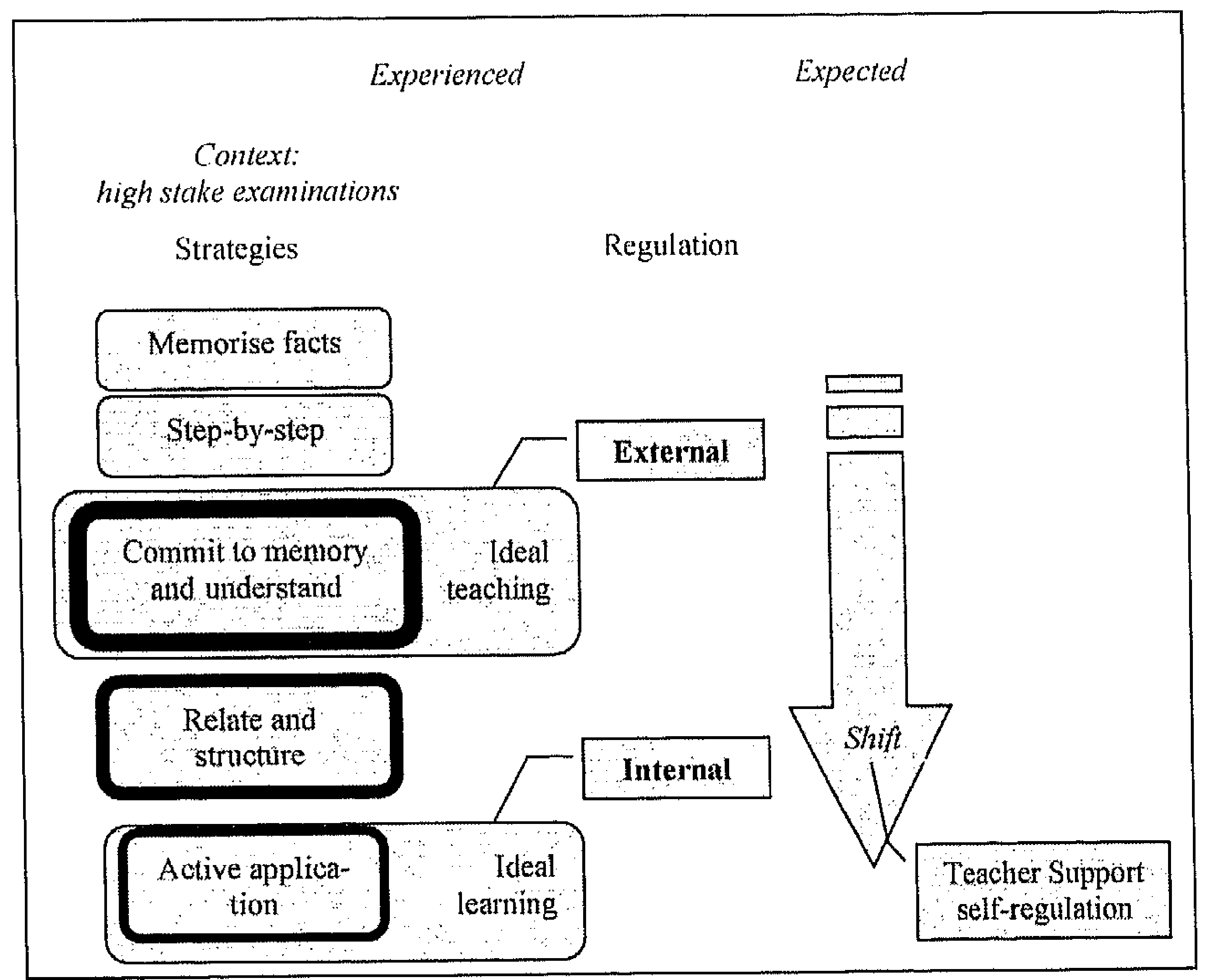

Figure 5.1 Overview of study and regulation strategies and their relation to ideal learning and teaching, and expectations about future learning.

Legend: The thicker the border of the strategy buttons the more students indicated that they used this strategy.

This analysis shows some inconsistencies that may have implications for future learning. One is that the students' ideals and expectations are not supported by their experiences. The majority has shown to come to high performances in high stake situations using a strategy that consists of committing to memory and understand. Their descriptions of ideal learning are based on incidents, not on routines. This implies that the shift that they expect will require the support they hope for, or else they will not be able to make that change. Things are even less likely to happen if they are right that things will become more difficult. Reverting to well known strategies is likely to happen in such situations, especially if examinations will be similar to what students have been accustomed to.

Analysis of generalities and differences revealed that not many distinctive patterns could be identified. Almost all students shared some features. Not only did most students use the strategy of committing to memory and understand, also did most students entertain more or less explicit constructivist believes about knowledge and learning. Less than 
half the students reported learning strategies theoretically in balance with their regulation strategies and with their conceptions of learning. Another remarkable finding was that internal and external regulation seemed not mutually exclusive; a small group used internal and external regulation. This latter group also used deep and surface processing strategies.

Another revelation was that at high school level committing to memory and understanding had been sufficient to meet the examination demands satisfactorily. However a matter of concern is half the students have not indicated the use of deep learning strategies such as relating and structuring information or applying the knowledge in practice for greater understanding of the subject areas. The present results are very much in line with Van Mil's (2005) finding that repetition and memorization is a commonly used learning strategy among the first year Chinese students studying in a Dutch university. In the latter case this strategy was not so successful, since the exams in this university required deep and critical processing. This experience may have instilled a persistent belief that in high stake situations one best revert to this strategy.

The majority of students had a constructivist conception of learning. This finding is more consistent with results of Lonka et al. (1996), who reported a positive relationship between the amount of constructivity in participants' definition and general level of education. All the students except one used a folk notion of knowledge representation; none of them used standard cognitive psychological terminology in explaining learning. This finding is once again not surprising, as Lonka and colleagues reported that, if at all, very few participants other than expert psychology students, used terminology suggestive of mental representations such as "schemata" or "knowledge structures" to define learning.

The majority of the medical students at the beginning of the course seemed to be keen to become doctors and was intrinsically motivated. Most of them indicated that they are from middle-class background parents who are teachers, professionals and skilled workers. It is well known that attitudes and beliefs are strongly influenced by society and the culture one lives in. Sri Lankan students also revealed to have parents who see a doctor as a professional, an important member of society, commanding a position of respect, similar to the situation in the United Kingdom (Rennie, 2000). Some of the students also indicated an altruistic motivation to study medicine, while some others implied that they entered for perceived prestige and power. This hierarchy in motives deviated from those reported by Hilliard (1995). Students of Faculty of Medicine, University of Toronto, rated the importance of some nine motives for entering medical school. Most students indicated that they chose medicine because they wanted to pursue an intellectually challenging career. The other highly rated items were desire to help others and be useful to society, to be good physicians with good clinical skills working with people, whereas a desire to follow a family tradition pressure from family was rated rather low (Hilliard, 1995).

The in-depth interviews with a representative sample of novice medical students were a rich source of data.Unlike most studies, which researched the aspects of learning during university education the current study is unique in the sense it explored the experiences and beliefs of new entrants. A qualitative study of this nature focuses on mechanisms and understanding as opposed to a quantitative study, which enables generalisations. This is a weakness in the eyes of quantitative methodology. However, current study sample was rather large for a qualitative study. Furthermore, since the selection of students to Sri Lankan universities is based on a common criterion and is handled by an apex body (see Chapter 2), the study sample is not only representative of new entrant medical students in Peradeniya, but can also be viewed as revealing study patterns of the new en- 
trant population of all Sri Lankan medical schools. By making such generalizations about a whole country or culture we follow the footsteps of several other researchers (Lonka et al., 1996, Marton et al., 1997; Vermunt, 1996), who have studied the views and beliefs of students on learning components using similar methods and have made similar generalizations on such data.

Vermunt (1998) stated that the learning conceptions and motives influence the study strategies and the regulation of learning: a constructivist conception of learning and intrinsic motivation would be associated with the use of deep learning strategies, while a knowledge assimilation conception and extrinsic motivation would be associated with the use of surface strategies. Although, several studies in western countries, among a range of students, have shown the presumed relation between conceptions, study strategies and regulation strategies, regardless of the method used, some researchers have also observed lack of integration between learning aspects irrespective of the phase of education (Vermunt \& Verloop, 2000). Lindblom-Ylänne and Lonka (2000) also reported disintegration between the learning strategies students used and their conceptions and orientations among high achieving advanced medical students and interpreted the findings as a misfit between learning strategies the learning environment fosters and the strategies students themselves want to use. Similarly, in the current study the reported beliefs were not consistent with the behaviour in the case of most students. Other research with Asian students seems to corroborate this finding (e.g., Ajisuksmo \& Vermunt, 1999; Purdie Hattie, \& Douglas, 1996; Van Mil, 2005). This result may be due to diverse causes: the period of education, research method, educational context and cultural difference.

Two theories have been put forward to explain the phenomenon of disintegration between the learning activities students use and their beliefs on learning and orientations. Based on the results of studies conducted among students in the different phases of secondary and tertiary education, using the ILS as the test instrument, Vermunt and Vermetten (2004) formulated a developmental hypothesis, meaning that with the students' progress in education the associations between beliefs and views on learning, learning orientations and learning strategies become stronger. According to this hypothesis the more mature students are, the higher the internal coherence among learning components. Opposing the former, Severiens (1997) put forward a context hypothesis to explain his findings with adult students using the ILS. Of the two, contextual hypothesis is better suited to explain the phenomenon of disintegration between beliefs on learning and learning activities undertaken by the Sri Lankan medical entrants during the latter part of their secondary education.

The reason why we prefer a contextual explanation of the student behaviour is based on the fact that the target population had experienced the given learning environment for a considerable length of time. It is hard to discern between effects of education and culture, since these two are so interrelated. The study sample almost exclusively comprised very keen, motivated students, who (had) experienced in a very competitive learning environment. Rather than a developmental effect non-congruent conceptions of teaching and learning, study motivation and the stated characteristics of an ideal student could well be due to the influence of this competitive learning environment. Nearly half of students described that their learning was externally controlled and had to be achievement oriented. Since achievement orientation is something created by the environment the highly competitive nature of the selection examination (as described in Chapter 2, the ratio of students admitted to medical school to the number eligible for admission being about 1:12) could be responsible for this difference. An achievement orientation (Biggs, 
1987) or a strategic approach consists of the intent to maximize performance and grades, allocating study time and effort in a systematic deliberate fashion and adopting deep or surface strategies according to what is judged as optimally efficient for attaining grades (Eley, 1992). The reported study strategies of a majority of students seem to conform to a strategic approach (characterized by the syllabus bounded ness and over reliance on external control of learning content and results), irrespective of the stated motives. Within the Sri Lankan education context a strategic approach appears to be a sensible option in order to secure a place in medicine.

Theoretically when students attribute failure to internal, controllable causes, such as lack of effort or insufficient knowledge they tend to focus on strategies for succeeding next time this was amply described by some of the student quotes. This is an adaptive mastery oriented response leading to achievement and a sense of self-determination (Ames, 1992). From the attributions that the medical students made, it was evident that they were aware of their high capabilities: it was a matter of not working hard enough that led to failure; therefore they were determined to make necessary adaptations and succeed next time. In situations where they succeed the success is commonly attributed to internal, controllable causes such as 'hard work or use of effective study strategies'. Therefore, it can be concluded, as it is the case with most medical students (Rennie, 2000), that these students too are highly motivated and are of the achieving type, attributing failures due to lack of effort rather than lack of ability.

Finally the results show that students see themselves as able to self regulate the learning process and that they aspire self directed learning. However, the data also reveal that the secondary school learning context was not geared at promoting active learning among students. University selection test (GCE A/L described in Chapter 2) is criticized for testing lower levels of knowledge assimilation. In explaining why students in the Asian region show a greater propensity to combine memorizing and understanding, Kember (1996) hypothesised that it could be due to linguistic or cultural causes. However, in this particular group of students, since the medium of instruction so far had been their mother tongue, it is reasonable to assume that it is the influence of the learning environment (teaching assessment, restrictions in the education system), which in turn is influenced by the culture that has given rise to the described learning style.

The highly competitive nature of university selection, the teaching methods employed and the overall curriculum can be held responsible for creating this kind of a destructive friction (Vermunt \& Verloop, 1999) in the learning process. As pointed out by Vermunt and Verloop (1999), friction occurs when the teacher takes over learning activities from students that they are capable of using on their own accord and this will ultimately result in a decrease in the use of learning and thinking activities by students. The results also show that these medical students have experienced a strongly teacher controlled senior secondary education system. The students' ideal image of teaching at the university is that it supports learning and enables self-regulation. Thus, the new entrants have the right frame of mind and chances are they will adapt to a student oriented learning environment, provided there is sufficient support during the transition period, something the university teachers seem to doubt.

The next chapters (especially Chapters $6 \& 8$ ) will show whether this optimism that is not built on students' own experience will materialise in the later years. In these chapters the relation between strategies, conceptions and orientations will be investigated in the later years, using an adapted version of the ILS. 


\section{Development of the adapted version of the inventory of learning styles: the Adyayana Rata Prakasha Malawa}

\section{Introduction}

The learning activities that students employ determine to a large extent the quality of the learning outcomes they achieve. Therefore, instruction should be directed at encouraging the use of high quality learning activities. It is often expressed that a goal of higher education is to promote self-regulated learning so that the amount of external control, in which the teacher is taking over the control of learning processes, is minimal (Vermetten, 1999). This means there is greater emphasis on gradual transfer of control over student learning processes from teacher and/or other instructional agents to students (Vermunt \& Verschaffel, 2000). Hence, an upsurge in the interest in describing and measuring the learning or study strategies of students in higher education was witnessed over the last decade. This trend in educational research can be partly attributed to the fact that convincing empirical evidence is increasingly sought to inform policy decisions, some of which may relate to staff development (Entwistle \& McCune, 2004). However, to-date the extent of self-regulation of the learning process among Sri Lankan undergraduate medical students has not been documented. Thus, the studies reported in this chapter are aimed at researching students' reported use of learning activities, their regulation of the learning process, learning orientations and conceptions of learning at the Faculty of Medicine, University of Peradeniya within the existing conventional curriculum.

As described in chapter 3 , a number of researchers has investigated associations between learning inventory scale scores on the one hand and academic performance and personal factors such as age, gender, academic locus of control, and study time of university students on the other (e.g. Ajisuksmo, 1996; Drew \& Watkins, 1998; Severiens \& Ten Dam, 1997; Vermunt, 2005). According to the literature, meaning orientation and its subscales are generally positively related to academic performance, while the reproducing orientation is mostly negatively associated with academic performance (Stiernborg \& Bandaranayake, 1996). Thus, the studies here reported are aimed at identifying the learning patterns of Sri Lankan medical students and the presence or absence of associations of these patterns with various personal and contextual factors. The outcomes of such studies have potential usefulness in educational decision-making.

It is common practice in educational research to study learning processes by administering self-report questionnaires or inventories. These questionnaires are increasingly used to obtain information about the strong and weak sides of students' way of learning. At university level it is important to gather diagnostic information about the learning skills of learners as a group. This could then lead to decisions with regard to the aspects to be trained, separately and/or to be emphasised during regular lessons (Van Hout-Wolters, 2000). At present, many such standardized inventories are available in English, for example, the Inventory of Learning Styles (ILS; Vermunt, 1998), the Study Process Questionnaire (SPQ; Biggs, 1987), the Motivated Strategies for Learning Questionnaire (MSLQ; Pintrich, Smith, Garcia, \& Mckeachie, 1993), the Revised Approaches to Studying Inventory (RASI; Entwistle \& Tait, 1994) and the Learning and Study Strategies Inventory (LASSI; Weinstein, Palmer, \& Schulter, 1987). These questionnaires seem to differ in goal, content, target groups, type of questions/statements, number of scales and items, fill-in time, reliability and validity. However, all of them try to gain insight into learners' 
cognitive and metacognitive learning activities by the learners written retrospection ( $V$ an Hout-Wolters, 2000). There is a trend of construction of new questionnaires on the basis of items of different existing standardised questionnaires as well. In addition, some of the inventories have been translated into other languages and elaborated enabling them to be used in foreign countries (Ajisuksmo, 1996; Albaili, 1997). Ajisuksmo (1996) composed an Indonesian version of the $I L S$, called "Inventarisasi Cara Beljar" to measure learning patterns among Indonesian higher education students.

In general, advantages of using inventories as an assessment instrument over conducting interviews and thinking aloud methods are that the former avoid disturbing learners during studying and that the collection of data and the scoring of the questionnaires take less time. Moreover, inventories minimize subjective bias. However, a frequently quoted disadvantage of this method is that the actual behaviour is not assessed instead, only the students' ideas about it are assessed (Vermetten, Vermunt \& Lodewijks, 1999a). Finally, social desirability could also play a role, and hence, there could be a temptation to provide socially desirable responses (Stiernborg \& Bandaranayake, 1996). Therefore, it is important to acknowledge the limitations of self-report instruments in interpreting results.

\section{Selection of a measuring tool}

Most of the available inventories are developed in western cultures and therefore, they represent aspects of learning described by those students. According to Best and Ruther (1994), various factors in an individual's environment will influence the development of his or her behaviour. Hofstede (2001) described the term "culture" as the collective programming of the mind that distinguishes the members of one group of people from another. The central feature of a particular culture is its value system. As an individual grows in a particular culture he/she invariably acquires these values, rituals and symbols. Symbols are words, gestures, pictures and objects that carry often complex meanings recognized as such only by those who share the culture. Therefore, language is an important component of culture. Thinking is affected by the categories and words available in one's language, equivalents of concepts in another language are sometimes missing and in that case additional words have to be used to convey the meaning (Hofstede, 2001). Hence, when applying an inventory developed in another country to Sri Lankan students, it might be possible that some phenomena as viewed by the Sri Lankan students are missing. Van de Vijver and Poortinga (1991) stated that, among other things, unfamiliarity with testing situations, incompetence in understanding instructions and characteristics of the testing situation might influence the interpretation of tests. Therefore, it was imperative to develop a culturally acceptable inventory.

A diagnostic instrument to measure the different aspects of student learning, namely, cognitive processing activities, metacognitive regulation activities, student motivations and learning conceptions, is not available in the native language of Sri Lanka, i.e. Sinhala. Therefore, as the first step, an instrument had to be developed, based on an existing standardized inventory. Unlike the earlier inventories, the ILS has drawn ideas from metacognition (Flavell, 1987). Metacognition includes ideas and beliefs about learning, which is the static aspect of metacognition, as well as active, 'on line' regulation of learning, including activities as orienting on a learning task, monitoring whether the learning process proceeds as planned, diagnosing the cause of difficulties and adjusting learning process when needed, which represents the dynamic aspect of metacognition. Thus, in the present study the $I L S$ composed by Vermunt (1998) was selected as the basis to develop a 
valid and reliable instrument to measure learning patterns of Sri Lankan undergraduate medical students.

\section{The Inventory of Learning Styles (ILS) and its development}

This instrument has been constructed in the context of a research project on students' regulation of learning processes in higher education in the Netherlands (Vermunt \& Vermetten, 2004). At the time of its development, little was known about regulation of learning activities in the way students use them, the stylistic aspects of regulation of learning, and the way in which regulation is associated with students' use of processing strategies. Vermunt (1998) investigated these interrelations between cognitive and regulative learning strategies, conceptions of learning and learning orientations.

The development of the ILS was based on phenomenographic analyses of interviews with first year regular as well as Open University students about their ways of learning, their ideas about learning, studying and teaching, and their motives, concerns, and personal goals in their studies (Vermunt, 1996). Based on the categories of description that were the result of that phenomenographic study, Vermunt (1998) selected statements from the interviews that were considered to be characteristic for the various categories. When necessary, the formulations have been adapted. These descriptions were included as items in the inventory. In deriving these items, Vermunt (1998) examined the existing inventories as well as the more general literature on student learning. Subsequently, in various studies the final version of the instrument had been constructed using factor, reliability, item, and test-retest analyses. For a description of this construction process and the psychometric qualities of the instrument, see Vermunt (1998).

The first results of the research project were first reported in a book written in Dutch. Later on, the results were discussed at international conferences and the analyses were refined, new analyses were conducted, as well as the theoretical background had been elaborated (e.g., Vermunt, 1995, 1996, 1998; Vermunt \& Verloop, 1999). The ILS has been used extensively by Dutch researchers in studies with university students and students in different stages of secondary education (for example Beishuizen, Stoutjedijk, \& Van Putten, 1994; Beishuizen \& Stoutjesdijk, 1999; Busato, Prins, Elshout, \& Hamaker, 1998; Severiens \& Ten Dam, 1997; Vermetten et al. 1999a, 1999b). Later on, also researchers from other countries, for example, Belgium (Minnaert \& Janssen, 1997; Schatteman, Carette, Couder, \& Eisendrath, 1997), Finland (Lonka \& Lindblom-Ylänne, 1996), Indonesia (Ajisuksmo \& Vermunt, 1999), and Great Britain (Boyle, Duffy, \& Dunleavy, 2003) have used the $I L S$ as a research instrument. Schatteman et al. (1997) administered the ILS to medical and dental students in order to investigate their learning skills. Furthermore, this instrument has also been used as an instructional tool to stimulate students to reflect on, and to develop, their ways of learning at Tilburg University, The Netherlands (Vermunt, 1995).

The final version of the $I L S$ consists of 120 statements that cover four learning components. These components are: (a) cognitive processing strategies, which are the thinking activities used by students to process the subject matter (27 items); (b) metacognitive regulation strategies, that control and regulate the cognitive processing activities (28 items); (c) conceptions of learning, which are the views of learning and knowledge and the student's ideas about the task division between oneself and others in the learning process (40 items); and (d) learning orientations, that cover the area of student motivation, referring to the whole domain of students' personal goals, intentions, attitudes, worries and doubts (25 items) (Vermunt, 1998). The $I L S$ generates 20 scale variables. With 
regard to cognitive processing, five distinctive strategies that students use to process learning content have been identified by principal component analyses. Similarly, five dimensions were discerned for each of the other domains, i.e. regulation strategies, conceptions of learning, and learning orientations. Table 6.1 describes the different components of learning and the meaning of different scales identified by the ILS.

Table 6.1. Description of the scales of the Inventory of Learning styles (ILS), including a sample item for each scale (Source Vermunt \& Vermetten, 2004, p. 365-366.)

\begin{tabular}{|c|c|}
\hline Parts and scales of the $I L S$ & Description of content \\
\hline \multicolumn{2}{|l|}{$\begin{array}{l}\text { Processing strategies } \\
\text { Deep processing }\end{array}$} \\
\hline Rclating \& structuring & $\begin{array}{l}\text { Relating elements of the subject matter to each other and to prior } \\
\text { knowledgc, structuring these elements into a whole. } \\
\text { 'I try to combine the subjects that are dealt with separately in a coursc } \\
\text { into one whole'. }\end{array}$ \\
\hline Critical processing & $\begin{array}{l}\text { Forming one's own view on the subjects that are dealt with, drawing } \\
\text { one s own conclusions, and being critical of the conclusions drawn by } \\
\text { textbook authors and teachers. } \\
\text { "I try to be critical of the internretations of experts". }\end{array}$ \\
\hline \multicolumn{2}{|r|}{ 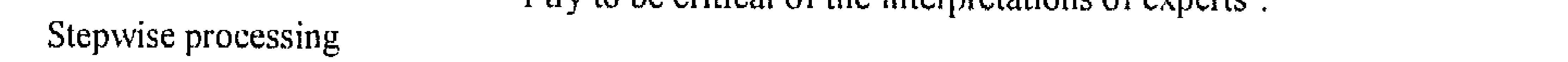 } \\
\hline $\begin{array}{l}\text { Memorizing \& Rehears- } \\
\text { ing }\end{array}$ & $\begin{array}{l}\text { Learning facts, definitions, lists of characteristics and the like by heart } \\
\text { by rehearsing them. } \\
\text { 'I memorise lists of characteristics of a certain phenomenon'. }\end{array}$ \\
\hline Analyzing & $\begin{array}{l}\text { Going through the subject matter in a stepwise fashion and studying } \\
\text { the separate elements thoroughly, in detail and one by one. } \\
\text { "I work through a chapter in a textbook item by item and I study each } \\
\text { part separately'. }\end{array}$ \\
\hline Concrete processing & $\begin{array}{l}\text { Concretizing and applying subject matter by connecting it to one's own } \\
\text { experiences and by using in practice what one learns in a course. } \\
\text { 'I try to interpret event in everyday reality with the help of the knowl- } \\
\text { edge I had acquired in the course'. }\end{array}$ \\
\hline \multicolumn{2}{|l|}{$\begin{array}{l}\text { Regulation strategies } \\
\text { Self regulation }\end{array}$} \\
\hline $\begin{array}{l}\text { Learning process and } \\
\text { Outcomes }\end{array}$ & $\begin{array}{l}\text { Regulating one's own learning processes through regulation activities } \\
\text { like planning learning activities, monitoring progress, diagnosing prob- } \\
\text { lems, lesting one's outcomes, adjusting and reflecting. } \\
\text { 'To test my learning progress, I try to answer questions about the sub- } \\
\text { ject matter which I make up myself'. }\end{array}$ \\
\hline Learning contents & $\begin{array}{l}\text { Consulting literature and sources outside the syllabus. } \\
\text { "I add something to the subject matter from other sources." }\end{array}$ \\
\hline \multicolumn{2}{|l|}{ External regulation } \\
\hline Learning process & $\begin{array}{l}\text { Letting one's own learning processes be regulated by external sources, } \\
\text { such as introductions, learning objectives, directions, questions or as- } \\
\text { signments of teachers or textbooks authors. } \\
\text { 'I study according to the instructions given in the study materials or } \\
\text { provided by the teacher'. }\end{array}$ \\
\hline Learning outcomes & $\begin{array}{l}\text { Testing one's learning results by external means, such as the tests, as- } \\
\text { signments and questions provided. } \\
\text { 'I test my learning progress solely by completing the questions, tasks } \\
\text { and exercises provided by the teacher or the texthook.' }\end{array}$ \\
\hline Lack of regulation & $\begin{array}{l}\text { Monitoring difficulties with the regulation of one's own learning proc- } \\
\text { esses } \\
\text { 'I notice that it is difficult for me to determine whether I have mastered } \\
\text { the subject matter sufficiently'. }\end{array}$ \\
\hline
\end{tabular}


Table 6.1. Continued.

\begin{tabular}{|c|c|}
\hline Parts and scales of the $\mid L S$ & Descriplion of content \\
\hline \multicolumn{2}{|l|}{ Conceptions of learning } \\
\hline Construction of Knowledge & $\begin{array}{l}\text { Learning viewed as constructing one s own knowledge and insights. } \\
\text { Most learning activities are seen as tasks of students. } \\
\text { "If I have difficulty understanding a particular topic, I should consult } \\
\text { other books of my own accord". }\end{array}$ \\
\hline Intake of knowledge & $\begin{array}{l}\text { Learning viewed as taking in knowledge provided by education } \\
\text { through memorizing and reproducing; other learning activities are tasks } \\
\text { of teachers. } \\
\text { 'To me, lcarning is making sure that } 1 \text { can reproduce the facts pre- } \\
\text { sented in the course'. }\end{array}$ \\
\hline Use of knowledge & $\begin{array}{l}\text { Learning viewed as acquiring knowledge that can be used by means of } \\
\text { concretizing and applying. These activities are seen as tasks of both } \\
\text { students and teachers. } \\
\text { 'The things I learn have to be useful for solving practical problems. }\end{array}$ \\
\hline Stimulating cducation & $\begin{array}{l}\text { Learning activities are viewed as tasks of students, but teachers and } \\
\text { textbook authors should continuously stimulate students to use these } \\
\text { activities. } \\
\text { 'The teacher should encourage me to compare the various theories that } \\
\text { are dealt with in a course'. }\end{array}$ \\
\hline Cooperative learning & $\begin{array}{l}\text { Attaching a lot of value to learning in co-operation with fellow stu- } \\
\text { dents and sharing the tasks of learning with them. } \\
\text { "I have a need to work together with other students in my studies". }\end{array}$ \\
\hline \multicolumn{2}{|l|}{ Learning orientations } \\
\hline Personally interested & $\begin{array}{l}\text { Studying out of interest in the course subjects and to develop oneself as } \\
\text { a person. } \\
\text { "I do these studies out of shecr interest in the topics that are dealt with". }\end{array}$ \\
\hline Certificate oriented & $\begin{array}{l}\text { Striving for high study achievement; studying to pass examinations and } \\
\text { to obtain certificates, credit points and a degree. } \\
\text { "The main goal I pursue in my studies is to pass exams. }\end{array}$ \\
\hline Self-test oriented & $\begin{array}{l}\text { Studying to test one's own capabilities and to prove to oneself and oth- } \\
\text { crs that one is able to cope with the demands of higher education. } \\
\text { 'I want to test myself to see whether I am capable of doing studies in } \\
\text { higher education". }\end{array}$ \\
\hline Vocation oriented & $\begin{array}{l}\text { Studying to acquire professional skill and to obtain a(nother) job. } \\
\text { "I have chosen this subject area, because it prepares me for the type of } \\
\text { work I am highly interested in". }\end{array}$ \\
\hline Ambivalent & $\begin{array}{l}\text { A doubtfiul, uncertain attitude toward the studies, one's own capabili- } \\
\text { ties, the chosen subject area, the type of education, etc. } \\
\text { 'I am afraid these studies are too demanding for me'. }\end{array}$ \\
\hline
\end{tabular}

For the strategy items, students are instructed to indicate on a five-point Likert scale the degree to which they use the described learning activities in their studies. The scales vary from (1) I seldom or never do this; to (5) I almost always do this. For the items on conceptions of learning and learning orientations, respondents are instructed to indicate on a five-point scale the degree to which the described views and motives correspond to their own views and motives. In this part the scale varies from (1) completely disagree to (5) completely agree.

Vermunt (1998) reported that internal consistencies of the ILS scales computed for regular university students varied between 0.63 to 0.85 for processing strategies, from 0.48 to 0.79 for regulation strategies, from 0.70 to 0.89 for conceptions of learning, and from 0.57 to 0.84 for learning orientations. He also proposed an integrated model of student learning underlying the ILS where the students' conceptions of learning and learning 
orientations influence the way the students' regulate their learning processes. He conceptualised learning conceptions and learning orientations as more stable, individual variables, and learning strategies as more dynamic, changing aspects of learning.

Research using the $I L S$ has repeatedly identified four learning patterns among first year students in a number of Dutch universities (Vermunt \& Vermetten, 2004). The first factor typically shows high loadings of relating and structuring, concrete processing and critical processing strategies, self-regulation of learning processes and learning contents, construction of knowledge as a conception of learning, and personal interest as learning orientation. This dimension was interpreted as a meaning directed learning pattern or 'learning style'. The second factor was characterised by high loadings of the ILS scales memorising and rehearsing, analysing, external regulation of learning processes and learning outcomes, intake of knowledge as the conception of learning, and certificate and self-test directed learning orientations. This dimension was named a reproduction directed learning pattern or style. The third factor was interpreted as an undirected learning pattern and characterised by high loadings of lack of regulation, an ambivalent learning orientation and co-operation and stimulating education as conceptions of learning. The fourth factor was characterised by high loadings of concrete processing strategies, use of knowledge as conception of learning and a vocational learning orientation, and this was interpreted as an application directed learning pattern.

The $I L S$ checks the study habits students usually employ. However, it was also found that with specific adaptations (such as rewording the instructions to respondents, so that the students were made to reflect on a specific course), the instrument could also be used to measure the ways of studying within a particular context, i.e. during a learning task, course unit or semester (Busato et al., 1998; Vermetten et al., 1999a).

\section{Development of the Sri Lankan version of the Inventory of Learning Styles (ILS): the Adyayana Rata Prakasha Malawa (ARPM)}

Objective. The main objective of studies 2 and 3 , reported in this chapter, was to develop a valid, reliable and practical diagnostic instrument to measure the learning strategies, approaches, orientations and conceptions of Sri Lankan higher education students. Therefore, the focus of the studies was on the construction of the ARPM scales for the Sri Lankan Medical students, and on establishing the internal scale consistencies. Additionally, it was also felt interesting to examine the associations between learning scale scores and personal and performance variables.

Study 2 and study 3. Study 2 describes the process of pilot testing of the Sinhala version of the ILS (ARPM1) among a group of Dental students registered at the Faculty of Dental Sciences, University of Peradeniya. Study 3 refers to the administration of ARPM2 ( $2^{\text {nd }}$ version) and the personal data questionnaire to new entrants, first year students, third year students and fourth year students at the faculty of Medicine, University of Peradeniya. In this chapter, the process of validation of the diagnostic instrument, which is an adapted version of the ILS called the Adyayana Rata Prakasha Malawa, is described in detail. 
Research questions. In summary, the research questions of the present study were seven fold:

1. What is the reliability of the ARPM-1 scales?

2. To what extent can the internal consistencies of scales be increased?

3. Which learning styles of Sri Lankan students can be identified by the ARPM-2?

4. What is the relationship between students' $A R P M$ scale scores and personal factors such as gender, attempt at entry and average amount of independent study time per day?

5. What is the relationship between students' ARPM scale scores and contextual variables such as level of maternal education, parental occupation, district from which the student has secured entry to medical faculty?

6. What is the association between students' ARPM scale scores and academic performance?

7. Is there a relationship between the ARPM scale scores and students' academic locus of control? Does the academic locus of control change among the different student groups?

\section{Study 2}

\section{Introduction}

This pilot study was aimed at constructing the first version of the ARPM and validating its scales. Therefore, this study addressed the accuracy of translation and the feasibility of the data collection technique. The specific research question addressed was: What is the reliability of the ARPM-1 scales?

\section{Method}

Students The ARPM-1 was administered to students newly registered for the undergraduate course of study in the Faculty of Dental Sciences, University of Peradeniya. As the first step permission was sought from the Dean, Faculty of Dental Sciences to contact these students. After getting permission, 50 students were randomly selected for the sample. The participation was voluntary. Thirty-two out of these 50 students replied (response rate $62 \%$ ). About $54 \%$ of the respondents were males.

The ARPM The ARPM is the translated Sri Lankan version of the ILS. The data obtained by interviews with Sri Lankan medical students (described in chapter 5) enabled the researcher to ascertain whether the items of the $I L S$ covered the aspects of learning of Sri Lankan students, and also to include aspects more specific to the Sri Lankan population that were missing in the ILS.

In order to minimize errors in translating the ILS, a group of students from the same batch as those who were interviewed (Chapter 5) was requested to translate the ILS items into Sinhalese. The researcher made use of some of the terminology revealed by the students' during the translation process. The purpose of getting the students to translate the items was to enable the researcher to check whether the students' interpretation of the inventory items was the same as intended by the instrument developers and to revise all items that the students did not understand well. In order to avoid translation errors and misinterpretations of the terminology used in the inventory, the resultant Sinhala version of the inventory was further refined, taking into consideration the comments of two col- 
leagues who were bilingual (both fluent in Sinhala and English languages). The educational background of the translator was considered important in order to avoid translation errors and misinterpretations of terminology used in the inventory. Instances where the exact Sinhala word could not be found or where the English version was vague, the English terminology was presented in brackets as it was thought to help the respondent to understand what was being questioned. Finally, back translations of some of the tricky $A R P M$ - items was done by an independent person, who helped to check whether some of the items had been distorted in the translation process. At the end of the above process the Adyayana Rata Prakasha Malawa - I (ARPM-I), an adapted version of ILS, had been constructed. The $A R P M-1$ contained 123 items. Of the 3 additional items adopted from the interviews, two were included in the "Ambivalent" scale and 1 item was added to the learning conception scale "stimulating education". This adapted translated, 123 item Sinhala version of $I L S$, the $A R P M-1$, was used in study 2.

Procedure The ARPM-1 was posted to all 50 students in the sample, with a covering letter containing all relevant instructions and a paid envelope to return the completed questionnaire. Participation in the study was voluntary and the respondents were in no way rewarded for their participation. A reminder was sent to the non-respondents.

Data Analysis The SPSS procedures were used in the data analysis. Reliability analyses were performed to compute the internal consistencies of the ARPM scales. Mean item means and mean item standard deviations (SDs) of the ARPM scales were calculated.

\section{Results}

In Table 6.2 the Cronbach alpha reliability coefficients of the ARPM-1 scales are shown. Cronbach alpha reliability coefficients range from 0.13 for the scale intake of knowledge, to 0.81 for the scale cooperation. The alpha's for the processing scales range from 0.66 to 0.84 , while the alpha for regulation strategies scales varies from 0.33 to 0.77 , for conceptions of learning from 0.13 to 0.81 and for learning orientations from 0.53 to 0.74 . Two scales were found to have an alpha value lower than .50: intake of knowledge (0.13), and external regulation of process $(0.33)$. When both external regulation subscales were combined into the main scale 'external regulation', the alpha was thought to be acceptable. To compare with, the internal consistencies of the $I L S$ scales ranged from 0.48 for External regulation of process to 0.89 for Co-operation (Vermunt, 1998). 
Table 6.2. Number of items (No.). means (M). standard deviation (SD) and Cronbach alpha coefficients $(\alpha)$ of ARPM-I scales (Faculty of Dental Sciences. University of Peradeniya. Sri Lanka) and ILS scales (Tilburg University, the Netherlands; from Vermunt, 1998. p. 160).

\begin{tabular}{|c|c|c|c|c|c|c|c|c|}
\hline \multirow{2}{*}{ Scales } & \multicolumn{4}{|c|}{$A R P M 1(\mathrm{n}=32)$} & \multicolumn{4}{|c|}{ ILS $\quad(\mathrm{n}=795)$} \\
\hline & No. & M & SD & $\alpha$ & No. & M & SD & $\alpha$ \\
\hline \multicolumn{9}{|l|}{ Processing strategies } \\
\hline Deep processing & 11 & 3.32 & .81 & .84 & 11 & 3.16 & 1.21 & .85 \\
\hline Relating \& structuring & 7 & 3.50 & .79 & .75 & 7 & 3.36 & 1.18 & .83 \\
\hline Critical processing & 4 & 3.00 & 1.03 & .69 & 4 & 2.81 & 1.25 & .72 \\
\hline Stepwise proccssing & 11 & 3.15 & .67 & .66 & 11 & 2.78 & 1.29 & .78 \\
\hline Memorizing \& rehearsing & 5 & 2.63 & .98 & .72 & 5 & 2.83 & 1.30 & .79 \\
\hline Analyzing & 6 & 3.59 & .81 & .66 & 6 & 2.73 & 1.16 & .63 \\
\hline Concrete processing & 5 & 3.34 & .79 & .71 & 5 & 2.81 & 1.17 & .71 \\
\hline \multicolumn{9}{|l|}{ Regulation strategies } \\
\hline Self-regulation & 11 & 3.50 & .72 & .79 & 11 & 2.30 & 1.19 & .79 \\
\hline $\begin{array}{l}\text { Learning process \& out- } \\
\text { comes }\end{array}$ & 7 & 3.56 & .87 & .77 & 7 & 2.54 & 1.28 & .73 \\
\hline L earning contents & 4 & 3.58 & .86 & .58 & 4 & 1.87 & 1.03 & .73 \\
\hline External regulation & 11 & 2.97 & .54 & .58 & 11 & 3.22 & 1.22 & .68 \\
\hline Learning process & 6 & 2.67 & .55 & .33 & 6 & 3.08 & 1.21 & .48 \\
\hline Learning outcomes & 5 & 3.34 & .87 & .64 & 5 & 3.38 & 1.23 & .65 \\
\hline Lack of regulation & 6 & 2.16 & .93 & .76 & 6 & 2.40 & 1.17 & .72 \\
\hline \multicolumn{9}{|l|}{ Conceptions of learning } \\
\hline Construction of knowledge & 9 & 4.11 & .43 & .65 & 9 & 3.53 & 1.10 & .78 \\
\hline Intake of knowledge & 9 & 3.71 & .91 & .13 & 9 & 3.52 & 0.99 & .77 \\
\hline Use of knowledge & 6 & 4.19 & .40 & .50 & 6 & 3.91 & 0.91 & .70 \\
\hline Stimulating education & 9 & 3.83 & .57 & .79 & 8 & 3.13 & 1.13 & .88 \\
\hline Cooperation & 8 & 3.67 & .71 & .81 & 8 & 3.01 & 1.20 & .89 \\
\hline \multicolumn{9}{|l|}{ Learning orientations } \\
\hline Personally interested & 5 & 4.14 & .61 & .62 & 5 & 3.17 & 1.04 & .57 \\
\hline Certificate oriented & 5 & 3.32 & .62 & .53 & 5 & 3.28 & 1.18 & .76 \\
\hline Self-test oriented & 5 & 3.35 & .77 & .54 & 5 & 2.83 & 1.28 & .84 \\
\hline Vocation oriented & 5 & 4.01 & .64 & .59 & 5 & 3.79 & 1.07 & .69 \\
\hline Ambivalent & 7 & 2.23 & .79 & .74 & 5 & 2.07 & 1.12 & .82 \\
\hline
\end{tabular}

\section{Study 3}

\section{Development of ARPM 2}

The Cronbach alpha reliability coefficients of two scales in the ARPM 1 were found to be lower than 0.50 . It was decided to add 7 items to these two scales, in order to improve their reliability: four items to the scale intake of knowledge and three items to the scale use of knowledge. Moreover, the items of the scale external regulation of outcomes, and the instructions to the students were further clarified. Thus, the revised version of the $A R P M$, the $A R P M-2$, contained 130 items: 55 items under processing and regulating strategies, 27 items under learning orientations and 48 items under conceptions of learning. Processing and regulating items were scored on five-point Likert scales, with scores ranging from (1) 'I never or hardly ever did this' to (5) 'I almost always did this', while 
the latter items are scored on five point Likert scale with scores ranging from (1) completely disagree to (5) completely agree.

\section{Method}

Students The ARPM 2 was administered to the new entrants, medical students in the first year of study, third and fourth years of study at the Faculty of Medicine, Peradeniya separately in the year 2000. In the respective batches, all students present in the lecture room (see below) completed the questionnaire and these were collected in the lecture theatre itself. Hence, comparatively high response rates could be obtained: more than $80 \%$ for every batch of students. For the background characteristics of the students, see Table 6.3.

Materials The ARPM 2 was administered along with a questionnaire to gather personal information of each student. It was hypothesised that the scale scores are related to variables such as gender, and maternal occupation (see Chapter 3). Personal data were necessary to achieve some of the objectives of the study. A data form was designed to obtain information on:

1. Student registration number (for identification purposes)

2. Age in years

3. Gender

4. The G.C.E. A/L (university selection examination, described in chapter 2) aggregate at the last successful attempt (the attempt in which the student was selected for Medicine),

5. The attempt at which medical school admission was secured $(1,2$ or 3$)$

6. District from which the student secured admission to Faculty of Medicine

7. Average number of hours devoted to independent studies per day

8. The highest level of maternal education (completed primary education only, successfully completed senior secondary education, university education etc.)

9. Type of maternal and paternal employment (teacher or non teacher).

10. Attribution patterns of students during an academic failure. The open question asked was: "Think of a situation where you have not performed as expected in relation to your studies. How do you attribute the failure in such a situation?"

In Table 6.3 the values these variables could take are shown, together with the frequencies and percentages of these values in the actual sample.

Performance data (e.g. A/L aggregate score and respective examination scores) were obtained from the student records in the Dean's office and the Faculty mark book with the permission of the Vice-Chancellor of the University.

Procedure Medical students of the 1999/2000 batch were contacted, and time from the English course was taken with the permission of the coordinator English language Teaching Unit to administer the ARPM 2 and the personal data questionnaire. The majority of the students completed the Sinhala inventory while a few students responded to the English version, as they were not very fluent in Sinhala. The ARPM 2 was administered to the medical students in the first year of study, third year and fourth years of study separately, but in a similar way. In the above instances arrangements were made with the respective 
Heads of Departments to utilize one hour of their lecture time for the purpose of administering the inventory.

Data analyses Reliability analyses were performed using SPSS procedures. Internal consistencies, Cronbach alpha $(\alpha)$, were computed for the 20 different scales of the inventory for the whole group of students, and for each group separately. Mean and SD for the ARPM scales were computed using excel and SPSS programmes for the different groups of students. Factor analysis was executed to examine the relationships between the ARPM-variables for the whole group of students. Principal component analysis with Varimax rotation was also executed in the SPSS program for identification of factors over all four batches. Furthermore, for each student factor scores were computed using the Minitab statistical package.

The attribution pattern of the students was analyzed after coding the student answers on the basis of Weiner's model of attribution into exclusive categories, as described in Table 5.2 of Chapter 5. With respect to locus of control the data were categorized into three separate variables: a) internal (1) or external (2); b) controllable (1) and uncontrollable (2); c) unstable (1) and stable (2).

The other variables were coded as follows:

1. Age in years - the age of students was recoded as follows: 18-20 years as (1), 21-23 years (2), 24-26 years ( 3 ), > 26 years (4).

2. Gender - to make this categorical variable suitable for data analysis it was coded into 1 and 2.

3. The GCE A/L aggregate score was recoded as $<225$ (1), 225-250 (2), 251-275 (3), 276-300 (4), 301-325 (5), >326 (6).

4. District from which student secured university entry - to make this categorical variable suitable for data analysis it was recoded into two groups as 1 and 2 (privileged / underprivileged respectively).

5. The attempt at which university entry was secured remained as $1,2,3$.

6 . The average number of hours devoted for independent study per day was also recoded as less than 2 hrs (1), 2-3 hrs (2), 3-4 hrs (3), and > 4 hrs (4).

7. Highest level of maternal education was recoded into 2 categories: (1) successfully completed senior secondary education and (2) less than senior secondary level.

8. Maternal and paternal employment data were recoded as (1) teacher and (2) nonteacher.

9. First, third and fourth year students' academic performance scores during the MBBS course of study were recoded into categories: $31-50$ marks as (1), 51-70 marks as (2) and $71-90$ marks as (3).

Relationships between ARPM-variables and personal, contextual, and performance variables were computed using the statistical techniques described in Table 6.4 below. The SPSS statistical package was used to conduct analysis of variance, correlation coefficients and independent sample t-tests. Regression analysis was conducted to identify relationships between the academic performance data and factor scores using Statistical Analysis System (SAS).

\section{Results}

In Table 6.3 the values of the 9 background variables are shown. The student sample mainly comprised of those aged $24-26$ years (47\%) and $21-23$ years (46\%). The sample 
showed a slight male preponderance. More than half the students reported an Advanced Level aggregate of 276-300 out of 400 marks. A majority of them has studied in an educationally privileged area $(83 \%)$. It appeared that most of the students have succeeded in their $2^{\text {nd }}$ attempt to enter Medicine.

Table 6.3. Frequencies $(\mathrm{N})$ and percentages (\%) of the values on personal, contexlual and performance variables for the total group of students in study 3.

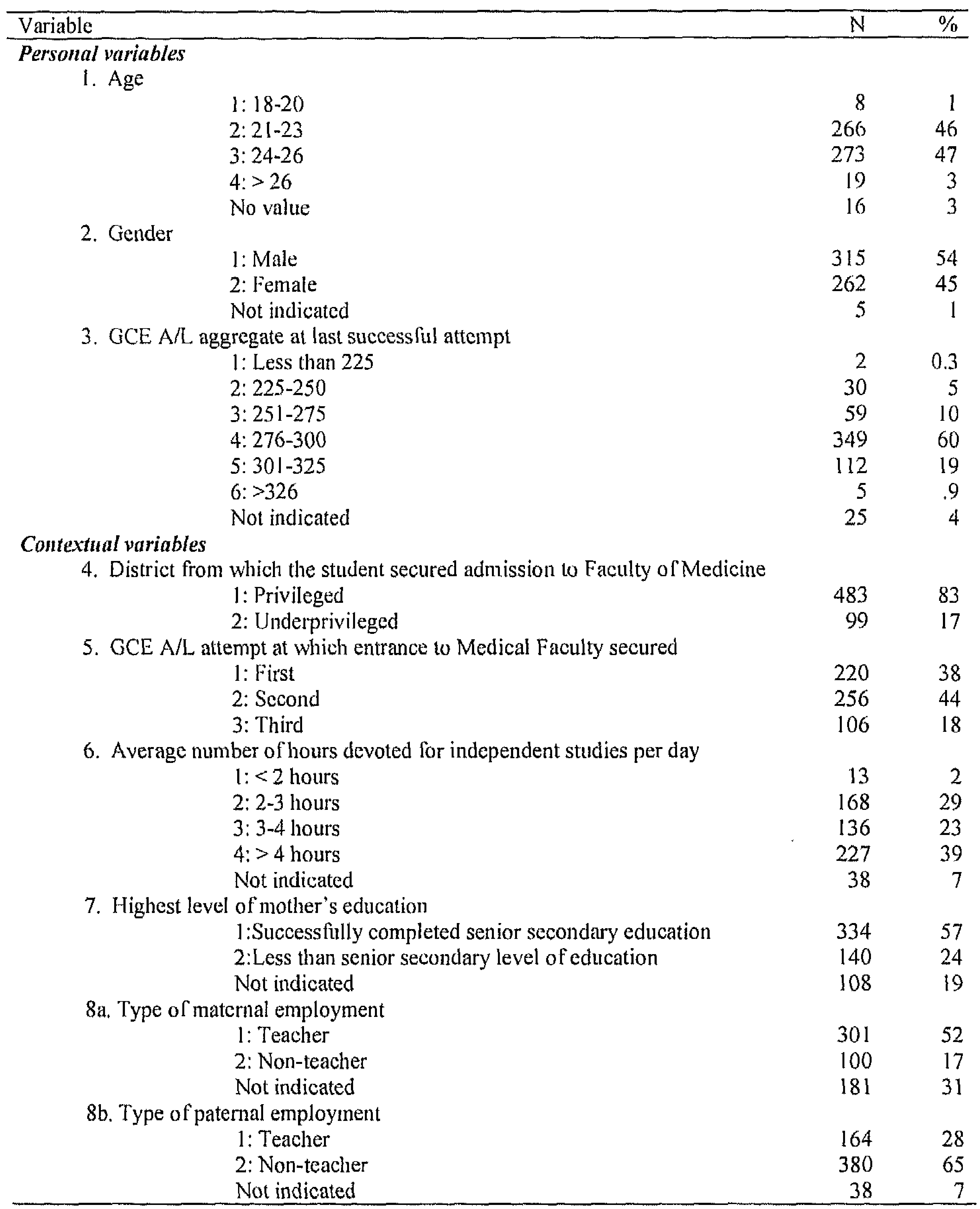


Table 6.3. Continued.

\begin{tabular}{crr}
\hline Variable & $\mathrm{N}$ & $\%$ \\
\hline 9. Locus of control & 483 & 83 \\
1: Internal & 454 & 78 \\
2: Controllable & 518 & 89 \\
3: Unstable & & \\
Performance variable & & \\
10.Academic performance - percentage of scores obtained at the end of the & & 12 \\
corresponding course & 51 & 12 \\
1:31-50 & 365 & 86 \\
$2: 51-70$ & 8 & 2 \\
$3: 71-90$ &
\end{tabular}

A majority indicated that they spend more than 4 hours per day on an average for studies. A majority stated that their mother is a teacher (57\%). Most of the students reported that their mothers are reasonably educated. As was expected a vast majority reported an internal, controllable and unstable academic locus of control. Most of the students ( $86 \%)$ have obtained scores in the range of 51-70 marks in the respective examinations.

Table 6.4 summarizes what data analysis techniques were conducted on what variable pairs.

Table 6.4. Variable pairs and the corresponding data analyses techniques.

\begin{tabular}{|c|c|c|}
\hline Background variables and values & Learning style variables & Statistical technique \\
\hline \multicolumn{3}{|l|}{$\overline{\text { Personal Variables }}$} \\
\hline 1. Age & ARPM scale scores & Analysis of variance \\
\hline 2. Gender & Factor scores & Independent sample t test \\
\hline $\begin{array}{l}\text { 3. GCE A/L aggregatc at last successful } \\
\text { attempt }\end{array}$ & ARPM Scale scores & Analysis of variance \\
\hline \multicolumn{3}{|l|}{ Contextual Variables } \\
\hline 4. District of entry to medical school & ARPM scale scores & Independent sample t test \\
\hline $\begin{array}{l}\text { 5. GCE A/L attempt at which entrance } \\
\text { to medical faculty secured }\end{array}$ & Factor scores & Analysis of variance \\
\hline $\begin{array}{l}\text { 6. Average number of hours devoted for } \\
\text { independent studies per day }\end{array}$ & ARPM scale scores & $\begin{array}{l}\text { Spearman's rank correla- } \\
\text { tion test }\end{array}$ \\
\hline 7. Highest level of mothers' education & ARPM scale scores & Independent sample t test \\
\hline 8a.Type of maternal employment & ARPM scale scores & Independent sample I test \\
\hline 8b.Type of paternal employment & ARPM scale scorcs & Independent sample t test \\
\hline 9. Locus of control & Factor scores & $\begin{array}{l}\text { Independent sample t test } \\
\text { carried out for internal vs } \\
\text { external, controllable vs } \\
\text { uncontrollable and stable } \\
\text { vs unstable separately }\end{array}$ \\
\hline \multicolumn{3}{|l|}{ Performance variable } \\
\hline 10. Academic performance & $\begin{array}{l}\text { ARPM scale scores and } \\
\text { factor scores }\end{array}$ & $\begin{array}{l}\text { Correlation and regres- } \\
\text { sion analyses }\end{array}$ \\
\hline
\end{tabular}

In Table 6.5 the number of items, means, standard deviations, and Cronbach alpha $(\alpha)$ of the ARPM-2 scales are presented for the total group of students. 
Table 6.5. Number of items per scale (No.), means (M), standard deviation (SD) and Cronbach alphas $(\alpha)$ of ARPM-2 scales. for the lotal number of students $(N=582)$.

\begin{tabular}{lcccc}
\hline ARPM-2 scales & No. & M & SD & $\alpha$ \\
\hline Processing strategies & 11 & 2.91 & 0.63 & .84 \\
Deep processing & 7 & 3.24 & 0.80 & .76 \\
$\quad$ Relating \& Structuring & 4 & 2.58 & 0.94 & .72 \\
$\quad$ Critical processing & 11 & 2.80 & 0.52 & .64 \\
Stepwise processing & 5 & 2.53 & 0.84 & .64 \\
$\quad$ Memorizing \& Rehearsing & 6 & 3.16 & 0.73 & .64 \\
$\quad$ Analyzing & 5 & 3.30 & 0.86 & .76 \\
Concrete processing & & & & \\
& & & & \\
Regulationt strategies & 11 & 3.00 & 0.70 & .77 \\
Sclf-regulation & 7 & 3.02 & 0.79 & .72 \\
$\quad$ Lcarning process & 4 & 2.88 & 0.91 & .60 \\
$\quad$ comes & 11 & 3.00 & 0.60 & .66 \\
Learning contents & 6 & 2.74 & 0.66 & .50 \\
External regulation & 5 & 3.38 & 0.83 & .63 \\
$\quad$ Learning process & 6 & 2.81 & 0.80 & .62 \\
$\quad$ Learning outcomes & & & & \\
Lack of regulation & & & & \\
& 9 & 3.94 & 0.52 & .71 \\
Conceptions of learning & 13 & 3.67 & 0.49 & .63 \\
Construction of knowledge & 9 & 4.20 & 0.49 & .72 \\
Intake of knowledge & 9 & 3.80 & 0.76 & .71 \\
Use of knowledge & 8 & 3.74 & 0.81 & .78 \\
Stimulating education & & & & \\
Cooperation & & & & \\
Learning orientations & 5 & 3.48 & 0.65 & .58 \\
Personally interested & 5 & 3.34 & 0.74 & .56 \\
Certificate oriented & 5 & 3.39 & 0.72 & .52 \\
Self-lest oriented & 5 & 4.10 & 0.69 & .40 \\
Vocation oriented & 7 & 2.74 & 0.77 & .68 \\
Ambivalent & & & & \\
\hline
\end{tabular}

In Table 6.6 these statistics are presented for the four batches separately. For the total group of students, the internal consistencies (Cronbach alpha) of the ARPM-2 scales range from 0.41 for the scale self-test oriented (being the lowest) to 0.87 for the cooperation scale (being the highest).

Table 6.7 shows the factor loadings resulting from principal component analysis with Varimax rotation over all four batches. Four factors were extracted for theoretical and statistical reasons. Furthermore, Eigen values of the first four factors were higher than 1 . The factor analyses were also conducted for the four batches separately, but these analyses yielded highly similar results. Therefore, here only the results of the overall analysis will be reported.

The first factor (FI) with high loadings of relating and structuring, critical processing, concrete processing, self regulation of learning processes and learning contents, construction of knowledge as conception of learning and personal interest as learning orientation is similar to meaning oriented learning pattern. However, analysing, although a stepwise processing strategy, also loads high on this factor among the Sri Lankan students. The second factor (F2) has high loadings of the processing scale memorizing and rehears- 
ing, external regulation of learning processes and outcomes, and moderate loadings of certificate oriented and self-test directed orientations and the conception intake of knowledge. The third factor (F3) can be viewed as passive idealistic learning pattern with high loadings of all the conceptions of learning. High loadings of lack of regulation, ambivalent orientation and a negative loading of self-regulation and personally interested learning orientation characterize factor 4.

Table 6.6. Internal consistencies (Cronbach alpha) of $A R P M-2$ scales of the four different batches of students.

\begin{tabular}{|c|c|c|c|c|}
\hline ARPM Scale & $\begin{array}{c}\text { New entrant } \\
\mathrm{N}=159\end{array}$ & $\begin{array}{c}\text { First year } \\
N=144\end{array}$ & $\begin{array}{c}\text { Third year } \\
\mathrm{N}=143\end{array}$ & $\begin{array}{c}\text { Fourth year } \\
N=136\end{array}$ \\
\hline \multicolumn{5}{|l|}{ Processing strategies } \\
\hline Deep processing & .81 & .83 & .83 & .88 \\
\hline Relating \& Structuring & .70 & .75 & .76 & .83 \\
\hline Critical processing & .68 & .73 & .70 & .76 \\
\hline Stepwise processing & .58 & .65 & .67 & .66 \\
\hline Memorizing \& Rehearsing & .64 & .56 & .61 & .65 \\
\hline Analyzing & .54 & .60 & .65 & .67 \\
\hline Concrete processing & .68 & .77 & .80 & .82 \\
\hline \multicolumn{5}{|l|}{ Regulation strategies } \\
\hline Self-regulation & .74 & .73 & .79 & .77 \\
\hline $\begin{array}{l}\text { Learning process and out- } \\
\text { comes }\end{array}$ & .62 & .68 & .73 & .71 \\
\hline Learning contents & .70 & .50 & .58 & .59 \\
\hline External regulation & .63 & .69 & .68 & .67 \\
\hline Learning process & .47 & .49 & .45 & .50 \\
\hline Learning outcomes & .60 & .60 & .60 & .59 \\
\hline Lack of regulation & .57 & .66 & .54 & .59 \\
\hline \multicolumn{5}{|l|}{ Conceptions of learning } \\
\hline Construction of knowledge & .65 & .73 & .64 & .69 \\
\hline Intake of knowledge & .67 & .66 & .65 & .62 \\
\hline Use of knowledge & .67 & .74 & .69 & .64 \\
\hline Stimulating education & .85 & .66 & .79 & .83 \\
\hline Cooperation & .80 & .67 & .81 & .87 \\
\hline \multicolumn{5}{|l|}{ Learning orientations } \\
\hline Personally interested & .60 & .55 & .63 & .50 \\
\hline Certificale oriented & .58 & .63 & .51 & .53 \\
\hline Self-test oriented & .41 & .58 & .55 & .54 \\
\hline Vocation oriented & .70 & .50 & .48 & .56 \\
\hline Ambivalent & .70 & .68 & .59 & .66 \\
\hline
\end{tabular}


Chapter 6

Table 6.7. Factor loadings of ARPM-2 scales in a four factor Varimax solution for the total group of students ( $\mathrm{N}=582$ : principal component analysis: loadings between -.25 and .25 omitted).

\begin{tabular}{|c|c|c|c|c|}
\hline ARPM -2 scale & $\mathrm{Fl}$ & $\mathrm{F} 2$ & $F 3$ & F4 \\
\hline \multicolumn{5}{|l|}{ Processing strategies } \\
\hline \multicolumn{5}{|l|}{ Deep processing } \\
\hline Relating \& Structuring & .85 & & & \\
\hline Critical processing & .80 & & & \\
\hline \multicolumn{5}{|l|}{ Stepwise processing } \\
\hline Memorizing \& Rehearsing & & .65 & & \\
\hline Analyzing & .72 & .29 & & \\
\hline Concrete processing & .67 & & & \\
\hline \multicolumn{5}{|l|}{ Regulation strategies } \\
\hline \multicolumn{5}{|l|}{ Self-regulation } \\
\hline $\begin{array}{l}\text { Learning process and out- } \\
\text { comes }\end{array}$ & .76 & & & \\
\hline Learning contents & .61 & & & -.29 \\
\hline \multicolumn{5}{|l|}{ External regulation } \\
\hline Learning process & & .72 & & \\
\hline Learning outcomes & .27 & .63 & & \\
\hline Lack of regulation & & & & .71 \\
\hline \multicolumn{5}{|l|}{ Conceptions of learning } \\
\hline Construction of knowledge & .43 & & .68 & \\
\hline Intake of knowledge & & .52 & .53 & \\
\hline Use of knowledge & .29 & & .75 & \\
\hline Stimulating education & & & .74 & \\
\hline Cooperation & & & .59 & \\
\hline \multicolumn{5}{|l|}{ Learning orientations } \\
\hline Personally interested & .45 & & & -.56 \\
\hline Certificate oriented & & .61 & & .28 \\
\hline Self-test oriented & & .46 & .27 & .27 \\
\hline Vocation oriented & & .42 & .39 & -.27 \\
\hline Ambivalent & & & & .80 \\
\hline Eigen valuc & 4.01 & 2.57 & 2.55 & 1.87 \\
\hline$\%$ of variance & 20.05 & 12.86 & 12.77 & 9.37 \\
\hline Cumulative \% & 20.05 & 32.91 & 45.68 & 55.05 \\
\hline
\end{tabular}

The correlation between $A R P M 2$ scales and average independent study time for the total group of students is shown in Table 6.8. The results indicate that the independent study time is positively correlated with the ARPM scales, relating and structuring, memorizing and rehearsing, self-regulation of learning process, self-regulation of contents, external regulation of process, external regulation of outcomes, certificate directed learning orientation, construction of knowledge and intake of knowledge. 
Table 6.8. Significant correlations between average number of hours spent on independent study per day and the $A R P M$ scale scores ( $\mathrm{N}=582$; Spearman's correlation test).

\begin{tabular}{|c|c|}
\hline ARPM-2 Scale & Spearman's correlation coefficient \\
\hline \multicolumn{2}{|l|}{ Processing strategies } \\
\hline \multicolumn{2}{|l|}{ Deep processing } \\
\hline Relating \& Structuring & $.30 * *$ \\
\hline \multicolumn{2}{|l|}{ Critical processing } \\
\hline \multicolumn{2}{|l|}{ Stepwise processing } \\
\hline Memorizing \& Rehearsing & $.30^{* * *}$ \\
\hline \multicolumn{2}{|l|}{ Analyzing } \\
\hline \multicolumn{2}{|l|}{ Concrete processing } \\
\hline \multicolumn{2}{|l|}{ Regulation strategies } \\
\hline \multicolumn{2}{|l|}{ Self-regulation } \\
\hline Learning process and outcomes & $.23 * *$ \\
\hline Learning contents & $.30 * *$ \\
\hline \multicolumn{2}{|l|}{ External regulation } \\
\hline Learning process & $.30 * *$ \\
\hline Learning outcomes & $.21^{* * *}$ \\
\hline \multicolumn{2}{|l|}{ Lack of regulation } \\
\hline \multicolumn{2}{|l|}{ Conceptions of learning } \\
\hline Construction of knowledge & $.18^{*}$ \\
\hline Intake of knowledge & $.27 * *$ \\
\hline \multicolumn{2}{|l|}{ Use of knowledge } \\
\hline \multicolumn{2}{|l|}{ Stimulating education } \\
\hline \multicolumn{2}{|l|}{ Cooperation } \\
\hline \multicolumn{2}{|l|}{ Learning orientations } \\
\hline \multicolumn{2}{|l|}{ Personally interested } \\
\hline Certificate oriented & $.18^{*}$ \\
\hline \multicolumn{2}{|l|}{ Self-test oriented } \\
\hline \multicolumn{2}{|l|}{ Vocation oriented } \\
\hline Ambivalent & \\
\hline
\end{tabular}

In Table 6.9 the locus of control of students is presented. This indicates that the majority of the participant students make internal, controllable and unstable attributions when they fail. According to the results of the cross sectional study the percentage of the sample that reported an internal locus of control at the commencement of the course (85\%), first and the third years $(87 \%)$ were more or less similar whereas the fourth year students $(76 \%)$ have shown a downward trend. The percentages of fourth year students who have attributed academic failure due to controllable and unstable factors have also shown a downward trend.

Table 6.9. Percentages of students indicating academic locus of control as internal, controllable and unstable respectively (coded using Weiner"s model of attribution.)

\begin{tabular}{lccc}
\hline \multirow{2}{*}{ Batch } & \multicolumn{3}{c}{ Locus of control (\%) } \\
\cline { 2 - 4 } & Internal & Controllable & Unstable \\
\hline New entrant & 85 & 82 & 97 \\
First year & 84 & 80 & 94 \\
Third year & 87 & 84 & 96 \\
Fourth year & 76 & 65 & 75 \\
\hline
\end{tabular}


As depicted in Table 6.10 the relationships between personal and contextual variables such as age, gender, attempt at which the student gained entry to the faculty of Medicine, district of entry, parental education and occupation academic locus of control and the learning style scores or the $A R P M$ scale scores for the total group of students (new entrants, first year, third year and fourth year students) were not significant. Although previous studies have revealed associations between inventory scale scores and academic performance the present study failed to reveal similar associations. The only exception is that the reproduction oriented learning style was found to be inversely proportional to academic performance for the fourth year batch of undergraduate medical students.

Table 6.10. Relations between personal, contextual and performance variables and learning styles (factor scores) or $A R P M$ scale scores.

\begin{tabular}{|c|c|c|}
\hline Background variables and values & Learning style variables & Significance $(p<0.05)$ \\
\hline \multicolumn{3}{|l|}{ Personal variables } \\
\hline 1. Age & ARPM scale scores & Not significant \\
\hline 2. Gender (male/female) & Factor scores & Not significant \\
\hline $\begin{array}{l}\text { 3. GCE A/L aggregate at last success- } \\
\text { ful attempt }\end{array}$ & ARPM scale scores & Not significant \\
\hline \multicolumn{3}{|l|}{ Contextual variables } \\
\hline 4. District of entry to medical school & $A R P M$ scale scores & Not significant \\
\hline $\begin{array}{l}\text { 5. GCE A/L Attempt at which the stu- } \\
\text { dent secured entry to the medical } \\
\text { faculty }\end{array}$ & ARPM scale scores & Not significant \\
\hline $\begin{array}{l}\text { 6. Average number of hours devoted } \\
\text { for independent studies per day }\end{array}$ & $A R P M$ scale scores & Refer Table 6.8 \\
\hline 7. Highest level of mothers education & ARPM scale scores & Not significant \\
\hline 8a Type of maternal employment & $A R P M$ scale scores & Not significant \\
\hline 8b Type of paternal employment & $A R P M$ scale scores & Not significant \\
\hline 9. Locus of control & Factor scores & Not significant \\
\hline \multicolumn{3}{|l|}{ Performance variables } \\
\hline 10. Academic performance & $\begin{array}{l}\text { 1. ARPM scale scores, } \\
\text { and } \\
\text { 2. Factor scores }\end{array}$ & $\begin{array}{l}\text { 1. Corrclation - Not significant } \\
\text { 2. Regression analyses for the } \\
\text { fourth-year batch: } \\
A v=49.2+(36.3 * 1 / 43) \\
R^{2}=0.04\end{array}$ \\
\hline
\end{tabular}

Note: $\mathfrak{f}$ in the regression analysis is the reproduction oriented factor score.

\section{Conclusions and discussion}

What is the reliability of the ARPM-1 scales? To what extent can the internal consistencies of scales be increased?

As shown by the results of Study 2, most of the ARPM 1 scales had acceptable internal consistencies that were comparable to those reported by others (Ajisuksmo \& Vermunt, 1999). When the two external regulation scales were combined, the reliability increased. With further adaptation ARPM 2 was developed and this modified version was administered in study 3. With the addition of items to the conceptions of learning scales, intake of knowledge and use of knowledge, the alpha value increased considerably. The results of study 3 indicated that for most of the inventory scales the reliability coefficient was com- 
parable to those reported in the Dutch studies (Vermunt \& Vermetten, 2004). These values are considered acceptable. Previous studies using the $I L S$ or adapted version of the ILS have reported Cronbach alpha values within this range i.e. 0.38-0.78 (Ajisuksmo, 1996), 0.48-0.89 (Vermunt, 1998) and 0.57-0.93 (Vermetten et al. 1999a).

Thus, it is shown that adding items derived from the Sri Lankan interviews could improve the reliability of the ARPM scales. It is concluded that the ARPM 2 is a reliable instrument to assess the learning components among Sri Lankan students. However, the reliability coefficients of the orientation scales remained to be on the low side compared to those of the Dutch study. This is a finding consistent with the Indonesian study (Ajisuksmo \& Vermunt, 1999). In both cultures (Sri Lankan and Indonesian) parents and family members influence the students in their career choices. Thus, the relatively low reliability could be due to the fact that some students begin to think seriously about their orientations and their interests only at this stage and may be confused.

\section{Which learning styles of Sri Lankan students can be identified by ARPM- 2?}

Four learning patterns (learning styles) were identified among Sri Lankan medical students. Previous studies have shown that extracting four factors on ILS subscales is appropriate (Busato et al., 1998; Severiens \& Ten Dam, 1997; Vermetten et al., 1999a; Vermunt, 1998; Vermunt \& Vermetten, 2004). From the study two general types of learning styles could be discerned. Based on the processing and regulation strategies that students usually use, one is called actively directed i.e. this is characterized by actively processing and directing learning activities. The second is a passive learning style, which was characterized by passive processing and incapability in directing learning activities. Furthermore, these two general types of learning styles can be subdivided into four learning styles similar to the Indonesian experience (Ajisuksmo, 1996), i.e. (1) active meaning directed learning style; (2) an active reproductive learning style, (3). a passive idealistic learning style, and (4) passive-undirected learning style.

The first factor of the Sri Lankan study is characterized by high loadings of four processing strategies, relating and structuring, critical processing, analyzing and concrete processing, and the two self-regulation strategies. Besides, there are moderate loadings of personally interested study orientation and the conception of learning in which construction of knowledge is emphasized on this factor. Thus, it is appropriate to name this dimension as a self regulated and meaning directed learning style. High and moderate loadings for the second factor are from the memorizing and rehearsing scale, the two external regulation scales, intake of knowledge and three of the learning orientation scales: certificate oriented, self-test oriented and vocation oriented. Hence, the second factor can be interpreted as externally regulated and reproductive learning style. The third factor is characterized by high and moderate loadings almost exclusively of the conception of learning scales. Thus, this factor resembles a passive idealistic learning style. The fourth factor is characterized by high loadings of the scales lack of regulation and an ambivalent orientation, and moderate inverse loading of the personally interested study orientation. It is reasonable to interpret this factor as an undirected learning style. The four factors identified in the Sri Lankan study have many similarities with those that resulted from the Indonesian study (Ajisuksmo, 1996). Moreover, both Asian studies did not identify an application directed factor as was seen in several of the Dutch studies, particularly with the advanced undergraduate students (Vermunt, 1998). Instead, a passive idealistic factor with high loadings of all the conceptions of learning was repeatedly observed. The simi- 
larities and differences of the Sri Lankan, Dutch and the Indonesian studies will be discussed more extensively in Chapter 7.

What is the relationship between students' ARPM scale scores and personal factors such as age, gender, attempt at entry and average amount of independent study time per day?

The present study failed to reveal a significant association between age and ARPM scale scores or gender and the learning style scores. This finding is inconsistent with the results of Severiens and Ten Dam's (1997) study, which found that on an average, men scored higher than women on undirected learning and women scored higher than men on reproduction directed learning.

Although studies in the past have reported that the attempt at which university selection was secured has a bearing on the performance of an undergraduate in comparison to variables such as entrance examination scores or the intelligence quotient (Senanayake \& Weerasinghe, 1996), there was no association between the attempt and ARPM scores in this study.

Some ARPM scale scores turned out to be associated with reported independent study time per day. Relating and structuring and memorizing and rehearsing tended to be positively related to study time, but the correlation coefficients were small. Other positive relations were observed in the areas of self-regulation of learning contents, external regulation of learning process, certificate directed learning orientation, and the conception of learning as intake of knowledge. On the basis of these results it can be argued that students with a reproduction oriented learning style spend a reasonable amount of time on independent studying. However, those who engage in relating and structuring type of processing activities and those who attempt to self regulate their learning process were also found to be spending a considerable length of time on independent studying.

What are the relationships between students' ARPM scale scores and contextual variables such as level of parental education/occupation, district from which the student has secured entry to medical faculty?

Here again no statistically significant difference in the mean scale scores was found between those whose mothers have successfully completed senior secondary education and those who have not completed their senior secondary education successfully. In this respect Ajisuksmo (1996) reported a significant interaction effect between metacognitive training and the mother's education for students in the Faculty of Economics.

Independent sample $\mathrm{t}$ test did not yield a statistically significant difference between those whose mothers were teachers and non-teachers for the different learning strategy scale scores. This was contrary to the popular belief among some academics that students whose mothers are teachers employ qualitatively better learning strategies. As expected a significant difference in the inventory scale scores was also not evident among those whose fathers were teachers and others.

No statistically significant difference was observed between the two groups of students who have entered the medical faculty from the so-called educationally disadvantaged districts (e.g. Nuwara Eliya, Badulla, etc.) and educationally advantaged districts (e.g. Kandy, Kurunegala etc.) (see Chapter 2). Based on the results of this study one could argue that the two groups of medical students (those who enter from privileged areas and underprivileged areas) are not diverse in terms of the reported use of learning strategies. 
Thus, contrary to the expectation contribution of the area of schooling was not found to be significant on the development of learning strategies or conceptions of learning.

\section{What is the association between ARPM scale scores and academic per- formance?}

Results of the present study did not reveal significant relationship between the ARPM scale scores and the corresponding examination percentage marks of the course. These results are not consistent with other studies among medical students in which meaning orientation and its subscales were positively related to academic performance and reproducing orientation were negatively related to academic performance (Arnold \& Feighny, 1995, Leiden, Crosby, \& Folmer, 1990, Lindblom-Ylänne \& Lonka, 1999; Stiernborg \& Bandaranayake, 1996). Vermunt and Vermetten (2004), in reviewing the literature, furthermore stated that studies with higher education students in other disciplines have also reported reproduction directed learning in general to show negative correlations with exam results, meaning directed learning to show positive correlations with most indicators for exam results, and undirected learning to be consistently and negatively related to exam results (see also Busato et al., 1998). Nevertheless, regression analysis showed that for the fourth year Sri Lankan medical students, performance, estimated by the average mark obtained at the relevant MBBS examination, was inversely proportional to the reproduction oriented learning factor score. There was no evidence to suggest that other factors were related to the examination performance. One possible explanation (though not the ideal) for the lack of association between meaning orientation or its sub scales and academic performance is that examination for medical students test knowledge which requires limited deep-level processing (Newble, Entwistle, Heika, Jolley, \& Whelan, 1988).

Is there a relationship between the ARPM scale scores and students' locus of control?

What is the academic locus of control of medical students?

The present study failed to reveal a relationship between the locus of control and the learning style among the Sri Lankan students. Furthermore, the results of the present study were not consistent with those of Drew and Watkins (1998) in respect of the relationships between causal attributions and learning approaches. Drew and Watkins (1998) reported that internal locus of control has a direct negative effect on the surface approach to studying. They argued that a student who has an internal locus of control believes that he has control over his own learning and is less likely to resort to superficial learning strategies.

This study indicates that the majority of the participant undergraduate medical students make internal, controllable attributions when they fail. It is said that when students see themselves as capable and attribute failure due to lack of effort or insufficient knowledge, which are controllable causes, the tendency is that such students usually focus on strategies for succeeding next time (Woolfolk, 1998). This is an adaptive, masteryoriented response, one that often leads to achievement and a feeling of control and selfdetermination. Hence, motivation to learn is encouraged. Watkins (1989) reported that perceived personal control or acceptance of personal responsibility for learning is found to be associated with higher academic performance among Filipino students. This study 
provides evidence for Biggs' (1991) argument that Asian students' drive for high achievement is related to the cultural bias for attributing success or failure due to effort.

It is reasonable to state that Sri Lankan medical students, like the Chinese tertiary students tend to attribute their performance to internal, controllable causes such as effort and study skill. This effort attribution is thought to make them more adaptive as it protects the student's self esteem and reduces the chance of learned helplessness in failure situations (Drew \& Watkins, 1998). However, it is also evident that in the fourth year of the course the motivational patterns have changed. Students in the fourth year indicated a downward trend in attributing academic failure due to controllable causes. It seems that feeling in control of one's own learning is related to choosing more difficult academic tasks, putting out more effort and persisting longer in study activities (Weiner, 1994). Moreover, internal locus of control is related to deep level learning which is more likely to lead to higher quality of learning outcome (Watkins \& Astilla, 1984; Drew \& Watkins, 1998). In the present study due to some reason or other the controllability dimension is decreasing among fourth year students. This should be a matter of concern to the faculty, as this trend of decreased controllability has a negative impact on exertion of effort and persistence in learning task during the course of studies (Brunning, Shraw, \& Ronning, 1995). 


\section{A cross-cultural comparison of student learning patterns}

\section{Introduction}

A growing interest in the study of effects of culture on student learning was observed in the past decade. As pointed out by Hofstede (2001) education and culture are interrelated thus; the latter is an influencing factor of student learning behaviour. Several researchers have carried out cross-cultural and qualitative studies to better understand the study habits and conceptions of learning among students from different cultural backgrounds (see Chapter 3). According to literature attempts to memorise, combined with high achievement, has been widely attributed to Asian students (Kember, 1996). However, results of qualitative studies with Australian and Japanese students indicate the need to re-examine some of the widely held beliefs about cross-cultural differences in student learning (Purdie, Hattie, \& Douglas, 1996). Therefore, a cross cultural comparison of learning patterns (learning styles) as well as study approaches, and perceptions of learning of a group of Sri Lankan higher education students that have been identified by the ILS or one of its versions was thought to enrich the present beliefs on adoption of learning behaviour. The present study is a meta-analysis, which aims at clarifying some of the currently held beliefs on student learning approaches.

\section{Cultural differences in approaches to learning}

There is general consensus in research literature in higher education that students exhibit a number of different approaches to learning. As stated in Chapter 3 qualitative and quantitative studies have confirmed a broad distinction between deep and surface approaches to learning (Laurillard, 1997; Morgan, Taylor, \& Gibbs; 1982; Ramsden, 1997; Richardson, 1994; Van Rossum \& Schenk, 1984; Watkins, 1983). Other studies have shown that the important distinction between these two approaches lies in the intention, meaning the presence or absence of intention to understand (Biggs, 1987; Entwistle, 1997; Kember, 1996; Richardson, 1994). The results of studies with Asian learners, particularly with the Chinese, point towards the existence of a 'narrow' approach, which is characterized both by the intention to understand as well as memorise (see Chapter 3). Research with Chinese students has also shown that the dividing line for them does not fall between memorization and understanding but between mechanical memorization and memorization to assist development in meaning (Kember \& Gow, 1990; Marton, Dall' Alba, \& Tse, 1992). Vermunt and Verloop (2000) also raised the possibility of there being cultural differences in the interrelations between learning components. Subsequently, Vermunt and Vermetten (2004) observed that aspects of learning patterns the Dutch experience as separate could well go together among Indonesian students.

This Asian learning pattern has often been referred to as 'the Asian paradox' (Kember, 1996). A phrase that reveals the strong expectations that had emanated from the first findings on learning pattern in western societies. Those patterns nicely accommodated bipolar constructs such as surface - deep, internal - extemal, intrinsic - extrinsic. Yet, analysis of other cultural differences between western and Asian cultures suggests that patterns of learning styles and conceptions might be connected to the power distance, uncertainty avoidance, collectivism and masculinity of the local cultures. 


\section{Culture specific behaviours}

Conceptual equivalence refers to the degree to which the constructs that are measured are the same in different cultures (Poortinga, 1989). As a measure of conceptual equivalence Watkins and Akande (1994) used the similarity of factor structures in two cultures. 'Emics' and 'etics' are abstract constructs used to explain culture specific and universal phenomena, respectively. Emics are culture specific concepts; they apply in a particular culture and no priori claim is made that they apply in another. Etics are culture invariant concepts or universals, if not universal. Those apply to more than one culture (Ho \& $W u$, 2001). In cross-cultural research emic and etic approaches may be combined so that emic ways of measuring constructs are developed and validated. An example of this approach is the three-step approach suggested by Berry (1990). That is, first, existing descriptive categories and concepts are applied tentatively as an imposed etic. Then these are modified so that they represent an adequate emic description from within each system. Finally, shared categories can then be used to build up new categories valid for both systems as a derived etic, which can be expanded if desired, until they constitute a universal. Thus, emically defined constructs can be used in making cross-cultural comparisons (Ho \& Wu, 2001). It is often difficult to find instruments to measure a particular behaviour across cultures. Indeed, it is questionable to assume cross-cultural equivalence of the conceptualization of what an instrument purports to measure. In this regard, the Inventory of Learning Styles (ILS) and adapted translated versions of the ILS, the ICB and the ARPM are known to be valid instruments for assessing learning strategy use in the above three cultures.

\section{Family of instruments}

The Inventory of Learning styles (ILS) (Vermunt, 1994), The Inventarisasi Cara Belajar (ICB) (Ajisuksmo, 1996) and the Adyayana Rata Prakasha Malawa (ARPM) (described in Chapter 6) belong to a family of inventories that explore the interrelationships of the cognitive, metacognitive aspects of learning, conceptions of learning and learning orientations. The latter two are adapted, validated native language versions of the ILS. The ILS has been used to explore the learning styles of first year Dutch University students in a number of studies (Vermunt \& Vermetten, 2004), while the ICB has explored the learning styles of first year Indonesian university students in the fields of management, accountancy, law, business administration, electrical engineering and mechanical engineering (Ajisuksmo, 1996). Subsequently, the ARPM was used to explore the learning styles and learning aspects of Sri Lankan medical students in the Faculty of Medicine University of Peradeniya. It has been shown that the ILS, which was developed in the Netherlands, could be adequately adapted to identify leaning styles of Indonesian students (Ajisuksmo \& Vermunt, 1999). Similarly in Chapter 6 of this thesis, it was also shown that the ILS could be adapted to identify the learning styles of Sri Lankan tertiary students. As a result of adaptations some of the scales differ; nevertheless, many of the scales contain identical items. Therefore, interrelations among learning components in the three studies and the scale scores of the three countries could be compared.

\section{Internal structure of learning styles among first year Dutch University students using the ILS}

The ILS has been administered to 795 first-year regular university students in the faculties of law, economics, sociology, psychology, language and literature etc. in the Nether- 
lands. Mean age of these students was reported as 22.5 years while $56 \%$ were males and $44 \%$ were females (Vermunt, 1998). In the Dutch study mailed back responses were considered. Participation had been voluntary. Results of the varimax-rotated factor solution showed four factors (Table 7.4). The first factor was characterized by high loadings of the processing strategies, relating and structuring, concrete processing and critical processing strategies, self-regulation of learning processes and learning results, personal interest as learning orientation and construction of knowledge as a learning conception. Thus, factor one was interpreted as meaning directed learning pattern. The second factor represents a reproduction- directed learning style, with high loadings of the ILS scales, memorising and rehearsing, analysing, external regulation of learning processes and learning results, intake of knowledge as the learning conception and certificate directed learning orientations. The third factor characterized by high loadings of lack of regulation, an ambivalent learning orientation and co-operation and stimulating education as learning conception is termed undirected style. Finally, the fourth dimension represents an application directed style with high loadings of concrete processing, use of knowledge as the learning conception (mental model of learning) and a vocational learning orientation (Vermunt, 1998).

\section{Internal structure of learning styles among first year Indonesian Univer-} sity students using the ICB

The ICB1 was administered to 888 first year students, in the fields of management, accountancy, law, business administration, electrical engineering and mechanical engineering of the Atma Jaya University in Indonesia (Ajisuksmo \& Vermunt, 1999). The inventory had been completed during regular lecture time, thus students were obliged to participate. However, they have not been rewarded for their participation. Results of the varimax-rotated factor solution had shown four factors (Table 7.4). The first factor of the Indonesian study is characterized by high loadings of all five processing strategies and the two self-regulation strategies and by moderate loadings of the two external regulation strategies and the learning conception in which construction of knowledge is emphasized. This dimension was identified as the self-regulated and meaning-directed learning pattern or style. The second factor characterized by loadings on memorizing and rehearsing, external regulation results and process scales the learning conception, intake of knowledge, learning orientation, certificate directed and moderate loadings on self-test directed, personally interested and vocation directed scales. The second factor was identified as reproduction directed learning style. The third factor with high or moderately high loadings almost exclusively on all five learning conceptions was termed passive idealistic style. Fourth factor is characterized by high loadings of lack of regulation and an ambivalent learning orientation and a moderate inverse loading of vocation oriented orientation and thus resembled an undirected learning style.

\section{Internal structure of Learning styles of Sri Lankan medical students identi- fied with the ARPM}

As described in Chapter 6 four learning patterns (learning styles) were identified among Sri Lankan medical students using an adapted version of the ILS, the ARPM2 (Table 7.4). The first factor of the Sri Lankan study is characterized by high loadings of four processing strategies, relating and structuring, critical processing, analyzing and concrete processing, and the two self-regulation strategies. Besides, there are moderate loadings of personally interested study orientation and the conception of learning in which construction 
of knowledge is emphasized on this factor. This dimension is named as a self-regulated and meaning-directed learning style. High and moderate loadings for the second factor, which is termed externally regulated and reproductive learning style, are from the memorizing and rehearsing scale, the two external regulation scales, intake of knowledge and three of the learning orientation scales: certificate oriented, self-test oriented and vocation oriented. The third factor termed passive idealistic learning style is characterized by high and moderate loadings almost exclusively of conception of learning scales. The fourth factor interpreted as an undirected learning style is characterized by high loadings of the scales lack of regulation and an ambivalent orientation, and moderate inverse loading of the personally interested study orientation.

\section{Goals of the present study}

These three studies provide us with an excellent opportunity to investigate cross-cultural differences between two Asian cultures and one western. Thus, the present meta-analysis of the Sri Lankan study and two other large scale studies (Dutch and the Indonesian) aims at examining similarities and differences in the reported use of learning strategies, learning conceptions and learning orientations, factor structures and the presence or absence of the phenomenon of dissonance among three culturally different groups of first year university students.

More specifically the following three research questions will be investigated:

1. Do Sri Lankan students differ from Dutch and Indonesian students in terms of the reported use of study strategies, orientations of learning and conceptions of learning as assessed by the ARPM, ILS and ICB respectively?

2. Are there similarities and differences in the factor structure of learning patterns identified by the ILS, ICB and the ARPM among first year Dutch, Indonesian and Sri Lankan University students?

3. Are learning patterns discerned within the first year of university education theoretically congruent among the first year Sri Lankan medical students and the first year Dutch and Indonesian students?

\section{Methodology}

A meta-analysis was performed of the results of the studies done by Vermunt (1998), in the Netherlands, Ajisuksmo and Vermunt (1999) in Indonesia, and the study reported in Chapter 6.

In the Dutch study the ILS has been administered to 795 first-year regular university students studying law, economy, econometry, sociology, psychology, language and literature, management information sciences and philosophy in the Netherlands. Mean age of these students was reported as 22.5 years; $56 \%$ were males and $44 \%$ were females (Vermunt, 1998).

In the Indonesian study the ICB1 was administered to 888 first year students, in the fields of management, accountancy, law, business administration, electrical engineering and mechanical engineering of the Atma Jaya University in Indonesia (Ajisuksmo \& Vermunt, 1999).

In the Sri Lankan study the ARPM was administered to a group of 144 first year medical students (response rate of $82 \%$ ) in the Faculty of Medicine, University of 
Peradeniya, Sri Lanka. Mean age of the group of students was 23 years; $51 \%$ were males and $49 \%$ were females

All three studies investigated learning styles and beliefs of first year university students and used a validated native version of the same questionnaire, the ILS. Since the scoring keys for the respective inventory scales were similar, mean scores for the questionnaires as a whole and on subscales can be compared. Comparison of the internal consistency values of the three instruments shows that the internal consistencies of most of the scales of the ICBI are quite high and comparable to those of the ILS (Table 7.1).

Table 7.1. Comparison of the internal consistencies (Cronbach alpha values) of Sri Lankan (ARPM2), Dutch and Indonesian students (Dutch ILS and Indonesian ICBI results, adopted from Vermunt, 1998 and Ajisuksmo, 1996. respectively).

\begin{tabular}{|c|c|c|c|c|c|c|}
\hline Scales & $\begin{array}{c}\text { ILS } \\
N=795\end{array}$ & $\begin{array}{c}\text { Number of } \\
\text { items }\end{array}$ & $\begin{array}{c}\mathrm{ICB1} \\
\mathrm{N}=888\end{array}$ & $\begin{array}{c}\text { Number of } \\
\text { ltems }\end{array}$ & $\begin{array}{l}\text { ARPM2 } \\
\mathrm{N}=144\end{array}$ & $\begin{array}{l}\text { Number of } \\
\text { Items }\end{array}$ \\
\hline \multicolumn{7}{|l|}{ Processing strategies } \\
\hline Deep processing & .85 & 11 & .83 & 11 & .83 & 11 \\
\hline Relating \& structuring & .83 & 7 & .76 & 7 & .75 & 7 \\
\hline Critical processing & .72 & 4 & .69 & 4 & .73 & 4 \\
\hline Stepwise processing & .78 & 11 & .73 & 11 & .65 & 11 \\
\hline Memorizing \& rehearsing & .79 & 5 & .58 & 5 & .56 & 5 \\
\hline Analyzing & .63 & 6 & .62 & 6 & .60 & 6 \\
\hline Concrete processing & .71 & 5 & .64 & 5 & .77 & 5 \\
\hline \multicolumn{7}{|l|}{ Regulation strategies } \\
\hline Self-regulation & .79 & 11 & .78 & 11 & .73 & 11 \\
\hline $\begin{array}{l}\text { Learning process \& out- } \\
\text { comes }\end{array}$ & .73 & 7 & .74 & 7 & .68 & 7 \\
\hline Learning contents & .73 & 4 & .68 & 4 & .50 & 4 \\
\hline External regulation & .68 & 11 & .68 & 11 & .69 & 11 \\
\hline Learning process & .48 & 6 & .68 & 6 & .49 & 6 \\
\hline Learning outcomes & .65 & 5 & .59 & 5 & .69 & 5 \\
\hline Lack of regulation & .72 & 6 & .61 & 6 & .66 & 6 \\
\hline \multicolumn{7}{|l|}{ Conceptions of learning } \\
\hline Construction of knowledge & .77 & 9 & .53 & 9 & .73 & 9 \\
\hline Intake of knowledge & .78 & 9 & .74 & 9 & .66 & 13 \\
\hline Use of knowledge & .70 & 6 & .66 & 6 & .74 & 9 \\
\hline Stimulating education & .88 & 8 & .82 & 8 & .66 & 9 \\
\hline Cooperation & .89 & 8 & .67 & 8 & .67 & 8 \\
\hline \multicolumn{7}{|l|}{ Learning orientations } \\
\hline Personally interested & .57 & 5 & .22 & 5 & .55 & 5 \\
\hline Certilicate oriented & .76 & 5 & .62 & 5 & .63 & 5 \\
\hline Self-test oriented & .84 & 5 & .55 & 5 & .58 & 5 \\
\hline Vocation oriented & .69 & 5 & .46 & 5 & .50 & 5 \\
\hline Ambivalent & .82 & 5 & .64 & 5 & .68 & 7 \\
\hline
\end{tabular}

Only in the domain of learning orientations (more specifically, personally interested and vocation oriented) are these internal consistencies generally lower than those obtained with Dutch students. Similarly, internal consistencies of most of the scales of the ARPM2 are high and comparable to findings with the ILS. Although the scale external regulation of process showed a low alpha value, the value of external regulation scale was acceptable. The vocation directed scale of the ARPM2 showed a moderately low value (.50). 


\section{Data analyses}

Differences between Sri Lankan and Dutch students, and between Sri Lankan and Indonesian students in terms of the reported use of study strategies, orientations of learning and conceptions of learning were investigated by performing two-tailed t-tests on subscale means. Such an investigation was possible because the minimum and maximum scores for inventory items irrespective of its version were 1 and 5 respectively.

The $p$-level was adjusted using Bonferoni correction by dividing $p=.05$ by the number of comparisons made. This led to a $p$-value of 0.003 . Similarities and differences in the factor structures of learning patterns were investigated in the following way. For each sample principal component analysis followed by varimax rotation had been performed to extract four factors. These extracted factor structures of the three samples were compared by contrasting high positive and negative loadings on these factors. Special attention was given to deviations from the Dutch factor pattern, since this pattern can be considered theoretical congruent.

\section{Results}

Comparing the sub-scale findings in the Dutch and the Sri Lanka studies the following was found. Three of the five processing strategies were significantly different. Students in the Sri Lankan study more often reported to use concrete processing (Table 7.2 ).

Table 7.2. Comparison of the mean scale scores of the first year Sri Lankan medical students $(N=144)$ and the first year Dutch students ( $N=795$, adopted from Vermunt, 1998) using the two-sample $t$ test.

\begin{tabular}{|c|c|c|c|}
\hline Inventory scales & $\begin{array}{c}\text { Sri Lankan }(N=144) \\
\text { Mean (SD) }\end{array}$ & $\begin{array}{c}\text { Dutch }(\mathrm{N}=795) \\
\text { Mean }(\mathrm{SD})\end{array}$ & $1(937))$ \\
\hline \multicolumn{4}{|l|}{ Processing strategies } \\
\hline Relating \& Structuring & $3.17(0.77)$ & $3.36(1.18)$ & -1.86 \\
\hline Critical processing & $2.32(0.87)$ & $2.81(1.25)$ & $-4.51^{*}$ \\
\hline Memorizing \& Rehearsing & $2.41(0.73)$ & $2.83(1.30)$ & $-3.77^{*}$ \\
\hline Analyzing & $3.02(0.68)$ & $2.73(1.16)$ & 2.91 \\
\hline Concrete processing & $3.19(0.87)$ & $2.81(1.17)$ & $3.72 *$ \\
\hline \multicolumn{4}{|l|}{ Regulation strategies } \\
\hline Self regulation & $2.75(0.67)$ & $2.30(1.19)$ & $4.41 *$ \\
\hline External regulation & $3.11(0.60)$ & $3.22(1.22)$ & -1.06 \\
\hline Lack of regulation & $2.84(0.79)$ & $2.40(1.17)$ & $4.34^{*}$ \\
\hline \multicolumn{4}{|l|}{ Conceptions of learning } \\
\hline Construction of knowledge & $3.93(0.50)$ & $3.53(1.10)$ & $4.28 *$ \\
\hline Intake of knowledge & $3.74(0.45)$ & $3.52(0.99)$ & 2.62 \\
\hline Use of knowledge & $4.16(0.47)$ & $3.91(0.91)$ & $3.22 *$ \\
\hline Stimulating education & $3.80(0.77)$ & $3.13(1.13)$ & $6.83^{*}$ \\
\hline Co-operation & $3.85(0.91)$ & $3.01(1.20)$ & $7.99^{*}$ \\
\hline \multicolumn{4}{|l|}{ Learning orientations } \\
\hline Personally interested & $3.44(0.63)$ & $3.17(1.04)$ & $3.02^{*}$ \\
\hline Certilicate oriented & $3.39(1.18)$ & $3.28(1.18)$ & 1.03 \\
\hline Self-test oriented & $3.41(0.72)$ & $2.83(1.28)$ & $5.29^{*}$ \\
\hline Vocation oriented & $4.10(0.79)$ & $3.79(1.07)$ & $3.32 *$ \\
\hline Ambivalent & $2.84(0.77)$ & $2.07(1.12)$ & $7.92 *$ \\
\hline
\end{tabular}


At the same time memorising and rehearsing was reported less often by these students. The same was true for the critical processing strategy, which represents a meaningdirected learning pattern. Among the regulation strategies, Sri Lankan students reported the use of self-regulation more often but at the same time expressed lack of regulation more often. The mean scores of all the orientations except that of certificate directed orientation were significantly higher than those reported by the Dutch students. Furthermore, Sri Lankan students showed significantly higher scores for all five conceptions of learning, except for intake of knowledge.

As depicted in Table 7.3 the mean scale scores between Sri Lankan and the Indonesian students differed significantly for only two of the five processing scales. The Sri Lankan students more often reported the use of relating and structuring while the Indonesian students more often used memorising and rehearsing strategies. No difference was found on any of the regulation scales. On the learning orientation scales, Sri Lankan students showed to be less certificate oriented, less self-test oriented and less vocationoriented. On the conceptions of learning scales, Indonesian students more often expressed beliefs of construction of knowledge, intake of knowledge and use of knowledge; Sri Lankans endorsed stimulating education significantly more.

Table 7.3. Comparison of the mean scale scores of the first year Sri Lankan medical students $(N=144)$ and the first year Indonesian students ( $N=888$, adopted from Ajisuksmo, 1996) and results of the two-samples $t$ test.

\begin{tabular}{|c|c|c|c|}
\hline Inventory scales & $\begin{array}{c}\text { Sri Lankan }(N=144) \\
\text { Mean (SD) }\end{array}$ & $\begin{array}{c}\text { Indonesian }(\mathrm{N}=888) \\
\text { Mean }(\mathrm{SD}) \\
\end{array}$ & $\mathrm{t}(1030)$ \\
\hline \multicolumn{4}{|l|}{ Processing strategies } \\
\hline Relating \& Structuring & $3.17(0.77)$ & $2.56(1.61)$ & $4.46^{*}$ \\
\hline Critical processing & $2.32(0.87)$ & $2.18(1.34)$ & 1.21 \\
\hline $\begin{array}{l}\text { Memorizing \& Rehears- } \\
\text { ing }\end{array}$ & $2.41(0.73)$ & $3.33(1.69)$ & $-6.43^{*}$ \\
\hline Analyzing & $3.02(0.68)$ & $2.92(1.57)$ & 0.75 \\
\hline Concrete processing & $3.19(0.87)$ & $2.99(1.37)$ & 1.69 \\
\hline \multicolumn{4}{|l|}{ Regulation strategies } \\
\hline Self regulation & $2.75(0.67)$ & $2.76(1.67)$ & -0.07 \\
\hline External regulation & $3.11(0.60)$ & $3.19(1.53)$ & -0.62 \\
\hline Lack of regulation & $2.84(0.79)$ & $2.65(1.36)$ & 1.63 \\
\hline \multicolumn{4}{|l|}{ Conceptions of learning } \\
\hline $\begin{array}{l}\text { Construction of knowl- } \\
\text { edge }\end{array}$ & $3.93(0.50)$ & $4.12(0.86)$ & -2.58 \\
\hline Intake of knowledge & $3.74(0.45)$ & $4.14(1.04)$ & $-4.55^{*}$ \\
\hline Use of knowledge & $4.16(0.47)$ & $4.57(0.46)$ & $-9.89 *$ \\
\hline Stimulating education & $3.80(0.77)$ & $3.17(1.45)$ & $5.09^{*}$ \\
\hline Cooperation & $3.85(0.91)$ & $3.82(1.12)$ & 0.31 \\
\hline \multicolumn{4}{|l|}{ Learning orientations } \\
\hline Personally interested & $3.44(0.63)$ & $3.33(1.30)$ & 0.99 \\
\hline Certificate oriented & $3.39(1.18)$ & $3.96(1.23)$ & $-5.19^{*}$ \\
\hline Self-test oriented & $3.41(0.72)$ & $4.12(1.09)$ & $-7.56^{*}$ \\
\hline Vocation oriented & $4.10(0.79)$ & $4.43(0.75)$ & $-4.86^{*}$ \\
\hline Ambivalent & $2.84(0.77)$ & $2.79(1.82)$ & 0.32 \\
\hline
\end{tabular}


Chapter 7

Table 7.4 was constructed to test the similarities and differences in the factor structures of learning patterns between the first year Dutch, Sri Lankan and Indonesian university students, respectively.

Table 7.4. Comparison of the results of a four-factor principal component analysis with varimax rotation of Dutch first year students using the ILS (N=795); adopted from Vermunt, 1998, Indoncsian first year students using the ICB1 ( $N=888$; adopted from Ajisuksmo \& Vermunt, 1999) and those of first year Sri Lankan medical students using the ARPM $2(\mathrm{~N}=144)$. [*NL- Netherlands, Dutch, SL - Sri Lanka, IN Indonesia]

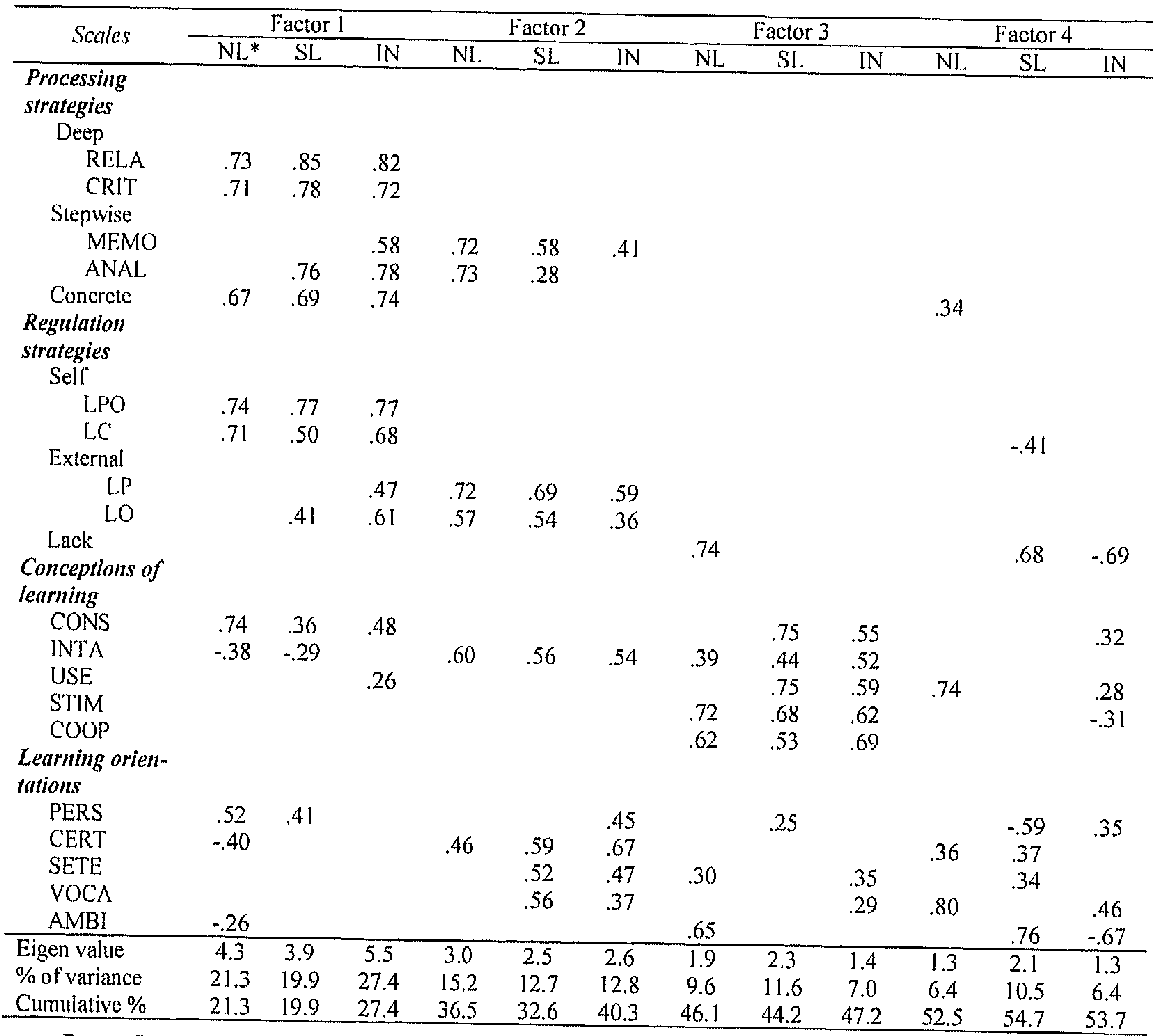

Deep - Deep processing, RELA - Relating \& structuring, CRIT - Critically processing, MEMO - Memorizing and rehearsing, ANAL- Analyzing, Concrete - Concrete processing, Self - Self regulation, LPO Learning process \& outcomes, LC - Learning contents, External - External regulation, LP - Learning process, LO - Learning outcomes, Lack - Lack of regulation. CONS - Construction of knowledge. INTA - Intake of knowledge, USE - use of knowledge, STIM - Stimulating education, COOP - Cooperation, PERS - Personally interested, CERT - Certificate oriented, SETE - Self test oriented, VOCA - Vocation oriented, and $\mathrm{ABMI}-$ Ambivalent 
The similarities and differences will be described per factor. Factor 1 in all three studies turned out to have large similarities. All marking variables with high loadings were shared, although the variable 'construction of knowledge' had a lower loading in both Asian studies. Remarkable is that the study orientation scales that have a negative loading in the Dutch study do not return in the Asian groups; however, in the Sri Lankan study a positive loading of the 'personally interested' scale is seen. The structure of the Indonesian meaning-directed factor showed more differences. They regard presence of loadings on the memorizing and rehearsing component and external regulation of the learning process, intake of knowledge and absence of loadings on personal interest.

Most noteworthy is the fact that in both Asian studies stepwise processing scales (Sri Lanka analyzing, Indonesia both analyzing and memorizing) load on the first factor. The same applies for external regulation scales (for Sri Lanka regulation of outcomes, for Indonesia regulation of process and outcomes). The two latter differences, combined with the differences in study orientation and conceptions of learning give this factor in the Asian context an unmistakably different flavour.

Also Factor 2 that in the Dutch study was marked by high loadings of stepwise processing strategies, external regulation and conceptions of learning as intake of knowledge receives a somewhat different content in the Asian studies. The loadings of the processing strategies are less high, while 'analysing' disappears from this factor in the Indonesian sample. Interestingly, all study orientations have moderate loadings on this factor.

Similarities and differences on Factors 3 and 4 can be best analysed in combination. In the Dutch study Factor 3 was described as an undirected strategy, defined by lack of regulation, an ambivalent orientation, and a wish for a stimulating, cooperative learning environment. Here Factor 4 was the application-oriented factor, with loading of a vocation and certificate orientation, a conception of learning that emphasized application of knowledge and concrete processing. Asian Factors 3 and 4 included loading of the same variables, but differently distributed over the two factors. Most remarkably factor 3 collects loadings of all learning conception scales; in case of the Indonesian study this is extended with moderate loadings of vocation and self-test orientations, while in the Sri Lankan study a personally interested orientation loads on this factor. The Asian Factor 4 is mostly characterized by high, but opposite loadings of 'lack of regulation' and an ambivalent orientation. Other loadings complement the undirectedness and passivity, making it a factor more similar to Factor 3 in the Dutch study.

Generally speaking rather similar patterns emerged when the Dutch and Sri Lankan studies are compared. The total amount of explained variance differs only $2.2 \%$ (52.2 vs $54.7 \%$ ). However, the amount of variance explained by the individual factors shows slightly different patterns; the Sri Lankan factors are more equal in weight than the Dutch and the Indonesian.

\section{Conclusions and discussion}

The general objective of the meta-analyses in this chapter was to identify the similarities and the differences in the reporting of learning strategies, learning conceptions, learning orientations and the internal structure of the learning patterns among Sri Lankan and other Asian students (Indonesian) and European (Dutch) students in terms of reported use and factor structure. The outcomes will be dealt with first by sub-question, after which two other perspectives will be taken on the results, i.e. learning environment and culture. 
Do Sri Lankan students differ from Dutch and Indonesian students in terms of the reported use of study strategies, orientations of learning and conceptions of learning?

This question was answered affirmatively. The differences were more between the Sri Lankan and Dutch group than the Sri Lankan and Indonesian group.

The differences between the three groups on reported use have been summarized in Table 7.5. A couple of striking differences can be observed: The number of significant differences between the Dutch and the Sri Lankan groups are greater (13) than between the two Asian groups (8). The Dutch students score higher on the processing strategies critical processing and on memorizing and rehearsing; on the eleven other subscales (one processing strategy, two out of three regulations strategies, four conceptions of learning and four learning orientations) the Dutch score lower, including the scales lack of regulation and ambivalent orientation. Differences between the two Asian groups did not regard regulation strategies, but mostly concerned study aims and learning conceptions. Indonesian students were more certificate, self-test and vocation oriented, they saw learning more as the intake and use of knowledge and less as stimulating education. Finally Indonesian students less often learned by relating and structuring and more often by memorizing and rehearsing.

Table 7.5. Summary of significant differences found in the comparisons of the inventory scale scores (the ILS or one of its versions) between the Sri Lankan study and a Western (Dutch, NL) and another Asian study (Indonesian. IN).

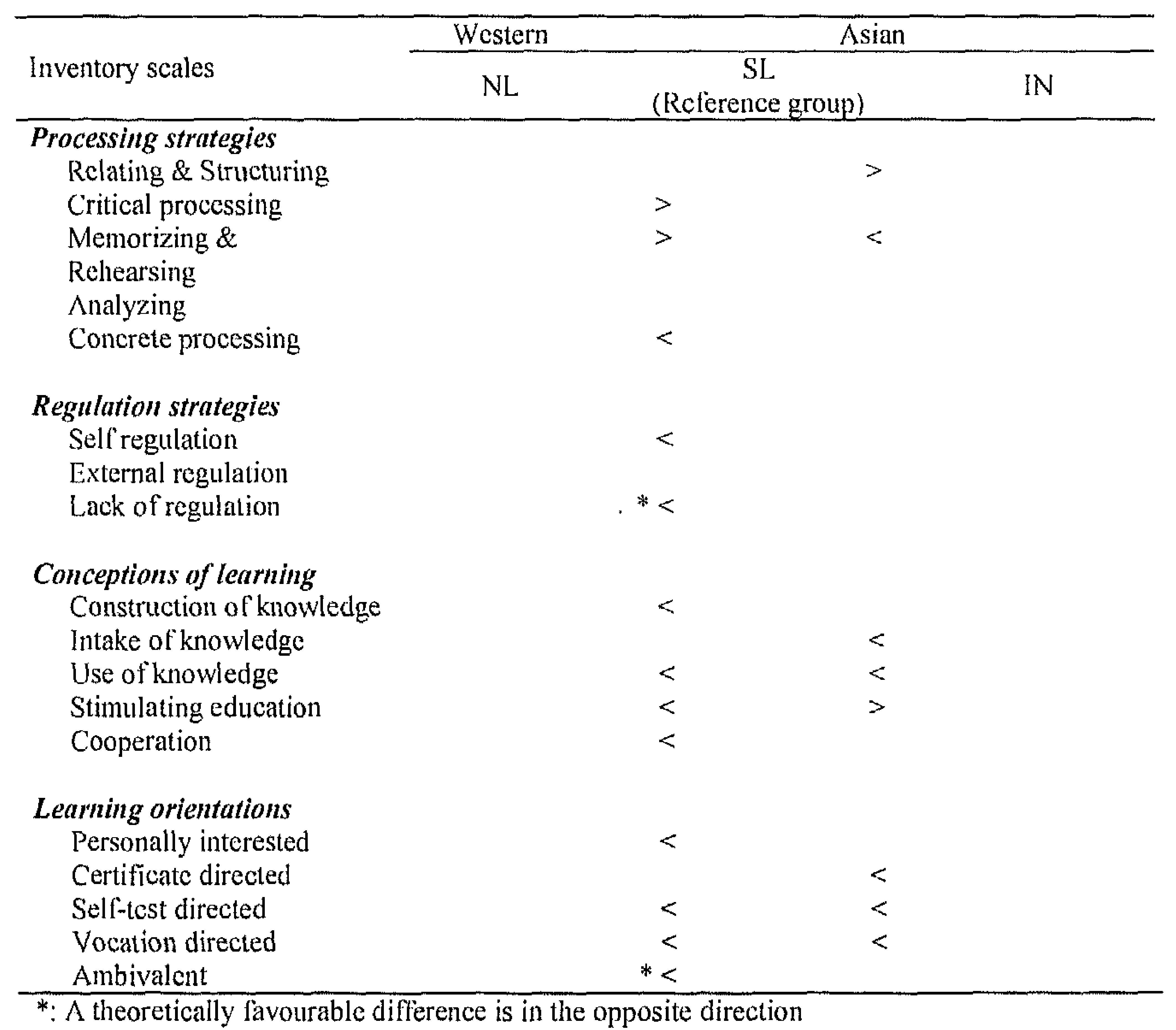


These outcomes are hard to interpret. The outcomes on three subscales especially raise questions about the outcomes. The high reported use of memorizing and rehearsing by the Dutch students contradicts findings related to the Asian paradox (Kember, 1996). On the other hand, this finding is corroborated by the interview results reported in Chapter 5 . The finding that Sri Lankan first year students score much less on memorising and rehearsing fits the results of Kember and Gow's (1991) study, which reported that compared to Australian students, Hong Kong students scored higher on deep approach scales and lower on surface approach scales. They challenged the anecdotal claim that Asian students are inherently more inclined to be rote learners. On the other hand, the Indonesian students also scored high on this scale, so maybe there is more variation in Asia than first expected. However, the findings on ambivalent study orientation and on perceived lack of regulation, in combination with a higher level of self-regulation in the Asian groups, cannot be theoretically explained, but does not fit the image of a self-regulated deep learner. This blurred picture can also be due to different national or cultural biases in answering the questionnaire and the different subscales. That is, that a cultural bias in the outcomes may have affected the overall (sub) means of the groups. Biases less likely affect factor structures. So combining these findings with the results on the other research questions might help to clarify this picture.

Similarities and differences in the internal structure observed in the two Asian and the Western cultures

Contrary to the reported use of learning strategies the factor structures of the three cultural groups showed large similarities. In all three cultural samples four factors were discerned. The internal structure of three of these factors shared some common features. They were the meaning-directed leaning pattern; the reproduction directed learning pattern and the undirected learning pattern.

Despite these similarities between the three cultures the two Asian factor solutions had a slightly different flavour. The Asian meaning-directed factor and reproduction factor had in addition to the known marking variables also loadings of a few other elements, i.e. stepwise processing and external regulation. The Asian reproduction-directed factor had loadings of other orientation scales in addition to the certificate orientation, suggesting that orientation is not a powerful factor regulating the learning behaviour among these students. The undirected learning style was characterized by high loadings of the scales lack of regulation and ambivalent orientation and negative loading of personal interest although the Indonesian loadings were the opposite.

Strikingly, one of the Asian factors was defined almost exclusively by conceptions of learning, i.e. idealistic learning pattern while a factor similar to the application-directed pattern was not discerned in the two Asian studies. In this respect, Lonka and LindblomYlänne (1996) compared the Finnish psychology students and medical students and reported, application orientation was more typical of medical students. Subsequently, Lindblom-Ylänne and Lonka (2000) showed that application directedness is an important dimension among advanced medical students. Similarly, Vermunt and Vermetten (2004) concluded that application-directed learning is a distinct learning dimension, which emerges late in its development. Thus, the absence of an application directed learning pattern among the Sri Lankan medical students could probably be due to the fact that these are first year students. Nevertheless, this may also reflect basic socio-cultural differences between Asians and the Europeans. On the other hand, different learning environments may be leading to the emergence of different kinds of learning patterns of this nature 
(Lonka \& Lindblom-Ylänne, 1996; Lindblom-Ylänne \& Lonka, 2000; Vermunt \& Minnaert, 2003) thus, learning environments may be yet another reason for the observed differences.

\section{Theoretically incongruent learning patterns among the three cultural groups}

Dissonance in learning patterns means that the expected coherent linkages between the learning components, i.e. learning conceptions, orientations and strategies, fail to appear in a recognizable form in terms of learning pattern as conceptualized (see Chapters 3 and 5). Not surprising the factor structure found in the Dutch sample was most congruent, all factors being defined by loadings of at least three of the learning components, which may be manifesting associations between the learning strategies students use and their learning conceptions and orientations (Vermunt, 1998).

In other words, learning activities employed by the Dutch students were guided or regulated by students' views on learning and their motives for studying. The Sri Lankan medical student sample demonstrated these associations or interrelations between learning elements in two of the four factors. In the present study such conceptual consonance was shown in the meaning directed dimension (factor 1) and reproduction dimension (factor 2). Although there is less coherence between the learning views, motives and actions than the learning styles identified in the Dutch study, it appeared to be certainly more than among the Indonesian students. Few recent studies have reported that among academically failing students the expected linkages between approaches to learning and perceptions of the learning environment seemed to disintegrate (Steirnborg \& Bandaranayake, 1996). However, this reason could not be applied to the Sri Lankan students, as the majority was successful at examinations. As pointed out by Ajisuksmo and Vermunt (1999) the other important difference confirmed by the present study is that the elements within a learning component generally showed high loadings on the same factor, while in the Dutch sample these loadings spread over different factors. The passive idealistic dimension is a good example of the above. This is another feature of dissonance, which is described as 'elements of learning styles found to be missing'. In summary, it seems that all five phenomena of dissonance discussed by Vermunt and Verloop (2000) have shown up among Sri Lankan, first year medical students; 1) lack of a distinct application-directed learning style; 2) lack of differentiation within learning components; 3) lack of integration between learning components; 4) incompatibility of learning strategies, conceptions and orientations and 5) missing learning elements within a factor.

The presence of such different patterns among first year students could on the one hand be explained as a cultural variation. On the other hand, it can also be viewed as a transitional phenomenon (Lindblom-Ylänne \& Lonka, 2000; Vermetten, Vermunt, \& Lodewijks, 1999a; Vermunt \& Verloop, 2000; Vermunt \& Minnaert, 2003; Vermunt \& Vermetten, 2004) in which the student finds a mismatch between the learning strategies the learning environment fosters and the learning strategies the student habitually uses. Given the strong teacher-centered school education system in Sri Lanka and the abrupt change in the medium of instruction in the university (Chapter 2), the first year in the university could be experienced as a very different learning context. This kind of "friction' between teaching and learning strategies at the onset of university life may be constructive in nature thus leading to the development of mature learning conceptions and learning practices or the other way round (Vermunt \& Verloop, 1999). However, further explanations are beyond the scope of the present investigation. 


\section{Learning environment as an explanatory factor}

Cultural differences between the Netherlands and Sri Lanka may lead to different patterns or styles of learning and use of learning activities. Kember and Gow (1990) made an interesting observation that documented goals of higher education are remarkably similar across different national systems of higher education regardless of the cultural setting. Typically these include the promotion of independent learning and critical thinking. One could also argue that these represent characteristics of the ideal student adjudged by the higher education system. Although it is often stated that the universities must help students to master higher levels of competencies like relating, structuring and critical judgment, in practice Sri Lankan teachers often seem to encourage a surface learning approach with over reliance on lectures as a method of instruction. While at examinations students are required to reproduce the information and knowledge transmitted in the classroom considerably, despite the fact that this practice is being criticized in many instances. On the other hand, this situation is deemed to improve as the students advance, as higher-level thinking is not emphasized in the first year where the thrust is on teaching the basic concepts of the discipline.

In the Asian context there appears a scarcity of staff trained in fundamentals of education. Although teacher-training programs are organized from time to time, some teachers do not consider it important to attend these or read recent literature on educational research. They hardly find time to meet and discuss with colleagues during faculty hours as the teachers are overburdened with teaching functions, service functions and peripheral faculty affairs, a trend that is seen over the last decade or so. Such situations could have negative consequences on the learning process.

\section{Asian culture as an explanatory factor}

"Guruvaraya" or teacher in the Sri Lankan society is a reliable, respected person. This term "guru" is derived from the Sanskrit word meaning "weighty" or "honorable". In India, Sri Lanka as well as Indonesia, this is what a teacher is called (Ajisuksmo, 1996; Hofstede, 2001). It is a custom to respect, listen to, often not to criticize or challenge one's teacher. Therefore, students will rather obey and follow their teachers. Students when asked to describe whom they would call an ideal student (as described in Chapter 5) three out of thirty one mentioned "a person who respects teachers", to quote one student, "Somebody who respects the teachers, a simple calm person, studies without grumbling. Always concerned about the teachers and try to make the teachers happy" this same student reported, "I think a student must respect his/her teachers... If they have doubts about their teachers then you cannot believe or learn from them ...It is important to develop a good impression about the teachers."

The Sri Lankan secondary education system, though had undergone many changes, is still a teacher centered and authoritarian system where the student is not supposed to argue or challenge the thinking process of the teacher. The observation that Asian cultures discourage the expression of thought is found to be valid even today. The impact of the family on shaping our beliefs and practices is extremely strong and mental programs set in childhood are quite difficult to change. Subsequently children develop their practices while in school. It is seen that the pair teacher-student is replacing the role pair parentchild, but basic values and behaviours are carried forward from one sphere into the other.

As Hofstede (2001) described: in countries where there is a large power distance situation such as is the case with Sri Lanka, the parent-child inequality is perpetuated by the teacher-student relationship. Teachers are treated with utmost respect, older teachers 
more than younger teachers. It is commonly seen that students stand up when a teacher enters a classroom. Teachers often outline the intellectual paths to be followed. In the classroom there is a strict order where the teacher initiates the communication, etc. A dominance of factual view of knowledge among teachers and learners can be seen as a domination of its cultural context. As opposed to the situation in low Power Distance (PDI) countries, i.e. the Netherlands, in high PDI countries the educational process is highly personalized. What is transferred is not seen as impersonal "truth" but as the personal wisdom of the teacher. This has led to authoritarian behaviour of teachers and veneration of teachers by the students. As a result, students even if they disagree with the teachers, avoid arguing with them. In such learning environments, opportunities to enhance critical thinking skills become limited.

Another cultural bias may have had differential effects on the questions where students were asked how much they agreed or disagreed with a statement. This was the case with the conceptions of learning and learning orientations items. The other scales required an answer in term of frequency. Maybe disagreeing with statements that come from an authority is more impolite in Asian than in Western cultures, which in turn may lead to different factor patterns.

It appears that the Asian thinking has many similarities, as the Indonesian society too shares a somewhat same line of thinking (Ajisuksmo, 1996). In the view of some Sri Lankan communities, grades (end result) are more important than the learning process. Hence teachers tend to focus more on transmitting information than on how the students process the information, what strategies they use in comprehending subject matter, how they regulate their learning process and understanding. Overloaded curricula, which are anyway the case in most medical schools and forms of assessments that require verbatim recall of facts invariably, encourage a surface learning approach. 


\section{Trends in the development of self-regulated learning among undergraduate medical students within the context of a traditional medical curriculum}

\section{Introduction}

As shown in chapter 3 , the development of expertise involves the process of developing domain specific knowledge and epistemological beliefs with a shift towards more selfregulated and meaning directed learning. It is also evident that the changes in the reported use of learning strategies among students are dependent on the learning context to a reasonable degree (Busato, Prins, Elshout, \& Hamaker, 1998; Christophoulus, Rohwer, \& Thomas, 1987; Eley, 1992; Gow \& Kember, 1993; Ramsden \& Entwistle, 1981; Trigwell, Prosser, \& Waterhouse, 1999; Vermetten, Lodewijks, \& Vermunt, 1999b; Volet, Renshaw, \& Tietzel, 1994). Moreover, students' perception of their learning environment is also related to the approach to learning that they adopt. According to Ramsden (1992), students who perceive the nature of assessment as encouraging memorization and recall and who perceive the workload demands of a subject as high are more likely to adopt a surface approach. Furthermore, it is also reported that the development of learning approaches within different kinds of medical curricula is significantly different and is very much influenced by the learning context provided by the educational institution (see chapter 3). These facts strengthen the argument of some researchers that a traditional curriculum seems to guide students towards the adoption of less desirable study approaches (Coles, 1985; Lindblom-Ylänne, 1999; Newble \& Clarke, 1986; Stiernborg \& Bandaranayake, 1996; Tan \& Thanaja, 1993). On the other hand, a study conducted in a medical school where a problem-based curriculum had been properly implemented, has shown that this medical school was able to guide students towards an elaborative, meaning directed learning pattern (Jayawickramarajah, 1993). However, empirical evidence on the developmental trends in learning strategies, conceptions and orientations among Sri Lankan medical students is non-existent and thus this was thought an area to be worth to be explored.

Furthermore, as described in chapter 3 , the issue of consistency and variability of learning strategies, conceptions and orientations among western university students has been addressed by several researchers. Vermunt and Vermetten (2004), in addressing the issue whether learning strategies are more like a consistent individual learning style or whether they are flexible and adaptive towards a specific situation, state that there is a person bound component and a context bound component in students' use of learning strategies. Thus empirical evidence suggests that the consistency versus variability of learning strategies does not yield an "or-or answer but an and-and answer" and that the learning strategies differ from each other in their degree of variability (Vermetten et al, 1999b: Vermunt \& Vermetten, 2004). It has been observed that the use of a memorizing processing strategy was relatively stable while a concrete processing strategy and lack of regulation showed a relatively high sensitivity to the course context. However, the degree of stability and variability in the reported use of learning strategies, conceptions and orientations within an Asian context has not been documented and thus the longitudinal study (study 3 ) described in this chapter is expected to further clarify the issue of consistency and variability among University students. 
Research on the interrelations of the learning patterns identified with the ILS reveals an interesting developmental phenomenon. Based on the results of a number of Dutch studies, Vermetten, Vermunt, and Lodewijks, (1999a) formulated a development hypothesis, which suggests that with the students' progress in education, the factor structure underlying their learning strategies, conceptions of learning and orientations will become more focused and reveal stronger interrelations. Subsequently, Vermunt and Vermetten (2004) conclude "that the development hypothesis holds true for students progressing within one type of education, while a contextual hypothesis explains the disintegration of the factor pattern when there is a change in the context such as a change from higher secondary to first year in the university". However, we do not know enough about the behaviour of Sri Lankan students and therefore, the studies reported in this empirical chapter are also directed at clarifying the issue of developmental theory underlying the ILS.

A study of the literature also revealed that longitudinal within subject studies to examine intra-individual differences in learning strategies within the context of a medical school in Sri Lanka are non-existent. Nevertheless, it is well known that students use different approaches to learning depending on the learning environment, curriculum, assessment and personal factors. Therefore, empirical evidence on development of learning strategies and learning patterns among undergraduate medical students of Sri Lanka was thought to be useful for educational decision-making. A question that is often posed in the Faculty Board is "Don't we achieve the same outcomes with the present system?" or "What is bad with the prevailing system which has produced us?" Hence, at a time when there is so much discussion on curricular reforms in the higher education system in Sri Lanka and medical education in particular, the present study was conducted to obtain useful information on the pattern of reported use of learning strategies and the developments in the learning conceptions and orientations of medical students, and their perceptions of the learning environment. Finally, it is also expected to produce reliable information for curriculum developers on the degree to which the educational context is successful in producing deep level academic learning, as the students' progress in academic life, and on the degree of consistency and variability of the different learning elements across time.

\section{Aims and objectives of the present study}

Against this background, the aim of the present study was to explore the pattern of development of learning strategies, orientations and conceptions, the consistency and variability of these learning aspects within the MBBS course of studies, and student perceptions of their learning environment in a traditional medical school. Due to practical reasons, a cross sectional study and two longitudinal studies were designed in order to study the developments in learning strategies, conceptions and orientations in the $2^{\text {nd }}$ MBBS course and then in the $3^{\text {rd }}$ and $4^{\text {th }}$ years of the undergraduate medical course. The research problem investigated in the current chapter was: "what is the trend in the development of learning strategies, orientations and conceptions among Peradeniya medical students and can the student perceptions of the learning environment be used as an explanatory factor for observed trends in learning patterns?"

\section{Research questions}

In summary, the research questions of the present study were:

1. Do learning strategies, conceptions of learning and learning orientations differ between three groups of students who differ in the study phase they are in, i.e. new entrants, $3^{\text {rd }}$ year and $4^{\text {th }}$ year medical students? 
2. Do reported learning strategies, conceptions of learning and learning orientations differ within two groups of students at two moments in time with an interval of 30 weeks and 50 weeks?

3. How does the factor structure underlying the ILS change during two stages of the MBBS course?

4. How consistent or variable are students' learning strategies, conceptions of learning and learning orientations?

5. How do first and fourth year students perceive their learning environments?

\section{Method}

The study design was realized within the 2 nd MBBS course and the $3^{\text {rd }}$ MBBS Part I and part II courses at the Faculty of Medicine, University of Peradeniya, Sri Lanka during May - June 2000, and July - August 2001. The first part of the study had a cross sectional design while the second part of this study had a within subject longitudinal design. Therefore some of the participants of study 3 also participated in study 4 .

\section{Study 3: Cross-sectional study}

The student characteristics, materials, and procedure of this study are described in Chapter 6.

Data analysis

Analysis of variance was used to measure variations in learning strategies among the three batches of students in the cross sectional study. In case of significant differences, a Bonferroni post hoc test was performed.

\section{Study 4: Longitudinal study}

\section{Participants}

These were the medical students of the 1999/2000 batch and the 1996/1997 batch who completed the inventory on both occasions time 1 and time 2 as shown in Table 8-1. The group comprised 144 of the 159 new entrant medical students (82\% of the total) and 92 of the 143 third year medical students (61\% of the total) of the University of Peradeniya, Sri Lanka. The response rates were more than $60 \%$, relatively higher compared to those reported in other longitudinal studies (Busato et al., 1998; Vermetten et al., 1999a).

Questionnaires were also distributed among the final year students. They were instructed to return the completed questionnaires to the Medical Education unit. Despite several reminders only 10 out of the 120 students who received a questionnaire responded. It could be that final year medical students had so much work to cover within that year that they could not spare 45 minutes of their time for this research.

\section{Materials}

Learning strategies, conceptions and orientations were measured in this study by means of the ARPM2 (both Sinhala and English versions of the inventory were used as a few students were unable to read and understand the Sinhala version). 


\section{Procedure}

The ARPM 2 was administered prior to the commencement of the $2^{\text {nd }}$ MBBS course (study 3, see Chapter 6) and towards the end of the $3^{\text {rd }}$ term of the $2^{\text {nd }}$ MBBS course of 1999/2000 batch of students (study 4). For the 1996/1997 batch, the same inventory was administered during the $1^{\text {st }}$ term of $3^{\text {rd }}$ MBBS part I course (study 3, see Chapter 6 ) and then towards the end of the $3^{\text {rd }}$ term in the $3^{\text {rd }}$ MBBS part II course (study 4). The rest of the procedure is the same as described in chapter 6 . Only those who completed both inventories at time 1 and time 2 as shown in Table 8.1, were included in the longitudinal study. All paired analyses were performed on these two groups; namely 144 first year students and 92 fourth year students.

Table 8.1. The times of administration of the ARPM in the longitudinal study

\begin{tabular}{lll}
\hline Batch & Time 1 & Time 2 \\
\hline $1999 / 2000$ & $\begin{array}{l}\text { Prior to the commencement of the After following 30 weeks of academic work } \\
\text { course } \\
\text { 1st term of } 3^{\text {rd }} \text { year }\end{array}$ & After following 50 weeks of academic work \\
\hline $1996 / 1997$ &
\end{tabular}

\section{Data analysis}

Paired samples t-tests were used to analyze the changes between the $1^{\text {st }}$ administration and the $2^{\text {nd }}$ administration, concerning all variables for the two batches of students separately. Principal component analyses with varimax rotation were performed on the ARPM scales after each administration (time 1 and time 2) for the $1^{\text {st }}$ year and the $4^{\text {th }}$ year batches, respectively. Pearson correlation coefficients were calculated between learning strategies, conceptions and orientations at individual student level for these two groups of students.

\section{Study 5}

This study investigated how the first and fourth year medical students perceive their learning environment.

\section{Students}

Subjects were 170 students ( $94 \%$ of the total) of the $1999 / 2000$ batch (first year) after completion of 40 weeks of academic work, and 122 (74\% of the total) fourth year students of the 1996/1997 batch after completing up to the 3rd MBBS part II examination.

\section{Materials}

To gain more insight in possible differences across the different stages of the MBBS course, additional variables were measured. This involved the assessment of student perceptions of their immediate course experiences. The instrument used to measure the students' perception of the learning environment was an adapted version of the Course Experience Questionnaire (CEQ), with 25 items, developed by Paul Ramsden and his associates (1998) in Australia. The CEQ was based upon the Course Perceptions questionnaire developed by Entwistle and Ramsden in Britain (Entwistle \& Ramsden, 1983) and has been used widely across universities in Australia to assess the quality of teaching (Trigwell \& Prosser, 1991). The items of the CEQ covered areas such as facilitation of learning, the assessment strategies and development of generic skills. The first year stu- 
dents completed the Course Experience Questionnaire, which also contained the Sinhalese translation of each item, along with its original item, while the $4^{\text {th }}$ year students completed the original CEQ that was in the English language.

The 24 items of the Course Experience Questionnaire (Ramsden, 1998) were scored on Likert's scale ranging from (1) "entirely disagree" to (5) "entirely agree". Based on the results of factor analysis, CEQ items are grouped into 5 scales as depicted in Table 8.2 (Ramsden, 1998).

Table 8.2 Description of the CEQ scales, and examples of items

\begin{tabular}{|c|c|}
\hline CEQ scale & Scale description and example of items \\
\hline Good Teaching & $\begin{array}{l}\text { Whether the teachers were successful in facilitating the learning proc- } \\
\text { ess. } \\
\text { "The teaching staff normally gave me helpful feedback on how I was } \\
\text { going." }\end{array}$ \\
\hline Clear goals and standards & $\begin{array}{l}\text { Whether the goals and objectives of the course were clear to the stu- } \\
\text { dent, } \\
\text { "It was often hard to discover what was expected of me in this course." }\end{array}$ \\
\hline Appropriate assessment & $\begin{array}{l}\text { Whether the examinations were testing memorization abilitics. } \\
\text { "Too many staff asked me questions just aboul facts." }\end{array}$ \\
\hline Appropriate workload & $\begin{array}{l}\text { Whether the amount of work to be completed within a specified time } \\
\text { was satisfactory. } \\
\text { "I was generally given enough time to understand things I had to } \\
\text { learn." }\end{array}$ \\
\hline Generic skills & $\begin{array}{l}\text { Whether the course was helpful in developing generic skills among the } \\
\text { learners. } \\
\text { "As a result of my course I feel confident about tackling unfamiliar } \\
\text { problems." }\end{array}$ \\
\hline
\end{tabular}

\section{Procedure}

The CEQ was administered only once to a particular batch of students and was filled in separately during curriculum time. In order to assess the perceptions of the first year students (1999/2000 batch), it was administered in the fourth term of the $2^{\text {nd }}$ MBBS course, to all the students, (new entrants who are now first year students), present on that day soon after a tutorial class, just as in the previous instances. Similarly, the original CEQ was administered to the third year batch of students who took part in the longitudinal study, at the end of the $3^{\text {rd }}$ term of their 3 MBBS part II course. Student participation was anonymous and voluntary. The students were instructed to reflect on the part of the MBBS course that they have completed for the last one-year in answering the questionnaire.

\section{Data analyses}

Reliability coefficients were computed for the five scales of the CEQ. For all items of the $C E Q$, item means and the minimum and maximum scores, and the frequency distribution of scores were also computed for the first years and the fourth years. 


\section{Results}

\section{Study 3}

Table 8.3 depicts results of one way analyses of variance concerning learning strategies, learning orientations and conceptions of learning, the mean scores obtained for the eighteen different learning variables of the new entrant, third year and fourth year undergraduate medical students. On three of the five cognitive processing variables, critical processing, memorising and rehearsing, and analyzing, the more advanced students were found to have significantly lower scores than new entrants. Scores of two of the regulation strategies i.e. self-regulation and external regulation were significantly lower among advanced students, while lack of regulation scores were significantly higher among the advanced students. Among the learning orientations, personal interest score was found to be significantly lower and ambivalence score was significantly higher with progress in education. Similarly, significantly high scores were observed in two of the conceptions of learning scales, use of knowledge and stimulating education among the advanced students.

Table 8.3. Means and standard deviations on ARPM-scales of three groups of students differing in study phase, F-values and significance levels of the differences between the means based on analysis of variance

\begin{tabular}{|c|c|c|c|c|}
\hline ARPM scale & $\begin{array}{c}\text { New entrants } \\
N=159 \\
\text { Mean (SD) } \\
\end{array}$ & $\begin{array}{c}\text { Third year students } \\
N=143 \\
\text { Mean (SD) } \\
\end{array}$ & $\begin{array}{c}\text { Fourth year students } \\
N=136 \\
\text { Mean (SD) }\end{array}$ & $F(2,419)$ \\
\hline \multicolumn{5}{|l|}{ Processing strategies } \\
\hline Relating \& Structuring & $3.34(0.76)$ & $3.28(0.80)$ & $3.19(0.87)$ & 1.52 \\
\hline Critical processing & $2.83(0.93)$ & $2.53(0.90)$ & $2.62(0.99)$ & $4.02^{*}$ \\
\hline Memorizing \& Rehearsing & $2.83(0.88)$ & $2.63(0.84)$ & $2.25(0.79)$ & $17.19^{* * *}$ \\
\hline Analyzing & $3.48(0.62)$ & $3.17(0.76)$ & $2.95(0.76)$ & $20.10^{* * *}$ \\
\hline Concrete processing & $3.28(0.79)$ & $3.41(0.92)$ & $3.34(0.88)$ & .85 \\
\hline \multicolumn{5}{|l|}{ Regulation strategies } \\
\hline Self regulation & $3.24(0.70)$ & $3.02(0.75)$ & $2.76(0.74)$ & $15.22 * * *$ \\
\hline External regulation & $3.20(0.59)$ & $3.23(0.61)$ & $2.68(0.62)$ & $35.54 * * *$ \\
\hline Lack of regulation & $2.50(0.78)$ & $2.93(0.74)$ & $3.02(0.80)$ & $19.11 * * *$ \\
\hline \multicolumn{5}{|l|}{ Conceptions of learning } \\
\hline Construction of knowledge & $3.91(0.59)$ & $3.99(0.47)$ & $3.95(0.50)$ & .90 \\
\hline Intake of knowledge & $3.64(0.54)$ & $3.67(0.44)$ & $3.63(0.53)$ & .24 \\
\hline Use of knowledge & $4.09(0.55)$ & $4.23(0.49)$ & $4.30(0.41)$ & $7.10^{* *}$ \\
\hline Stimulating education & $3.51(0.79)$ & $3.98(0.74)$ & $3.93(0.65)$ & $18.48^{* * * *}$ \\
\hline Co-operation & $3.72(0.74)$ & $3.64(0.77)$ & $3.74(0.79)$ & .57 \\
\hline \multicolumn{5}{|l|}{ Learning orientations } \\
\hline Personally interested & $3.67(0.65)$ & $3.52(0.68)$ & $3.31(0.61)$ & $10.51^{* * *}$ \\
\hline Certificate oriented & $3.39(0.74)$ & $3.26(0.75)$ & $3.34(0.73)$ & 1.10 \\
\hline Self-test oriented & $3.40(0.66)$ & $3.45(0.74)$ & $3.32(0.75)$ & 1.04 \\
\hline Vocation oriented & $4.08(0.74)$ & $4.12(0.61)$ & $4.11(0.62)$ & .12 \\
\hline Ambivalent & $2.54(0.78)$ & $2.66(0.71)$ & $2.99(0.76)$ & $13.46 * * *$ \\
\hline
\end{tabular}


Post hoc multiple comparisons of means were used to test differences among scale scores in the different stages of the MBBS course. One way analysis of variance followed by Bonferroni post hoc multiple comparisons indicated that new entrants had significantly higher scores for critical processing than the third year students. The scores did not differ significantly between the new entrants and the fourth years or between third years and fourth years. Reported use of memorizing and rehearsing strategy was significantly lower in the fourth years when compared to the third years and new entrants.

The analyzing strategy showed a similar trend of lower scale scores among advanced students compared to new entrants. Similarly, self-regulation scores were significantly lower in the third and fourth years compared to the new entrants. The use of external regulation strategies was significantly lower in the fourth year students compared to the third years and new entrants respectively. However, the lack of regulation score was significantly higher in the third years and fourth years than among the novices. It was also evident that the personal interested orientation scores were significantly lower in the advanced students in comparison with first years, while the reporting of an ambivalent orientation was observed to be significantly higher among fourth years, but not between the new entrants and the third year students. A positive feature was that third and fourth year students attached greater value to the conception of learning 'use of knowledge' than new entrants. In addition, stimulating education scale scores were also found to be significantly higher in the third year and fourth year groups than in the group of new entrants.

\section{Study 4}

Changes in the learning strategies, learning orientations, and conceptions of learning among first year students and third MBBS students

Results of the paired samples $t$-test for the first year batch of students $(\mathrm{N}=144)$ are presented in Table 8.4. The top part of the table shows that students have reported the use of four of the five processing strategies significantly less at time 2 . Concrete processing remained unchanged. Two regulation strategies that show a significant change are selfregulation, which has decreased, and lack of regulation, which has increased.

Two learning orientation scales, which showed significant changes in the course of time, were the personal interest scale, showing a decreased score and the ambivalence scale with an increased score during a period of three academic terms in the first year. The only conception of learning that has changed significantly is the scale 'stimulating education' and the students have endorsed that significantly more. 
Table 8.4. Paired samples $t$-tests for learning strategies, learning orientations. and conceptions of learning for tirst year students $(\mathrm{N}=144)$.

\begin{tabular}{|c|c|c|c|}
\hline $\begin{array}{l}\text { ARPM scale } \\
\mathrm{N}=144\end{array}$ & $\begin{array}{l}\text { At entry (T1) } \\
\text { Mean (SD) }\end{array}$ & $\begin{array}{l}\text { Term } 3(\mathrm{~T} 2) \\
\text { Mean (SD) }\end{array}$ & $t(d f=143)$ \\
\hline \multicolumn{4}{|l|}{ Processing strategies } \\
\hline Relating \& Structuring & $3.35(0.76)$ & $3.17(0.77)$ & $2.00^{*}$ \\
\hline Critical processing & $2.83(0.95)$ & $2.32(0.87)$ & $4.74 * * *$ \\
\hline Memorising \& rehearsing & $2.85(0.87)$ & $2.41(0.73)$ & $4.64 * * *$ \\
\hline Analysing & $3.48(0.59)$ & $3.02(0.68)$ & $5.91^{* * *}$ \\
\hline Concrete processing & $3.29(0.79)$ & $3.19(0.87)$ & 1.01 \\
\hline \multicolumn{4}{|l|}{ Regulation strategies } \\
\hline Self-regulation & $3.24(0.70)$ & $2.75(0.67)$ & $5.56 * * *$ \\
\hline External regulation & $3.20(0.56)$ & $3.11(0.60)$ & 1.33 \\
\hline Lack of regulation & $2.51(0.79)$ & $2.84(0.79)$ & $-3.29 * *$ \\
\hline \multicolumn{4}{|l|}{ Conceptions of learning } \\
\hline Construction of knowledge & $3.92(0.60)$ & $3.93(0.50)$ & -.20 \\
\hline Intake of knowledge & $3.64(0.55)$ & $3.74(0.45)$ & -1.50 \\
\hline Use of knowledge & $4.11(0.56)$ & $4.16(0.47)$ & -1.15 \\
\hline Stimulating education & $3.53(0.78)$ & $3.80(0.77)$ & $-2.89 * *$ \\
\hline Cooperative learning & $3.73(0.74)$ & $3.85(0.91)$ & -1.01 \\
\hline \multicolumn{4}{|l|}{ Learning orientation } \\
\hline Personally interested & $3.67(0.64)$ & $3.44(0.63)$ & $3.01 * *$ \\
\hline Certificate oriented & $3.39(0.74)$ & $3.39(1.18)$ & .18 \\
\hline Self-test oriented & $3.41(0.64)$ & $3.41(0.72)$ & .03 \\
\hline Vocation oriented & $4.08(0.74)$ & $4.10(0.79)$ & -.17 \\
\hline Ambivalent & $2.54(0.78)$ & $2.84(0.77)$ & $-2.85^{* *}$ \\
\hline
\end{tabular}

\section{Third year MBBS students}

Results of the paired samples $t$-test are presented in Table 8.5. Only two out of five processing strategy scales changed significantly (analyzing and memorizing) between the first term of third year and third term of fourth year of the MBBS course. These students reported that they use the memorizing and rehearsing strategy and the analyzing strategy (both of which stand for step-by step processing), significantly less after one academic year (time 2). However, along with it the reported use of relating and structuring or concrete processing strategies has not changed in the course of time. Within the regulation domain all three components have changed significantly. Self-regulation, external regulation and lack of regulation are reported significantly less at time 2 .

Although the students' reporting that they use stepwise processing strategies (memorizing and rehearsing, and analyzing) less frequently at time 2 could be viewed as a positive development, overall changes demonstrated in the different domains do not seem to be demonstrating a trend towards the development of a meaning directed learning style among the fourth year students. Table 8.5 also indicates a significant decrease in self-test directed orientation and vocational orientation as well as personally interested orientation. Four of the five conceptions of learning, namely construction of knowledge, intake of knowledge, use of knowledge and stimulating education, also decreased. 
Table 8.5. Paired samples $t$-tests for learning stratcgies. learning orientations. and conceptions of learning for third year MBBS students ( $N=92$ ).

\begin{tabular}{|c|c|c|c|}
\hline ARPM scales & $\begin{array}{c}\text { Third year lerm } 1 \\
\text { Mean (SD) }\end{array}$ & $\begin{array}{c}\text { Fourth year term } 3 \\
\text { Mean (SD) }\end{array}$ & $t(d f=91)$ \\
\hline \multicolumn{4}{|l|}{ Processing strategies } \\
\hline Relating \& Structuring & $3.19(0.91)$ & $3.05(0.76)$ & 1.41 \\
\hline Critical processing & $2.44(0.96)$ & $2.46(0.96)$ & -.14 \\
\hline Memorizing \& rehearsing & $2.58(0.87)$ & $2.24(0.75)$ & $3.95 * * *$ \\
\hline Analyzing & $3.03(0.59\}$ & $2.79(0.71)$ & $2.67 * *$ \\
\hline Concretc processing & $3.32(1.01)$ & $3.17(0.86)$ & 1.40 \\
\hline \multicolumn{4}{|l|}{ Regulation strategies } \\
\hline Self-regulation & $3.00(0.78)$ & $2.83(0.71)$ & $2.07 *$ \\
\hline External regulation & $3.21(0.66)$ & $2.73(0.57)$ & $7.07 * * *$ \\
\hline Lack of regulation & $3.07(0.75)$ & $2.74(0.67)$ & $4.47 * * *$ \\
\hline \multicolumn{4}{|l|}{ Conceptions of learning } \\
\hline Construction of knowledge & $4.0(0.48)$ & $3.53(0.68)$ & $6.35 * * *$ \\
\hline Intake of knowledge & $3.74(0.44)$ & $3.55(0.62)$ & $2.67 * *$ \\
\hline Use of knowledge & $4.30(0.46)$ & $4.10(0.68)$ & $3.01 * *$ \\
\hline Stimulating education & $4.11(0.75)$ & $3.77(0.71)$ & $3.06 * *$ \\
\hline Co-operation & $3.76(0.74)$ & $3.69(1.10)$ & .67 \\
\hline \multicolumn{4}{|l|}{ Learning orientations } \\
\hline Personally interested & $3.48(0.74)$ & $3.30(0.67)$ & $2.26 *$ \\
\hline Certificate oriented & $3.34(0.76)$ & $3.25(0.82)$ & .92 \\
\hline Self-test oriented & $3.56(0.75)$ & $3.20(0.79)$ & $3,40 * *$ \\
\hline Vocation oriented & $4.16(0.5)$ & $3.87(0.77)$ & $2.97 * *$ \\
\hline Ambivalent & $2.78(0.75)$ & $2.81(0.84)$ & -.35 \\
\hline
\end{tabular}

Comparison of the factor structures of the first year batch of siudents at time 1 and time 2

Table 8.6 reveals the factor analysis results for the first year group of students $(N=144,82$ $\%$ of the total batch) at time 1 (first administration) and at time 2 (second administration).

Factor 1 in both instances, show high loadings of relating \& structuring, critical processing, analyzing, concrete processing and self-regulation strategies, personally interested orientation and construction of knowledge, all of which are the defining characteristics of a meaning directed learning pattern. However, at time 2, in addition to those, a moderate loading of external regulation of results shows up.

Table 8.6 also reveals that factor 2 , the reproduction directed pattern, and factor 3 , the passive idealistic type, are printed in just the reverse order at time 2 in comparison with the freshmen. The passive idealistic factor at time 1 had, in addition to high loadings of all learning conceptions, relatively low loadings of vocation directed and self-test directed learning orientations and of memorizing \& rehearsing. At the second time the loadings of two learning conceptions were decreased.

The differences in the reproduction-oriented factor noted in the second administration are that the loadings on memorizing and intake of knowledge scales have increased and in addition to the two loadings on orientation scales, certificate directed and vocation directed, a loading shown up for the scale self-test directed. Thus, the factor structure does not appear to have become stronger and more coherent among the different learning aspects after the first three terms of study in the faculty. 
Chapter 8

Table 8.6. Factor loadings of ARPM-scales in a 4-factor Varimax solution for medical students at the commencement of the Medical studies ( $\mathrm{T} 1^{*}$ ) and during the third term of the $2^{\text {nd }}$ MBBS course (T2) (principal component analyses: $N=144$; loadings between -.25 and .25 omitted).

\begin{tabular}{|c|c|c|c|c|c|c|c|c|}
\hline \multirow{2}{*}{ ARPM Scale } & \multicolumn{2}{|c|}{ Factor 1} & \multicolumn{2}{|c|}{ Factor 2} & \multicolumn{2}{|c|}{ Factor 3} & \multicolumn{2}{|c|}{ Factor 4} \\
\hline & $\mathrm{T} 1^{*}$ & 12 & TI & $\mathrm{T} 2$ & TI & $\mathrm{T} 2$ & T1 & $\mathrm{T} 2$ \\
\hline \multicolumn{9}{|l|}{ Processing strategies } \\
\hline \multicolumn{9}{|l|}{ Deep } \\
\hline RELA & .84 & .85 & & & & & & \\
\hline CRIT & .80 & .78 & & & & & & \\
\hline \multicolumn{9}{|l|}{ Stepwise } \\
\hline MEMO & & & .29 & .58 & .48 & & & \\
\hline ANAL & .62 & .76 & & .28 & & & & \\
\hline Concrete & .68 & .69 & & & & & & \\
\hline \multicolumn{9}{|l|}{ Regulation strategies } \\
\hline \multicolumn{9}{|l|}{ Self } \\
\hline LPO & .69 & .77 & & & & & & \\
\hline $\mathrm{LC}$ & .63 & .50 & & & & & -.28 & -.41 \\
\hline \multicolumn{9}{|l|}{ External } \\
\hline LP & & & & .69 & .70 & & & \\
\hline LO & & .41 & & .54 & .69 & & & \\
\hline Lack & & & & & .25 & & .72 & .68 \\
\hline \multicolumn{9}{|c|}{ Conceptions of learning } \\
\hline CONS & .42 & .36 & .66 & & & .75 & & \\
\hline INTA & & -.29 & .75 & .56 & .34 & .44 & & \\
\hline USE & .37 & & .72 & & & .75 & & \\
\hline STIM & & & .73 & & & .68 & & \\
\hline COOP & & & .72 & & & .53 & & \\
\hline \multicolumn{9}{|l|}{ Learning orientations } \\
\hline PERS & .48 & .41 & & & & .25 & -.56 & -.59 \\
\hline CERT & & & & .59 & .52 & & .38 & .37 \\
\hline SETE & & & .38 & .52 & & & & .34 \\
\hline VOCA & & & .35 & .56 & .58 & & & \\
\hline AMBI & -.26 & & & & & & .79 & .76 \\
\hline Eigen value & 3.79 & 3.98 & 3.08 & 2.53 & 2.16 & 2.31 & 1.99 & 2.11 \\
\hline$\%$ explained variance & 18.97 & 19.94 & 15.40 & 12.68 & 10.82 & 11.55 & 9.96 & 10.54 \\
\hline Cumulative \% & 18.97 & 19.94 & 34.38 & 32.62 & 45.20 & 44.18 & 55.16 & 54.71 \\
\hline
\end{tabular}

Deep - Deep processing, RELA - Relating \& structuring, CRIT - Critically processing, MEMO - Memorizing and rchearsing, ANAL- Analyzing, Concrete - Concrete processing, Self - Self regulation, LPO Learning process \& outcomes, LC - Learning contents, External - External regulation, LP - Learning process, LO - Learning outcomes, Lack - Lack of regulation, CONS - Construction of knowledge, INTA - Intake of knowledge. USE - use of knowledge, STIM -Stimulating education, COOP - Cooperation, PERS - Personally interested, CERT - Certificate oriented, SETE - Self test oriented, VOCA - Vocation oriented, and ABMI - Ambivalent

Comparison of the factor structures of the 1996/1997 batch of students at time 1 and time 2

In Table 8.7, the factor structures of the third MBBS (1996/1997) batch of students $(\mathrm{N}=92,61 \%$ of the total batch) at time 1 and time 2 are compared. As depicted in this Table, factor 1 still represents the meaning directed learning pattern. This factor 1 , characterized by loadings on relating and structuring, critical processing, analyzing, concrete processing, self regulation of learning, personally interested learning orientation and a 
construction of knowledge learning conception, seems to show lack of integration between domains at time 2 (in the $4^{\text {th }}$ year). That is, factor 1 discerned at time 2 , is not defined by a loading of the conception of learning, "construction of knowledge" as expected and instead a low loading on the conception, use of knowledge is present. A moderate loading of the scale external regulation of results is also apparent. This points to a breakdown of factor structure.

Table 8.7. Factor loadings of ARPM-scales in a 4-factor Varimax solution for medical students during the first term of $3^{\text {rd }}$ MBBS course $\left(3^{\text {rd }}\right.$ year) and during the third term of the $3^{\text {rd }}$ MBBS part 11 course $\left(4^{\text {th }}\right.$ year) (principal component analyses; $N=92$; loadings between -.25 and .25 omitted).

\begin{tabular}{|c|c|c|c|c|c|c|c|c|}
\hline \multirow{2}{*}{ ARPM Scale } & \multicolumn{2}{|c|}{ Factor 1} & \multicolumn{2}{|c|}{ Factor 2} & \multicolumn{2}{|c|}{ Factor 3} & \multicolumn{2}{|c|}{ Factor 4} \\
\hline & $3^{\text {rd }}$ year & $4^{\text {th }}$ year & $3^{\text {rd }}$ year & $4^{\text {th }}$ year & $3^{\text {rd }}$ year & $4^{\text {th }}$ year & $3^{\text {rd }}$ year & $4^{1 / 1}$ year \\
\hline \multicolumn{9}{|l|}{ Processing strategies } \\
\hline \multicolumn{9}{|l|}{ Deep } \\
\hline RELA & .82 & .82 & & .28 & & & & \\
\hline CRIT & .78 & .73 & & & -.25 & & & -.24 \\
\hline \multicolumn{9}{|l|}{ Stepwise } \\
\hline MEMO & & & .62 & & & .78 & & \\
\hline ANAL & .68 & .69 & .26 & & -.35 & .38 & & \\
\hline Concrete & .70 & .78 & & .27 & -.29 & & & \\
\hline \multicolumn{9}{|l|}{ Regullation strategies } \\
\hline \multicolumn{9}{|l|}{ Self } \\
\hline LPO & .82 & .82 & .33 & & & .23 & & \\
\hline $\mathrm{LC}$ & .44 & .50 & .37 & & -.41 & .52 & & \\
\hline \multicolumn{9}{|l|}{ External } \\
\hline LP & & & .78 & & & .77 & & \\
\hline LO & & .31 & .79 & & & .62 & & \\
\hline Lack & & & & & .76 & & & .64 \\
\hline \multicolumn{9}{|c|}{ Conceptions of leaming } \\
\hline CONS & .67 & & & .68 & & & .29 & -.35 \\
\hline INTA & & & .60 & .71 & .33 & & .43 & .47 \\
\hline USE & .64 & .26 & & .78 & .28 & & .34 & \\
\hline STIM & .30 & & & .85 & & & .77 & \\
\hline COOP & & & & .63 & & & .53 & \\
\hline \multicolumn{9}{|l|}{ Learning orientations } \\
\hline PERS & .38 & .36 & & .54 & -.54 & & .40 & \\
\hline CERT & & & .45 & & & & .41 & .79 \\
\hline SETE & .34 & & .49 & .49 & .29 & .47 & .27 & \\
\hline VOCA & & .35 & .43 & .53 & & .38 & .61 & \\
\hline AMBI & & -.34 & & & .82 & & & .70 \\
\hline Eigen value & 4.50 & 3.80 & 3.05 & 3.73 & 2.44 & 2.65 & 2.12 & 2.13 \\
\hline$\%$ explained variance & 22.52 & 19.00 & 15.27 & 18.66 & 12.22 & 13.25 & 10.64 & 10.65 \\
\hline Cumulative $\%$ & 22.52 & 19.00 & 37.78 & 37.65 & 50.00 & 50.90 & 60.64 & 61.55 \\
\hline
\end{tabular}

Deep - Dcep processing, RELA - Relating \& structuring, CRIT - Critically processing, MEMO - Memorizing and rchearsing, ANAL- Analyzing, Concrete - Concrete processing, Self - Self regulation, LPO Learning process \& outcomes, LC - Learning contents, External - External regulation, LP - Leaming process, LO - Learning outcomes, Lack - Lack of regulation, CONS - Construction of knowledge, INTA - Intake of knowledge, USE - use of knowledge, STIM -Stimulating education, COOP - Cooperation, PERS - Personally interested, CERT - Certificate oriented. SETE - Self test oriented, VOCA - Vocation oriented, and $\mathrm{ABMI}$ - Ambivalent 
The reproduction directed learning pattern (factor 2), characterized by loadings of stepwise processing strategies, external regulation of the learning process, a certificate directed learning orientation and the learning conception intake of knowledge, shows some features of dissonance in the fourth year. That is, at the second time a loading of the learning conception intake of knowledge and a loading of the certificate directed learning orientation, are missing. This same factor is not defined by a conception of learning in the fourth year as was seen in the third year. Hence, the reproduction directed learning factor known to be characterized by loadings of stepwise processing strategies, external regulation of learning, a certificate directed learning orientation and the learning conception intake of knowledge, shows some features of dissonance, i.e. the phenomenon of missing elements of learning styles (described in chapter 3 ) in the fourth year.

In the third year, the undirected factor has high loadings of ambivalent orientation and lack of regulation scales and negative loadings of scales such as concrete processing and critical processing, self-regulation and personal interest. However, with the same students this factor is defined by high loadings of an ambivalent orientation, a certificate directed learning orientation (in addition) and lack of regulation at time 2 . Thus, the undirected learning factor also shows a breakdown of factor structure in the fourth year.

The passive-idealistic factor too showed lack of integration between learning domains and lack of differentiation in learning conceptions from year 3 to 4 . In general, when the students progress academically, it is evident that a breakdown of factor structure occurs and even the relations between the defining learning components become weaker. Moreover a clear, distinct application directed learning pattern was not apparent among the advanced students. Almost all the characteristics of dissonance (Vermunt \& Verloop, 2000) described in chapter 3 of this thesis, were revealed when the factor structures of the two administrations are compared.

Intercorrelations of ARPM scale scores between time 1 and time 2 for the two batches of medical students

Table 8.8 presents the Pearson correlation coefficients of the different ARPM scale scores in the two administrations. In order to measure the degree of stability of the different domains of learning, the correlations between the third year and the fourth year were calculated.

Table 8.8. Inter-correlations between time 1 and time 2, for the first year batch $(N=144)$ and the fourth year batch $(\mathrm{N}=92$ ), for learning strategies, learning orientations, and conceptions of learning.

\begin{tabular}{|c|c|c|c|c|c|c|c|c|c|c|}
\hline \multicolumn{11}{|c|}{ Learning Strategies } \\
\hline$N$ & RELA & CRIT & MEMO & ANAL & $\mathrm{CONC}$ & SELF & EXTE & LACK & & \\
\hline 144 & .03 & .02 & .02 & .07 & .02 & .12 & .09 & .08 & & \\
\hline 92 & $.34 * *$ & $.43 * *$ & $49 * *$ & $.33 * *$ & $.42 * *$ & $.45^{* *}$ & $.45 * *$ & $.49^{* *}$ & & \\
\hline \multicolumn{11}{|c|}{ Personological domain: Learning Orientations and Conceptions of Learning } \\
\hline $\bar{N}$ & CERT & VOCA & SETE & PERS & $\mathrm{AMBI}$ & INTA & CONS & USE & STIM & COOP \\
\hline 144 & $.17^{*}$ & .03 & .05 & .05 & .03 & .02 & .07 & .09 & .09 & .13 \\
\hline 92 & $.38^{* *}$ & .09 & .08 & $.43^{* *}$ & $.40^{* *}$ & $.23^{* *}$ & $.29^{* *}$ & 0.12 & 0.14 & $.31^{* *}$ \\
\hline \multicolumn{11}{|c|}{$\begin{array}{l}\text { Note* } p<.05, * * p<.01 \text {. } \\
\text { RELA = relating and structuring; CRIT = critically processing; } \mathrm{MEMO}=\text { Memorizing; ANAL = Analyz- } \\
\text { ing; CONC = Concretizing; SELF = self regulation; EXTE = External regulation; LACK=lack of regula- } \\
\text { tion; CERT = certificate oriented; VOCA = vocationally oriented; SETE = self-test oriented; PERS = per- } \\
\text { sonally interested; AMBI= Ambivalent; INTA = Intake of knowledge; CONS= construction of knowledge; } \\
\text { USE= Use of knowledge; STIM = Stimulating education; COOP = Co-operative learning. }\end{array}$} \\
\hline
\end{tabular}


For learning strategies $r$ varied between 0.33 and 0.49 . Learning orientations showed coefficients between 0.38 and 0.40 while two orientations, namely, vocation oriented and self-test directed, did not show a significant relationship between the pattern of scoring by individual students at time 1 and time 2 .

For three of the five learning conceptions, coefficients were between 0.23 and 0.31 while a significant correlation between the scores of the two administrations was not shown for the other two conception scales, use of knowledge and stimulating education. In the case of first year students $(n=144)$ the learning strategies, conceptions of learning and learning orientations, other than the learning orientation certificate oriented, $r=0.17$ did not show a significant correlation. The results obtained for the first year students, that the intercorrelations were lower than 0.17 for 17 out of 18 scales, is unique.

\section{Study 5}

\section{Internal consistencies of the Course Experience Questionnaire (CEQ) scales}

Ramsden (1991) reported the range of the internal consistencies (Cronbach alpha values) for the different scales as between .71 and .87 . The reliabilities of the two scales in the present study i.e. good teaching and generic skills, of the CEQ were acceptable and comparable to previous studies (see Table 8-9), while the internal consistencies of the other three scales were unacceptably low for the first year students and the fourth year students. Therefore, the results are expressed at item level for the first year students and fourth year students, respectively.

Table 8.9. Internal consistencics (Cronbach alpha, $\alpha$ ) of the five CEQ scales for the two batches of medical students, first year students (CEQ items along with the Sinhalese translations, $N=170$ ) and fourth year stidents $(N=122)$, CEQ original version.

\begin{tabular}{lcc}
\hline \multicolumn{1}{c}{ CEQ scales } & \multicolumn{2}{c}{$\alpha$} \\
\cline { 2 - 3 } & First year & Fourth year \\
\hline Good teaching & .78 & .78 \\
Generic skills & .72 & .78 \\
Appropriate assessment & .11 & .02 \\
Appropriate workload & .32 & .38 \\
Clear goals \& standards & .14 & .20 \\
\hline
\end{tabular}

Table 8.10 depicts the perceptions of the learning environment of 1 st year ( $94 \%$ of the total) and $4^{\text {th }}$ year (74\% of the total) medical students. Interestingly, most of the students in both batches agreed with the items "The work load was too heavy" and "There was a lot of pressure on me to do well in this course" (mean values are highest). As can be seen in Table 8.10 the items on which students from both batches scored lowest were: "I was generally given enough time to understand the things I had to learn'; 'It was always easy to know the standard of work expected'; 'I usually had a clear idea of where I was going and what was expected of me in this course'; 'The staff put a lot of time into commenting on my work'; and 'The staff made a real effort to understand difficulties I might be having with my work." 
Chapter 8

Table 8.10. Means and standard deviations of CEQ-items of first year and fourth ycar students, and t-tests of the differences between the mcans

\begin{tabular}{|c|c|c|c|}
\hline Item & $\begin{array}{l}1^{\text {s1 }} \text { year mean } \\
(\mathrm{SD})\end{array}$ & $\begin{array}{c}4^{\text {th }} \text { year } \\
\text { mean }(S D)\end{array}$ & l-test \\
\hline \multicolumn{4}{|l|}{ Good Teaching } \\
\hline The teaching staff of this course motivated me to do my work best. & $2.96(1.02)$ & $3.14(1.12)$ & NS \\
\hline The staff put a lot of time into commenting on my work. & $2.23(1.02)$ & $2.16(1.04)$ & NS \\
\hline $\begin{array}{l}\text { The staff made a real effort to understand difficulties I might be having } \\
\text { with my work. }\end{array}$ & $2.49(1.10)$ & $2.69(1.14)$ & NS \\
\hline $\begin{array}{l}\text { The teaching staff normally gave me helpful feedback on how I was going. } \\
\text { My lecturers were extremely good at explaining things } \\
\text { The teaching staff worked hard to make their subjects interesting }\end{array}$ & $\begin{array}{l}2.84(0.99) \\
2.95(1.01) \\
2.90(1.07)\end{array}$ & $\begin{array}{l}2.94(1.21) \\
2.68(1.17) \\
2.92(1.22)\end{array}$ & $\begin{array}{l}\text { NS } \\
* \\
\text { NS }\end{array}$ \\
\hline \multicolumn{4}{|l|}{ Clear Goals and Standards } \\
\hline \multirow{4}{*}{$\begin{array}{l}\text { It was always easy to know the standard of work expected } \\
\text { I usually had a clear idea of where I was going and what was expected of } \\
\text { me in this course. } \\
\text { It was often hard to discover what was expected of me in this course } \\
\text { The staff made it clear right from the start what they expected from stu- } \\
\text { dents }\end{array}$} & $2.43(1.02)$ & $2.55(1.09)$ & NS \\
\hline & $2.65(1.00)$ & $2.68(1.15)$ & NS \\
\hline & $3.40(1.19)$ & $3.33(1.19)$ & NS \\
\hline & $2.76(1.11)$ & $2.76(1.18)$ & NS \\
\hline \multicolumn{4}{|l|}{ Appropriate Assessment } \\
\hline \multirow{3}{*}{$\begin{array}{l}\text { To do well in this course all you really necded was a good memory } \\
\text { The staff seemed more interested in testing what I had memorized than } \\
\text { what I had understood } \\
\text { Too many staff asked me questions just about facts }\end{array}$} & $2.98(1.33)$ & $3.85(1.18)$ & $* * *$ \\
\hline & $3.38(1.25)$ & $3.25(1.11)$ & NS \\
\hline & $3.19(1.04)$ & $2.83(1.02)$ & $* *$ \\
\hline \multicolumn{4}{|l|}{ Appropriate Workload } \\
\hline \multirow{4}{*}{$\begin{array}{l}\text { The workload was too heavy. } \\
\text { I was generally given enough time to understand the things I had to learn } \\
\text { There was a lot of pressure on me to do well in this course } \\
\text { The sheer volume of work to be got through in this course meant it } \\
\text { couldn't all be thoroughly comprehended }\end{array}$} & $4.79(0.70)$ & $4.53(0.87)$ & $* *$ \\
\hline & $1.89(1.06)$ & $1.89(1.09)$ & NS \\
\hline & $3.94(1.16)$ & $3.83(1.23)$ & NS \\
\hline & $4.14(1.00)$ & $3.24(0.90)$ & $* * * *$ \\
\hline \multicolumn{4}{|l|}{ Generic Skills } \\
\hline The course developed my problem-solving skills & $3.55(0.94)$ & $2.88(1.03)$ & $* * *$ \\
\hline The course sharpened my analytic skills. & $3.62(0.86)$ & $2.73(1.06)$ & $* * *$ \\
\hline \multirow{2}{*}{$\begin{array}{l}\text { The course helped me to develop my ability to work as a team member. } \\
\text { As a result of my course, I feel confident about tackling unfamiliar prob- } \\
\text { lems. }\end{array}$} & $3.46(1.13)$ & $3.12(1.17)$ & * \\
\hline & $3.52(0.87)$ & $3.01(1.04)$ & $* * *$ \\
\hline \multirow{2}{*}{$\begin{array}{l}\text { The course improved my skills in written communication. } \\
\text { My course helped me to develop the ability to plan my own work. }\end{array}$} & $3.65(0.85)$ & $3.20(1.10)$ & $* * *$ \\
\hline & $3.51(0.95)$ & $2.87(1.08)$ & $* * *$ \\
\hline \multicolumn{4}{|l|}{ Ungrouped item } \\
\hline $\begin{array}{l}\text { The assessment methods employed in this course required an in-clepth un- } \\
\text { derstanding of the course content. }\end{array}$ & $3.56(1.18)$ & $3.26(1.21)$ & * \\
\hline
\end{tabular}

Table 8.10 also shows that for 11 of the 24 items, first year and fourth year students scored significantly different (indicated by stars). Compared to the fourth year students, first year students scored higher on all the generic skills items, i.e. 'The course: developed my problem solving and analytic skills, developed the ability to work as a team member, improved my skill in written communication and that as a result of the course I feel confident about tackling unfamiliar problems', and 'Too many staff asked questions just 
about facts'. However, contrary to what was expected, fourth year students reported a significantly higher score for the item 'to do well in the course all really needed was a good memory' while they scored significantly less on 'the assessment methods employed in this course required an in-depth understanding of the course content'.

As depicted in Table 8.11 , a majority of the first year students (100 students; 61\%) indicated that the overall satisfaction of the course was $60 \%$ or more.

Table 8.11. Responses obtained to the last item in the CEQ "The overall satisfaction of the course as a percentage"

\begin{tabular}{lcc}
\hline Rank & $\begin{array}{c}\text { First year students } \\
(\% ; N=170)\end{array}$ & $\begin{array}{c}\text { Fourth year students } \\
(\%: N=122)\end{array}$ \\
\hline 0 & 1.2 & 0 \\
20 & 9.1 & 5.7 \\
40 & 29.1 & 30.3 \\
60 & 52.7 & 30.3 \\
80 & 6.7 & 9.8 \\
100 & 1.2 & 0 \\
Not ranked & - & 23.7 \\
\hline
\end{tabular}

In the case of fourth-year student group, 37 students (30\%) indicated that the degree of overall satisfaction with the course was $40 \%$ while another 37 students (30\%) indicated that the overall satisfaction with the course was $60 \%$. However, $24 \%$ of the fourth year students did not respond to this final question. It could be that students were not satisfied with their teaching learning experiences, but that they did not want to express their views fearing possible unpleasant consequences.

\section{Conclusions and discussion}

The purpose of this investigation was to explore the puzzle of student learning within the context of a conventional medical curriculum. Therefore, in this chapter a unidimensional as well as a multi dimensional perspective was taken in studying the developments in academic learning. In addition, contextual as well as personological factors have been considered in trying to explain the results of the empirical studies. Furthermore analyses were conducted to examine the variability and stability of different learning domains during the course of time.

Do learning strategies, conceptions of learning and learning orientations differ between three groups of students who differ in the study phase they are in, i.e. new entrants, third year and fourth year medical students?

This first research question was answered affirmatively. Results of study 1 show marked differences between the responses of different groups of students in both, cross sectional as well as longitudinal studies. Our attempts at exploration of course, come with certain limitations that must be acknowledged. Scores between the new entrants, third year and fourth year medical students were found to be significantly different for three of the five processing strategies memorizing and rehearsing, analyzing, and critical processing. Although the finding that the scores of two strategies indicative of a reproduction directed style were significantly lower among advanced students, is viewed as a positive development, a correspondingly high scores for strategies indicative of a meaning directed style 
being absent was somewhat confusing. In fact, critical processing scores were much lower in the latter part of the course. Therefore, when all the changes are considered jointly, contrary to what was expected, the present study revealed the development of a less desirable learning style across time.

With respect to the regulation strategies, contrary to what was expected a significant reduction in the use of self- regulation was observed and a significant increase in the reporting of lack of regulation was noticed. The reported use of external regulation has decreased. The overall trend in the reported use of regulation strategies as revealed by the study is not satisfactory. It was also revealed that personally interested orientation scale scores were significantly lower while ambivalence scores were higher among the advanced students, these were exactly the opposite trend of what had been expected. Among the conceptions of learning, stimulating education as well as use of knowledge has endorsed positively and the scores increased over the years. The fact that advanced students seem to endorse the idea of learning as use of knowledge is a favourable trend. However, in general, the results of the cross sectional study pointed towards the development of an undirected learning style among Sri Lankan medical students. The changes observed were incoherent and were not consistent with the studies of Newble and Clarke (1986) and Clarke (1986). Nevertheless, this is not the only study, which failed to reveal an increase in meaning oriented learning. Findings of cross sectional studies reported by Busato et al. (1998), Stiernborg and Bandaranayake (1996), and Lonka and Lindblom-Ylanne (1996) also showed similar results. Based on the changes in learning elements that were observed, it is reasonable to state, as far as the cross sectional study among Sri Lankan medical students is concerned, that it points towards the development of an undirected learning pattern with academic progress. Thus, the findings of the present study were different from the results of cross sectional studies of Lonka and Lindblom-Ylänne (1996) and Busato et al. (1998), in which a clear systematic relationship between the year of study and the learning style was not evident.

Do reported learning strategies, conceptions of learning and learning orientations differ within two groups of students at two moments in time with an interval of 30 weeks and 50 weeks?

Within student developments in the first year of the MBBS course

First, within student developments in different domains of learning among first year students during the first three terms of $2^{\text {nd }}$ MBBS course were examined. During the first year of the course it was revealed that students reported the use of two strategies indicative of a meaning directed learning pattern (relating and structuring, critical processing), as well as two strategies indicative of a reproduction oriented learning pattern (memorizing and rehearsing and analyzing), significantly less after a 30 week period of academic learning. Among the regulation strategies they reported the use of self-regulation significantly less while increased scores for lack of regulation were evident. Personally interested orientation decreased and ambivalence increased while stimulating education has been endorsed significantly less. The new entrant scores were reflective of the students' study orientation, brought by them to the medical training. However, it was seen that processing scale scores representative of a reproduction directed learning were reduced during the first three terms in medical school. Along with it the self-regulation score was not found to be increasing while the reported personal interest was decreasing during the 
first three terms in medical school. Vermetten et al. (1999a) were able to confirm the independence between reproduction directed and meaning directed learning. This study also confirms the independence of the two styles. However, medical students as a group did not show improved reported quality of learning during the course of studies as was shown by Vermetten et al. (1999a) between a period of two semesters. Finally, based on the above findings, it can be concluded that the students after 3 terms of work show a systematic increase of scores in all aspects of an undirected learning style: lack of regulation, ambivalence and stimulating education.

\section{Within student developments in the middle part of the MBBS course}

The changes observed after a period of 50 weeks ( 5 terms) of course work do not show a development towards a meaning directed style or an application directed style, which are considered desirable by all medical schools. It was disturbing to notice decreased scores on personal interest and construction of knowledge. Unlike with the first years, a systematic increase in the style construct (strengthening of the association between aspects of learning) was not obvious in this group. The results obtained were somewhat inconsistent with previous research conducted in other disciplines such as Law, Psychology and Sociology using the $I L S$ as the test instrument (Busato et al. 1998; Vermetten et al. 1999a; Vermunt \& Minnaert, 2003) in that those studies reported an increase in meaning directed learning scores.

The present study based on the strategy scales that changed significantly, along with the direction of change (a significant decrease in personal interest, increase in ambivalence, lack of regulation and stimulating education), points towards development of learning strategies (particularly in the third and fourth years) in an undesirable direction from the point of view of tertiary education. It seems like the educational environment as opposed to stimulating a meaning directed or application directed learning style is leading towards an undirected style. Contrary to the finding of Scott, Markert, and Dunn (1998) that the critical thinking skills improve modestly among third year medical students, with academic progress, the present study failed to reveal an increase in the use of critical processing strategies. However, in interpreting the results one needs to keep in mind the limitations of self-report instruments. Therefore, with a certain degree of caution though, it is not unfair to conclude that the prevailing learning environment in Faculty of Medicine of the University of Peradeniya, Sri Lanka, may be discouraging reproduction directed learning to some extent but at the same time is not successful in encouraging meaning oriented learning. Our findings are consistent with an Australian study conducted by Stiernborg and Bandaranayake (1996), where they showed a reduction in meaning directed scores from first year-to-year six. Hence, the present results confirm previous research and add to the mounting evidence that conventional curricula foster approaches to learning that are less desirable.

Students as a group reported the use of a memorizing and rehearsing strategy, an aspect of a reproduction directed style, significantly less at time 2 . Since we were able to demonstrate a change in memorizing and rehearsing and analyzing strategies, which represents reproduction directed learning, one wonders whether they are as stable as were thought to be. Although Vermetten et al. (1999a) have also confirmed the idea of Schmeck (1988) that the reproduction directed learning has been crystallized, by the time they enter higher education, it is still arguable. In addition, Newble and Gordon (1985) using the Lancaster Approaches to Study Inventory have also concluded that the scores on reproducing orientation did not differ significantly in a cross sectional design while 
within the same study a sequential rise in the scores for meaning orientation was noted among medical students.

\section{How does the factor structure underlying the ILS change during two stages of the MBBS course?}

The third research question refers to multidimensional development in student learning and is concerned with changes in relations between different domains of learning while the student progress in their studies. It should be noted that both factor structures in the two longitudinal studies were compared, but no formal statistical technique has been applied. As was expected during the first three terms in the medical school the factor structure showed less coherent relations between the learning strategies, conceptions of learning and learning orientations. Vermetten et al. (1999a) reported a breakdown in factor structure in the first year (first semester) of university education among Dutch students as well. Since it is a period of acclimatization from the point of students, where the students have to get accustomed to the medium of instruction, i.e. English and university type of education, it was hypothesized that the factor structure is not strengthened and sometimes may fall apart. The fact that the results were not in favour of a substantial breakdown in factor structure within the first year is to be explained by the context hypothesis described at the beginning. One wonders whether the context is more or less similar to the school environment. In favour of the latter argument, first year in conventional curriculum is the period during which students learn the basic sciences, it could well be that educational environment is similar to science education they have had so far with hardly any exposure to clinical medicine and applications of knowledge. Therefore, if at all little cognitive disequilibrium is encountered and as a result, the factor structure has not strengthened and a distinct application directed factor has not emerged.

With respect to the changes observed during the third MBBS stage it was expected that according to the developmental hypothesis (Vermunt \& Vermetten, 2004) as well as the Model of Domain Learning (Alexander, Jetton, \& Kulikowich, 1995), the factor structures would strengthen during this period of the course. However, as shown in this study, the factor structures seemed to disintegrate as the student group advanced by five academic terms during the third MBBS course. Therefore, the context hypothesis is brought in to explain the changes in the factor structure during this period. For some groups of students the expected interrelations between learning conceptions, learning motives and learning processes did not show up, this phenomenon resembles the concept that Meyer (1991) named "dissonance" (described in Chapter 3). Vermetten et al. (1999a) in their study documented that the factor structure was less clear in the 1st year of studies than in the second year. Recently, Vermunt and Minnaert (2003) also documented a breakdown in factor structure among a group of Belgian Sociology students in the third trimester within a student oriented learning environment. However, when comparing the factor structure of Roosendaal and Vermunt (1996; as cited by Vermunt \& Vermetten, 2004) in the upper phase of secondary education, with those in the first year in university, the factor structure seemed to fall back to pieces. Both unclear factor patterns herald a period of new type of education or a new educational context. This may indicate a period of "friction", a period during which students find that ideas of knowledge and how to go about learning are no longer adequate (Vermunt \& Verloop, 2000).

Thus, the breakdown in factor structure, i.e. disintegration within learning strategies, learning conceptions and learning orientations among the advanced students can be explained as follows. Medical students following a traditional curriculum during the $3^{\text {rd }}$ 
MBBS period have to study a number of para-clinical subjects, attend clinical clerkships, and are subjected to many assessments. Moreover, during this period medical students are taught subjects, which might not be all that relevant in the immediate practice. Hence, some teachers wonder whether this arrangement has led to less desirable consequences. Overloaded curriculum, frequent examinations, clinical clerkships all may be adding on to the extra volume of work and thereby making learning extremely stressful. Recently Lindblom-Ylänne and Lonka (2000) reported that advanced medical students in a traditional curriculum, who had dissonant learning style profiles, also revealed that many of them went through a process of change in their ways of studying. The dissonant patterns in this study too may indicate a similar change among students. The results of the present study were inconsistent with some of the previous studies (Vermetten et al. 1999a; Vermunt \& Verloop, 2000) in that a consolidation of factor structures among the more advanced students or the development of an application directed factor was not evident.

\section{How consistent or variable are students' learning strategies, conceptions} of learning and learning orientations?

In two previous studies Vermetten et al. (1999a, 1999b) addressed the question of consistency in strategy use by comparing within-student variations across different university courses as well as over a period of time in the university. They reported the existence of both individual consistency and contextual variation. Although students reported changes in their strategic behaviour over time, they also showed individual consistency in the same period. However, the results of the present study did not reveal such strong intercorrelations as shown by Vermetten et al. (1999a) between the different learning dimensions during the course of studies. The results of this study can be explained as that, particularly during the first three terms, the individual student responses on different aspects of learning varied considerably. It suggests that changes in the learning environment and increased experience in university education affected individual students in different ways, so that the rank order of scores in the two instances has been changed considerably among first year students. This was in contrast to what was reported by Vermetten et al. (1999a).

The novice students seem to have changed their conceptions on learning, learning motives and processing and regulation strategies considerably within the first three terms.

This heralds a period of change among medical students. Thus one may argue, with careful intervention, the student approaches could be turned towards the desirable direction during this period. On the other hand, the expression of greater degree of variability among learning conceptions and learning orientations perhaps could be due to the nature of the items concerning these variables. The more general and abstract items in these scales may have caused difficulties in answering for the first time. However, the internal consistencies of these scales (particularly the conception scales) were high in all the studies.

The advanced medical students showed comparatively low levels of intercorrelations for the strategy scales and some of the conceptions and orientations. Thus, among advanced medical students, it can be speculated that changes in the learning environment and experience in education, with respect to learning strategies, orientations, and conceptions, affected at least most of the students in more or less the same way, without a change in the rank order of students on the different learning variables. Furthermore, results obtained for the fourth year students of the present study supports the view that learning strategies are more stable than conceptions of learning or learning orientations, 
therefore, were consistent with Vermetten et al. (1999a). At least among the advanced students, correlations of the strategy scales were higher than those of conceptions and orientations. However, according to the model proposed by Vermunt (1998) learning strategies should have been less stable than learning conceptions and learning orientations. Neither the results of the present study nor those of Vermetten et al. (1999a) were in support of this model.

\section{How do first and fourth year students perceive their learning environ- ments?}

Since some of the scales of the CEQ gave unacceptable reliabilities, item scores were considered in interpreting results. A majority of the students in both cohorts expressed that work load was heavy and that they were not given sufficient time to understand things they had to learn. In addition, the majority indicated that they had problems in figuring out what was expected of them at the end of the course and that at times when they found it difficult to understand the subject matter, the support offered by staff was not satisfactory. In explaining the contribution of perceptions of the learning environment on the development of learning patterns, it is not unreasonable to state that the perceived heavy workload as was endorsed by both groups of students is the dominant factor, which may promote a shift towards a less desirable learning style. Results of both studies revealed that reproduction directedness is decreasing, however, a corresponding increase in meaning orientation or vocation orientation was not found while application directed learning style was not shown. Vermunt and Vermetten (2004) pointed out that application directed learning develops as a separate dimension later in academic life while Lindblom-Ylanne and Lonka (2000) concluded that advanced medical students had higher scores for application directed learning. However, these results of the present study were inconsistent with those two previous studies.

Interestingly, there were also differences between the first and fourth year students in their course experiences. These differences were most prominent on the topics of 'generic skills', 'appropriate workload', and 'appropriate assessment'. Fourth year students, as opposed to the first years, scored much less on "workload is heavy". Compared to fourth year students, first years were not so much in agreement with the fact that all one needs to do well in their course of study is a good memory. Furthermore, the first years also endorsed that their course was helpful in development of generic skills while the fourth years scored much less on these aspects.

Changes observed with respect to conceptions of learning and learning orientations when taken as a whole are also inconsistent with a number of previous studies (Lonka \& Lindblom-Ylanne, 1996; Perry, 1970; Vermetten, et al. 1999b). Although the finding that advanced students less often endorsed the idea of learning as the intake of knowledge can be seen as a favourable trend, the fact they less often endorsed the idea of construction of knowledge and use of knowledge is contradictory. From the CEQ results it is obvious that the reported degree of satisfaction with the course shows a decreasing trend from first to the fourth years. Furthermore, the ARPM scale ambivalence showed a significant increase in its mean score among the advanced students. Student perception of an overloaded curriculum, preponderance of didactic teaching, discipline based teaching approach and objective assessments could be speculated as major contributors to these observed changes.

In general, all three investigations reported in this chapter revealed a trend of developing a less desirable learning pattern with a clear increase in lack of regulation and 
ambivalence scores, stimulating education and a decrease in personal interest among the advanced medical students. This supports the idea of Vermetten et al. (1999a) that changes in the conceptions of learning and learning orientations can partially explain changes in the learning strategies domain. 


\section{General conclusions and discussion}

\section{Introduction}

The central theme of this thesis is "the learning behaviour and its development of Sri Lankan medical students within the context of a traditional curriculum". Therefore, a deliberate attempt was made to explore the cognitive processing activities, regulation activities, conceptions of learning and learning orientations and their interrelations among several groups of undergraduate medical students. The objective of this final chapter is to discuss the results and conclusions of the empirical chapters at a general level and evaluate the entire project as a whole. At the beginning of this chapter the conceptual model underlying the project, main results and discussion points of the previous chapters are summarized, in order to show how the objectives described in chapter one has been realized.

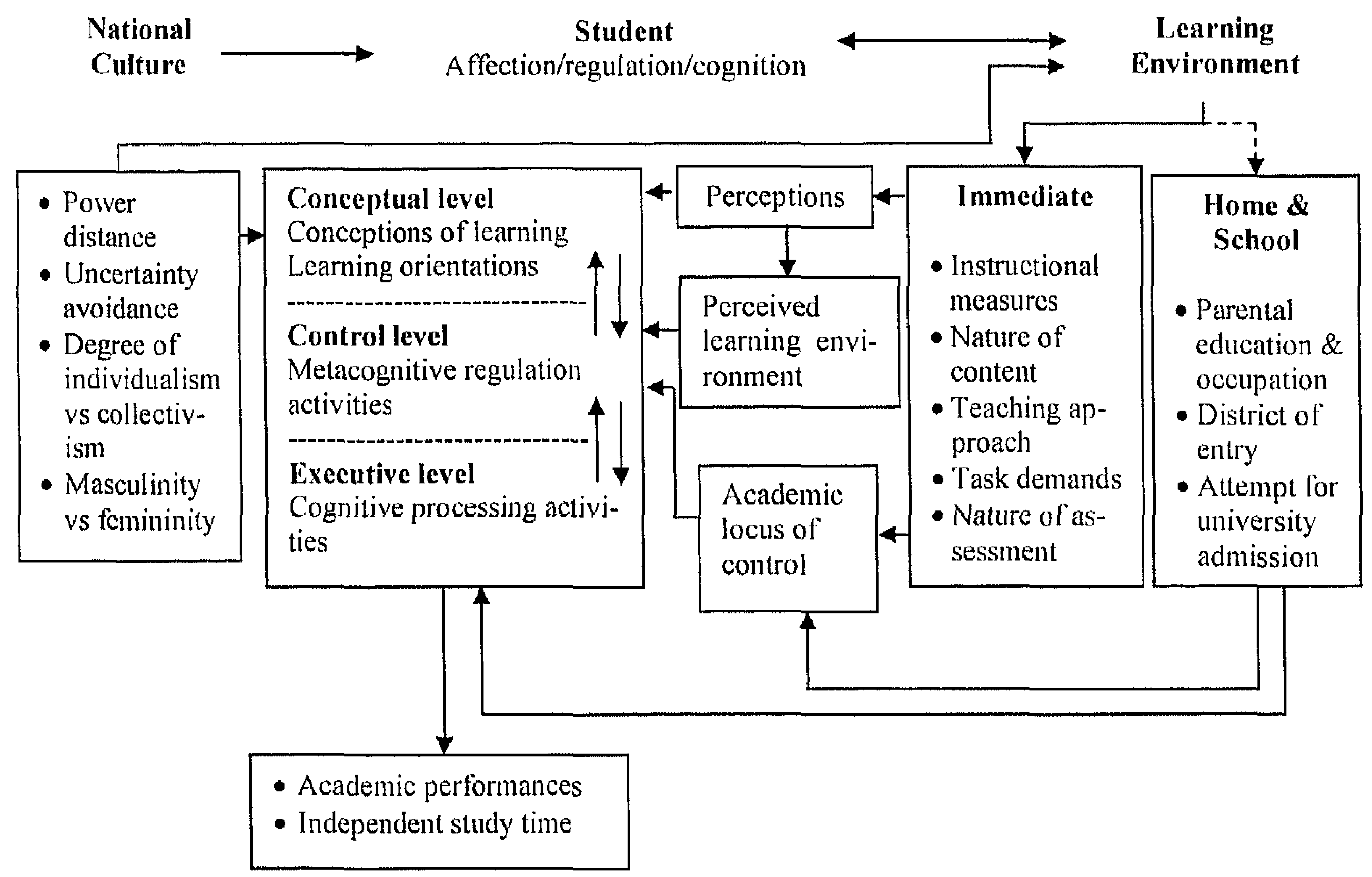

Figure 9.1. Student learning in higher education - a conceptual model underlying the dissertation.

As depicted in the conceptual model underlying the dissertation (fig. 9.1), the quality of student learning could be influenced by a variety of personal and contextual factors, including the national culture. The research project investigated the influences of the factors depicted in figure 9.1 on the quality of student learning. Towards this end, several research questions were formulated and investigated as described in chapters $5,6,7$ and 8 . 


\section{Main findings of the overall project: summary and discussion}

The main findings of the overall project are highlighted in this section. The overall objective of the project was to explore the learning patterns as well as the effects of contextual and personological factors on aspects of learning of Asian medical students within a conventional curriculum. The overall objective had been worked out to eight major objectives, which in turn were investigated in the empirical studies. Thus, the project, carried out at the faculty of Medicine University of Peradeniya, Sri Lanka had eight major objectives (see chapter 1 and 4 ).

1. To identify and describe the learning strategies, conceptions of learning, academic locus of control, learning motives, attributes of an "ideal student", and views on teaching and learning in higher education of Sri Lankan undergraduate medical students at the commencement of their studies.

2. To develop a valid and reliable diagnostic instrument, the Adyayana Rata Prakasha Malawa (ARPM) that could be used to get an overview of the study habits and learning styles or patterns of Sri Lankan undergraduate medical students in the Faculty of Medicine, University of Peradeniya.

3. To investigate relations between ARPM scale scores and academic performance, level of parental education and occupation, district from which the student had secured entry to medical school, average reported study time, gender, attempt in which university selection is secured, and dimensions of academic locus of control.

4. To explore the attribution pattern (academic locus of control) of Sri Lankan medical students at different stages of the MBBS course.

5. To identify the similarities and differences in the reporting of learning strategies, conceptions and orientations among first year university students within a cross-cultural perspective.

6. To identify consistency and variability of the use of learning strategies, learning orientations and conceptions of learning across time and contexts among medical students.

7. To explore the developmental trends in respect of the four learning components (the use of processing strategies, regulation strategies, reporting of conceptions of learning and learning orientations), among Sri Lankan medical students within the context of a traditional curriculum.

8. To identify possible contextual factors that may promote the observed changes in learning patterns.

The objectives were translated into following research questions, which in turn were answered in the empirical chapters.

1. What are Sri Lankan medical students' study strategies, conceptions of learning, attribution patterns, values, beliefs, expectations of university teaching and learning and study motives?

2. Can the ILS be adapted to form a reliable and valid diagnostic instrument?

3. What are the learning patterns of Sri Lankan medical students?

4. What are the associations between students' use of learning strategies, their learning conceptions and orientations on the one hand and contextual factors (such as parental education and occupation, and district from which the student secured university entry), personal factors (such as gender, attempt at which university admission was secured, independent study time), and academic performance on the other? 
5. Does the national culture play a major role in explaining the students' study strategies and the interrelations between the different aspects of learning?

6. What are the trends in the development of learning strategies?

7. What are the students' perceptions of the existing learning environment?

The findings with respect to the above questions are discussed in the light of existing theoretical knowledge under broad headings derived from the questions. It is expected that the outcomes of the empirical studies will form the baseline for future research on learning behaviours of undergraduate medical students in Sri Lanka.

\section{Sri Lankan medical students' ways of learning: the qualitative study}

The qualitative study described in chapter 5 indicated that during senior secondary education, as far as cognitive processing activities are concerned, analyzing in a stepwise manner and memorizing and rehearsing are the most commonly used strategies. The interviews further highlighted that Sri Lankan learners, similar to the Chinese and the Japanese learners, combine understanding and memorization, as opposed to their western counterparts (Kember, 1996; Marton, Watkins, \& Tang, 1997; Marton, Wen, \& Wong, 2005; Purdie, Hattie, \& Douglas, 1996). However, the students hardly described the use of critical processing strategies during this period. This could be due to the fact that critical processing was not encouraged by the secondary education system. A majority of the students indicated that their learning processes were regulated by the university entrance examination. Due to the competitive nature of this examination, students expressed that their learning process was, to a large extent, under external control. This can be viewed as a sensible option, particularly in an environment where private tuition is believed to be helpful in getting the required aggregate score.

Similar to the observation of Kember (1996), it is also evident that these Sri Lankan students seek understanding but at the same time explicitly try to commit pertinent information to memory. Given the nature of assessment in education, this combined approach (strategic approach) to study may be highly successful as far as examination performance is concerned (Bloomfield, Harris, \& Hughes, 2003). As was expected, none of the students admitted that they faced a situation of inability to regulate their learning process during the senior secondary education, which is explained partly by the fact that there exists strong teacher control during this period of education. In addition, the fact that these medical students, like those in the other parts of the world, are high achieving students (Lindblom-Ylänne, 1999; Rennie, 2000), would also explain the absence of the reporting of lack of regulation of learning among them at this point of time.

Furthermore, as described in chapter 5, the new entrant medical students reported a somewhat constructivist conception of learning as opposed to simple memorization of content, while a majority of the students also indicated in their descriptions that the learner is active (implicitly or explicitly) in the learning process. They used only a folk notion of representation. These results were consistent with the observations of Lonka, Joram and Bryson (1996) in that these researchers reported a positive relationship between level of general education and the expressions of learning as a constructive process, the learner as an active participant and the use of terminology suggestive of mental representations. The fact that medical students refrained from using terminology suggestive of mental representations is in line with the finding that such expressions were a characteristic of expert psychology students in the Lonka et al. (1996) study. 
Purdie et al. (1996) revealed that, contrary to the common opinion, Japanese students were far less likely than Australian students to view learning as memorizing and reproducing. However, when it came to description of their real learning activities, the Japanese indicated they used memorization and rehearsal significantly more than Australian students. Van Mil (2005) reported a similar observation with a group of Chinese students studying in a Dutch University. This mismatch between conceptions of learning and actual use of learning strategies was also observed during the analysis of the present interviews. It may well be that new entrant medical students in general reported a strategic approach characterized by a high degree of adaptation to the competitive learning environment, thus displaying incoherent linkages between the different learning elements. This also suggests the possibility that Sri Lankan students' learning activities are more under control of the learning environment.

The present study also revealed that a vast majority of the Sri Lankan students attribute academic failure to lack of effort, which is an internal and a controllable cause. This is viewed as a favourable trend (Ames, 1992) and adds strength to the view that the Asian learners attribute their performances mainly to effort and study skill (Drew \& Watkins, 1998; Elliot \& Bempechat, 2002; Hess \& Azuma, 1991; Purdie et al., 1996). The observed attribution style of Sri Lankan students enhance motivation and repeated effort in failure situation while attributing failure to lack of ability has a detrimental effect on continued motivation. From the discourse of students, it was evident that the students were aware of their capabilities and believe they have control over their own learning, therefore, were ready to exert greater effort in order to succeed in the subsequent attempt.

In the view of several researchers an internal locus of control discourages a surface approach to studying, which would have had a negative impact on academic achievement (Drew \& Watkins, 1998; Watkins \& Astilla, 1984). This finding also suggests the possibility that revelation of an internal, controllable locus of control is beneficial to the learner in terms of academic achievement (Woolfolk, 1998). The results of the present study as well as a number of studies investigating the attribution pattern of students (described in chapter 6) suggest that Asian culture promotes this kind of attribution behaviour (Drew \& Watkins, 1998). Therefore, it is possible that this phenomenon is to some extent responsible for the high achieving nature of Asians, which has led to the Asian stereotype "brainy Asian" (Biggs, 1991).

However, contrary to what was expected among Sri Lankan medical students, in the cross sectional design, it was also revealed that the percentage of students who attributed their academic failures to effort showed a marked reduction over time. Given the fact that in the cross sectional study advanced students reported significant low scores for the personal interest scale, this former trend could possibly be a reflection of the effect of the learning environment on the student. Certain effects of the learning environment, assessment strategies in particular, may give rise to a feeling of lack of personal control over the learning task. Since it is widely believed that the achievement nature of the Asian students is partly due to the cultural bias for attributing their success and failure to effort (Biggs, 1991; Drew \& Watkins, 1998), this decreasing trend of attributing failure to internal and controllable causes should be of concern to the faculty as that could have serious consequences on motivation and achievement.

The attributes of an "ideal student" revealed by this sample of Sri Lankan medical students are: (1) hard working person, (2) exerting a lot of effort, (3) not a "book worm", (4) somebody who is capable of applying knowledge (5) a person with a range of abilities (6) a person with good communication skills, and (7) a person who can successfully work 
in a team. Some of these findings are in line with the claim that Asian students place particularly high value on academic engagement (Biggs, 1991). These values will no doubt lead to effective learning. However, the fact that "respecting one's teachers" was also described as an attribute of an ideal student (although by a few students), demonstrates the degree of influence of Asian culture (described in chapter 7) on one's way of thinking and learning (Hofstede, 2001; Nahn, 2006). Similarly, Purdie et al. (1996) indicated that Japanese mothers value "compliance with authority", "obedience in good grace" and "cooperation with teachers". They were of the view that these values may to some extent be responsible for the demonstration of behaviours such as compliance, obedience and reluctance to question the teacher, among Asian learners as opposed to Australian counterparts. Furthermore, the students in this study expressed the view that, unlike in the schools, a university teacher should play the role of a guide and provide sound academic and emotional support while the responsibility for learning should lie with the student in higher education. These findings also support the claim that Asian students place particularly high value on academic engagement (Biggs, 1991).

\section{From the ILS to ARPM: reliability and validity}

The question "Can the ILS be adapted to measure learning patterns of Sri Lankan undergraduate students?" yielded an affirmative answer. In chapter 6 of this dissertation it is concluded that the adapted Sinhalese version of the Inventory of Learning Styles (ILS), Adyayana Rata Prakasha Malawa (ARPM), is a suitable instrument to study the four domains of learning (cognitive processing, regulation activities, conceptions of learning and learning orientations) among Sri Lankan university students. Reliabilities of most of its subscales, in terms of internal consistencies were comparable to the Dutch results (Vermunt, 1998; Vermetten, 1999). Very recently, Nahn (2006) also validated the ILS among a group of Vietnamese medical students.

In short, the present study along with the results of Ajisuksmo and Vermunt (1999), confirmed the validity of a western model to assess learning styles among Sri Lankan medical students.

\section{Learning patterns of Sri Lankan medical students}

The internal structure of three of the four factors discerned by the present study, meaning directed, reproduction directed and undirected learning, were similar to those reported in a number of studies carried out with European university students. Based on the findings of Lindblom-Ylänne and Lonka (2000), it was expected to find a distinct application directed learning pattern among the more advanced medical students. However, a factor similar to application directed learning was not identified in the Sri Lankan study. Absence of a clear, distinct application directed pattern was also reported in the Indonesian study carried out by Ajisuksmo and Vermunt (1999). Instead, similar to the observation of Ajisuksmo and Vermunt (1999), a passive undirected factor showed up with loadings almost exclusively of conceptions of learning, among all groups of medical students, irrespective of the year of study. Subsequently, Nahn (2006) also reported a similar passive factor among Vietnamese medical students. Thus, at one point of time it was queried whether the emergence of the passive undirected factor or the phenomenon of absence of some of the learning elements within a learning pattern is peculiar to Asians. However, 


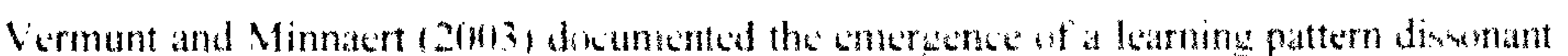

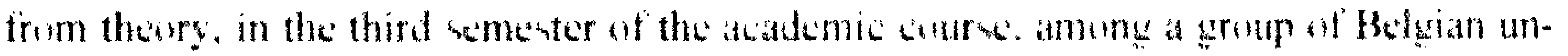

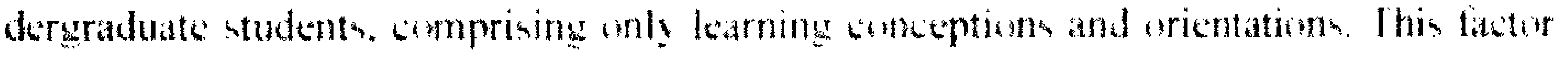
was named as the helied factur. They argued that this phenomem on could be a transent. developmental phenemenon during which the learner's existing strategies, orientations and conceptions are challenged to some estent. Thum the latter shernation suggeste that any further explanation in bey and the soupe of the present study and requires comparion of more vench studies carried out in different cultural contexts.

\section{Relations between learning patterns and personal and contextual fac- tors and academic performance}

In chapter 6 it is revcaled that the result ohtained are not consistent with these of previous studies carried wat in medical schools. which have investigated an association between learning approaches and academic perfirmance among medical students. 'The association between Approaches to Studying Inventory - ASI (Fntwistle \& Ramsden. 1983) scores and academic pertormance was measured in an Autralian medical school istiernbory \& Bandaranavake, 1996 ): they reported that meaning orientation and its subscales tended to be positively related to academic performance while reproducing orientation was negatively related to academic performance, although the correlations were small. These results were generally consistent with other studies among medical students using ASI (leiden, Crosby, \& Follmer. 1940: vewble \& (larke, 1986). More recently, Lindblom-Ylänne and Lonka (1999) used a combination of ASI and IL.S and found that meaning orientation was positively correlated with academic performance in a traditional medical curriculum.

In general. several researchers in medical as well as non-medical disciplines have found a relation between learning approach and academic success among university students. A consensus view on this issue seems to be that reproduction orientation is negatively related to study success whereas meaning orientations and self-regulation correlate positively to study success (Beishuizen \& Stoutjesdijk. 1999: Entwistle, Meyer. \& Tait. 1991: Entwistle \& Tait. 1990; I.indblom-Ylänne, 1999: Nhan. 2006; Vanderstoep, Pintrich. \& Fagerlin. 1996: Vermunt. 2005: Vermunt $\&$ Vermetten. 2004). Busato. Prins. Elshout, and Hamaker (1998) concluded that although they were unable to find a positive correlation between meaning directed or application directed learning and academic performance. undirected learning correlated negatively with academic success.

In the present investigation. at the learning style level a negative association between reproduction oriented learning and examination performance was evident only among the third year group of Sri Lankan medical students. It could be that the assessments held at the end of third year demanded deep level cognitive processing. However, at ARPM scale level (i.e. critical processing, memorising and rehearsing, external regulation, etc.) a significant association between the seale scores and examination performance was not evident in any one of the student groups. One possible explanation put forward by some of the researchers for the lack of association between academic performance and meaning orientation among medical students in particular, is that examinations for medical students test knowledge, which requires limited deep-level processing (Newble, fintwistle, Heika, Jolley, \& Welan. 1988: Stiernborg \& Bandaranayake, 19961. The Sri 
Chapter 9

Lankan situation is no exception. Student perception of the present system of assessment as encouraging memorization of facts (chapter 8) is also in support of this argument.

Furthermore, contrary to what was expected, an association between some other personal variables such as parental education, parental occupation (whether the mother is a teacher or not), and district of entry to medical school (privileged/underprivileged), and ARPM scale scores too failed to appear. Among Sri Lankan students, associations between gender, attempt in which university selection is secured and academic locus of control in the one hand, and learning style on the other were not evident. Although research in the past has suggested a negative association between internal locus of control and a surface approach to studying (Drew \& Watkins, 1998) a similar relationship was not evident among the medical students. Similar findings did not suggest that either males or females have a tendency to use one particular type of learning approach. However, Stiernborg and Bandaranayake (1996) reported that two of the ASI-subscales under meaning orientation, comprehension learning and use of evidence, showed significantly higher scores for males. Thus, our results were not in line with their findings. When the results are taken together, those may suggest that Sri Lankan students, similar to the Chinese students, demonstrate their marvellous ability to adapt to a given learning environment, thus the differences in learning can hardly be explained by personal factors alone (Kember, 1996).

Also the associations between the use of different learning activities and average number of hours spent on independent study, as reported by students, were investigated. Although relatively small, a positive correlation was shown with the scales memorizing and rehearsing, external regulation of learning processes, certificate directed orientation and the learning conception intake of knowledge. All these represent a reproduction directed learning style associated with longer study times. Other positive correlations were between relating and structuring and self-regulation of learning contents and study time.

In this respect, Kember, Jamieson, Pomfret, and Wong (1995) investigated the relationship between learning approach, time spent on studying, and grades awarded among a group of engineering students in Hong Kong. They reported that the use of a surface approach to learning was positively correlated with the number of hours of independent study and attributed that to the inefficiency of the surface approach. However, in previous studies with psychology students, Svensson (1977) as well as Parer and Benson (1989) have not found a significant relationship between surface approach and longer study times. Their interpretation was that students who exhibit a deep approach spend longer time on their studies. Among Sri Lankan students a positive correlation between surface strategies and study time was evident and at the same time, a positive correlation between self-regulation of learning as well as relating and structuring processing strategy and study time was also evident. Although there are methodological differences, the Sri Lankan results correspond to these previous research and suggest that Sri Lankan learners use the so called 'surface strategies' and meaning oriented strategies together, which is a feature of a strategic approach, and therefore spend a longer time on independent study.

\section{Cross cultural comparisons}

Going by the comparisons made in chapter 7 , it was evident that the Indonesian group of students, despite the fact they are non-medical students, showed similar learning patterns to Sri Lankan medical students, while the differences in the corresponding domains were greater with Dutch students. This could shed light on the fact that cultural similarities in 
the two Asian countries (discussed in chapter 7) acting through the student and the learning environment as depicted in the model underlying the dissertation (chapter 4), may be resulting in the reporting of similar behaviours. Both Asian groups of students showed considerably higher scores on most of the learning conceptions and orientation scales while this was not the case for the pattern of scores for learning strategies in the Dutch study. For these items, the respondents had to indicate the degree to which they agree or disagree with a statement, as opposed to the part on strategies, in which they had to indicate how frequently they did something. One reason for the emergence of these high scores could be that disagreeing with statements that come from an authority is felt to be more impolite in Asian than in Western culture. On the other hand, Dutch students are very hard to press to use the extremes of scales. Hence, the scale scores may have an element of cultural bias. Although cultural biases may have an effect on comparability of scale mean scores, factor structures will not be affected. These observations are in support of the suggestion that learning experiences are culturally coloured (Marton et al. 1997; Pintrich, Zuscho, Schiefele, \& Pekrun, 2001).

The concept of a "learning style" (Vermunt \& Verloop, 2000) or "learning orchestration" (Meyer, 1991), showing interrelations between the affective, meta-cognitive and cognitive domains were amply demonstrated in this cross-cultural study. Cultures differ in the emphasis they place on various aspects of the learning processes. It could very well be the reason for the expression of more similar trends among first year Asian students than their Dutch counterparts. Internal associations between learning elements were less strong in the two Asian studies. Nhan (2006) made a similar observation on Vietnamese medical students. Concerning dimensions of national cultures, a reference is made to Hofstede (2001), in which he reported the basis for similarities in certain educational practices among certain nations. It can be postulated that the paternalistic culture common to both Asian groups may be responsible for creating a friction between the different components of learning that define a learning pattern. The differences in the learning contexts, students' existing beliefs about learning and learning orientations and task division between students and teachers in the three cultures are important factors that would have given rise to the emergence of this kind of dissonance in the regulation of learning processes among first year students. Absence of a clear, distinct application directed learning pattern and the presence of a passive idealistic learning pattern, characterized by loadings of only one of the learning elements, were the commonalities in the Asian studies. It can be argued that application directed learning becomes more obvious among the advanced students and may emerge in later years among the Asian student groups.

Based on the results of the Sri Lankan and the Indonesian studies one could assume that the emergence of a learning factor with loadings being present only on learning conceptions is a phenomenon peculiar to Asian student groups. Furthermore, as argued in chapter 5 based on the results of Vermunt and Minnaert (2003), it can also be considered a developmental phenomenon in learning. However, the identification of this same factor among all groups of medical students (irrespective of the year of study) makes it less likely to be a developmental phenomenon among the Asians.

All three investigations, which used ILS or one of its adaptations, have repeatedly identified three learning style dimensions among university students. They are the meaning directed style, reproduction directed style and undirected style. It confirms Vermunt's (1998) learning theory, where he described the existence of an interrelationship between different learning elements: cognitive, meta-cognitive and affective. 


\section{The Asian paradox}

The "Asian paradox" is the belief among western researchers that the Asian students engage mainly in rehearsing and memorizing activities without any attempt to understand the content yet frequently outperformed their western counterparts (Kember, 1996). Theoretically, the achieving nature of Asians and the assumption that Asian learners are rote learners, become incompatible, thus giving rise to the Asian paradox.

The interviews with medical students highlighted that Sri Lankan learners, similar to the Chinese and the Japanese learners, combine understanding and memorization, as opposed to their western counterparts (Kember, 1996; Marton et al. 1997, 2005; Purdie et al., 1996). The results of the Sri Lankan interviews strengthen the view that many Asians combine memorizing with understanding (more so in science related fields, where accuracy is important and the assessment system being one of encouraging memorization and recall). Therefore, it must be postulated that the "surface approach" observed by the western researchers among the Asian students is distinct from the western surface approach in which the student has no interest or intention of discovering underlying meaning (Marton et al., 1997, 2005). Hence, it is reasonable to assume that Asian students attempt to understand the content first and then memorize, which is an adaptive strategy these students employ to achieve high grades at the examinations.

Many researchers have investigated the conceptions of learning and learning activities of Chinese, Nepalese, South-east Asian and Japanese students and challenged the belief that Asians are more prone to rote learning (Volet, Renshaw, \& Tietzel, 1994). The results of Tang (1991) as well as Kember and Gow (1990) suggest that Chinese university students do not simply rote learn unprocessed information. Hence they made a distinction between mechanical memorisation and memorizing of understood, deeply processed material merely for high achievement, which led to the description of a 'narrow approach' or a 'deep memorising' approach.

It is also more likely that the Sri Lankan medical students engage in a more elaborative type of surface approach (not the surface approach originally derived from the studies in European publication), characterized by a combination of deep processing strategies and memorizing and rehearsing activities. Therefore, the results revealed associations between all those elements and study time. The Sri Lankan results are in favour of the argument that the learning approaches of Asian students cannot be adequately described by the deep - surface dichotomy alone. The results suggest that Sri Lankan medical students seek understanding, and explicitly try to commit pertinent information to memory, spending a considerable amount of time, since they believe that such a combined approach leads to high achievements within the existing learning context. It is also evident that the ethnic Chinese have been successful within higher education in Australia and the USA ( Kember, 1996; Marton et al. 1997) which demands deep level learning. The existence of the paradox of Asian students, which is being widely observed as attempting to memorise course material yet perform well academically, can be attributed to the propensity of the Asian students to combine memorizing and understanding.

At the same time comparison of factor structures of Dutch and Sri Lankan students showed among other things that Dutch students experience aspects of learning patterns as separate that for Sri Lankan students can go well together. This resembles the results of studies of Ajisuksmo and Vermunt (1999) and Marton et al. (1997). It also suggests the possibility that Sri Lankan students' learning activities are more under control of the learning environment. Despite the belief among academics in western countries that 
Asian students rely heavily on rote learning and memorization, it has been pointed out by several researchers that these students do not score higher than the western students on the surface learning approach (Volet et al., 1994). The results of the present study endorse this view; the anecdotal claim of "Asians are inherently inclined to be rote learners" is not supported by serious empirical evidence (Kember \& Gow, 1991).

\section{Developmental trends in learning patterns}

The cross-sectional study as well as the longitudinal study (using the adapted version of the ILS) described in chapter 8, failed to provide evidence that the Sri Lankan medical students' learning patterns become more meaning-directed during the course of their tertiary education. In other words, the present investigation failed to reveal an emerging trend of developing desirable learning patterns, which consist of deep level cognitive processing strategies and coherent linkages with the components of learning, such as meaning or application directed learning with academic progress during the MBBS course. On the contrary, it did reveal diminishing personal interest among students, a factor that needs to be explored at length. In addition, generalization of results requires its replication with another cohort going up to the final year. Nevertheless, the findings should ring an alarm to the curriculum developers.

The literature revealed that student oriented learning environments with increased levels of activating instruction, such as group discussions and assignments, promote the development of meaning oriented learning compared to traditional teacher centred environments (Vermetten, 1999) Vernon and Blake (1993), in their meta-analysis of evaluative research on problem based learning, indicated that PBL programs place more emphasis on meaning (understanding) than on reproducing (rote learning and memorization), and that the opposite pattern prevailed in traditional curriculum. Our results support the argument that the traditional curriculum does not appear to be promoting constructive learning among the average learners. At an earlier instance, Stiernborg and Bandaranayake (1996) found that in a cross sectional design medical students did not show a significant increase in meaning orientation, though they showed a clear increase in surface orientation up to the fourth year. Several researchers have indicated that medical students, learning in traditional curricula, have not indicated greater use of meaningful learning strategies with academic advancement (Coles, 1985; Lindblom-Ylänne, 1999; Newble \& Clarke, 1986; Tooth, Tonge, \& McManus, 1989; Tan \& Thanaraj, 1993). Recently, Nhan (2006) reported that in a cross sectional study final year medical students reported significantly higher scores for deep processing activities, self-regulation and use of knowledge along with stepwise processing, intake of knowledge, ambivalent and certificate directed orientations than the pre clinical students. Thus, our results confirm previous research and add to the mounting evidence that conventional curricula foster approaches to learning that are less desirable (Stiernborg \& Bandaranayake, 1996; Vernon \& Blake, 1993). The fact that the present investigation failed to demonstrate an increase in the use of selfregulation in learning among advanced medical students is consistent with the findings of previous research, which suggested a greater degree of independent study in PBL programs than in traditional programs (Vernon \& Blake, 1993).

Furthermore, several longitudinal studies, which have addressed this issue within a student oriented learning environment (among Dutch university students in the faculties of humanities), reported a significant increase in deep approach scores over a period of one to two semesters (Vermetten, Vermunt, \& Lodewijks, 1999a; Vermunt \& Minnaert, 
2003). Kember (1996) argued that if all the higher education institutes uphold the espoused theories, the learning environments in higher education should be somewhat similar. However, in reality it is not always the case and great cultural and disciplinary variations are evident (Vermunt, 2005). In explaining the findings of the Sri Lankan study one need to be cognizant of the fact that the existing institutional culture of most of the Asian higher education institutes are different to those in the western countries, and to a reasonable extent is coloured by the national culture (Hofstede, 2001).

With respect to the changes in high positive and negative loadings on corresponding factor structures observed during the third MBBS stage, it was expected that according to the developmental hypothesis (Vermunt \& Vermetten, 2004) as well as the Model of Domain Learning (Alexander, Jetton, \& Kulikowich, 1995), the factor structures would strengthen during this period of the course. However, as shown in this study, the factor structures seemed to disintegrate as the student group advanced by five academic terms during the third MBBS course. Therefore, the context hypothesis is brought in to explain the changes in the factor structure during this period. Vermetten et al. (1999a) in their study documented that the factor structure was less clear in the 1st year of studies than in the 2nd year. Recently, Vermunt and Minnaert (2003) also documented a breakdown in factor structure among a group of Belgian sociology students in the third trimester within a student oriented learning environment. Previously, a comparison of the factor structures (Roosendaal \& Vermunt, 1996, as cited by Vermunt \& Vermetten, 2004) in the upper phase of secondary education with those in the first year in university revealed that the factor structure seemed to fall back to pieces. Both unclear factor patterns herald a period of new type of education or a new educational context. This may indicate a period of "friction", a period during which students find that ideas of knowledge and how to go about learning are no longer adequate (Vermunt \& Verloop, 1999). Thus, the breakdown in factor structure, among the advanced students can be explained as follows. Medical students following a traditional curriculum during the $3^{\text {rd }}$ MBBS period have to study a number of para-clinical subjects, attend clinical clerkships, and are subjected to many assessments. Overloaded curriculum, frequent examinations, clinical clerkships all may be adding on to the extra volume of work and thereby making learning extremely stressful.

Research evidence in the past suggests the presence of consistency as well as variability in the use of learning strategies, conceptions of learning and learning orientations with academic progress and contextual variation (Busato et al. 1998; Vermetten, 1999; Vermunt \& Vermetten, 2004) at group level. All the Dutch studies have reported testretest correlations in the range of $.40-.80$ (Vermunt \& Vermetten, 2004). However, personal consistency was not so much a feature among Sri Lankan medical students. For the third year group of students, after five terms of academic studies, all the test-retest correlations appeared to be between .23 and .49. Among the learning strategies, memorizing and rehearsing and lack of regulation were the most stable. Vermetten, Lodewijks, and Vermunt (1999b) also reported that memorizing and rehearsing was relatively stable. Lack of regulation was the least stable among learning strategies in the Dutch study. With respect to the issue of consistency and variability in the use of learning strategies, learning orientations and conceptions of learning across time and contexts among first year medical students, most of the test-retest correlations were not significant (chapter 8).

The findings of the longitudinal study described in chapter 8 also suggests that unlike in Vermetten et al. (1999a) study, the rank order of students on different learning strategies has changed considerably over time, particularly among first year students. It may be that the changes in the learning environment from senior secondary to university 
type with less external support, affected different students in a different manner. Results presented in chapter 8 consolidates the position adopted by Vermunt and Vermetten (2004), in that the question of variability versus consistency of learning strategies, conceptions of learning and learning orientations over time or across different courses yields an 'and and' answer as opposed to an 'either or' answer.

Based on the results of third year group of students it can be suggested that learning strategies appear to be relatively stable compared to learning conceptions and orientations. These results are in line with the results of Vermetten et al. (1999a). Thus, both studies failed to confirm the model proposed by Vermunt (1998), in which learning strategies are considered as less stable than the orientations and conceptions of learning. As suggested by Vermetten et al. (1999a), perhaps the more general and abstract nature of the statements, in the latter two variables would have made it difficult for the students to answer and that would have caused greater variation. It could also be that Sri Lankan students only started to think about some of these aspects more seriously at time 2 after experiencing the learning context, thus the scores appeared to have changed considerably.

Had there been strong personal consistency, there would certainly be a place for entrance examinations as a means to deal with individual differences. However, since learning elements seem to show a wide variation across time and across contexts, as was shown in this study, it indicates that strategies are possibly susceptible to modification by training. Furthermore, the adaptable nature of learning strategies, conceptions and orientations to contextual variation offer a chance of success for programs such as process oriented learning or problem based learning (Vermunt \& Vermetten, 2004), which aim at teaching learning strategies in coherence with domain knowledge. All in all the results of the Sri Lankan study too suggest the possibility of existence of a context bound component and a person bound component in the use of learning strategies, conceptions of learning and learning orientations as depicted in the conceptual model, which implies that attempts to change the learning processes of students requires considerable amount of effort and time and thus cannot be realized in a day or two.

\section{Students' perceptions of the existing learning environment}

In order to identify the possible contextual factors that may promote the observed changes in learning behaviours among Sri Lankan medical students, course perceptions of the first year and the fourth year medical students were assessed by means of the Course Experience Questionnaire (Ramsden, 1998). Remarkably, 96\% of the first year students and $89 \%$ of the fourth year students, reflecting on their learning environment during the $2^{\text {nd }}$ MBBS and the third MBBS courses respectively, expressed the view that the "workload was too heavy". The majority also indicated that the learning objectives lacked clarity, guidance and direction was not adequate, there was an over reliance on memorisation of facts, and the time given to understand the subject content was not adequate.

Based on these perceptions, it is reasonable to assume that the learning environment at the faculty of Medicine, University of Peradeniya is more of a teacher centred one with an overloaded curriculum. These perceptions are interpreted in the light of results of previous research, which have investigated the associations between learning approaches and contextual factors. In this respect, researchers have found that perceived heavy workload and less freedom in learning are related to a surface approach to studying (Dahlgren, 1997; Entwistle \& Ramsden, 1983; Trigwell \& Prosser, 1991). The empirical evidence available on the subject is very much in favour of the view that students' experiences of 
teaching and assessment influence their approaches to learning directly and indirectly (Ramsden, 1997; Trigwell \& Prosser, 2004).

On this same issue, Volet et al. (1994) compared the study approaches of South-east Asian and Australian higher education students when the academic context and the course of study were the same. They argued that study approaches are influenced by students' perceptions of course requirements rather than determined by stable personal characteristics of individuals or cultural differences.

As stated in chapter 8 the results of the longitudinal studies are not in favour of the development of a meaning directed learning pattern among medical students of the FMUP. That is, we did not find a significant increase in the use of deep processing strategies, self-regulation strategies or a significant increase in personal interest or construction of knowledge scores with academic advancement. Therefore, it may be reasonable to assume that the student perceptions of the learning environment as encouraging memorization, overloaded curriculum, inadequate teacher guidance during learning difficulties and somewhat unclear course objectives are exerting a negative influence on the development of a more constructive learning pattern. When the perceptions and learning strategies are compared, the results of Sri Lankan studies are in support of Genn's (2001, p.337) view that "the perceived learning environment is an important determinant of the learning behaviour of students." Thus, the above findings corroborate the assumption made in the model underlying the dissertation that student perceptions of the learning environment play a central role in the quality of learning accomplished.

\section{Implications for educational practice}

The fact that educational strategies adopted by traditional schools do not seem to be successful in encouraging the students to use a more desirable meaning directed approach, was repeatedly seen during the third and fourth years of the course, gives weight to the argument that the learning context in question is not supportive for the development of desirable attributes. It also seemed that students are able to adapt and develop their study habits and this has important educational implications. Newble and Entwistle (1986) stated with evidence that the traditional curriculum structure, teaching techniques and particularly the assessment methods are more likely to hinder the development of intellectual attributes sought by all medical schools than to support them. Therefore, they challenged the whole basis of the educational approach in medical schools at that time. Moreover, a number of previous studies as well as the present study, though the learning dimensions were measured using indirect means, confirm the hindrance caused by the educational context on improving the quality of learning. Therefore, it is time to consider making substantial changes encompassing the recommendations of General Medical Council (1993). However, with respect to change, as pointed out by Newble and Clarke (1986) it is unlikely that an established school will be able to introduce the degree of innovation which was possible for PBL schools, most of which were new institutions. It seems that students are able to adapt and develop (at least to some extent) their way of learning. Had we found only a strong degree of individual consistency in learning, then entrance examinations would have been a more appropriate way of dealing with individual differences. The fact that our study revealed a considerable degree of variation of certain learning elements, at individual level, within the first three terms as well as in the third year of the course, has important educational implications, indicating a chance of success for programs aimed at improving the quality of learning outcomes. 
Instructional efforts explicilly guiding students in the development of learning strategies and learning conceptions such as process oriented instruction (Volet, McGill, \& Pears, 1995), activating instruction (Lonka \& Ahola, 1995), problem based learning (Barrows, 1998), or at least incorporation of some of those instructional features into an existing curriculum would no doubt improve the quality of learning and the learning results of students. It is emphasized that students learn how to learn by practicing how to do it. Thus, the teacher has to pay attention to providing opportunities for active learning and avoid too many teacher directed classes. Moreover, empirical studies indicate some kind of active learning is especially helpful to the average student because students employ active learning to a great extent on their own accord (Van Hout-Wolters, Simons, \& Volet, 2000). As speculated by Van Hout-Wolters et al. (2000), one of the reasons for lack of active learning in some institutions may be that it has clearly not become the spearhead of policy.

Results of the present study confirm the findings of Vermetten, Lodewijks and Vermunt (1999b) and Vermunt and Vermetten (2004) that student learning patterns should be considered as changeable phenomena. Since several intervention studies have revealed the possibility of changing student learning patterns in a desirable direction with altering the approach to teaching in the institution (of course not in a day or two) it seems reasonable to argue for programmes such as "process oriented instruction" (Vermunt \& Verschaffel, 2000) or problem-based learning (Barrows, 1998) in which domain specific knowledge is taught in coherence with learning strategies. However, it must be emphatically stated that the faculty concerned should play a leading role in revising its curriculum (outside expertise may be obtained at various stages of the process), and decide on their educational policy considering the resources available and what could be developed to suit the students as well as national needs.

Since strong teacher control tends to inhibit development of constructive learning processes among students, a shared control is proposed in making decisions regarding learning (Vermunt \& Verloop, 1999; Vermunt \& Verschaffel, 2000) be practiced as the educational philosophy of the institution. This means that the teachers guide the students to some extent, but also some responsibility is laid in the hands of the undergraduate themselves with respect to acquiring various skills. Thereby, the students are made to practice intellectual skills that they are not familiar with. An example of teacher created constructive friction is to ask students who are used to memorising to discuss the arguments for and against a certain point of view after critical processing has been demonstrated to the students in some way. According to Vermunt and Verschaffel (2000), constructive friction is necessary to make students willing to change. Constructive friction in a way helps to make students realize that their habitual ways of learning and thinking no longer suffice and leads to activation of skills that are not mastered by them. Thus the net effect is a qualitative improvement in learning activities. On the other hand, going by the cultural background and the school upbringing it is also not advisable to recommend an approach of loose control at the onset of undergraduate education since the chances of failure are expected to be reasonably high.

\section{Directions for change}

The sort of changes which might be possible to improve the situation in a conventional medical school, as pointed out by Newble and Clarke (1986), are a substantial reduction in didactic teaching and work load, an increase in the use of independent learning activi- 
ties and a change in examination methods away from multiple choice questions. Although the results of the present study need to be interpreted with a certain degree of caution, it is recommended that adequate attention be given to the fact that the present curriculum is not actively promoting the development of more constructive learning strategies, such as relating and structuring, concretizing, critical processing and self-regulation. If the results are interpreted as an indication of certain features of the learning environment, such as the increase in lack of regulation and ambivalence and the decrease in personal interest, selfregulation, concrete processing and relating and structuring strategies are attributed to overloaded curriculum and didactic teaching and prevailing assessment system. At the same time a case is made for the consideration of introducing a process-oriented instruction.

Generally speaking Sri Lankan universities do not give sufficient attention to the learning process. This is also evident from the fact that this is the first study, which has looked into qualitative developments in students' learning. The situation is changing no doubt within Sri Lanka itself for the better, with curriculum reforms in higher education occupying an important position in the reform agenda (UGC, 2004b). However, greater attention for the educational function of universities is necessary and our weaknesses need to be investigated and corrected. Having identified some of the drawbacks of the prevailing system at the Peradeniya medical school, it is the right time to propose remedial measures. However, one must not disregard the fact that these changes in instruction requires a high degree of commitment of all parties concerned and take considerable time and effort for its successful implementation (Vermetten, Lodewijks, \& Vermunt, 2002; Vermunt, 1998).

As a first step the faculty needs to open up a dialogue between the staff, students, and administrators on the teaching learning process. Based on the outcome it is advisable to draw out a joint plan to make the learning environment more conducive for active learning among students. Although the learning environment is not a major concern of planners, one needs to emphasize its powerful influence on the quality of learning outcomes. The effect of learning environment on student learning has been emphasised extensively by previous researchers (Ramsden 1997; Vermetten, 1999; Vermunt \& Vermetten, 2004; Vermunt, 2005). In our investigation on course experiences of first year as well as fourth year medical students (as revealed in chapter 8) a majority endorsed the idea that "the workload was too heavy "and "examiners emphasised too much on factual recall". A majority of the students expressed the view that they were not satisfied with the organization of the course. Most of these are contextual factors that were shown to encourage a surface learning approach (Bloomfield et al., 2003; Dahlgren, 1997; Entwistle \& Ramsden, 1983; Trigwell \& Prosser, 1991).

Furthermore, as was pointed out in chapter 8 , examinations dominate the lives of medical students. It is unarguable that assessment procedures used have a powerful influence on learning. "To change curricula or instructional methods without changing the examinations will achieve nothing! Changing the examination system without changing the curriculum had a much more profound impact upon the nature of learning than changing curriculum without altering the examination system." (Miller, 1976, p.79). Therefore, one needs to critically review existing assessment systems with a view to making feasible changes in order to achieve qualitatively better learning outcomes.

Students should be encouraged to present their point of views in seminars, discussions, etc. in the presence of tutors so that whatever misconceptions they have can be corrected. Also active self-directed learning among students must be encouraged as far as 
possible within the course. It is also essential that over-reliance on a large quantity of facts during the course is discouraged by shift in the emphasis placed on recall of facts during examinations. Moreover, introducing incourse-assessments and other forms of assessments will encourage more meaningful learning. As depicted in the (chapter 4) conceptual model, the beliefs, goals and conceptions of both students and teachers influence learning behaviour. Thus, awareness and critical reflection are assumed to be prerequisites for change (Vermetten, 1999). Therefore, it is imperative that instructional measures address the conceptual domain of learning, and attempt a critical reflection of the learner's conceptions and beliefs with respect to learning.

These students' perceptions about the learning environment, together with developmental trends shown by the participant students, makes one question whether the environmental influences have played an inhibitory role in the learning process. The learning patterns we saw might well be a reflection of the learning environment. Successful implementation of any educational innovation is in the hands of the respective teachers (Trigwell \& Prosser, 2004). Students identify teachers as being both help and a hindrance to their learning (Caplow, Donaldson, Kardash, \& Hosokawa, 1997). Therefore, staff development activities in areas where the staff seems to be deficient need to be carried out according to the institutional policy.

In proposing changes one needs to be conscious of the available resources; manpower as well as infrastructure facilities and factors such as the number of students per batch, the cultural upbringing, the educational background of students, etc. However, as revealed by both batches of students there appear to be a problem of work overload in the existing curriculum. In this respect several research studies undertaken in the past have also shown that discipline based curricula are overloaded with information, some of which is irrelevant and most of it is forgotten (Jayawickramarajah, 1995). This issue needs to be looked at carefully and appropriate remedial measures need to be taken as a priority. It seems advisable for the faculty to draw up a core curriculum taking into consideration the societal needs and aspirations (General Medical Council, 1993). The faculty also needs to rethink its present instructional methods as well. Particularly, the effectiveness of lectures as a mode of instruction in promoting active learning among students should be questioned. Considering the advantages of problem based learning in the area of relevance and also its superiority over a conventional curriculum in enabling the students to retain a greater proportion of what is learnt, it is recommended that this tool is also included in the teachers tool kit, along with other methods rather than used as the sole educational strategy (Davis \& Harden, 1999). It is also advisable to base the curriculum reform process on the general principal of PBL; students are not regarded as passive vessels to be filled with facts by lecturers, but that they actively learn for themselves under the guidance of the teacher or instruction.

The infrastructure as at present, the characteristics of enrolled students, and large numbers of students being admitted per academic year, are factors that are not conducive to introduce radical changes. Though a methodical analysis has not been conducted, the general feeling among most of the faculty is that PBL is not going to be as effective as the conventional curriculum in the context of this particular medical school. Among the expressed fears are: that it leads to the creation of gaps in knowledge; logistical problems particularly with more than 170 students in a batch; staff not being adequately trained to handle this approach to learning; lack of cultural acceptance of this kind of a teaching approach; lack of resources for independent study; "students will not be able to grasp the core without sufficient directive guidance" such as what is repeatedly done in lectures; 
and group learning may not be successful because not all students are cooperative. These constraints need to be taken into account in any revision process, no doubt.

Harden (2000) stated, despite the fact that evaluations of PBL have yielded ambiguous results, the approach has become widely accepted and many traditional schools have changed. Furthermore, available data favoured PBL for two outcome areas: it is considered to be more nurturing and enjoyable by the students, while faculty satisfactions seem to be superior and more importantly, desirable study behaviours seem to be promoted (Albanese \& Mitchell, 1993; Vernon \& Blake, 1993). Recently, Blake, Hosokawa and Riley (2000) added that major PBL revisions did not appear to compromise the performances of medical students on licensing examinations and speculated they may have contributed to higher scores.

All in all it may be beneficial to base the curriculum design on the philosophy 'less is more' that is, be guided by the idea, learning a few important ideas and concepts well is educationally much more powerful than learning an extensive amount of superficial facts (Lindblom-Ylänne, 1999; Resnick \& Collins 1996). This way one could also find the time and resources to change the current instructional methods and create powerful learning environments, which support students' active construction of knowledge.

Finally, it is high time that the Faculty of Medicine at Peradeniya follows some kind of a guideline emerged from studies carried out elsewhere and initiate a process of reviewing and reforming the curriculum on a regular basis, with a view to overcome the existing weaknesses and consolidate its strengths. Any change must first be justified. Change for the sake of change should not be attempted. Justification may be provided by research evidence. In terms of promoting desirable changes in learning approaches, the existing curriculum appears to be lagging behind. Therefore, it is stressed that the curriculum developers pay greater attention to issues such as curriculum overload, development of reasoning skills and current system of assessment during the reform process.

\section{General indications for future research}

A major limitation of the empirical studies reported in this dissertation relates to the use of self-report instruments. However, this is an accepted practice in educational research. Nevertheless, the methodological issue that deserves mentioning here is that no real learning strategy or goal orientations were measured, but the student's own perceptions of these variables. Since it is almost impossible to observe such variables directly, indirect measures will always be necessary. However, the results of some of the empirical studies could have been validated, had we used multiple assessments to obtain converging evidence of the variables (see e.g. Lindblom-Ylänne \& Lonka, 2000).

In the present study we could only include the first year, third year and fourth year students. Final year medical students were a difficult group to contact, thus, only the developments within a particular stage of the MBBS course of studies were investigated. An important question raised is whether these changes are specific for this period of study or whether they will continue throughout the course. The situation may again change in the final year where the students actually apply their knowledge to solve real clinical problems. In addition, the workload in terms of theory knowledge becomes much less during this period and more than anything else, the students see the relevance of what is learnt. It is recommended that a similar study be conducted during the final year in the future to report these changes. Therefore, in the future final year students should also be included in the research design and changes during a longer time span should be ana- 
lyzed. Future research needs to address these questions. It is useful to study the learning behaviours of a cohort of students from the beginning of the first year to the end of the final year.

The influence of course perceptions of students on their learning behaviour is an area that needs to be studied in detail at the individual level. In this respect, the validity of some of the items of the Course Experience Questionnaire (CEQ) that was used among the current group of students (described in chapter 8) is questionable. Therefore, a culturally valid instrument needs to be developed in future research.

The issue of consistency and variability of the learning strategies, learning orientations and conceptions of learning need to be explored further throughout the academic course in a longitudinal design. Furthermore, the decreasing trend of attributing academic failure to effort that was evident in the cross sectional study also needs to be investigated in a longitudinal way.

Another important direction for future research would be to study the changes in learning behaviour upon instructional changes. Since the instructional changes take a long time to have a reasonable impact on the learning process, they need to be studied over a longer period of time. Most of the changes in the learning strategies and interrelations between the four learning components were studied at group level. However, it may also be informative to study changes at subgroup level e.g. high achieving, average and low achieving. There is also a place for studies on the effect of all kind of instructional measures and variables on learning patterns, learning outcomes measures, derived from theory on activating instruction or PBL.

Comparison of the learning patterns (using the ILS) of groups of higher education students coming from different cultural backgrounds, when the academic context and the course of study are the same, would no doubt be helpful to better understand the phenomenon of dissonance in student learning patterns. 


\section{References}

Ajisuksmo, C. R. P. (1996). Self-regulated learning in Indonesian higher education. Doctoral thesis, Tilburg University, The Netherlands \& Atma Jaya Catholic University. Indonesia.

Ajisuksmo, C. R. P. \& Vermunt. J. D. (1999). Learning styles and self-regulation of learning at university: An Indonesian study. Asia pacific Journal of Education, 19, 45-59.

Albanese. M. A. \& Mitchell. S. (1993). Problem-based learning: a review of the literaturc on its outcomes and implementation issues. Academic Medicine, 68, 52-81.

Albaili. M. A. (1997). Differences among low, average and high-achieving college students on learning and study strategies. Educational Psychology, 17, 171-177.

Alexander, P. A., Jetton, T. L., \& Kulikowich. J. M. (1995). Interrelationship of knowledge, interest, and recall: assessing a model of domain learning. Journal of Educational Psychology, 87, 559-575.

Alexander. P. A., Murphy, P. K., Woods. B. S., Duhon, K. E., \& Parker, D. (1997). College instruction and concomitant changes in students knowledge, interest, and strategy use: A study of domain learning. Contemporary Educational Psychologv, 22, 125-146.

Ames, C. (1992). Classrooms; goals, structures and student motivation. Journal of Educational Psychologv, 84, 261-271.

Arnold, I.. \& Feighny, K. M. (1995). Students' general learning approaches and performances in medical school: a longitudinal study. Academic Medicine, 70. 715-722.

Balasuriya, C.. Dharmawardena, D., Rajapakse, L., \& Jayasinghe, S. (2000). Approaches to learning and examination performance. Proceedings of Sri Lanka Medical Association $113^{\text {th }}$ Academic Sessions. 22-25 March, (pp.27-28). Colombo, Sri Lanka: SLMA.

Bandura. A. (1986). Social foundations of thought and action: A social cognitive theory. Englewood Cliffs, NJ: Prentice- Hall.

Barrows, H. S. \& Tamblyn, R. M. (1976). An cvaluation of problem- based learning in small groups utilizing a simulated patient. Journal of Medical Education. 51, 52-54.

Barrows, H. S. (1998). The essentials of Problem-based learning. Journal of Dental Education. 62, 630633.

Beaty, L., Gibbs, G., \& Morgan, A. (1997). Learning orientations and study contracts. In F. Marton, D. Hounsell \& N. Entwistle (Eds.), The Experience of Learning (2 ${ }^{\text {nd }}$ ed.)(pp. 72-86). Edinburgh: Scottish Academic Press.

Bedna, A. K., Cunnigham, D., Duffy, T. M., \& Perry, J. D. (1991). Theory into practice: how do we link. In G. J. Anglin (Ed.), Instructional technologv: past, present and fitture (pp. 88-101). Englewood, CO: Libraries limited.

Beishuizen, J. J. \& Stoutjesdijk, E. T. (1999). Study strategies in a computer assisted study environment. Learning and Instruction, 9, 281-301.

Beishuizen, J., Stoutjesdijk, E., \& Van Putten, K. (1994). Studying textbooks: effects of learning styles, study task and instruction. Learning and Instruction. 4, 151-174.

Bennett, M. (1977). Response characteristics of bilingual managers to organizational questionnaires. Personnel Psychology, 30, 29-36.

Berry, J. W. (1990). Imposed etics, emics and derived etics: Thcir conceptual and operational status in cross- cultural Psychology. In T. N. Headland, K. L. Pike \& M. Harris (Eds.), Emics and Etics: The insider / outsider debate (pp.84-99). Newbury Park, CA: Sage Publications.

Best, D. L. \& Ruther, N. M. (1994). Cross-cultural themes in development psychology: an examination of texts, handbooks, and reviews. Journal of Cross Cultural Psychology, 25, 54-77.

Biggs, J. B. (1978). Individual and group differences in study processes. British Journal of Educational Psychology, 48, 266-279

Biggs, J. B. (1987). Student approaches to learning and studying. Melbournc, Hawthorn,Vic: Australian Council for Educational Rescarch.

Biggs, J. B. (1991). Approaches to learning in secondary and tertiary students in Hong Kong: some comparative studies Educational Research Journal, 6, 27-39.

Biggs, J. B. (1992). Why and how do Hong-Kong students learn? Using the study process questionnaircs, Education paper 14, Faculty of Education, University of Hong-Kong.

Biggs. J. B. (1993). What do inventories of students' learning processes really measure? A theoretical review and clarification. British Journal of Educational Psychology, 63, 3-19.

Biggs, J. (1996). Enhancing teaching through constructive alignment. Higher Education. 32. 347-364.

Biggs. J. B. (1999). Teaching for quality learning at University. Buckingham: Open University Press. 
Blake, R. L.. Hosokawa, M. C., \& Riley, S. L. (2000). Student performances on step 1 and step 2 of the United States medical licensing examination following implementation of a problem-based learning curriculum. Academic Medicine. 75, 66-70.

Bloom field. L., Harris, P., \& Hughes, C. (2003). What do students want? The type of learning activities preferred by final year medical students. Medical Education. 37. 110-118.

Bockearts, M. (1997). Self-Regulated learning: A new concept embraced by researches, policy makers, educators, teachers and students, Learning and Instruction. 7. 161-186.

Boomer, G. (1982). Negotiating the curriculum. Sydney: Ashton Scholastic.

Bond, M. H. (1988). Invitation to a wedding: Chinese values and economic growth. In D. Sinha \& S. R. Kao (Eds.), Social values and development: Asian perspectives (pp. 197-209). New Delhi: Sage publication.

Boud, D. (1985). Problem-based learning in perspective. In D. Boud (Eds.), Problem- based Learning in Education for Professions. Sydney: Higher Education Research and development of Australia.

Boyle, E. A., Duffy, T., \& Dunleavy, K. (2003). Leraning styles and academic outcome: The validity and utility of Vermunt's Inventory of learning styles in a British higher education setting. British Journal of Educational Psychology. 73, 267-290.

Brown, A. (1987). Metacognition, executive control, self regulation, and other more mysterious mechanisms. In F.E. Weinert \& R.II. Kluwe (Eds.), Metacognition. motivation and understanding (pp. 21 29). Hillsdale, N.J.: Lawrence Erlbaum.

Brown, A. 1., Bransford, J. D., Ferrara, R. A., \& Campione, J. C. (1983). Learning remembering, and understanding. In J. H. Flavell \& E. H. Markman (Eds.), Handbook of child psychology: Cognitive Development vol.3 (pp. 77-176). New York: Wiley.

Brown, J. S., Collins, A., \& Duguid, P. (1989). Situated cognition and culture of learning. Educational Researcher, 18. 32-42.

Bruning. R. H., Schraw G. J., \& Ronning. R. R. (1995). Cognitive psychology and instruction. New Jersey: Prentice Hall.

Busato,V. V., Prins,F. J., Elshout, J. J., \& Hamaker, C. (1998). Learning styles: a cross sectional and longitudinal study in higher education. British Joumal of Educational Psychology, 68, 427-441.

Caplow, J. A. H., Donaldson, J. F.. Kardash, C. A., \& Hosokawa, M. (1997). Learning in a problem-based medical curriculum: Students" conceptions. Medical Education, 31, 440-447.

Central Bank (1998). Economic progress of independent Sri Lanka. Colombo: Central Bank of Sri Lanka.

Central Bank (1999). Annual Report. Colombo: Central Bank of Sri Lanka.

Central Bank (2000). Annual Report. Colombo: Central Bank of Sri Lanka.

Central Bank (2003). Annual Report. Colombo: Central Bank of Sri Lanka.

Central Bank (2005). Annual Report. Colombo: Central Bank of Sri Lanka.

Clarke, R. M. (1986). Students' approaches to learning in an innovative medical school: a cross sectional study. British Journal of Educational Psychology, 56, 309-321.

Coles, C. R. (1985). Differences between conventional and problem-based curricula in their students' approaches to studying. Medical Education, 19.308-309.

Coles C. R. (1990). Elaborated learning in undergraduate medical education. Medical Education, 24. 1422.

Christensen, C. A., Massey, D., \& Isaacs, P. (1991). Cognitive strategies and study habits: an analysis of measurement of tertiary students' learning. British Journal of Educational Psychology, 61. 290-299.

Christopoulos, J. P., Rohwer, W. D., \& Thomas, J. W. (1987). Grade level differences in students' study activities as a function of course characterislics. Contemporary Educational Psychology. 12, 303323.

Curry, L. (1983). Learning style in continuing medical education. Ottawa, Ontario, Canada: Canadian Medical Association,

Davis, M. H. \& Harden, R. M. (1999). AMEE Medical Education Guide No. 15: Problem based learning: a practical guide. Medical Teacher, 21,30-140.

Dahlgren, L. O. \& Marton, F. (1978). Students' conceptions of subject matter: an aspect of learning and teaching in higher education. Studies in Higher Education. 3, 25-35.

Dahlgren, L. O. (1997). Learning conceptions and outcomes. In F. Marton, D. Hounsell \& N. Entwistle (Eds.), The Experience of Learning ( ${ }^{\text {nd }}$ ed.)(pp. 23-38). Edinburgh: Scottish Academic Press.

De Silva, C. R. \& De Silva, D. (1990). Education in Sri Lanka 1948-1988. New Dellhi: Navrang.

De Silva L., Babapulle C. J., Marambe, K., \& Edussuriya, D. (2001). The English language ability of fourth year medical students and their performance in the essay component of the forensic medicine 
paper. Proceedings of Anmual Research Sessions University of Peradeniva Sri Lanka. (pp.92) Kandy: University of Peradeniya.

Dolmans, D. H .J. M. \& Wolflagen, I. H. A. P. (2004). The relationship between learning style and learning environment. Medical Education. 38, 800-801.

Dornan, T., Hadfield, J., Brown, M., Boshuizen, H., \& Scherpbier, A. (2005). How can medical students learn in a self-directed way in the clinical environment? Design-based research. Medical Education. 35, 356-364

Drew, P. Y. \& Watkins, D. (1998). Affective variables, learning approaches and academic achievement: a causal modelling investigation with Hong Kong tertiary students. British Joulnal of Educational Psychology, 68, 173-188.

Duch, B. J. (1995). Newsletter of the centre for Teaching effectiveness, University of Delaware. "http://www.udel.edu/pbl/cte/jan95-phys.html"

Duffy, T. M. \& Jonassen, D. H. (1992). Constructivism: new implications for instructional technology, In T.M. Duffy \& D.H. Jonassen (Eds.). Constructivism and technologv of instruction - A conversation (pp. 1-16). Hillsdale, NJ: Erlbaum.

Earthlrends, (2004). Economics. Retrieved July, 25, 2005 from http://earthtrends.wri. org/searchable_db/index.cfm?theme $=5$ \&variable_ID $=6438$ \&action=select_countries

Eisenberg. N. (1986). Applying student-learning research to practice. In J.A. Bowden (Ed.), Student leaming: research into practice (pp.21-60). Parkville: Centre for the study of Higher Education, University of Melbourne.

Eley, M. G. (1992). Differential adoption of study approaches within individual students. Higher Education, 23, 231-254.

Elliot, J. G. \& Bempechat. J. (2002). The cultural and context of achievement motivation. In W. Damon (Ed.), Learning in culture and context: Approaching the complexities of achievement motivation in siudent learning (pp. 7-26). San Francisco: Jossey-Bass.

Emilia, O. \& Mulholand, H. (1991). Approaches to learning of students in an Indonesian medical school. Medical Education. 25. 462-470.

Entwistle, N. (1991). Approaches to learning and perceptions of the learning environment-Introduction to the special issue. Higher Education, 22, 201-204.

Entwistle, N. (1997). Contrasting perspectives on learning. In F. Marton, D. Hounsell \& N. Entwistle (Eds.), The Experience of Learning ( ${ }^{\text {nd }}$ ed.)(pp.3-22). Edinburgh: Scottish Academic Press.

Entwistle, N. J., Meyer, J. H. F., \& Tait, H. (1991). Student failure: disintegrated patterns of study strategies and perceptions of the learning environment. Higher Edication, 21, 249-261.

Entwistle, N. \& McCune, V. (2004). The conceptual bases of study strategy inventories in higher education. Educational Psychology Review, 16, 325-245.

Entwistle, N. J. \& Ramsden, P. (1983). Understanding Student learning. London: Croom Helm.

Entwistle, N. J. \& Tait, H. (1994). The revised approaches to studying inventory. Edinburgh: University of Edinburgh, Centre for Research into Learning and Instruction.

Entwistle, N. J. \& Waterson, S. (1988). Approaches to learning and lcvels of processing: A comparison of inventories derived from contrasting theoretical bases. British Journal of Educational Psychology, $58.258-265$.

Faculty of Medicine (2000). Hand Book of the Faculty of Medicine, University of Pcradeniya, Sri Lanka.

Flavell, J. H. (1987). Speculation about the nature of development of metacognition. In F. E. Weinert \& R. H. Kluwe (Eds.), Metacognition, motivation and understanding (pp. 21-29). Hillsdale, N.J.: Lawrence Erlbaum.

Fransson, A. (1977). On qualitative differences in learning. IV-Effects of notivation and test anxiety on process and outcome. British Journal of Educational Psychologv, 47, 244-257.

General Medical Council (1993). Tomorrow's doctors. London: GMC.

Genn, I. M. (2001). AMEE Medical Education guide No. 23 (Part 1): Curriculum, environment, climate. quality and change in medical education- a unifying perspective. Medical Teacher 23, 337-344.

Geisler- Brenstein, E., Schmeck, R. R., \& Hetherington, J. (1996). An individual difference perspective on student diversity. Higher Education, 31, 73-96.

Godffey, R. C. (1995). Undergraduate examinations a continuing tyranny. The Lancet, 45, 765-767.

Gow, L. \& Kember, D. (1990). Does higher education promote independent learning?. Higher Education. $19,307-322$

Gow, L. \& Kember, D. (1993). Conceptions of teaching and their relationship to student learning. British Journal of Educational Psychology, 63, 20-33. 
Gunawardena. R. P. (1999). Higher Education Science and Technologv: some thoughts. Peradeniya: Author-published.

Harden. R. M. (2000). Evolution or revolution and the future of medical education: Replacing the oak tree. Medical Teacher. 22. 435-442.

Harden, R. M. (2001). The learning environment and the curriculum. Medical Teacher. 23. 335-336.

Harden, R. M., Sowden, S., \& Dunn, W. R. (1984). Educational strategies in curriculum development; the SPICES model. Medical Education, 18, 284-297.

Hattie, J. \& Watkins, D. (1981). Australian and Filipino investigations of the internal structure of Biggs' new study process questionnaire. British Journal of Educational Psvchologv. 51, 241-244.

Hess, R. D. \& Azuma, M. (1991). Cultural support for schooling: contrasts between Japan and the United states. Educational Researcher, 20, 2-8.

Hilliard, R. 1. (1995). How do medical students learn: Medical students learning styles and Factors that affect these learning styles. Teaching and Learning in Medicine. 7, 201-210.

Ho, D. Y. \& Wu, M. (2001). Introduction to cross cultural psychology. In Adler L..L. and Gielen U.P. (Eds.), Cross Cultural Topics in Psychology (2 ${ }^{\text {nd }}$ ed.) (pp.3-13). U.S.A.: Greenwood Publishing group.

Hofstede, G. H. (2001). Culture 's Consequences: Comparing values. behaviours, institutions, and organizations across nations $\left(2^{\text {nd }} \mathrm{ed}\right.$.)(pp. 1-77). California: Sage publications.

Hounsell, D. (1997). Understanding tcaching and teaching for understanding. In F. Marton, D. Hounsell \& N. Entwistle (Eds.), The Experience of Learning ( $2^{\text {nd }}$ ed.) (pp.238-257). Edinburgh: Scottish Academic Press.

Jayasinghe, S. (2002). Reforming a medical culriculum: Lessons, from an established medical school in Sri Lanka. Colombo: Author-published.

Jayawickramrajah, P. T. (1993). Impact of Problem-Based medical curriculum on student's approaches to learning. Bahrain Medical Bulletin. 15, 116-121.

Jayawickramarajah, P. T. (1995). Problem-based learning: an educational strategy for better health care. Trends in Medical Education. 2, 12-15.

Johnson, D. W. \& Johnson, R. T. (1994). Learning together and alone: cooperative, competitive and individualistic learning $\left(4^{\text {th }}\right.$ ed.) Boston : Allyn and bacon.

Kember, D. \& Gow, L. (1990). Cultural specificity of approaches to study. British Journal of Educational Psychology, 60, 356-363.

Kember, D. \& Gow, L. (1991). A challenge to the anecdotal stereotype of the Asian student. Studies in Higher Education, 16. 117-128.

Kember, D. (1996). The intention to both memorise and understand: another approach to learning? Higher Education. 31, 341-354.

Kember, D., Jamieson, Q. W., Pomfret, M., \& Wong, E. T. T. (1995). Learning approaches, study time and academic performance. Higher Education. 29. 329-343.

Kaufman, D. \& Mann, K. V. (1997). Basic sciences in problem-based learning and conventional curricula: students' attitudes. Medical Education. 31, 177-180.

Lakshman. W. D. (1998). University education in Sri Lanka at the golden jubilee of the country's independence: Achievements, challenges and opportunities. Multi-Disciplinary International Con ference on the Occasion of $50^{\text {th }}$ Anniversary of Independence of Sri Lanka, 23-25 February, University of Peradeniya, Sri Lanka: pp 29 (supplement).

Laurillard, D.M. (1997). Styles and approaches in problem solving. In F. Marton, D. Hounsell \& N. Entwistle (Eds.). The Experience of Learning ( $2^{\text {nd }} \mathrm{cd}$.) (pp.126-144). Edinburgh: Scottish Academic Press.

Leiden, L. I., Crosby, R. D., \& Folmer, H. (1990). Assessing learning style inventories and how well they predict academic performance. Academic Medicine, 65, 395-401.

Lonka, K. \& Ahola, K. (1995). Activating instruction - How to foster study and thinking skills in higher education. European Journal of Psychology of Education, 10, 351-368.

Lonka, K., Joram, E., \& Bryson, M. (1996). Conceptions of learning and knowledge: Does training make a difference? Contemporary Educational Psychology, 21, 240-260.

Lonka, K. \& Lindblom-Ylänne, S. (1996). Epistemologies, conceptions of learning and study practices in medicine and psychology. Higher Education, 31, 5-24.

Lindblom-Ylänne, S. (1999). Studying in a traditional medical curriculum- study success, orientations to studying and problems that arise. Doctoral thesis. Helsinki University, Finland 
Lindblom-Ylänne, S. \& Lonka, K. (1999). Individual ways of interacting with the learning environmentare they related to study success? Learning and Instruction. 9, 1-18.

Lindblom-Ylänne, S. \& Lonka, K. (2000). Dissonant study orchestrations of high-achieving university students. European Journal of Psychologv of Education, 15, 19-32.

Marton, F. (1981). Phenomenography- describing conceptions of the world around us. Instructional Science, 10, 177-200.

Marton, F. (1986).'Phenomenography- A research approach to investigating different understandings of reality'. Journal of Thought. $21,28-49$.

Marton, F., Dall'Alba, G.. \& Beaty, E. (1993). 'Conceptions of lcarning'. International Journal of Educational Research, 19. 277-300.

Marton, F., Dall'Alba, G.. \& Tse, L. K. (1992). Solving the paradox of the Asian learner. Paper presented at the Fourth Asian Regional Congress of Cross-cultural Psychology, Katmandu.

Marton, F. \& Saljo, R. (1976). On qualitative differences in learning: I Outcome and process. British journal of Educational Psychology, 46, 4-11.

Marton, F. \& Säljö, R. (1997). Approaches to learning. In F. Marton, D. Hounsell \& N. Entwistle (Eds.),

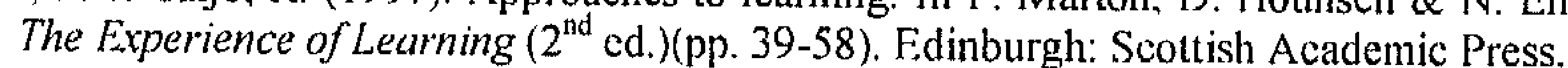

Marton, F., Watkins, D., \& Tang, C. (1997). Discontinuities and continuities in the experience of learning: an interview study of high-school students in Hong Kong. Learning and Instruction, 7. 21-48.

Marton, F., Wen, Q., \& Wong, K.C. (2005). 'Read a hundred times and the meaning will appear... Changes in Chinese university students' views of the temporal structure of learning. Higher Education, 49, $291-318$

Mattick, K., Dennis, I., \& Bligh, J. (2004). Approaches to learning and studying in medical students validation of a revised inventory and its relation to student characteristics and performance. Medical Education. 38. 535 .

Martenson, D.F. (1986). Students' approaches to studying in four medical schools. Medical Education. 20. 532-34.

Meyer, J. H. F. (1991). Study orchestration: The manifestation, interpretation and consequences of contextualised approaches to studying. Higher Education, 22, 297-316.

Meyer, J. H. F. (2000). The modeling of dissonant study orchestration in higher education. European Journal of Psychology of Education, 15, 5-18.

Morgan, A.R., Taylor, E., \& Gibbs, G. (1982) Variation in students' approaches to studying. British Journal of Educational Technology, 13, 107-113.

Miller, G. E. (1976). Assessment in medical school. Medical Education, 10, 79-80.

Minnaert, A. \& Janssen, P. J. (1997). Bias in the assessment of regulation activities in studying at the level of higher education. European Journal of Psychological Assessment, 13, 99-108.

Ministry of Education (2004, January 27). Retrieved May 23, 2006 from http://www.moe.gov.lk

Ministry of Human Resource Development, Education and Cultural Affairs (2004, November 16). Education for all National action plan: Sri Lanka. Retrieved December 20, 2005 from http://portal.unesco.org/education/en/ev.phpURL_ ID =36505\&URL_ DO=DO_TOPIC\&URL_ SECTION=201.hitml

Moran, A. (1991). 'What can learning styles research learn from cognitive psychology?' Educational Psychology. 11, 239-245.

Montecinos, P. B. \& Pantoja, M. A. (1991). The approach to learning in a traditional medical school. Medical Education, 13, 305-310.

Nahn, V. T. (2006). Learning styles of medical students in University of Medicine and Pharmacy at HoChiMinh City. Master thesis, Maastricht University, The Netherlands.

Newble, D. \& Clark, R. (1986). The approaches to learning of students in a traditional and an innovative problem based medical school, Medical Education, 20. 267-273.

Newble, D. \& Clarke, R. (1987). Approaches to learning in a traditional and innovative medical school. In: J. T. E. Richardson, M. W. Eysenck \& D.W. Piper (Eds.), Student learning. Research in educational cognitive psychology (pp.39-46). Great Britain: The sociely for research into Higher education \& Open University press

Newble, D.I. \& Entwistle, N.J. (1986). Learning styles and approaches: implications for medical education. Medical Education, 20, 162-175,

Newble, D. I., Entwistle, N. J., Heika, E. J., Jollcy, B., \& Whelan, G. (1988). Towards the identification of student learning problems: the development of a diagnostic inventory. Medical Education, 20, 162175 . 
Newble, D. I. \& Gordon. M. I. (1985). The learning style of medical students. Medical Education. 19, 3-8.

Nijhuis, J., Segers, M. \& Gijselaers. W. (2005. August). The impact of an educational innovation on students' study strategies and the role of students 'perceptions of the learning environment. Paper presented at the 11 th Conference of the European Association of Research on Learning and Instruction. Nicosia, Cyprus.

Norman, G. R. \& Schmidt, H.G. (1992). The psychological basis of problem-based learning: a review of evidence. Academic Medicine, 67, 557-565.

Oosteerheert. I. E. \& Vermunt. J. D. (2001). Individual differences in learning to teach: relating cognition, regulation and affect. Learning and Instrution. 11, 133-156.

Pask, G. (1976). Styles and strategies of learning. British Journal of Educational Psychologv, 46, 128-148.

Parlett, M. R. \& Hamilton, D. (1972) Evaluation as illumination: a new approach to the study of innovatory programs. Unpublished report. (reprinted in D. Hamilton et al. Beyond the numbers game. Basingstoke: Macmillan, 1977).

Parer, M. \& Benson, R. (1989). Professional training by distance education. Victoria, Australia: Centre for distance learning, Gippsland Institute.

Perera, E. B. P. (1998). An examination of learning Styles of undergraduates in different academic disciplines. Proceedings of the Annual Research sessions, (pp.63). University of Peradeniya, Peradeniya. Sri Lanka.

Perera, L. (2003, May 02). EFA Assessment: country report: Sri Lanka. Retrieved December 20,2005 from http://www2.unesco.org/wef/ countryreports/sri lanka/ contents.html

Perry, W. G. (1970). Forms of intellectual and ethical development in the college years - A scheme. NewYork: Holt, Rinehart \& Winston.

Pieris, N. (2004, November 18). And that made all the difference-innovations and reform to improve quality of education in Sri Lanka. Retrieved May 27, 2005 from htip://portal.unesco.org/ education/en/ev.php-URL_ID $=36144 \& U R L \_D o=D o \_$Topic\&URL_ sections $=201 . \mathrm{html}$

Pintrich, P. R. (2004). A conceptual framework for assessing motivation and self- regulated learning in college students. Educational Psychology Review. 16, 385-407.

Pintrich, P. R., Smith, D., Garcia, T., \& McKeachic, W. J. (1993). Predictive validity and reliability of the motivated Strategies for learning questionnaire (MSLQ). Educational and Psychological measurement, 53, 801-810.

Pintrich, P. R., Zusho, A., Schiefele, U., \& Pekrun, R. (2001). Goal orientation and self regulated learning in the college classroom: A cross cultural comparison. In F. Salili, C. Y. Chiu, \& Y. Y. Hong (Eds.), Student motivation: The culture and context of learning (pp.149-169). Boston: Kluwer academic.

Poortinga, Y. H. (1989). Equivalence of cross- cultural data: an overview of basic issues. International Journal of Psychology. 24, 737-756.

Purdie, N., Hattie, J., \& Douglas, G. (1996). Student conceptions of learning and their use of self-regulated learning strategies: a cross - cultural comparison. Journal of Educational Psychology, 88, 87-100.

Rajapakse, M. (1996). Key note address, Proceedings of the $3^{\text {rit }}$ International Medical Congress 22-26 August, Kandy, Sri Lanka: pp 1-8.

Ramsden, P. (1988). Context and stralegy- situational influences on learning. In R. R. Schmeck (ed.), Learning strategies and learning styles (pp.159-184). New York: Plenum Press.

Ramsden, P. (1992). Learning to teach in higher education. London: Routledge.

Ramsden, P. (1997). The context of learning in academic departments. In F. Marton, D. Hounsell \& N. Entwistle (Eds.), The experience of learning ( $2^{\text {thd }}$ ed.) (pp. 198-216). Edinburgh: Scottish Academic Press.

Ramsden, P. \& Entwistle, N. J. (1981). Effects of academic departments on students' approaches studying. British journal of Educational Psychology, 51, 368-383.

Ramsden, P. (1998). The Course Experience Questionnaire. Melbourne: University of Melbourne

Rayner, S. \& Riding, R. (1997). Towards a categorisation of cognitive styles and learning styles. Educational Psychology, 17, 5-27.

Reid, W. A. (1987). Institutions and practices: professional education reports and the language of reform. Educational Researcher, 16, 10-15.

Rennie, S. (2000). The medical student in the year 2020. Medical Teacher, 22, 532-535.

Resnick, L. B. (1987). Learning in school and oul. Educational Researcher, 16, 13-20.

Resnick, L. B. \& Collins, A. (1996). Cognition and Learning. In E. de Corte \& F. E. Weinert (eds.) International encyclopedia of developmental and instructional psychology (pp. 377-381). Exeter, UK: Pergamon. 
Richardson, J. T. E. (1994). Cultural specificity of approaches to studying in higher education: a literature survey. Higher Education, 27. 449-468.

Richardson. J. T. E. (2004). Methodological issues in questionnaire based research on student learning in higher education. Educational Psychologv Review. 16. 347-358.

Riding, R. I. \& Checma, I. (1991). Cognitive styles- an overview and integration, Educational Psychology. Il, 193-215.

Saljö, R. (1988). 'Learning in educational setting: methods of inquiry'. In P. Ramsden (Ed.), Improving Learning: New Perspectives (pp.32-48). London: Kogan.

Schatteman, A., Carette, E., Couder, J., \& Eisendratl, H. (1997). Understanding the effect of a processoriented instruction in the first year of university by investigating learning style characteristics. Educational Psychologv, 17, 111-125.

Schmidt, H. G., Dauphinee, W. D., \& Patel, V. L. (1987). Comparing the effects of problem based and conventional curricula in an international sample. Joum nal of Medical Education, 62, 305-315.

Schmeck, R. R., Ribich, F. D., \& Ramanaiah, H. (1977). Development of a self- Report inventory for Assessing individual differences in learning processes. Applied Psychological Measurement, 1, 413431

Schunk, D. H. (1991). Learning theories: an educational perspective. New York: Macmillan.

Schommer. M. (1990). Effects of beliefs about the nature of knowledge on comprehension. Journal of Educational Psychologv, 82, 498-504.

Schommer. M. (1998). The influence of age and education on epistemological beliefs. British Journal of Educational Psvchology, 68, 551-562.

Schmeck, R. R. (1983). Learning styles of college students. In R. F. Dillion \& R. R. Schmeck (Eds.), Individual differences in cognition: vol.1 (pp. 233-279). New York: Academic Press.

Schmeck, R. R. (1988). Strategies and styles of learning - an integration of varied perspectives. In R. R. Schmeck (Eds.), Learning strategies and learning styles (pp. 317-347). New York: Plenum Press.

Scott, J. N., Markert, \& Dunn (1998). Critical thinking: change during medical school and relationship to performance in clinical clcrkships. Medical Education, 32, 14-18.

Scnanayake, N. \& Weerasinghc, V. (1996). Does the advanced level aggregate score reflect the subsequent performance of a student in the Medical faculty? Abstracts of research papers. Kandv Society of Medicine, $18^{\text {th }}$ Annual sessions. (pp.38). Kandy:Kandy Society of Medicine.

Severiens, S. (1997). Gender and learning- Learning styles, ways of knowing and patterns of reasoning. Academic dissertation, University of Amsterdam, The Netherlands

Severiens, S. E. \& Ten Dam, G. T. M. (1997). Gender and gender identity differences in learning styles. Educational Psychology, 21, 423-446.

Shuell, T. J. (1988). 'The role of the student in learning from instruction'. Contemporary educational Psychology, 13, 276-295.

Shuell, T. J. (1996). Teaching and learning in a classroom context. In D. C. Berliner \& R. C. Calfece (Eds), Handbook of Educational Psychology (pp.726-764). New York: Simon \& Schuster Macmillan.

Simons. P. R. J. (1997). From romanticism to practice in learning. Life long learning in Europe. 1. 8-15.

Snow, R. E.. Corno, L., \& Jackson. D. (1996), Individual differences in affective and conative functions. In D. C. Berliner \& R. C. Caltee (Eds.), Handbook of Educational Psychology. (pp. 243-310). New York: Macmillan.

Steinert, Y. (1996). Twelve tips for effective small group teaching in the health professions. Medical Teacher. 18, 203-207.

Sternberg, R. J. (1998). Abilitics are forms of developing expertise. Educational Researcher, 27, 11 20.Sticrnborg, M. \& Bandaranayakc, R.C. (1996). Medical students" approaches to studying. Medical Teacher, 18, 229-236.

Strømse, H. I, Grottum, P., \& Hofgaard Lycke, K. (2004). Changes in student approaches to learning with the introduction of computer-supported problem-based learning. Medical Edication, 38, 390-398.

Stokes, M. J., Balla, J. R., \& Stafford, J. (1989). How students in selected degree programmes at CPHK characterize their approaches to study. Educational Research Journal, 4, 85-91.

Svensson, L. (1977). Qualitative differences in learning: III. Study skill and learning. British Journal of Educational Psychology, 47, 233-243.

Svensson, L. (1997). Skill in learning and organising knowledge. In F. Marton, D. Hounsell \& N. Entwistle (Eds.), The Experience of Learning ( $2^{\text {nd }}$ ed. $)(\mathrm{pp} .3-22)$. Edinburgh: Scottish Academic Press.

Tan, C. M. \& Thanaja, K. (1993). Influence of context and preferred learning environments: approaches to studying in physiology. Medical Education, 27, 143-159. 
Tang. K. C. C. (1991). Effects of different assessment methods on tertiary students' approaches to studying. Academic dissertation. University of Hong Kong. Hong Kong.

Taylor, E.. Morgan, A. R.. \& Gibbs. G. (1981). The orientations of Open University students to their studies. Teaching at a Distance. 20, 3-12.

Tckian, A. (1997). An application of the SPICES model to the status of medical curricula in the Eastern Mediterranean region. Medical Teacher. 19. 217-218.

Ten Cate, O., Snell. L.., Mann, K., \& Vermunt, J. (2004), Orienting teaching toward the learning process. Academic Medicine. 79. 219-228.

Tenant, M. (1988). Psychology and Adult Learning. London: Routledge.

Thomas, R. M. (1998). Chapter on interpreting tests, questionnaires and interviews. In R. M. Thomas (Eds.), Conducting Educational Research: A comparative view (pp. 271-295). USA: Bergin \& Garvey.

Thomas, P. R. \& Bain, J. D. (1984). Contextual differences of learning approaches: the effects of assessment Human Learning. 3, 227-240.

Trigwell, K. \& Prosser, M. (1991). Improving the quality of student learning; The influence of learning context and student approaches to learning on learning outcomes. Higher Education, 22, 251-266.

Trigwell, K. \& Prosser, M. (1996). Congruence between intention and strategy in university science teachers approaches to teaching. Higher Education. 32. 77-87.

Trigwell, K. \& Prosser, M. (2004). Development and use of the Approaches to Teaching Inventory. Educational Psychologv Review. 16, 409-424.

Trigwell, K., Prosser, M.. \& Waterhouse, F. (1999). Relations between teachers' approaches to teaching and students' approaches to learning. Higher Education, 37, 57-70.

Tooth, D.. Tonge, K., \& Mcmanus, I.C. (1989). Anxiety and study methods in preclinical students; causal relation to examination performance. Medical Education. 23, 416-421.

UGC (1997). Sri Lankan Universities Year Book 1996. Colombo: University Grants Commission.

UGC (1998). Sri Lanka Universities Year Book 1997/ 1998. Colombo: University Grants Commission.

UGC (2000). Sri Lanka Universities Year Book 1999/2000. Colombo, Sri Lanka: University Grants Commission.

UGC (2003). Sri Lanka Universities Year Book 2002/2003. Colombo: University Grants Commission.

UGC (2004a). UGC statistics. Retrieved May 26, 2006 from http://www.ugc.ac.lk/statistics.litml

UGC (2004b). Sri Lanka Universities Year Book 2003/2004. Colombo: University Grants Commission.

UGC (2004c). UGC admissions. Retrieved May 24, 2006 from http://www.ugc.ac.lk/admission.html

Van de Vijver, F. J. R. \& Poorlinga, Y. H. (1991). Testing across cultures. In R. K. Hambleton \& J. N. Zaal (Eds.), Advances in educational psychological testing: Theory and application (pp. 277-308). Boston: Kluwer.

Vanderstoep, S. W., Pintrich, P. R., \& Fagerlin, A. (1996). Disciplinary differences in self-regulated learning in college students. Contemporary Educational Psychology, 21,345-362.

Van der Vleuten, C. P. M. (1996). The assessment of professional competence: developments, research and practical implications. Advances in Health Science Education, 1, 41-67.

Van Hout-Wolters, B. (2000). Assessing active self-directed learning. In R.J. Simons, J. van der Linden \& T. Duffy (Eds.), New Learning (pp. 83-99). Dordrecht, Boston: Kluwer Academic.

Van Hout-Wolters, B.. Simons, R. J., \& Volet, S. (2000). Active learning: Self-directed learning and independent work. In R. J. Simons, J. van der Linden \& T. Duffy (Eds.), New learning (pp. 21-36). Dordrecht, Boston : Kluwer Academic Publishers.

Van Mil, M. (2005). Verschillen in leerstijlen van Chinese en Nederlandse studenten (Differences of learming styles of Chinese and Dutch students). Master thesis, Wageningen University. Wageningen, the Netherlands.

Van Rossum. E.I. \& Schenk, S.M. (1984). The relationship between learning conception, study strategy and learning outcomes. British Journal of Educational Psychology, 54, 73-83.

Varagunam, T. (1996). Medical Education in Sri Lanka. In P. Abeykoon \& N. Mattock (Eds.), Medical Education in South East Asia (pp. 109-130). New Delhi: World Health Organization.

Vermetten, Y. J. (1999). Consistency and variability of student learning in higher education. Academic dissertation, Tilburg Universily, The Netherlands.

Vermetten, Y. J., Vermunt, J. D., and Lodewijks, H. G. (1999a). A longitudinal Perspective on learning strategies in higher education: Different viewpoints towards development. British Journal of Educational Psychology, 69, 221-242. 
Chapter 10

Vermetten. Y., Lodewijks. H.G . \& Vermunt, J. D. (1999b). Consistency and variability of learning strategies in different university courses. Higher Education, 37, 1-21

Vermetten. Y., Lodewijks. H. G. \& Vermunt, J. D. (2002). Powerful learning environments? How university students differ in their response to instructional measures. Learning and Instruction. 12, 263284.

Vermunt. J.D. (1994). Inventory of Learning Styles in Higher Education. Tilburg: Tilburg University, Department of Education.

Vermunt. J. D. (1996). Metacognitive, Cognitive and affective aspects of learning styles and strategies: A phenomenographic analysis. Higher Education. 31, 25-50.

Vermunt, J. D. (1998). The regulation of constructive learning processes. British Journal of Educational Psvchologv, 68, 149-171.

Vermunt, J. D. (2005). Relations between student learning patterns and personal and contextual factors and academic performance. Higher Education, 49. 205-234.

Vermunt. J. D. \& Verloop. N. (1999). Congruence and friction between learning and teaching. Learning and Instruction, 9, 257-280.

Vermunt, J. D. \& Verloop, N. (2000). Dissonance in students` regulation of learning process. European Journal of Psychology of Education. 15, 75-87.

Vermunt, J. \& Minnaert, A. (2003). Dissonance in student learning patterns: when to revisc theory'? Studies in Higher Education. 28. 49-61.

Vermunt. J. D. \& Verschaffel, L. (2000). Process-oriented teaching. In R. J.Simons, J. van der Linden \& T. Duffy (Eds.), New Learning (pp. 209-225). Dordrecht. Boston: Kluwer Academic Publishers.

Vermunt, J. D. \& Vermetten, V. Y. (2004). Patterns in student learning: relationships between learning strategies, conceptions of learning and Learning oricntations. Educational Psychology Review. 16, 359-384.

Vernon, D. T. A. \& Blake, R. L. (1993). Does problem-based lcarning work? A meta analysis of evaluativc research. Academic Medicine, 68, 550-63.

Volet, S., McGill, T., \& Pears, H. (1995). Implementing process based instruction in regular university teaching: conceptual, methodological and practical issues. European Journal of Psychology of Education, $10,385-400$

Volet S. E., Renshaw, P. D., \& Tietzel, K. (1994). A short term longitudinal investigation of crosscultural differences in study approaches using Biggs' SPQ questionnaire. British Journal of Eucational Psychology, 64, 301-318.

Wagner, E. D. \& McCombs, B. L. (1995). Learner centered psychological principles in practice: design for distance education. Educational Technology, 35, 32-35.

Watkins, D. (1983). 'Assessing tertiary study processes". Human Learning, 2, 29-37.

Watkins, D. (1989). Causal relationships among self concept, attributions, and achievement in Filipino students. Journal of Social Psychologv, 130. 625-631.

Watkins D. \& Akande, A. (1994). Approaches to learning of Nigerian secondary school children: Emic and etic perspectives. International Journal of Psychology, 29, 165-182

Watkins, D. \& Astilla, E. (1984). The dimensionality, antecedents and study method correlates of the causal attribution of Filipino children. Joumal of Social Psychology, 124, 191-199.

Watkins, D. \& Regmi, M. (1992). How universal are student conceptions of learning? A Nepalese investigation. Psychologia, 25, 101-110.

Watkins, D., Regmi M., \& Astilla, E. (1991). The Asian learner as a rote learner stereotype: Myth or reality" Educational Psychology. 11, 21-34.

Weiner, B. (1992). Human Motivation: Metaphors. Theories and Research. California:Sage Publications.

Weiner, B. (1994). Integrating social and personal theories of achievement striving. Review of Educational Research, 64, 557-573.

Weinstein, C. E., Schulte, A., \& Palner, D. (1987). Learning and Study strategies inventory (LASSI). Clearwater, Flor.: H\&H publications.

Woolfolk, A. E. (1998). Educational Psychology ( $7^{\text {th }}$ edition). Boston: Allyn \& Bacon.

World Health Organization (1996). Medical Education in South East Asia. (P. Abeykoon \& N. Mattock (Eds.), New Delhi: World Health Organization.

Zanting, A., Verloop, N., \& Vermunt, J. D. (2001). Student teachers' belicfs about mentoring and learning to teach during teaching practice. British .Journal of Educational Psychology, 71, 57-80. 


\section{Summary}

'Real learning' is an extremely complex phenomenon. As described in the conceptual model underlying this dissertation, learning is influenced by a number of interacting variables. Social cognitive theory emphasizes the role of the personal, behavioural and environmental factors in learning. Many researchers have emphasized the situational nature of learning. Current learning theory assumes that knowledge is situated, being in part a product of the activity, context and culture in which it is developed and used. Furthermore, empirical studies have shown that the quality of knowledge gained by active knowledge construction is better, more accessible, coherent and usable than knowledge acquired by passive means.

According to some researchers, traditional medical schools seem to pay little attention to the influence of the learning environment on student learning. However, it is a well-known fact that despite intellectual homogeneity, a large number of environmental and personality variables affect an individual's behaviour; resulting in great heterogeneity among a student population within any discipline. Unfortunately, students are often taught as if individual differences do not exist. Nevertheless, knowledge about students' learning "patterns", "styles" or approaches" has potential usefulness for teachers in the curriculum development process as well as for adjusting their way of teaching to maximize learning achievement. A literature review revealed that an in depth analysis of learning patterns of medical students has not been carried out in an Asian context. Nevertheless, the results of such studies have great potential in educational decision making and improvement of instructional practices in medical education. Therefore, student learning patterns were considered an area worthwhile for research.

Educational research is viewed here as a careful, systematic attempt to achieve a better understanding of the educational process, with the aim of improving its effectiveness. Hence the researcher's task is to describe more clearly how learning takes place in higher education, and to point out how teaching and assessment affect the quality of that learning. Against this background, it was considered important to explore the learning strategies, conceptions of learning and learning orientations, their interrelations and the possible influences of personal and contextual variables on aspects of learning of Asian medical students within the context of a traditional medical school.

Furthermore, the objective of present day curriculum reforms is basically to improve the quality of learning processes that students realize. It is commonly accepted that development of expertise involves the process of developing domain specific competence in addition to epistemological development and a shift towards more self-regulated and meaning-directed learning. Thus, the emphasis is on how the students learn best as well as what they should learn (the content). This is vital in the face of increasing redundancy of knowledge. At present there is a trend of reforming undergraduate medical curricula all over the world. The Sri Lankan higher education system is also discussing ways of reforming its curricula as well as carrying out staff development activities to promote selfdirected learning among its students.

The present study is also intended to broaden the understanding of the learning process among Sri Lankan medical students and pave the way for more informed decision-making during curriculum development processes. It is expected that these studies, by unravelling relations between the institutional level, contextual factors and students' 
learning approaches and outcomes, may through the weight of evidence give change agents the support they need to convince their academic and administrative colleagues.

The empirical studies described in this thesis attempted to investigate the trends in the development of learning strategies and self-regulation among Sri Lankan medical students, within the context of a traditional curriculum. The overall objective of the project was to explore the learning patterns as well as the effects of contextual and personological factors on aspects of learning of Asian medical students within a traditional curriculum. More specifically, the empirical studies were aimed to answer the following research questions:

1. What are Sri Lankan medical students' study strategies, conceptions of learning, attribution patterns, values, beliefs, expectations of university teaching and learning and study motives?

2. Can the ILS be adapted to form a reliable and valid diagnostic instrument?

3. What are the learning patterns of Sri Lankan medical students?

4. What are the associations between students' use of learning strategies, their learning conceptions and orientations on the one hand and contextual factors, personal factors and academic performance on the other?

5. Does the national culture play a major role in explaining the students' study strategies and the interrelations between the different aspects of learning?

6. What are the trends in the development of learning strategies?

7. What are the students' perceptions of the existing learning environment?

Additionally and more in general, the study attempted to broaden the understanding of the Asian learner. In all, the studies were intended to provide a wealth for information to help educational decision-making and staff development activities.

The first chapter of the thesis gives a general introduction to the whole project and enumerates the main objectives.

The second chapter orientates the reader to the higher education system of Sri Lanka with special emphasis on medical education and its evolution to the present status. It also discusses the strengths and weaknesses of the present system of undergraduate medical education in Sri Lanka.

Chapter 3 gives the theoretical notions that underpin the different empirical studies, which include: current views on teaching and learning; accepted methods of measuring learning aspects in higher education; revelations of empirical studies on the use of learning strategies by higher education students in the fields of Medicine and others, from the West as well as the Asian continent; an account of the role of culture in influencing learning behaviour; and innovative methods of instruction implemented in medical schools such as problem based learning and process oriented teaching.

Chapter 4 displays the conceptual model underlying the dissertation. In a broad sense the project explored the relationships between personological factors (learning orientations and conceptions of learning), the learning environment, national culture and the reported use of learning strategies, among Sri Lankan undergraduate medical students.

The aim of the qualitative study described in Chapter 5 was to identify different categories of description for the four main components of learning: cognitive processing activities, regulation activities, study motives and conceptions of learning. Moreover, interrelations between those components, new entrant medical students' ideas about factors, which had facilitated learning in senior secondary education and about attributes of an ideal student, their expectations about teaching and learning at university and their aca- 
demic locus of control were studied. Thirty-two new entrants were interviewed on a voluntary basis by the researcher. The transcripts of semi-structured interviews were analysed phenomenographically under the major areas stated above. A different approach was taken in the analysis of the conceptions of students of learning and knowledge. The scale described by Lonka, Joram and Bryson (1996) was used as a guideline for the analysis of students' conceptions of learning, while interview excerpts on the students attributions of their academic success and failure were coded on the basis of Weiner's model of attribution. The relation between learning conception, learning strategy, regulation strategy, study motive, and learning expected in the university was investigated by constructing a table using the categories developed earlier to search for distinctive patterns, taking Vermunt's findings on integration between learning conceptions, motives and study strategies as a heuristic to identify patterns.

The results indicated that during senior secondary education, a large majority of the students described a study strategy that was characterised as 'committing to memory and understand', although they hold more or less explicit constructivist believes about knowledge and learning. Another revelation was that at high school level committing to memory and understanding had been sufficient to meet the examination demands. Less than half of the students reported learning strategies theoretically in balance with their regulation strategies and with their conceptions of learning. Another remarkable finding was that internal and external regulation did not seem mutually exclusive. The majority of the medical students at the beginning of the course seemed to be keen to become doctors and was intrinsically motivated. Students expected that things would become more difficult and that more effort would be required at the university. At the same time they expected a shift toward more responsibility and self-direction. Based on their experience in secondary education, students had developed concepts of the ideal student and of ideal teaching and learning that seemed to be related.

Furthermore, the results supported the view that Asian learners combine memorizing and under'standing, as opposed to their Western counterparts. The interview results supported the claim that Asian learners attribute their performances mainly to effort and study skill and that they place high value on academic engagement. Thus, the new entrants had the right frame of mind and chances were that they could adapt to a student oriented learning environment, provided there would be sufficient support during the transition period.

Chapter 6 is about the development of the measuring instrument, Adayayana Rata Prakasha Malawa (ARPM), aimed to assess aspects of learning such as cognitive processing and regulation strategies, learning orientations and conceptions of learning, and about its validation process. The aims of the studies described in Chapter 6 were first to develop a valid and reliable instrument to measure learning patterns of Sri Lankan undergraduate medical students, based on the Inventory of Learning styles (ILS) developed in the Netherlands. Secondly, to research students' reported use of learning activities, their regulation of the learning process, learning orientations and conceptions of learning at the Faculty of Medicine, University of Peradeniya within the existing conventional curriculum.

The ILS was translated into the Sinhala language and the missing learning elements detected during student interviews were added to the appropriate scales. This resulted in the formation of a 123-item questionnaire, the ARPM version 1 that was subsequently pilot tested among a group of new entrants to the faculty of Dental science. The improved version 2 of the ARPM was administered to the new entrants, and medical students in the first, third and fourth years of study at the Faculty of Medicine, University of Peradeniya 
in the year 2000. Besides, through a personal data questionnaire information was gathered about students' age, gender, successful advanced level attempt, aggregate mark, parental education, employment and academic locus of control. Internal consistencies, Cronbach alpha $(\alpha)$, were computed for the 20 different scales of the inventory for the whole group of students, and for each group separately. Means and standard deviations for the ARPM scales were computed for the different groups of students. Principal component analyses with Varimax rotation were executed to examine the relationships between the ARPMvariables for the whole group of students. Furthermore, for each student, factor scores were computed. Relationships between ARPM-variables and personal, contextual, and performance variables were computed using the statistical techniques of analysis of variance, correlation coefficients and independent sample t-tests. Regression analysis was conducted to identify relationships between the academic performance data and students' factor scores.

The results suggest that the Inventory of Learning Styles could be adapted to measure learning patterns among Sri Lankan students. The underlying structures of three of the four factors, namely the meaning directed learning pattern, reproduction directed learning pattern and undirected learning pattern, were similar with those of a Dutch and an Indonesian study that served as comparison studies. Although it was expected to find a clear, distinct application directed learning pattern among the more advanced medical students, such a factor was absent. Instead, a passive idealistic factor with high loadings on all the conceptions was found, similar to the results of two other Asian studies.

Chapter 7 investigated the differences and similarities between the results of the Indonesian study, the Dutch study and the Sri Lankan study, which all used the ILS or one of its adapted versions, i.e. Inventarisasi Cara Belajar (ICB) and the Adyayana Rata Prakasha Malawa (ARPM), in the identification of learning patterns. The aim of this study was to investigate the cross-cultural differences between two Asian and one Western cultures with respect to the reported use of learning strategies, conceptions of learning, learning orientations and learning patterns of first year university students as identified by the respective inventories. The present study was a meta-analysis of the Sri Lankan study and two large-scale studies carried out in the Netherlands and Indonesia. The differences between the inventory scale scores of Sri Lankan and others were investigated using two-tailed t-tests. Learning patterns were compared by contrasting high positive and negative loadings on extracted four factors. A couple of striking differences could be observed. The Dutch students reported more critical processing, memorizing and rehearsing and less concrete processing, self-regulation and lack of regulation than the Asian students, while the differences between the two Asian groups mostly regard learning orientations and learning conceptions. Secondly, both Asian studies did not show a distinct application directed learning pattern. Instead, a theoretically incongruent pattern, characterized by high loadings of all conceptions of learning, was observed in both of the above. Despite the fact that these groups of students were from different disciplines, the two Asian groups showed similar patterns pointing towards the influence of culture in shaping the learning activities.

The aim of the studies reported in Chapter 8 was to explore the development of students' learning strategies, orientations and conceptions, the consistency and variability of these learning aspects within the MBBS course of studies, and students' perceptions of their learning environment, all in a traditional medical school. A cross sectional study and two longitudinal studies were carried out during the $2^{\text {nd }} M B B S$ course and in the $3^{\text {rd }}$ and $4^{\text {th }}$ years of the undergraduate medical course. Variations in learning strategies among the 
three batches of students were measured by analysis of variance in the cross sectional study. In case of significant differences, a Bonferroni post hoc test was performed. In the longitudinal studies changes between the $1^{\text {st }}$ and $2^{\text {nd }}$ administration were compared using paired samples t-tests concerning all variables. Principal component analyses with Varimax rotation were performed on the ARPM scales after each administration (time 1 and time 2) for the two batches to identify the underlying factors, and their structures were compared. Pearson correlation coefficients were calculated between the inventory scales obtained at the two administrations, at individual student level for each batch of students, as a measure of the degree of consistency or variability. Furthermore, the perceptions of two groups of students about the learning environment were assessed by means of the Course Experience Questionnaire. The observed changes were not consistent with the development of a more constructive type of deep level learning during the beginning and the mid part of the MBBS course. If the results are interpreted as an indication of certain features of the learning environment, the increase in lack of regulation and ambivalence and the decrease in personal interest, self-regulation, concrete processing and relating and structuring strategies can be attributed to an overloaded curriculum, emphasis on didactic teaching and the assessment system. However, it also seemed that students were able to adapt and develop their study habits and this has important educational implications.

The final chapler summarizes the research project as a whole and the results of the empirical studies. The educational implications of the outcomes, directions for change and directions for future research complete this chapter. The results of the studies suggest that Sri Lankan medical students are able to adapt and develop their ways of learning, at least to some extent. This has many educational implications, indicating a chance of success for programs aimed at improving the quality of student learning. Finally, based on the results of the project, it is recommended that the curriculum development process at the Faculty of Medicine, University of Peradeniya addresses the issue of content overload and takes necessary action to enhance the use of constructive learning processes and to develop self-directed learning among medical students. A way forward would be to implement desirable changes in the assessment system, as assessments regulate the learning process to a significant extent. Since the teaching style of the teacher also has a considerable impact on the learning pattern of students, it is essential that staff development activities and learning environment modification are carried out simultaneously. Thus, it is proposed that staff training and research in education are given their due place in the reform agenda.

An important direction for future research would be to study the changes in learning behaviour upon instructional changes. Since instructional changes generally take a long time to have a reasonable impact on the learning process, they need to be studied over a longer period of time. In the present study, most of the changes in learning strategies and other learning elements were studied at group level. However, it will also be informative to study changes at subgroup level, e.g. among high, average and low achieving students separately. Also needed are studies on the effect of all kind of instructional measures and variables, derived from theory on activating instruction or $\mathrm{PBL}$, on learning patterns and learning outcomes, Furthermore, the decreasing trend of attributing academic failure to effort that was evident in the cross sectional study also needs to be investigated in a longitudinal design. Finally, an important question is whether the changes that were found are specific for this period of study, or whether they will continue throughout the course. Thus, studies on the learning patterns of a cohort of students from first year to the end of the final year are much needed. 


\section{Samenvatting}

'Leren' is een uiterst gecompliceerd fenomeen. Dit proefschrift is gebaseerd op een conceptueel model waarin gesteld wordt dat het leren beïnvloed wordt door elkaar beinvloedende factoren. Sociaal-cognitieve theorieën benadrukken de rol van factoren die verband houden met individuele eigenschappen, gedrag en de omgeving. Recente theorieën over leren gaan ervan uit dat kennis situatiegebonden is en deels bepaald wordt door de activiteit, context en cultuur waarbinnen zij wordt ontwikkeld en toegepast. Bovendien heeft empirisch onderzoek aangetoond dat kennis verworven door middel van actieve kennisconstructie van betere kwaliteit, toegankelijker, coherenter en beter toepasbaar is dan kennis verkregen door passief leren.

Sommige onderzoekers zijn van mening dat er in traditionele medische curricula weinig aandacht is voor de invloed van de leeromgeving op het leerproces van de studenten. Het is echter bekend dat er ook in een intellectueel homogene groep veel persoonlijkheids- en omgevingsfactoren zijn die individueel gedrag bepalen en dat dit kan leiden tot een sterke heterogeniteit in de studentenpopulatie binnen cenzelfde discipline. Helaas wordt onderwijs vaak gegeven alsof er geen individuele verschillen bestaan. Bij het ontwikkelen van curricula en het aanpassen van onderwijsmethoden om te komen tot betere leerresultaten kunnen docenten profijt hebben van kennis over leerpatronen, stijlen en -benaderingen van de studenten. Een literatuuronderzoek vond geen Aziatische onderzoeken waarin de leerpatronen van medisch studenten diepgaand werden geanalyseerd. Omdat onderzoek hiernaar van groot belang is voor de besluitvorming over onderwijs en verbetering van de onderwijspraktijk in het medisch onderwijs, zijn de leerpatronen van studenten een belangrijk onderzoeksterrein.

Onderzoek van onderwijs word in dit proefschrift gezien als een zorgvuldig systematisch proces waarin het onderwijsproces onderzocht wordt met als doel de resultaten van het onderwijs te verbeteren. Het is dan ook de taak van de onderzoeker om het leren in het hoger onderwijs zorgvuldig te beschrijven en aan te geven hoe onderwijskwaliteit door onderwijs en toetsing bepaald wordt. Tegen deze achtergrond werd het van belang geacht om onderzoek te doen naar leerstrategieën, opvattingen over leren en leeroriëntaties, onderlinge verbanden hiertussen en eventuele effecten van individuele en omgevingsfactoren op aspecten van het leren van Aziatische medisch studenten in een traditioneel medisch curriculum.

De curriculumhervormingen van de laatste tijd richten zich vooral op verbetering van de kwaliteit van het leerproces van de studenten. Algemeen wordt aangenomen dat expertise tot stand komt door ontwikkeling van domeingebonden competenties en kennistheoretische inzichten en een verschuiving in de richting van zelfgestuurd en betekenisgericht leren. Daarom wordt niet alleen gekeken naar wat studenten moeten leren (inhoud) maar ook hoe ze dat het beste kunnen leren. Dit is van groot belang gezien de steeds grotere snelheid waarmee kennis overbodig wordt. Er is op dit moment een wereldwijde trend om medische curricula te hervormen. Onderwerp van discussie in het hoger onderwijs in Sri Lanka is hoe curricula te veranderen en docenten te trainen opdat ze studenten kunnen stimuleren om zelfgestuurd te leren.

Hel onderzoek dat in dit proefschrift beschreven wordt, heeft mede tot doel het verwerven van inzicht in de leerprocessen van medisch studenten in Sri Lanka als basis voor de ontwikkeling van een goed onderbouwd curriculum. De verwachting was dat analyse van verbanden tussen institutionele en contextuele factoren en leerstrategieën en 
leerresuitaten van de studenten bewijsmateriaal kon leveren om academische staf en bestuurders te overtuigen van de zin van veranderingen.

De empirische onderzoeken die in dit proefschrift beschreven worden, betreffen trends in de ontwikkeling van leerstrategieën en zelfgestuurd leren bij Sri Lankaanse medisch studenten in een traditioneel curriculum. Het hoofddoel van het onderzoeksproject was om inzicht te verwerven in aspecten van het leren van Aziatische geneeskundestudenten in een traditioneel curriculum met betrekking tot leerpatronen en de invloed van individuele en omgevingsfactoren. De onderzoeken zijn met name gericht op de volgende onderzoeksvragen:

(1) Welke studiestrategieën, opvattingen over leren, attributiepatronen, waarden, overtuigingen, verwachtingen ten aanzien van de universitaire studie en studiemotivatie worden gevonden bij geneeskundestudenten in Sri Lanka?

(2) Is een aangepaste versie van de Inventaris van Leerstijlen een betrouwbaar en valide diagnostisch instrument?

(3) Welke leerpatronen worden waargenomen bij Sri Lankaanse medisch studenten?

(4) Welke verbanden zijn er tussen de toegepaste leerstrategieën, opvattingen over leren en leeroriëntaties van de studenten enerzijds en persoonlijke en omgevingsfactoren en studieprestaties anderzijds?

(5) Speelt nationale cultuur een belangrijke rol als verklaring van de leerstrategieën van studenten en de onderlinge verbanden tussen verschillende aspecten van het leren?

(6) Welke trends zijn aanwijsbaar in de ontwikkeling van leerstrategieën?

(7) Hoe ervaren de studenten hun huidige leeromgeving?

Daarnaast is gepoogd om meer inzicht te verwerven in het leren van de Aziatische student in het algemeen. Samengevat, zijn de onderzoeken erop gericht een grote hoeveelheid gegevens te verzamelen ter ondersteuning van besluitvorming over onderwijs en docentenprofessionalisering.

Hoofdstuk 1 geeft een algemene inleiding op het project als geheel en beschrijft de hoofddoelstellingen.

Hoofdstuk 2 probeert de lezer inzicht te verschaffen in het hoger-onderwijssysteem in Sri Lanka, met name het medisch onderwijs en de ontwikkelingen tot nu toe. Ook worden de sterke en zwakke punten van de huidige basisartsopleiding in Sri Lanka besproken.

Hoofdstuk 3 beschrijft de theoretische basis van de empirische onderzoeken: recente inzichten over onderwijs en leren; algemeen aanvaarde methoden om aspecten van leren in het hoger onderwijs te meten; bevindingen uit empirisch onderzoek naar de toepassing van leerstrategieën door studenten in het hoger onderwijs in de geneeskunde en andere vakken zowel in westerse als in Aziatische landen; een overzicht van de invloed van cultuur op leergedrag; en innovatieve onderwijsmethoden in de artsopleiding, zoals probleemgestuurd en procesgericht onderwijs.

Hoofdstuk 4 beschrijft het conceptuele model dat de basis vormt van dit proefschrift. Het algemene doel van het project was om bij Srilankaanse geneeskundestudenten de relatie tussen individuele factoren (leeroriëntaties en opvattingen over leren), de leeromgeving, de nationale cultuur en gerapporteerde leerstrategieën te onderzoeken.

Het doel van het kwalitatieve onderzoek dat beschreven wordt in Hoofdstuk 5 was te komen tot verschillende beschrijvende categorieën voor de vier belangrijkste leercomponenten: cognitieve verwerkingsprocessen, regulerende activiteiten, 
studiemotivatie en opvattingen over leren. Daarnaast richtte het onderzoek zich op de relaties tussen de beschreven componenten, de ideeën van aankomende medisch studenten over factoren die hun leren in de laatste jaren op de middelbare school ten goede kwamen, de eigenschappen van de ideale student, de verwachtingen van de studenten ten aanzien van hun studie en de locus of control betreffende de studie. De onderzoeker nam op basis van vrijwilligheid interviews af bij 32 aankomende eerstejaarsstudenten. De transcripties van de semi-gestructureerde interviews werden fenomenologisch geanalyseerd op basis van de hierboven beschreven hoofddomeinen. In het onderzoek naar de opvattingen van de studenten over leren en kennis werd een andere methode toegepast. Als richtlijn voor het analyseren van de ideeën van de studenten over leren diende een schaal beschreven door Lonka, Joram en Bryson (1996) en het attirbutiemodel van Weiner werd gebruikt voor het coderen van fragmenten uit de interviews aangaande aspecten waaraan studenten studiesucces en -mislukking toeschreven. De relatie tussen opvattingen over leren, studiestrategie, reguleringsstrategie, studiemotivatie en verwachtingen ten aanzien van de universitaire studie werden onderzocht met behulp van een tabel bestaande uit eerder ontwikkelde categorieën voor het bepalen van patronen, waarbij voor patroonherkenning gebruik werd gemaakt van bevindingen van Vermunt over integratie van opvattingen over leren, studiemotivatie en studiestrategieën.

De resultaten geven aan dat de studiestrategie van de grote meerderheid van de studenten op de middelbare school gekenschetst kan worden als 'uit het hoofd leren en begrijpen', hoewel hun opvattingen over leren het best gekwalificeerd kunnen worden als meer of minder expliciet constructief. Een andere bevinding is dat op de middelbare school uit het hoofd leren en begrijpen voldoende zijn om examens te halen. De leerstrategieën van minder dan de helft van de studenten passen theoretisch gezien bij hun reguleringsstrategieën en opvattingen over leren. Bij aanvang van de studie tonen de meeste geneeskundestudenten een sterke intrinsieke motivatie om arts te worden. Zij verwachten dat het moeilijker zal worden en dat zij aan de universiteit harder moeten werken. Tegelijkertijd verwachten ze dat ze meer verantwoordelijkheid krijgen en meer zelfgestuurd zullen studeren. Op basis van hun ervaringen op de middelbare school hebben de studenten een samenhangend beeld gevormd van de ideale student en ideaal onderwijs en studeren.

De resultaten bevestigen de opvatting dat Aziatische studenten in tegenstelling tot hun westerse collega's uit het hoofd leren en begrijpen combineren. De resultaten van de interviews ondersteunen de opvatting dat Aziatische studenten hun prestaties vooral toeschrijven aan eigen inspanningen en studievaardigheid en dat zij veel waarde hechten aan betrokkenheid bij de studie. Dit geeft an dat de aankomende studenten een goede studiehouding hebben en een goede kans om zich aan te passen aan een leeromgeving waarin de student centraal staat, mits zij bij deze overgang voldoende begeleid worden.

Hoofdstuk 6 behandelt de ontwikkeling van de Adayayana Rata Prakahs Malawa (ARPM), een meetinstrument voor aspecten van leren zoals kennisverwerking en reguleringsstrategieën, leeroriëntaties en opvattingen over leren, en de validering van dit instrument. Het doel van de in dit hoofdstuk beschreven onderzoeken was in de eerste plaats om op basis van de in Nederland ontwikkelde Inventaris van Leerstijlen (ILS) een valide en betrouwbaar meetinstrument te ontwikkelen voor de leerpatronen van medisch studenten in Sri Lanka. In de tweede plaats was het onderzoek gericht op de door studenten gerapporteerde leeractiviteiten, regulering van leerprocessen, leeroriëntaties en 
opvattingen over leren. Dit onderzoek werd uitgevoerd bij studenten die het traditionele curriculum aan de medische faculteit van de Universiteit van Peradeniya volgden.

De ILS werd in het Sinhala vertaald en tijdens de interviews gesignaleerde ontbrekende leeraspecten werden aan de daartoe geëigende schalen toegevoegd. Dit resulteerde in een vragenlijst van 123 vragen, de ARPM versie 1, die getest werd bij een groep eerstejaarsstudenten van de faculteit tandheelkunde. De verbeterde tweede versie van de ARPM werd in 2000 afgenomen bij aankomende studenten, en bij eerste-, derdeen vierdejaars studenten aan de geneeskundefaculteit van Peradeniya. Daarnaast werden door middel van een vragenlijst persoonlijke gegevens verzameld over leeftijd, geslacht, deelname aan en slagen voor het examen op hoger niveau, gemiddeld cijfer, onderwijsniveau van de ouders, werk en locus of control ten aanzien van de studie. Voor de 20 schalen van de lijst werd de interne consistentie, Cronbachs alfa, berekend voor de groep studenten als geheel en voor de verschillende groepen afzonderlijk. Gemiddelde score en standaarddeviatie op de ARPM-schalen werd berekend voor de verschillende groepen studenten. Met behulp van principale-componentenanalyse werden de relaties tussen de ARPM-variabelen bepaald voor de groep studenten als geheel. Daarnaast werden factorscores per student berekend. De relaties tussen de ARPM-variabelen en individuele, contextgebonden en studieprestatiegebonden variabelen werden berekend met behulp van variantieanalyse, correlatiecoëfficiënten en twee-steekproeven t-toetsen. Met behulp van regressieanalyse werd de relatie tussen de gegevens over studieprestaties en de factorscores van de studenten bepaald.

Uit de resultaten kan worden afgeleid dat de aangepaste versie van de ILS geschikt is om de leerpatronen van Sri Lankaanse studenten te meten. De onderliggende structuur van drie van de vier factoren, namelijk het betekenisgerichte leerpatroon, hel reproductiegerichte leerpatroon en het ongerichte leerpatroon, is in overeenstemming met de resultaten van een Nederlands en een Indonesische onderzoek die als vergelijkingsmateriaal fungeerden. Hoewel een uitgesproken toepassingsgericht leerpatroon werd verwacht bij de meer gevorderde studenten werd een dergelijke factor niet gevonden. In plaats daarvan werd een passieve, idealistische factor met hoge ladingen op alle concepten gevonden, hetgeen de resultaten van twee andere Aziatische onderzoeken bevestigt.

In Hoofdstuk 7 wordt een onderzoek gepresenteerd naar de verschillen en overeenkomsten tussen een Indonesisch, Nederlandse en Sri Lankaans onderzoek waarin leerpatronen bepaald werden met behulp van de ILS of een aangepaste versie daarvan: de Inventarisasi Cara Belajar (ICB) en de Adyayana Rata Prakasha Malawa (ARPM). Het doel van dit onderzoek was om interculturele verschillen te onderzoeken tussen twee Aziatische en een westerse cultuur ten aanzien van gerapporteerde toepassing van leerstrategieën, opvattingen over leren, leeroriëntaties en leerpatronen van eerstejaarsstudenten zoals gemeten met de verschillende vragenlijsten. Het onderzoek behelsde een meta-analyse van het Srilankaanse onderzoek en twee grootschalige onderzoeken in Nederland en Indonesië. Met behulp van tweezijdige t-toetsen werden de verschillen tussen de ILS-scores uit de verschillende onderzoeken getoetst. Om de verschillen tussen leerpatronen te onderzoeken werden hoge positieve en negatieve ladingen op de vier gevonden factoren met elkaar vergeleken. Er werden enkele opvallende verschillen gevonden. Vergeleken met de Aziatische studenten rapporteren de Nederlandse studenten meer kritische verwerking, leren en herhalen, en minder concrete verwerking, zelfregulering en gebrek aan regulering. De verschillen tussen de Aziatische groepen betreffen hoofdzakelijk leeroriëntaties en opvattingen over leren. In beide 
Aziatische onderzoeken is geen uitgesproken toepassingsgericht leerpatroon gevonden. In plaats daarvan werd in deze onderzoeken een patroon gevonden dat theoretisch niet consistent is en waarin hoge ladingen gevonden worden op alle opvattingen over leren. Hoewel de studenten in deze onderzoeken uit verschillende disciplines afkomstig zijn, geven beide Aziatische groepen vergelijkbare patronen te zien. Dit wijst op een mogelijke invloed van cultuur op de vorming van leeractiviteiten.

De onderzoeken die in Hoofdstuk 8 beschreven worden, zijn uitgevoerd bij studenten aan een traditionele medische opleiding en richtten zich op de ontwikkeling van leerstrategieën, -oriëntaties en opvattingen over leren, de consistentie en verschillen in deze leeraspecten tijdens de basisartsopleiding en de percepties van de studenten aangaande hun leeromgeving. Het onderzoek bestond uit een transversaal onderzoek en twee longitudinale onderzoeken in jaar 1 en 2 , jaar 3 en jaar 4 van de basisartsopleiding. In het transversale onderzoek werden verschillen in leerstrategieën tussen de drie groepen studenten bepaald door middel van variantieanalyse. Bij significante verschillen werd een Bonferroni post-hoc-toets uitgevoerd. In de longitudinale onderzoeken werden met behulp van gepaarde t-toetsen de verschillen tussen de eerste en tweede afnames vergeleken voor alle variabelen. $\mathrm{Na}$ elke afname (T1 en $\mathrm{T} 2$ ) werden principale componentenanalyses met varimaxrotatie uitgevoerd op de ARPM-schalen en werd de schaalstructuur vergeleken. Om de mate van consistentie of variatie te bepalen werden Pearson correlatiecoëfficiënten berekend voor de schalen van beide afnames, zowel op het niveau van de individuele student als voor elke groep studenten. Daarnaast werden de percepties van beide groepen studenten ten aanzien van de leeromgeving gemeten met behulp van de "Course Experience Questionnaire". De veranderingen die gevonden werden aan het begin en halverwege het curriculum zijn niet in overeenstemming met een ontwikkeling in de richting van een vorm van constructief diep leren. Als de resultaten gezien worden als een aanwijzing voor bepaalde kenmerken van de leeromgeving kan de toename in gebrek aan regulatie en ambivalentie en de teruggang in individuele belangstelling, zelfregulering, concrete verwerking en verbindende en structurerende strategieën toegeschreven worden aan een overladen curriculum, nadruk op docentgericht leren en het toetssysteem. Maar studenten lijken ook in staat hun leergewoonten aan te passen en te ontwikkelen en dit heeft belangrijke implicaties voor het onderwijs.

Het laatste hoofdstuk geeft een samenvatting van het onderzoeksproject als geheel en de resultaten van het empirisch onderzoek. Ook komen de implicaties voor het onderwijs en suggesties voor veranderingen en verder onderzoek aan de orde. De resultaten geven aan dat medisch studenten in Sri Lanka, in ieder geval tot op zekere hoogte, in staat zijn hun leerstrategieën aan te passen en te ontwikkelen. Dit heeft belangrijke consequenties voor het onderwijs en daarnaast wijzen de resultaten erop dat programma's om de kwaliteit van het leren van studenten te verbeteren een goede kans van slagen hebben. Ten slotte, wordt op basis van de resultaten van het project de aanbeveling gedaan om bij curriculumvernieuwing aan de medische faculteit van de Universiteit van Peradeniya aandacht te besteden aan de inhoud van het overladen curriculum, het bevorderen van constructieve leerprocessen en zelfgestuurd leren bij studenten. Een goede manier om dit te realiseren zijn veranderingen in het toetssysteem, omdat toetsing het leerproces in hoge mate bepaalt. Omdat doceerstijl ook belangrijk is voor het leerpatroon van de student dienen docentenprofessionalisering en aanpassing van de leeromgeving tegelijkertijd te worden aangepakt. Er wordt dan ook voorgesteld om docententraining en onderzoek van onderwijs een plaats te geven in het vernieuwingsprogramma. 
Een belangrijk thema voor toekomstig onderzoek zijn veranderingen in leergedrag na invoering van onderwijsveranderingen. Omdat het meestal lang duurt voordat de resultaten van dergelijke veranderingen merkbaar worden, is longitudinaal onderzoek nodig. De onderzoeken in dit proefschrift naar veranderingen in leerstrategieën en andere aspecten van het leren zijn meestal op groepsniveau uitgevoerd. Het zou echter zinvol zijn om veranderingen te onderzoeken op het niveau van subgroepen, bijvoorbeeld bij studenten met goede, gemiddelde en slechte resultaten. Ook moet onderzoek gedaan worden op basis van de theorie over activerend leren of probleemgestuurd leren naar de effecten van allerlei onderwijskundige maatregelen en variabelen op leerpatronen en uitkomsten. Daarnaast dient longitudinaal onderzoek gedaan te worden naar de in het transversale onderzoek gevonden afnemende tendens om studiefalen toe te schrijven aan inspanning. Een belangrijke vraag is ten slotte of de gevonden veranderingen specifiek zijn voor de studieperiode of dat ze zich in de loop van het curriculum zullen consolideren en voortzetten. Daarom is het belangrijk dat onderzoek gedaan wordt naar de leerpatronen van studentencohorten van het eerste tot en met het laatste jaar. 
ppendix

\section{Ippendix}

\section{- $\quad$ TTTRIBUTES OF AN IDEAL STUDENT}

\section{1.a BROAD KNOWLEDGE BASE}

1.a.1 (s2) "Somebody with a broad knowledge base, knows correct facts", 1.a.lb (s6) "... A student who knows the subject to a great depth."

1.b USE OF EFFECTIVE STUDY STRATEGIES

1.b.1a Processing
1.b.1a.1 (s15)
"... apply knowledge practically"
1.b.1a.2 (s13 \& 24) "does not believe things just because somebody tells that to him but uses his knowledge and ac- cepts what is being told"

1.b.1b Self and Resource management
1.b.1b.1 (s19)
"plans how to study to achieve the set target".
1.b.1b.2 (s24)
"does not postpone studies for other reasons,
1.b.1b.3 (s11) sets personal goals", "manages time efficiently, completes work on schedule".

\section{1.c INTRISIC MOTIVATION}

1.c.1 (s13) "very inquisitive"

1.c.2 (s19) "interested in studies, likes to study further",

\section{1.d ATTITUDES AND PERSONALITY}

1.d.1a Social Outgoing, responsible, cooperative, respect others

1.d.1a.1 (s21)

1.d.1a.2(s2)

1.d.1a.3 (s15)

1.d.1a.4 (s4)
"... somebody who devoles his/her time for both academic and extracurricular activities"

".... responsible"

"... not a book worm"

"... who can work with others"

1.d.1b Commitment to Studies

1.d.1b.1 (s19)

"committed, gives highest priority for studies,

1.d.1b.2(s7) strives to achieve the set goals"

"... a keen student" 
Appendix

1.d.Ic Caring and Helping

1.d.1c.1 (s28) " "... helpful to others"

1.d.1c.2(s25) "person who is not selfish"

\section{FACTORS FACILITATING I.EARNING}

2.a GOOD LEARNING

2.a.la Active engagement in learning

2.a.1a.1 (s6) "Tuition classes. The teacher taught theory first. We were asked to write answers to particular questions. Thus, application of knowledge in the form of problem solving helped learning."

2.a.1a.2(s24)

"The physics teacher taught some theory and then she gave us a problem to solve. When we have tried to solve that on our own she works that out on the board so that we can lind out whether we have followed the correct steps. When I work out on my own I understand better."

2.a.Ib Cooperative learning

2.a.lb.1 (s5)

"Discussions with friends on difficult subject aleas. There was sharing of views, and was interesting. Every body were active and therefore, more altentive."

2.a.1c Self directed learning

2.a.1c.1 (s7)

"Study on my own. I lirst check whether I can answer questions in relation to a particular subject area. If I cannol answer, then I will refer my own books or actively search for that information and try to fill the gaps in knowledge. I feel I learn better that way."

2.a.1d Practical

2.a.1d.1 (s19)

2.a.1d.2(s3)

"The Botany lesson. In this lesson we were taken to the school garden and were allowed to make own obscrvations and collect parts of trees. Wc learn much more than just reading from a book. We were in a very relaxed mood and were actively involved in exploring." "Zoology - dissection of Thilapia. Where I had to dissect the fish on my own. I learned the characteristics of that fish from this exercise 
rather than just listening to the teacher"s description alone."

"I like practical classes. Can understand subject matter very easily when we actually sec with the naked eye. Example the vertebrae when given to us we touched, observed and learned about the articular surfaces much easily."

TEACHING

Giving appropriate feedback

2.b.la.1 (s11) "When I make mistakes in writing answers 10 questions. I get the teachers to correct the answers that I have written. Then I become aware of my mistakes following that I go home and refer my notes. Since I was made aware of the mistakes, I correct them and that registers in my mind."

Making complex things easier to understand

2.b.1b.1 (s1) "Chemistry lessons. That teacher starts from simple and easy to understand things that we already knew and then goes on to complicated subject matter.

2.b.1b.2(s29) "Zoology lesson" the teacher explained in a very simple way using diagrams and a lot of examples."

Help construct knowledge

2.b.1c.1 (s14)

"Chemistry tuition lessons. The teaching style of the teacher where he first explained the topic, then asked questions from us, and taught the subject by answering questions. When the students gave an answer, he modified that and the lesson was done in a question and answer form. This particular teaching style drew our attention throughout the lecture. Use of mnemonics helped us to remember the subject matter." This teacher has simplified the subject matter and helped to construct meaning.

I Motivation

2.b.1d.1 (s2) "Tuition lessons. Tuition classes motivated me to learn. The teaching method of the teacher was precise. Tuition lectures were complete... covering all aspects. Tuition master used different au- 
dio-visual aids. His habit of summarizing in the middle of lecture was particularly useful. All this motivated me to learn." In the case of this student the teacher has made it easy to construct the meaning and that has aroused an interest in the subject.

2.b.1d.2 (s1) "Chemistry teacher always made sure to encourage us to learn. He even taught complicated areas in simple terms ... He gave no prior indication of the complexity of the subject. His teaching style and the sense of humour aroused our interest in the subject"

2.b.1d.3 (23) $\quad \cdots$.. I was able to concentrate the whole lesson. The teacher gave us the impression that if I can understand the lesson that it will help me to understand other subjects. He got us to think during the lesson. He boosted my moral and I developed an interest in the subject and studied hard."

\section{STUDY STRATEGILS}

\section{3.a COGNITIVE PROCESSING STRATEGIES}

3.a.1a Simply memorizing facts
3.a.1a.1 (s22)
"I did memorize as it is a requirement to pass the Advanced level examination (it is unfortunate that the exam checks the ability to recall, ...this
3.a.la.2(s16) approach was found to be boring)". "I read the notes several times. Memorized some of the simple facts".

3.a.1b Commit to memory and understand

3.a.1b.1 (s11)

"First I take all the notes I have under a particular heading. Then I read, I try to close the notes and recall what I have read. If I cannot recall something I go back and study that. I repeat the process until 1 understand and remember that part".

3.a.1b.2 (s19) "I first read the note. Then I closed the book and tried to recapitulate what I have read that way it helped me to understand."

3.a.lc Studying the subject matter step by step

3.a.1c.1 (s11) "I study one section and alter completing that section I move on to the next. For example I take the urinary system I study the structure and 
function of this system answer the questions on that and move on to the next section".

3.a.Id Actively relating and structuring of information

3.a.1d.1 (s23)

"While I was studying I made comparisons between the different parts of subject matter. I attempted to relate new information to what I have studied".

3.a.le Applying what is learned

3.a.le.1 (s4) "I started writing answers to questions, then solve problems in chemistry, I referred the notes during this process and that helped me to understand."

\section{3.b REGULATION STRATEGIES}

3.b.la Extcmal regulation of lcarning processes and results

\section{3.b.1a.1 The tuition class teacher}

3.b.1a.1.1 (s8) "I attended tuition classes for all four subjects. I select the tuition teachers who could explain well and attend their classes. For physics I attended two classes."

3.b.1a.1.2 (s19) "Entirely dependent on teachers notes. I did not read text books although they were available at home."

3.b.1a.2 Past paper questions.

3.b.1a.2.1 (s10)

"Write answers to past paper questions after studying or during studying that indicated how much I knew and whether I was able to understand what is expected. I collected the past papers of the last ten years and wrote answers to those queslions. Some of the questions are being repeated therefore this strategy helped me to succeed".

\section{3.b.1a.3 Monitor advancement through past papers questions}

3.b.1a.3.1 (s30) "I analyzed my term test marks. If I could score high marks I thought I was progressing well". 
3.b.1a.3.2(s17)

3.b. 1a.3.3 (s18)
"I do past questions and get leedback from teachers as to what I have done suflicient."

"The past paper questions and their model answers directed me in selecting what to study."

3.b.1b Self-regulation of learning content and process

3.b.1b.1 Monitoring study progress

3.b.1b.1.1 (s28) "I studied alone. Alter studying a particular arca. I checked my progress by answering either past paper questions or I make my own questions and try to answer those".

3.b.lb.1.2(s5) "If I can make a complete note after studying a particular area $I$ have understood"

3.b.1b.2 Plaming the study process, effort and organization:

3.b.1b.2.1 (s28) "I read about effeclive study methods such as SQ3R and practiced those in my studies. I concentrated for a limited period at a stretch and had breaks in betwecn studies, I also followed a time table".

3.b.1b.2.2( $(\$ 25) \quad$ "I was determined to complete that day"s work on that day itself".

3.b.1b.3 Self-selecting the learning activities to deepen understanding

3.b.16.3.1 Reading textbooks or articles

3.b.1b.3.1.1 (s2.3) "Sometimes during studies we read text books and articles to broaden our knowledge".

3.b.1b.3.1.2(s12) "When the lesson is not clear I read relevant text books"

3.b.1b.3.2 Making their own short notes or charts and diagrams, connecting the relevant areas

3.b.1d.1.2.1 (s21) "I first read the notes \& books and then thought about what I have studied. on my own. Later I make my notes, diagrams, graphs or flow charts. Used a lot of colours to highlight facts". 
Appendix

3.b.1b.3.3 Asking for clarification of problematic areas with teachers or students

3.b.1b.3.3.1 (s32) "I go to the teachers and get things clarified outside teaching hours."

\section{3.c COOPERATIVE LEARNING}

3.c.1 (S9): "We wrote answers to questions separately and discussed our answers sometimes taught each other so that what was not clear to me was explained by another".

3.c.2 (s20): "After answering questions from a particular area the friends got together and discussed and argued over the answers"

\section{5. $\quad$ ATTRIBUTIONS}

\section{5.a ATTRIBUTIONS OF SUCCESS}

5.a.1 (s17) "I worked hard much more than the first attempt. This time I concentrated on past paper questions that helped me because some of the questions were repeated." Implicit in this statement is the fact that this student has not faired well in the previous attempt as has not worked hard and has been successful this time due to hard work and some luck.

5.a.2. (s3) "I always thought that determination and hard work resulted in me getting a high aggregate at the university selection examination".

5.a.3. (s15) "I was very happy. I think I worked hard, devoted much more time for studies in my second attempt. I feel one could get a high aggregate if he has worked hard."

5.a.4. (s14) "I was very happy. I think this is the result of studying hard".

\section{5.b ATTRIBUTIONS OF FAILURE}

5.b.1 (s23) "I thought I have to change my study pattern and I must practice answering questions". In this quote the internal attribution is implicit.

5.b.2 (s24) "After the exam I feel that if I had studied hard. I could have scored very much better, especially in the first attempt. I must say I was lazy. I did not work to the best of my ability. I did not devote much time for studies."

5.b.3 (s15) "I must say that in the first attempt I did not spend sufficient time to study. That was the reason why I could not get a high score. There were some questions, which I thought I could have answered if I have studied. Since I have not studied I knew I could not write a successful answer". 
5.b.4 (s6) "This happened during the end of term examination. I thought I have not studied cnough. I met the school teachers to find out my deficiencies and decided to study harder".

\section{STUDENT EXPECTATIONS ABOUT LEARNING IN HIGHER CDUCATION}

6.a. GREATER EFFORT

6.a.1a.1 (s19) "I will have to work hard. According to my sister ... nobody will try to help from outside. We will have to do most of the work."

6.a.1a.2 (s26) "I think we will have to work hard. Teachers will help us, but we will have to do a lot of sellstudy. I know this is difficult at the beginning but I feel that this method is more effective".

\section{6.b. DIFFERENCES WITH SCHOOL}

6.b.1a. Learning materials in the University

6.b.1a.1 (s32) "It is essential that we read and gather information since it is useful to have an in depth knowledge"

6.b.1a.2 (s29) "In higher education we will be made to rely less on teachers notes but will have to refer books".

6.b.1a.3 (s25) "We will have to refer books and find out relevant facts unlike Advanced level years where most of the teaching was done by the teachers and we hardly read books. We depended heavily on the teacher"s notes (school or tuition class) to pass the exam".

6.b.la.4 (s22) "I do not think that university teachers will provide lecture notes to us. We will have to do a lot of independent study. We will have to refer books. I do not mind referring books but when we have to write our own notes it is not going to be easy. We will have to spend a lot of time and effort to make our own notes. So it is not like the school days where the tuition teachers provide a set of notes; now onwards we will have to take the initiative."

6.b.1b Student is responsible

6.b.1b.1 (s23)

"I would say while in school teachers did $90 \%$ of the work; they sort of dragged us in. But now we are matured enough to lake responsibility. Student has to do a lot of planning and think and work on my own to succeed". 
Appendix

6.b.1b.2 (s10) " "... everything was given to us by the teachers. In the university the student has to be entirely responsible for his/her learning. I think it is going to be interesting! Teachers will not provide cverything like in school ... they will only guide or direct us. The other thing in school teacher are blamed for not covering the syllabus and so on, in higher education this kind of excuses are not valid".

6.b.1b.3 (s3) "It is going to be very different to school. Schoolteacher gives notes, diagrams, and everything to us. In the university the student has to be entirely responsible for his/her learning. I think it is going to be interesting".

6.b.1b.4 (s4) "Unlike in school the responsibility of learning is shifted towards student. During Advanced level days student plays a small part it is the teacher who drives the student".

\section{6.c PRACTICAL APPLICATION OF KNOWLEDGE AND APPLICATION OF SKILLS}

6.c.1 (s9) "It is a training to do a job later. Therefore we must practice, unlike in $A$ dvanced level years where we had to memorize subject matter only to pass the examination. Undergraduate course in medicine is job oriented, therefore understanding is important. It should not be a case of memorizing facts to pass exams".

6.c.2 (s6) "We must attempt to understand the logic behind what is taught, practical applications of knowledge gained and get rid of memorizing lacts for its own sake".

\section{6.d. FROM RECEPTION TO DISCUSSION}

6.d.1 (s20) "Share our thoughts, take part in discussions, in fact, cooperative learning will be beneficial, specially when we have to gain a lot of knowledge*.

6.d.2 (s19) "It is advantageous to have a good discussion group."

6.d.3 (s2) "We will have a lot of opportunities to discuss with students and teachers".

\section{6.e. SIMILAR TO SCHOOL}

6.e.1 (s22) "During the short stay in the faculty I have found it is very competitive like in school. Contrary to the common belief about university education it is subject oriented ... I feel it is not all that different from school! A narrow approach is being taken. Certain additional skills are not considered in the training". 
Appendix

\section{STUDIENT EXPECTATIONS OF THE ROLE OF A UNIVERSITY TEACHER}

\section{7.a ENABLE SELF-REGULATION}

7.a.1 (s9) $\quad$ *... I think the teachers will direct us in the correct path. They will give us topics and subtopics that we need to learn. We will have to refer books and learn. We have to think broadly."

7.a.2 (s31) "Teachers will clarify the objectives ... so that we can work independently."

7.a.3 (s1) "Unlike the teachers in schools who force us to study and then dictate notes and shoulder greater part of the responsibility of learning, we must be instructed to do certain things, refer books, may be discussions encourage us asking questions for clarification purposes and make us think and work independently..."

\section{7.b SUPPORT LEARNING}

7.b.1 (s3) *... point out weaknesses of students, provide constructive feedback and keep a close cye on the activities of students"

7.b.2 (s8) "Teacher should commence a dialogue with the students on their learning problems, provide constructive feedback to students. Students should get the feeling that they are being supervised."

7.b.3 (s1) "Unlike the teachers in schools it is expected that there will be a good relationship, ensure equality they will encourage discussions and interactive learning and be approachable, friendly and be flexible, respect views of others."

7.b.4 (s6) "Point out the weaknesses of students and provide constructive feedback".

\section{7.c FUNCTION AS A TEACHER}

7.c.1 (s23) "...guide the students without confusing us. Give simple and clear instructions. Lectures to be well organized in a logical manner so that we can understand easily".

7.c.2 (s7) "Teacher must cover the syllabus".

\section{STUDY MOTIVES}

\section{8.a EXTRINSIC MOTIVES}

8.a.1a Extrinsic with a strong emotional component

8.a.la.1 (s6) "I was not ambitious. I entered the medical faculty because I got a high aggregate (...). But my teachers continuously encouraged me to do medicine." 
Appendix

8.a. 1 b Extrinsic job related

8.a.Ib.1 (s18) "When I was beginning the A/L course the social status of a doctor was very high that also influenced my decision. Since we belong to the middle class... I thought that if I can work hard and become a doctor I could lead a comfortable life".

8.a.1b.2 (s22) "I had this liking from my childhood. I had a good attitude about doctors. Well, the social status of a doctor inlluenced my decision. People look up to doctors. As a doctor we can earn money and when I was deciding to do Bioscience it was so that there was a job at the completion of training. The situation is not going to be that easy later. I do not know much about the medical training or the course bul the general feeling was that this is the best and especially when I got a high aggregate... I thought I must

8.b. INTRINSIC MOTIVES do medicine and nothing else."

8.b. Ia Intrinsic- strong emotional component

8.b.1a.1 (s1) "From my childhood I had this ambition. Even when parents asked me what I would become in the future. I used to answer "a doctor."

8.b.la.2 (s10) I wanted to become a doctor from my childhood. During my childhood I was getting ill frequently ... I thought I should become a doctor one day. I also thought I am capable "

8.b.la.3 (s17) "I like this job. It is a childhood dream. I was detcrmined to do medicine."

8.b.1a.4 (s9) "From my childhood I had this liking. I wanted to be a doctor."

8.b.1b Intrinsic - job or study related

8.b.1b.1 (s21)

8.b.1b.2 (s28)
"I want to be a physician like..., this doctor was very kind to my grand mother"

"It is my choice. I think a doctor renders a great service. A doctor can save a person with his expertise. I think that is the most rewarding job. Doctors also have more opportunities to serve the public. I am coming from a remote area and I am aware of the hardships faced by our community. I actually want to serve those people who are helpless. I am not very concerned about the salary that I will get but I honestly want to render a service to the people. I have seen the 
way some doctors treat their patients I do not want to follow them but to be kind and fulfill my duties to the best of my ability. I selected to pulsue a carrier in medicine mainly to serve the sick people and also to uplift their lives."

8.b.1b.3 (s4) "I am interested in forensic medicine and detective work."

8.b.1b.4 (s23) "Actually after the O/L results I first followed arts subjects in school, then I did not like that so I shifted to a commerce class. I could not devclop a liking for that area too. I did $\mathrm{O} / \mathrm{L}$ again and then I got better results and I changed my mind to do bioscience. ... So I entered a school at Kurunegala and worked as much as possible and got selected to do medicine. I liked bioscience subjects." 


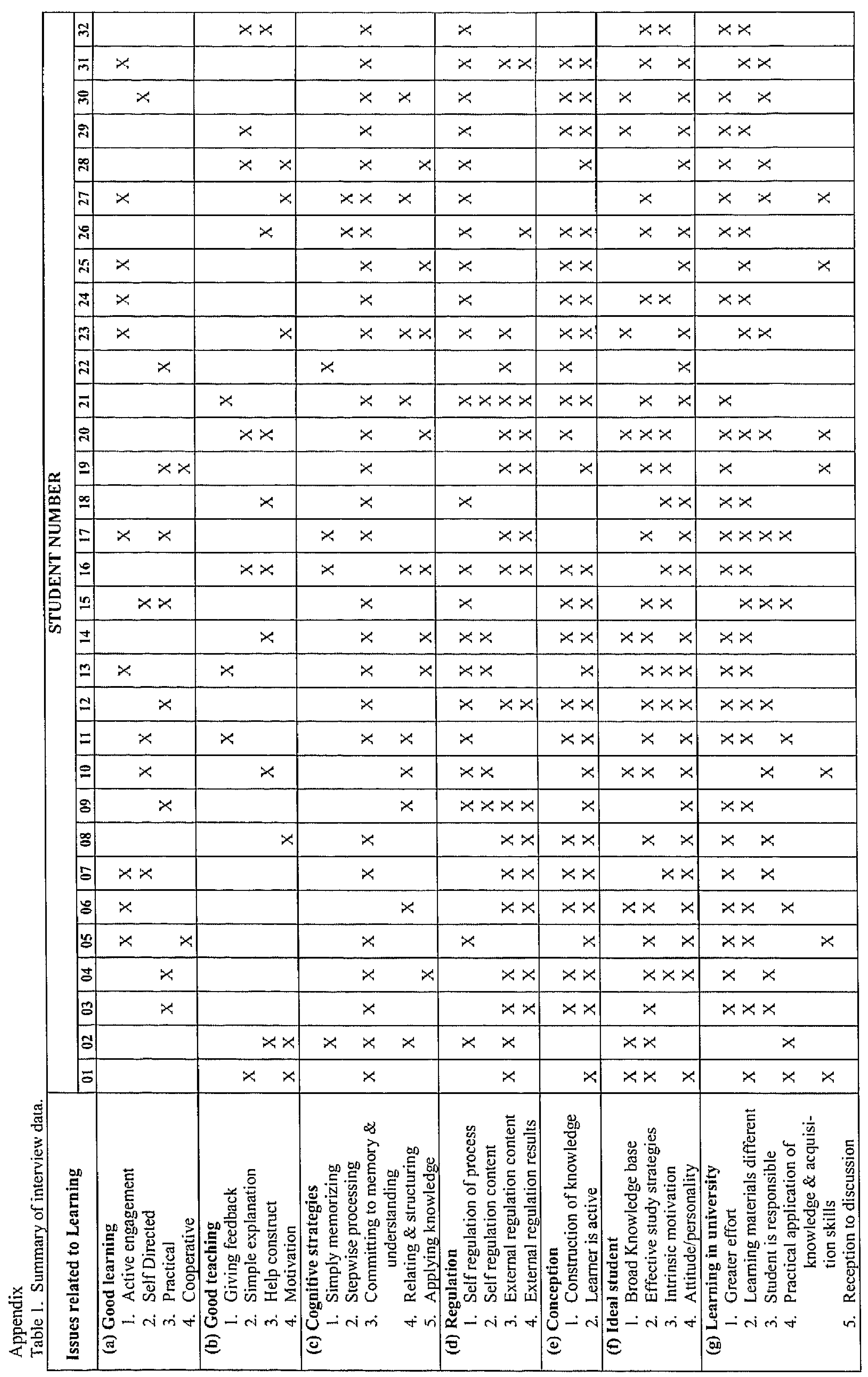




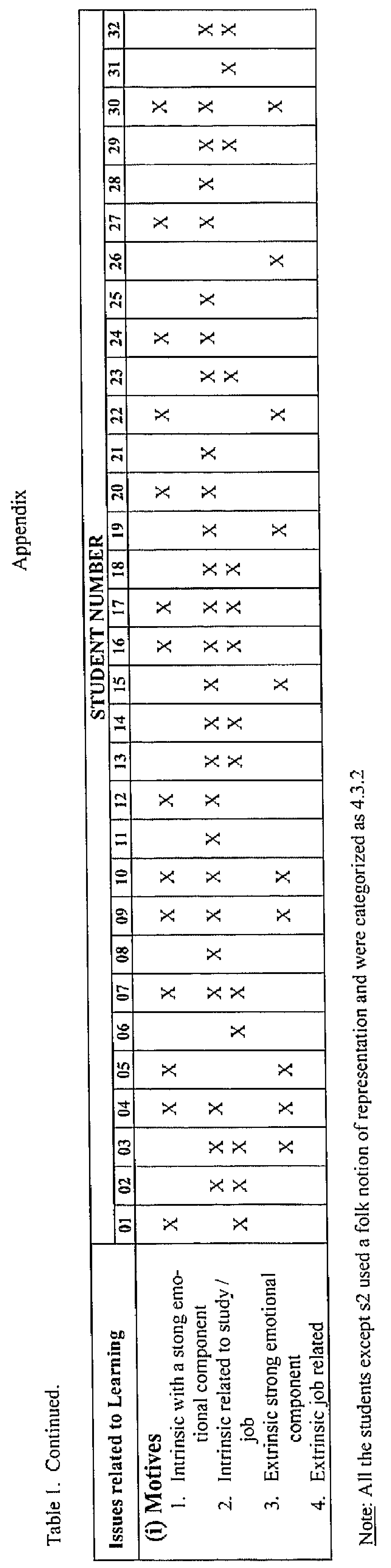

